\title{
Synthesis of Carbamoyl Fluorides via a Selective Fluorinative Beckmann Fragmentation
}

\author{
Jin Woo Song ${ }^{\mathrm{b}, \mathrm{c}}$ and Hee Nam Lim ${ }^{\mathrm{a}, \mathrm{b}^{*}}$ \\ ${ }^{a}$ Department of Chemistry and Biochemistry, Yeungnam University, 280 Daehak-Ro, Gyeongsan, \\ Gyeongbuk, 38541, Republic of Korea \\ ${ }^{b}$ Eco-Friendly New Materials Research Center, Therapeutics\&Biotechnology Division, Research \\ Institute of Chemical Technology (KRICT), 141 Gajeong-ro, Yuseong-gu, Daejeon 34114, Republic of \\ Korea \\ ${ }^{c}$ Department of Chemistry, Korea University, 145 Anam-ro, Seongbuk-gu, Seoul 02841, Republic of \\ Korea
}

1. Materials and Methods

2. Experimental Procedures and Characterization Data

1) Compounds 3a-l, 5a-c.

2) Compounds 7a-l, 4a-l S10-15

3) Compounds 6a-c, 8a-j, 9-10, 14a-c, 15, 16

3. ${ }^{1} \mathrm{H}-\mathrm{NMR},{ }^{13} \mathrm{C}-\mathrm{NMR},{ }^{19} \mathrm{~F}-\mathrm{NMR}$

S21-89 


\section{Materials and Methods}

Dichloromethane $\left(\mathrm{CH}_{2} \mathrm{Cl}_{2}\right)$, Tetrahydrofuran (THF), and Dimethylformamide (DMF) were directly used from SPS system (Hansen, Puresolve MD) without further purification. Thin layer chromatography (TLC) analysis was run on silica gel plates. Most of spots were visualized by exposure to ultraviolet (UV) light ( $254 \mathrm{~nm}$ ). Some spots that were invisible to ultraviolet (UV) used $\mathrm{KMnO}_{4}$ stain solution. High Resolution Mass Spectrometric analysis were performed on JEOL JMS-700 [Ionization mode: EI and FAB; Mass analyser type: Double-focusing type (magnetic sector-electrostatic sector)]. High-resolution mass spectra were reported for the molecular ion $[\mathrm{M}]^{+},[\mathrm{M}+\mathrm{H}]^{+}$. Nuclear magnetic resonance (NMR) spectra were recorded either on Bruker 500 UltraShield, Ascend 400, 300 UltraShield (either on $300,400,500 \mathrm{MHz}$ for ${ }^{1} \mathrm{H}, 376 \mathrm{MHz}$ for ${ }^{19} \mathrm{~F}$ NMR, and either on 100 or $125 \mathrm{MHz}$ for ${ }^{13} \mathrm{C}$ ). The fluorine spectra are unreferenced. Chemical shifts for proton NMR spectra are reported in parts per million (ppm) relative to the singlet at $7.26 \mathrm{ppm}$ for chloroform- $d$, to the quintet at $3.31 \mathrm{ppm}$ for methanol- $d_{4}$, and to the quintet at $2.50 \mathrm{ppm}$ for dimethylsulfoxide- $d_{6}$ and quintet at $2.05 \mathrm{ppm}$ for acetone- $d_{6}$. Chemical shifts for carbon NMR spectra are reported in $77.2 \mathrm{ppm}$ with the center line of triplet for chloroform- $d$, in $49.1 \mathrm{ppm}$ with the center line of the septet for methanol- $d_{4}$ and in $39.5 \mathrm{ppm}$ with the center line of the septet for dimethylsulfoxide- $d_{6}$. Data for ${ }^{1} \mathrm{H}$ NMR were presented as following: chemical shifts $(\delta, \mathrm{ppm})$, multiplicity $(\mathrm{br}=$ broad, $\mathrm{s}=$ singlet, $\mathrm{d}=$ doublet, $\mathrm{t}=$ triplet, $\mathrm{q}=$ quartet, $\mathrm{p}=$ pentet, $\mathrm{dd}=$ doublet of doublets, $\mathrm{m}=$ multiplet $)$, coupling constant $(\mathrm{Hz})$, and integration. The chemical shifts of peaks found were reported for ${ }^{13} \mathrm{C}$ NMR spectra. Infrared spectra were recorded with a Smith Detection ATR-FTIR.

\section{Experimental Procedures and Characterization Data}

The known starting materials including $\mathbf{7} \mathbf{a}^{1}, \mathbf{7} \mathbf{b}^{2}, \mathbf{7} \mathbf{d}^{2}$, and $\mathbf{7} \mathbf{e}^{1}$ were not characterized.

The side-product identified during optimization (Table 1, entry 8):

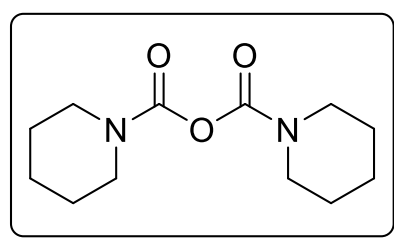

240.1463 . piperidine-1-carboxylic anhydride: light yellow solid; $\mathrm{R}_{f}=0.4$ $(\mathrm{Hex}: \mathrm{EtOAc}=3: 1) ;{ }^{1} \mathrm{H} \mathrm{NMR}\left(400 \mathrm{MHz}, \mathrm{CDCl}_{3}\right) \delta 3.50-3.48(\mathrm{~m}, 4 \mathrm{H}), 3.42-$ $3.40(\mathrm{~m}, 4 \mathrm{H}), 1.60-1.56(\mathrm{~m}, 12 \mathrm{H}) .{ }^{13} \mathrm{C} \mathrm{NMR}\left(125 \mathrm{MHz}, \mathrm{CDCl}_{3}\right) \delta 150.1$, 46.2, 45.3, 25.9, 25.3, 24.1. IR (neat) $v_{\max } 2936,2862,1748,1684,1402$, $1201 \mathrm{~cm}^{-1}$. HRMS[EI+] calcd for $\mathrm{C}_{12} \mathrm{H}_{20} \mathrm{~N}_{2} \mathrm{O}_{3}[\mathrm{M}]^{+}$240.1474, found

\footnotetext{
${ }^{1}$ Wei, W. T.; Zhu, W. M.; Ying, W. W.; Wu, Y.; Huang, Y. L.; Liang, H. Metal-Free Synthesis of Isatin Oximes via Radical Coupling Reactions of Oxindoles with t-BuONO in Water. Org. Biomol. Chem, 2017, 15, 5254.

${ }^{2}$ Rad, M. N. S.; Khalafi-Nezhad, A.; Babamohammadi, S.; Behrouz, S. Microwave-Assisted Three-Component Synthesis of Some Novel 1-Alkyl-1 $H$-indole-2,3-dione 3-(O-Alkyloxime) Derivatives as Potential Chemotherapeutic Agents. Helv. Chim. Acta, 2010, 93, 2454.
} 


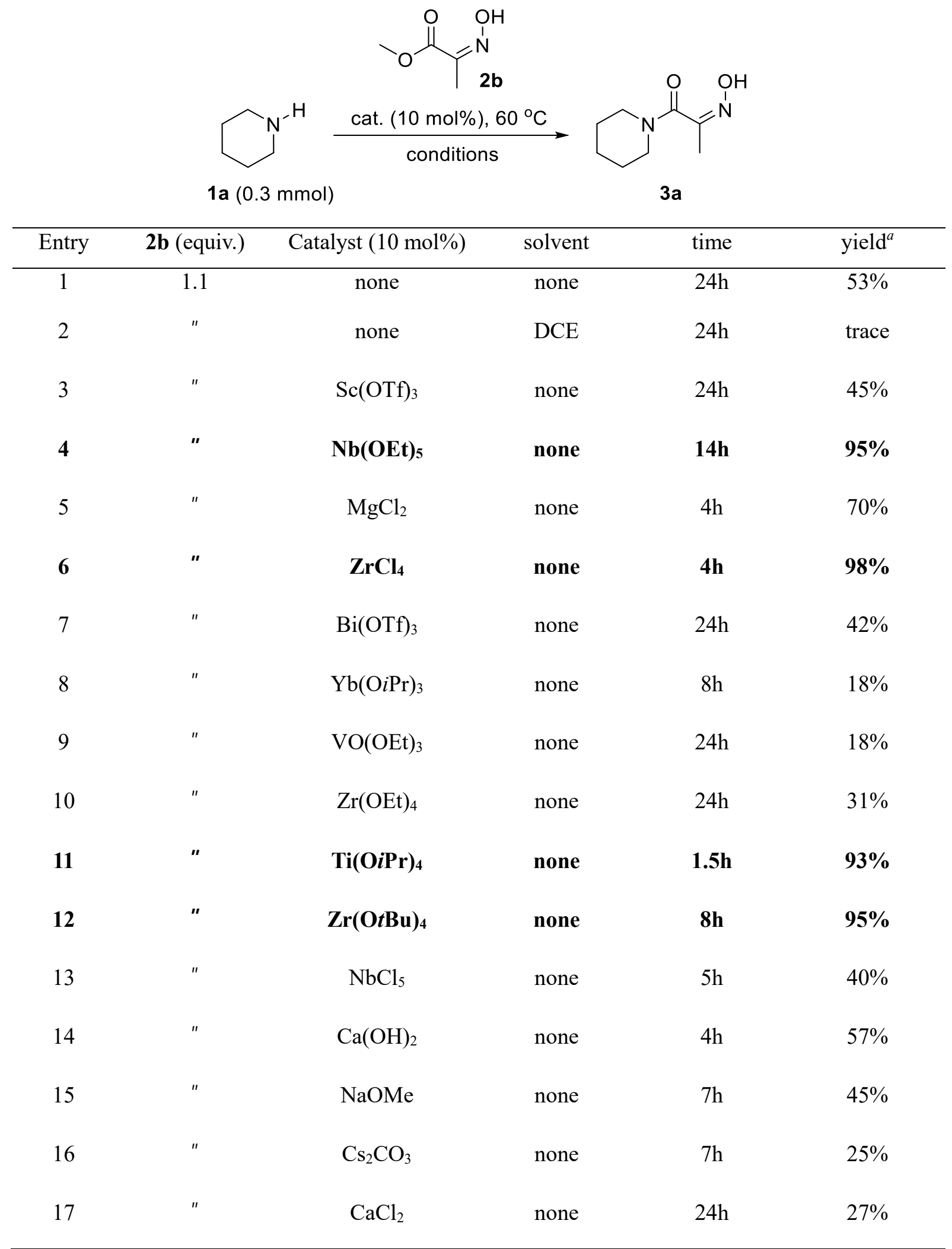

${ }^{a}$ NMR yield was determined with 1,3,5-trimethoxybenzene as the internal standard. 


\section{General Procedure for Lewis acid-assisted amidation}

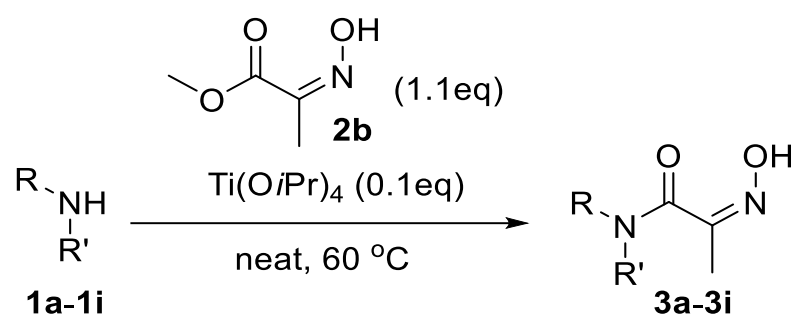

A mixture of amine $1(2.0 \mathrm{mmol})$, methyl pyruvate oxime $\mathbf{2 b}(258 \mathrm{mg}, 2.2 \mathrm{mmol})$, and titanium isopropoxide $(58 \mu \mathrm{L}, 0.2 \mathrm{mmol})$ in $5 \mathrm{ml}$ vial was heated at $60{ }^{\circ} \mathrm{C}$ in a pie-block for 2 hours. Then, the reaction mixture was cooled to room temperature. The crude product was recrystallized with ethyl acetate and hexane to afford $\mathbf{3 a - 3 i}$.

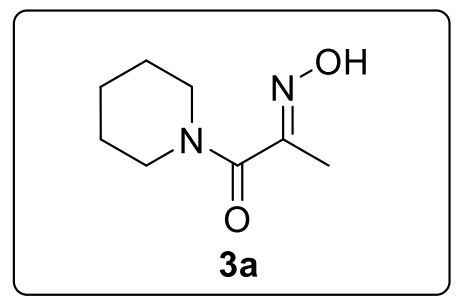

170.1064 .

2-(hydroxyimino)-1-(piperidin-1-yl)propan-1-one (3a); piperidine (170 mg, $2.0 \mathrm{mmol}$ ) was used to provide 3a (305 mg, 89\%); white solid; $\mathrm{R}_{f}=0.4(\mathrm{Hex}: \mathrm{EtOAc}=1: 2) ;{ }^{1} \mathrm{H}$ NMR $\left(300 \mathrm{MHz}, \mathrm{CDCl}_{3}\right) \delta 7.85(\mathrm{br} \mathrm{s}$, $1 \mathrm{H}), 3.59$ (t, $J=5.7 \mathrm{~Hz}, 2 \mathrm{H}), 3.47$ (t, $J=5.5 \mathrm{~Hz}, 2 \mathrm{H}), 2.07$ (br s, $3 \mathrm{H})$, 1.69-1.56 (m, 6H). ${ }^{13} \mathrm{C}$ NMR (100 MHz, $\left.\mathrm{CDCl}_{3}\right) \delta 165.3,152.2,48.0$, 43.1, 26.5, 25.4, 24.5, 12.5. IR (neat) $v_{\max } 3217,2943,1739,1588,1442$, $1365 \mathrm{~cm}^{-1}$. HRMS[EI+] calcd for $\mathrm{C}_{8} \mathrm{H}_{14} \mathrm{~N}_{2} \mathrm{O}_{2}[\mathrm{M}]^{+} 170.1055$, found

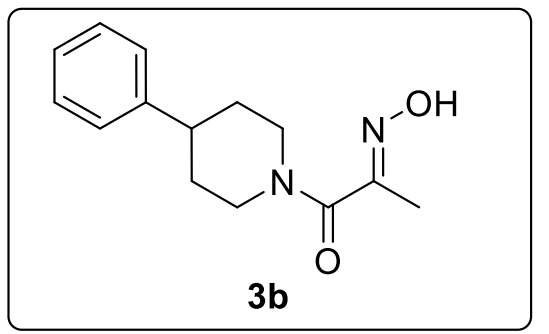

2-(hydroxyimino)-1-(4-phenylpiperidin-1-yl)propan-1-one (3b); 4-phenylpiperidine (322 $\mathrm{mg}, 2.0 \mathrm{mmol}$ ) was used to provide 3b (327 mg, 79\%); white solid; $\mathrm{R}_{f}=0.5($ Hex:EtOAc $=1: 2) ;{ }^{1} \mathrm{H}$ NMR $\left(500 \mathrm{MHz}, \mathrm{CDCl}_{3}\right) \delta 7.62($ br s, $1 \mathrm{H}), 7.31(\mathrm{t}, J=7.6 \mathrm{~Hz}$, $2 \mathrm{H}), 7.24-7.19(\mathrm{~m}, 3 \mathrm{H}), 4.76(\mathrm{~m}, 1 \mathrm{H}), 4.16(\mathrm{br} \mathrm{s}, 1 \mathrm{H}), 3.13$ (br s, $1 \mathrm{H}), 2.81-2.74(\mathrm{~m}, 2 \mathrm{H}), 2.11(\mathrm{~s}, 3 \mathrm{H}), 1.92-1.90(\mathrm{~m}, 2 \mathrm{H}), 1.73-1.65$ $(\mathrm{m}, 2 \mathrm{H}) .{ }^{13} \mathrm{C} \mathrm{NMR}\left(100 \mathrm{MHz}, \mathrm{CDCl}_{3}\right) \delta 165.3,152.4,145.0$, 128.6, 126.8, 126.6, 47.7, 42.8, 42.7, 33.9, 32.8, 12.5. IR (neat) $v_{\max } 3168,3061,2856,1616,1493,1370 \mathrm{~cm}^{-1}$. HRMS[EI+] calcd for $\mathrm{C}_{14} \mathrm{H}_{18} \mathrm{~N}_{2} \mathrm{O}_{2}[\mathrm{M}]^{+} 246.1368$, found 246.1356 .

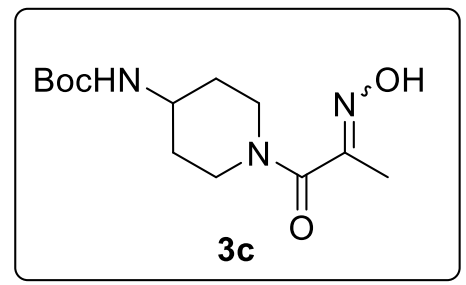

tert-butyl-(1-(2-(hydroxyimino)propanoyl)piperidin-4-yl)carbama te (3c); 4-(N-Boc-amino)piperidine (400 $\mathrm{mg}, 2.0 \mathrm{mmol})$ was used to provide 3c (462 mg, 94\%); white solid; $\mathrm{R}_{f}=0.4$ (Hex:EtOAc = 1:2); ${ }^{1} \mathrm{H}$ NMR $\left(400 \mathrm{MHz}, \mathrm{CDCl}_{3}\right.$, mixture of isomers, ratio 1:1) $\delta 10.66(\mathrm{br}$ $\mathrm{s}, 0.5 \mathrm{H}), 9.26(\mathrm{br} \mathrm{s}, 1 \mathrm{H}), 6.12(\mathrm{br} \mathrm{s}, 0.5 \mathrm{H}), 4.82(\mathrm{~m}, 1 \mathrm{H}), 4.44(\mathrm{~m}, 1 \mathrm{H})$, $4.36(\mathrm{~m}, 0.5 \mathrm{H}), 3.98(\mathrm{~m}, 0.5 \mathrm{H}), 3.95(\mathrm{~m}, 1 \mathrm{H}), 3.70(\mathrm{~s}, 1 \mathrm{H}), 3.52(\mathrm{~m}$,

$0.5 \mathrm{H}), 3.09(\mathrm{t}, J=12.8 \mathrm{~Hz}, 1.5 \mathrm{H}), 2.83(\mathrm{t}, J=8.8 \mathrm{~Hz}, 1.5 \mathrm{H}), 2.04(\mathrm{~s}, 4.5 \mathrm{H}), 1.99-1.88(\mathrm{~m}, 3.5 \mathrm{H}), 1.32-$ $1.28(\mathrm{~m}, 4.5 \mathrm{H}) .{ }^{13} \mathrm{C} \mathrm{NMR}\left(100 \mathrm{MHz}, \mathrm{CDCl}_{3}\right) \delta 165.4(2 \mathrm{C}), 157.1,155.3,152.0,151.5,81.1,80.0,48.5$, 47.8, 45.9, 45.4, 41.1, 40.7, 33.9, 33.2, 31.9, 31.4, 28.4 (2C), 12.4 (2C). IR (neat) $v_{\max } 3342,3205,2956$, $1696,1595,1508 \mathrm{~cm}^{-1} \mathrm{HRMS}[\mathrm{FAB}+]$ calcd for $\mathrm{C}_{13} \mathrm{H}_{24} \mathrm{~N}_{3} \mathrm{O}_{4}[\mathrm{M}+\mathrm{H}]^{+} 286.1767$, found 286.1760 . 


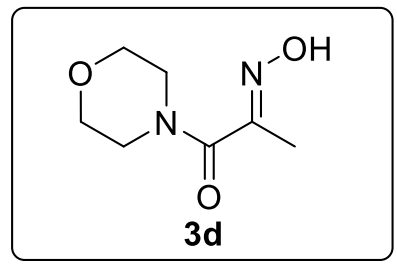

172.0838 .

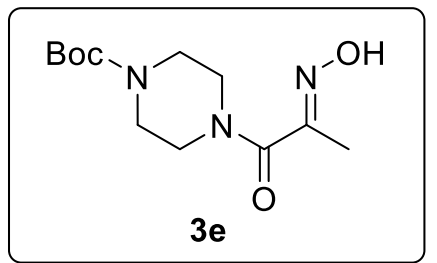

tert-butyl 4-(2-(hydroxyimino)propanoyl)piperazine-1-carboxylate (3e); 1-Boc-piperazine (372 $\mathrm{mg}, 2.0 \mathrm{mmol}$ ) was used to provide $\mathbf{3 e}$ (401 $\mathrm{mg}, 86 \%$ ); white solid; $\mathrm{R}_{f}=0.4$ (Hex:EtOAc $\left.=1: 2\right) ;{ }^{1} \mathrm{H} \mathrm{NMR}(400 \mathrm{MHz}$, $\left.\mathrm{CDCl}_{3}\right) \delta 9.12($ br s, $1 \mathrm{H}), 3.63-3.57(\mathrm{~m}, 4 \mathrm{H}), 3.46-3.42(\mathrm{~m}, 4 \mathrm{H}), 2.06(\mathrm{~s}$, $3 \mathrm{H}), 1.46(\mathrm{~s}, 9 \mathrm{H}) .{ }^{13} \mathrm{C} \mathrm{NMR}\left(100 \mathrm{MHz}, \mathrm{CDCl}_{3}\right) \delta 165.5,154.9,151.8$, $80.8,46.8,43.86,43.81,42.1,28.4,12.4$. IR (neat) $v_{\max } 3309,2863,1685$, $1608,1417,1165 \mathrm{~cm}^{-1}$. HRMS[EI+] calcd for $\mathrm{C}_{12} \mathrm{H}_{21} \mathrm{~N}_{3} \mathrm{O}_{4}[\mathrm{M}]^{+} 271.1532$, found 271.1531.

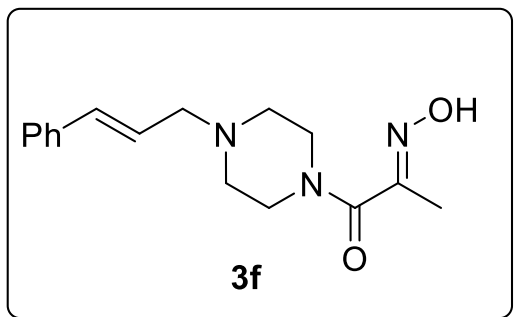

1-(4-cinnamylpiperazin-1-yl)-2-(hydroxyimino)propan-1-one (3f); $N$-cinnamylpiperazine (404 $\mathrm{mg}, 2.0 \mathrm{mmol}$ ) was used to provide 3f (465 mg, 94\%); off-white solid; $\mathrm{R}_{f}=0.4(10 \% \mathrm{MeOH}$ in DCM); ${ }^{1} \mathrm{H}$ NMR $\left(400 \mathrm{MHz}, \mathrm{CDCl}_{3}\right) \delta 10.43$ (br s, $\left.1 \mathrm{H}\right), 7.36-7.20$ $(\mathrm{m}, 5 \mathrm{H}), 6.49(\mathrm{~d}, J=15.8 \mathrm{~Hz}, 1 \mathrm{H}), 6.21(\mathrm{dt}, J=15.8,6.8 \mathrm{~Hz}, 1 \mathrm{H})$, $3.73(\mathrm{t}, J=5.1 \mathrm{~Hz}, 2 \mathrm{H}), 3.65(\mathrm{t}, J=5.1 \mathrm{~Hz}, 2 \mathrm{H}), 3.15(\mathrm{~d}, J=8.1$ $\mathrm{Hz}, 2 \mathrm{H}), 2.53(\mathrm{t}, J=5.0 \mathrm{~Hz}, 2 \mathrm{H}), 2.45(\mathrm{t}, J=5.0 \mathrm{~Hz}, 2 \mathrm{H}), 2.06(\mathrm{~s}$, $3 \mathrm{H}) .{ }^{13} \mathrm{C} \mathrm{NMR}\left(100 \mathrm{MHz}, \mathrm{CDCl}_{3}\right) \delta 165.5,151.4,136.5,134.3$, $128.7,127.9,126.5,124.9,60.8,53.2,52.5,46.5,41.6,12.4$. IR (neat) $v_{\max } 3021,2940,2632,1740$, $1616,1465,1357 \mathrm{~cm}^{-1}$. HRMS[EI+] calcd for $\mathrm{C}_{16} \mathrm{H}_{21} \mathrm{~N}_{3} \mathrm{O}_{2}[\mathrm{M}]^{+}$287.1634, found 287.1635.

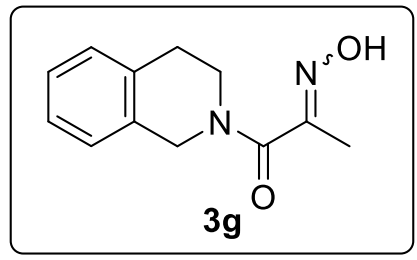

1-(3,4-dihydroisoquinolin-2(1H)-yl)-2-(hydroxyimino)propan-1-one (3g); 1,2,3,4-tetrahydroisoquinoline $(266 \mathrm{mg}, 2.0 \mathrm{mmol})$ was used to provide 3g (297 mg, 83\%); off-white solid; $\mathrm{R}_{f}=0.4$ (Hex:EtOAc $\left.=1: 1\right)$; ${ }^{1} \mathrm{H}$ NMR $\left(400 \mathrm{MHz}\right.$, DMSO- $d_{6}$, mixture of isomers, ratio 1:1.5) $\delta 11.43$ (br s, 0.4H), 11.41 (br s, 0.6H), 7.21-7.12 (m, 4H), $4.70(\mathrm{~s}, 0.8 \mathrm{H}), 4.66(\mathrm{~s}$, $1.2 \mathrm{H}), 3.73(\mathrm{t}, J=5.8 \mathrm{~Hz}, 2 \mathrm{H}), 2.85-2.80(\mathrm{~m}, 2 \mathrm{H}), 1.94(\mathrm{~s}, 1.8 \mathrm{H}), 1.91(\mathrm{~s}$, $1.2 \mathrm{H}) .{ }^{13} \mathrm{C}$ NMR $\left(100 \mathrm{MHz}, \mathrm{DMSO}-d_{6}\right) \delta 165.4,165.1,150.6,150.5,134.4,134.2,133.3,132.8,128.66$, $128.62,126.59,126.56,126.4,126.2,126.18,126.13,48.0$ (2C), 44.0, 43.8, 29.0, 27.6, 12.1, 12.0. IR (neat) $v_{\max } 3215,1739,1641,1487,1444,1366 \mathrm{~cm}^{-1}$. HRMS[FAB+] calcd for $\mathrm{C}_{12} \mathrm{H}_{15} \mathrm{~N}_{2} \mathrm{O}_{2}[\mathrm{M}+\mathrm{H}]^{+}$ 219.1134, found 219.1129 .

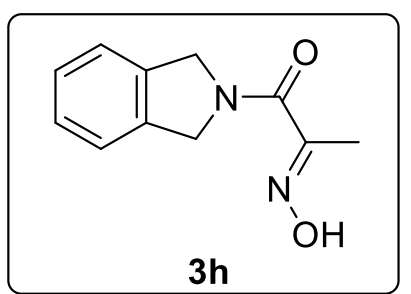

found 205.0993.

2-(hydroxyimino)-1-(isoindolin-2-yl)propan-1-one $\quad(3 \mathrm{~h}) ; \quad$ isoindoline (238 mg, $2.0 \mathrm{mmol}$ ) was used to provide $\mathbf{3 h}$ (346 mg, 85\%); brown solid; $\mathrm{R}_{f}=0.4(\mathrm{Hex}: \mathrm{EtOAc}=2: 1) ;{ }^{1} \mathrm{H} \mathrm{NMR}\left(400 \mathrm{MHz}, \mathrm{CDCl}_{3}\right) \delta 8.29(\mathrm{br} \mathrm{s}, 1 \mathrm{H})$, 7.31-7.25 (m, 3H), $7.19(\mathrm{~m}, 1 \mathrm{H}), 5.03(\mathrm{~s}, 2 \mathrm{H}), 4.90(\mathrm{~s}, 2 \mathrm{H}), 2.15(\mathrm{~s}, 3 \mathrm{H})$. ${ }^{13} \mathrm{C}$ NMR $\left(100 \mathrm{MHz}, \mathrm{CDCl}_{3}\right) \delta 164.4,153.7,137.0,135.5,127.7,127.6$, 122.9, 122.5, 54.8, 53.2, 11.5. IR (neat) $v_{\max } 3204,2863,1740,1641,1580$, 1443, $1352 \mathrm{~cm}^{-1}$. HRMS[FAB+] calcd for $\mathrm{C}_{11} \mathrm{H}_{13} \mathrm{~N}_{2} \mathrm{O}_{2}[\mathrm{M}]^{+}$205.0977, 


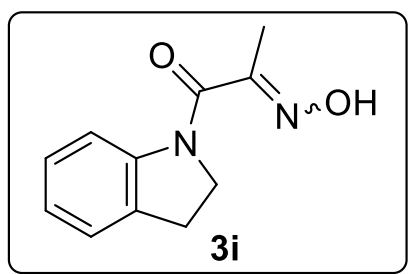

2-(hydroxyimino)-1-(indolin-1-yl)propan-1-one (3i); indoline $(238 \mathrm{mg}$, $2.0 \mathrm{mmol}$ ) was used to provide $3 \mathbf{i}(251 \mathrm{mg}, 76 \%)$; off-white solid; $\mathrm{R}_{f}=0.6$ (Hex:EtOAc $=2: 1) ;{ }^{1} \mathrm{H}$ NMR $\left(400 \mathrm{MHz}\right.$, acetone- $d_{6}$, mixture of isomers, ratio $1: 0.15$, minor isomer was not characterized) $\delta 10.76(\mathrm{br} \mathrm{s}, 1 \mathrm{H}), 8.14$ $(\mathrm{d}, J=7.64 \mathrm{~Hz}, 1 \mathrm{H}), 7.23-7.15(\mathrm{~m}, 2 \mathrm{H}), 7.02(\mathrm{t}, J=7.4 \mathrm{~Hz}, 1 \mathrm{H}), 4.24(\mathrm{t}, J$ $=8.4 \mathrm{~Hz}, 2 \mathrm{H}), 3.10(\mathrm{t}, J=8.4 \mathrm{~Hz}, 2 \mathrm{H}), 2.07(\mathrm{~s}, 3 \mathrm{H}) .{ }^{13} \mathrm{C} \mathrm{NMR}(100 \mathrm{MHz}$, acetone- $\left.d_{6}\right) \delta 164.8,153.2,143.9,133.5,127.7,125.5,124.7,118.0,52.3,28.7,11.4$. IR (neat) $v_{\max }$ $3299,1732,1621,1591,1482,1426 \mathrm{~cm}^{-1}$. HRMS[EI+] calcd for $\mathrm{C}_{11} \mathrm{H}_{12} \mathrm{~N}_{2} \mathrm{O}_{2}[\mathrm{M}]^{+}$204.0899, found 204.0892.

\section{Procedures for noncyclic secondary amides $(3 \mathbf{j}-3 \mathrm{l})$}<smiles>CN(C)C(=NO)N(C)C(=O)OCc1ccccc1</smiles>

(HATU, 1.2 eq)<smiles>On1nnc2ccccc21</smiles>

(HOBt, $1.2 \mathrm{eq})$

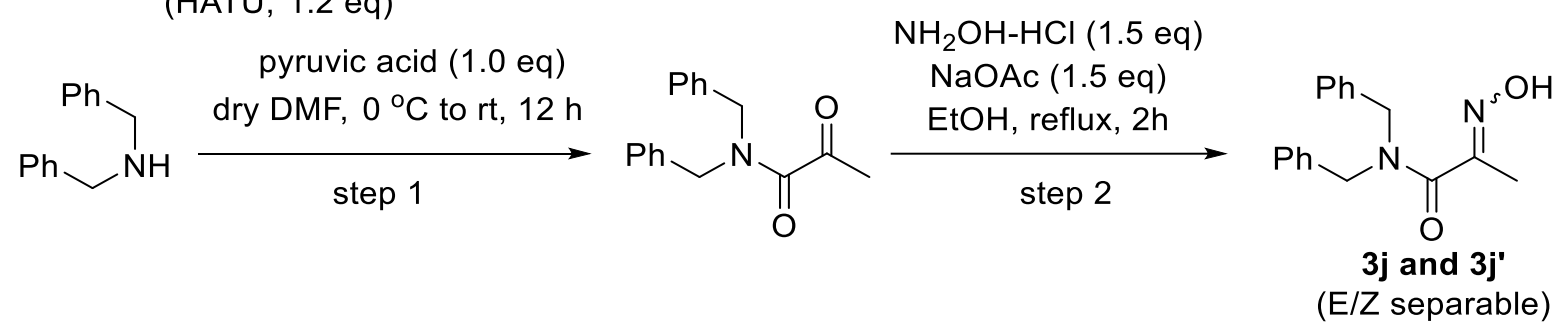

Step 1: To a stirred solution of pyruvic acid $(0.35 \mathrm{~mL}, 5.0 \mathrm{mmol})$ in dry DMF $(3.0 \mathrm{~mL})$ was added dibenzylamine $(0.95 \mathrm{~mL}, 5.0 \mathrm{mmol})$, a solution of 1-[Bis(dimethylamino)methylene]-1H-1,2,3triazolo[4,5-b]pyridinium 3-oxide hexafluorophosphate (HATU, $2.28 \mathrm{~g}, 6.0 \mathrm{mmol}$ ), and hydroxybenzotriazole (HOBt, $810 \mathrm{mg}, 6.0 \mathrm{mmol})$ in DMF $(6.0 \mathrm{~mL})$ under $0{ }^{\circ} \mathrm{C}$. The reaction mixture was then stirred for 12 hours, concentrated, diluted with EtOAc $(30 \mathrm{~mL})$, and washed with $1 \mathrm{~N} \mathrm{HCl}(6.0$ $\mathrm{mL})$ and brine $(6.0 \mathrm{~mL})$. The organic solution was dried over $\mathrm{MgSO}_{4}$, filtered, and concentrated. The residue was subjected to silica gel column chromatography (EtOAc:Hex $=1: 7$ to 1:5) to afford $N, N$ dibenzyl-2-oxopropanamide (414 mg, 31\%) as an oil; $\mathrm{R}_{f}=0.6$ (Hex:EtOAc $\left.=3: 1\right) ;{ }^{1} \mathrm{H}$ NMR $(400 \mathrm{MHz}$, $\left.\mathrm{CDCl}_{3}\right) \delta$ 7.38-7.31 (m, 6H), 7.26-7.19 (m, 4H), 4.55 (s, 2H), $4.40(\mathrm{~s}, 2 \mathrm{H}), 2.40$ (s, 3H). ${ }^{13} \mathrm{C} \mathrm{NMR}(100$ $\left.\mathrm{MHz}, \mathrm{CDCl}_{3}\right) \delta 198.6,167.5,135.8,135.6,129.0,128.9,128.5,128.2,127.9,127.8,50.0,47.2,27.8$. IR (neat) $v_{\max } 3063,3030,2926,1714,1641,1450,1355 \mathrm{~cm}^{-1}$. HRMS[EI+] calcd for $\mathrm{C}_{17} \mathrm{H}_{17} \mathrm{NO}_{2}[\mathrm{M}]^{+}$ 267.1259, found 267.1261.

Step 2: A mixture of the ketoamide obtained from step 1 (534 mg, $2.0 \mathrm{mmol}), \mathrm{NH}_{2} \mathrm{OH}-\mathrm{HCl}(206 \mathrm{mg}$, $3.0 \mathrm{mmol})$, and $\mathrm{NaOAc}(246 \mathrm{mg}, 3.0 \mathrm{mmol})$ in $\mathrm{EtOH}(6.0 \mathrm{~mL})$ was heated to reflux in heating mantle for 2 hours. Then, the reaction mixture was concentrated, diluted with EtOAc $(15 \mathrm{~mL})$, and washed with $\mathrm{H}_{2} \mathrm{O}(3 \mathrm{~mL})$. The organic solution was dried over $\mathrm{MgSO}_{4}$, filtered, and concentrated. The residue was subjected to silica gel column chromatography (EtOAc:Hex $=1: 7$ to $1: 4$ ) to afford $\mathbf{3 j}$ (344 $\mathrm{mg}$, $61 \%)$ and $\mathbf{3} \mathbf{j}^{\prime}$ (152 $\left.\mathrm{mg}, 27 \%\right)$.

(E)- $\mathbf{N}, \boldsymbol{N}$-dibenzyl-2-(hydroxyimino)propanamide (3j); yellow solid; $\mathrm{R}_{f}=0.5(\mathrm{Hex}: \operatorname{EtOAc}=1: 1) ;{ }^{1} \mathrm{H}$ NMR (400 MHz, $\left.\mathrm{CDCl}_{3}\right) \delta 8.51(\mathrm{br} \mathrm{s}, 1 \mathrm{H}), 7.39-7.26(\mathrm{~m}, 8 \mathrm{H}), 7.18(\mathrm{~d}, J=6.9 \mathrm{~Hz}, 2 \mathrm{H}), 4.64(\mathrm{~s}, 2 \mathrm{H})$, $4.36(\mathrm{~s}, 2 \mathrm{H}), 2.10(\mathrm{~s}, 3 \mathrm{H}) .{ }^{13} \mathrm{C} \mathrm{NMR}\left(100 \mathrm{MHz}, \mathrm{CDCl}_{3}\right) \delta 167.0,151.8,135.8,135.6,128.9,128.6$, 
128.2, 127.9, 127.7, 127.5, 50.2, 46.4, 17.4. IR (neat) $v_{\max } 3188,3061,2876,1740,1606,1429 \mathrm{~cm}^{-1}$. HRMS[EI+] calcd for $\mathrm{C}_{17} \mathrm{H}_{18} \mathrm{~N}_{2} \mathrm{O}_{2}[\mathrm{M}]^{+} 282.1368$, found 282.1342 .

(Z)-N,N-dibenzyl-2-(hydroxyimino)propanamide (3j'); white solid ; $\mathrm{R}_{f}=0.6(\mathrm{Hex}: \mathrm{EtOAc}=2: 1) ;{ }^{1} \mathrm{H}$ NMR $\left(400 \mathrm{MHz}, \mathrm{CDCl}_{3}\right) \delta 7.80(\mathrm{br} \mathrm{s}, 1 \mathrm{H}), 7.36-7.26(\mathrm{~m}, 6 \mathrm{H}), 7.23(\mathrm{~d}, J=1.8 \mathrm{~Hz}, 1 \mathrm{H}), 7.21(\mathrm{~d}, J=1.6$ $\mathrm{Hz}, 1 \mathrm{H}), 7.19(\mathrm{~d}, J=1.7 \mathrm{~Hz}, 1 \mathrm{H}), 7.17(\mathrm{~d}, J=2.5 \mathrm{~Hz}, 1 \mathrm{H}), 4.57(\mathrm{~s}, 2 \mathrm{H}), 4.53(\mathrm{~s}, 2 \mathrm{H}), 2.07(\mathrm{~s}, 3 \mathrm{H}) .{ }^{13} \mathrm{C}$ NMR $\left(100 \mathrm{MHz}, \mathrm{CDCl}_{3}\right) \delta 167.3,152.7,136.48,136.43,128.87,128.82,128.5$ (2C), 127.8, 127.7, 51.1, 47.5, 12.6. IR (neat) $v_{\max } 3331,3029,2970,1617,1359,1175 \mathrm{~cm}^{-1}$. HRMS[FAB+] calcd for $\mathrm{C}_{17} \mathrm{H}_{19} \mathrm{~N}_{2} \mathrm{O}_{2}[\mathrm{M}+\mathrm{H}]^{+}$283.1447, found 283.1429.

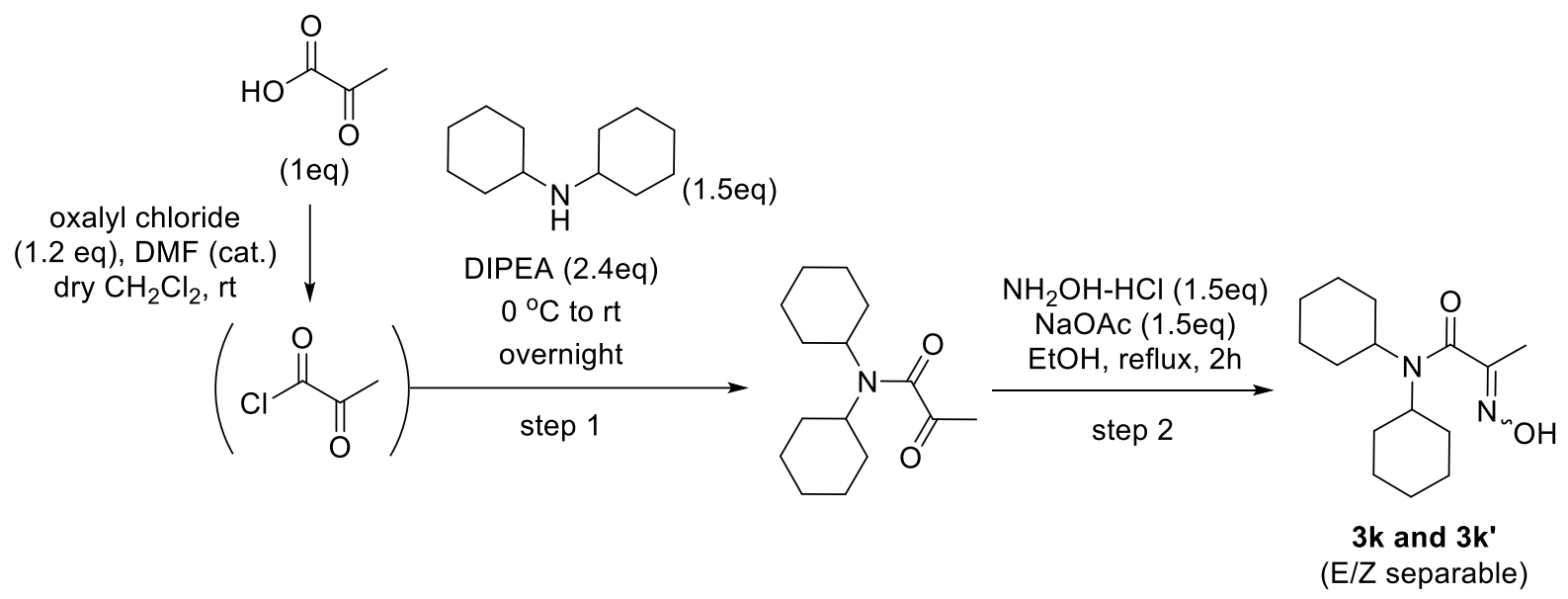

Step 1: To a stirred solution of pyruvic acid $(0.35 \mathrm{~mL}, 5.0 \mathrm{mmol})$ in dry $\mathrm{CH}_{2} \mathrm{Cl}_{2}(6.0 \mathrm{~mL})$ were added a catalytic amount of DMF (2 drops) and oxalyl chloride $(0.51 \mathrm{~mL}, 6.0 \mathrm{mmol})$. The reaction mixture was stirred at room temperature for 2 hours. Then, a solution of dicyclohexylamine $(1.48 \mathrm{~mL}, 7.5 \mathrm{mmol})$ and DIPEA $(2.0 \mathrm{~mL}, 12.0 \mathrm{mmol})$ in $\mathrm{CH}_{2} \mathrm{Cl}_{2}(1.0 \mathrm{~mL})$ was slowly added at $0{ }^{\circ} \mathrm{C}$. The reaction mixture was stirred at room temperature for $12 \mathrm{~h}$, diluted with water $(10 \mathrm{~mL})$, and extracted with $\mathrm{CH}_{2} \mathrm{Cl}_{2}(3 \mathrm{X}$ $10 \mathrm{~mL}$ ). The organic solution was dried over $\mathrm{MgSO}_{4}$, filtered, and concentrated. The residue was subjected to silica gel column chromatography (EtOAc:Hex $=1: 7$ to $1: 5)$ to afford N,N-dicyclohexyl2-oxopropanamide (239 mg, 19\%) as a white solid; $\mathrm{R}_{f}=0.5$ (Hex:EtOAc $\left.=10: 1\right) ;{ }^{1} \mathrm{H}$ NMR $(300 \mathrm{MHz}$, $\left.\mathrm{CDCl}_{3}\right) \delta 3.18(\mathrm{~m}, 1 \mathrm{H}), 3.05(\mathrm{~m}, 1 \mathrm{H}), 2.41-2.29(\mathrm{~m}, 4 \mathrm{H}), 1.82-1.73(\mathrm{~m}, 6 \mathrm{H}), 1.66-1.46(\mathrm{~m}, 6 \mathrm{H}), 1.43(\mathrm{~s}$, 1H), 1.30-1.15 (m, 5H), $1.08(\mathrm{~m}, 1 \mathrm{H}) .{ }^{13} \mathrm{C} \mathrm{NMR}\left(100 \mathrm{MHz}, \mathrm{CDCl}_{3}\right) \delta 198.9,167.8,58.6,55.7,31.3$, 29.6, 27.6, 26.4, 25.7, 25.2, 25.1. IR (neat) $v_{\max } 3007,2856,1740,1629,1444,1352,1177 \mathrm{~cm}^{-1}$. HRMS[EI+] calcd for $\mathrm{C}_{15} \mathrm{H}_{25} \mathrm{NO}_{2}[\mathrm{M}]^{+} 251.1885$, found 251.1897.

Step 2: A mixture of the ketoamide obtained from step 1 (503 mg, $2.0 \mathrm{mmol}), \mathrm{NH}_{2} \mathrm{OH}-\mathrm{HCl}(206 \mathrm{mg}$, $3.0 \mathrm{mmol})$, and $\mathrm{NaOAc}(246 \mathrm{mg}, 3.0 \mathrm{mmol})$ in $\mathrm{EtOH}(6.0 \mathrm{~mL})$ was heated to reflux in heating mantle for 2 hours. Then, the reaction mixture was concentrated, diluted with EtOAc $(15 \mathrm{~mL})$, and washed with $\mathrm{H}_{2} \mathrm{O}(3 \mathrm{~mL})$. The organic solution was dried over $\mathrm{MgSO}_{4}$, filtered, and concentrated. The residue was subjected to silica gel column chromatography (EtOAc:Hex $=1: 5$ to $1: 3)$ to afford $\mathbf{3 k}(287 \mathrm{mg}$, $54 \%$ ) and $\mathbf{3} \mathbf{k}^{\prime}$ (170 $\left.\mathrm{mg}, 32 \%\right)$.

(E)-N,N-dicyclohexyl-2-(hydroxyimino)propanamide (3k); white solid ; $\mathrm{R}_{f}=0.4(\operatorname{Hex}: \mathrm{EtOAc}=1: 1)$; ${ }^{1} \mathrm{H}$ NMR $\left(300 \mathrm{MHz}, \mathrm{CDCl}_{3}\right) \delta 7.80($ br s, $1 \mathrm{H}), 3.22(\mathrm{~m}, 1 \mathrm{H}), 2.96(\mathrm{~m}, 1 \mathrm{H}), 2.57-2.46(\mathrm{~m}, 2 \mathrm{H}), 1.98(\mathrm{~s}$, $3 \mathrm{H}), 1.81-1.77(\mathrm{~m}, 6 \mathrm{H}), 1.61-1.41(\mathrm{~m}, 6 \mathrm{H}), 1.33-1.04(\mathrm{~m}, 6 \mathrm{H}) .{ }^{13} \mathrm{C} \mathrm{NMR}\left(100 \mathrm{MHz}, \mathrm{CDCl}_{3}\right) \delta 165.4$, 153.0, 59.5, 55.8, 31.6, 29.7, 26.6 (2C), 25.8, 25.3, 17.5. IR (neat) $v_{\max } 3222,2928,1740,1602,1451$, $1374,1184 \mathrm{~cm}^{-1}$. HRMS[EI+] calcd for $\mathrm{C}_{15} \mathrm{H}_{26} \mathrm{~N}_{2} \mathrm{O}_{2}[\mathrm{M}]^{+}$266.1994, found 266.1996.

(Z)-N,N-dicyclohexyl-2-(hydroxyimino)propanamide (3k'); white solid; $\mathrm{R}_{f}=0.6$ (Hex:EtOAc $=2: 1$ ); ${ }^{1} \mathrm{H}$ NMR $\left(300 \mathrm{MHz}, \mathrm{CDCl}_{3}\right) \delta 8.40(\mathrm{br} \mathrm{s}, 1 \mathrm{H}), 3.48(\mathrm{~m}, 1 \mathrm{H}), 2.99(\mathrm{~m}, 1 \mathrm{H}), 2.44-2.41(\mathrm{~m}, 2 \mathrm{H}), 2.02(\mathrm{~s}$, 
$3 \mathrm{H}), 1.80-1.69(\mathrm{~m}, 6 \mathrm{H}), 1.59-1.37(\mathrm{~m}, 5 \mathrm{H}), 1.30-1.00(\mathrm{~m}, 7 \mathrm{H}) .{ }^{13} \mathrm{C}$ NMR $\left(100 \mathrm{MHz}, \mathrm{CDCl}_{3}\right) \delta 166.4$, 153.8, 59.4, 56.1, 31.3, 29.7, 26.6, 25.8, 25.3 (2C), 12.5. IR (neat) $v_{\max } 3235,2934,1740,1602,1449$, $1325,1199 \mathrm{~cm}^{-1}$. HRMS[EI+] calcd for $\mathrm{C}_{15} \mathrm{H}_{26} \mathrm{~N}_{2} \mathrm{O}_{2}[\mathrm{M}]^{+}$266.1994, found 266.1985.

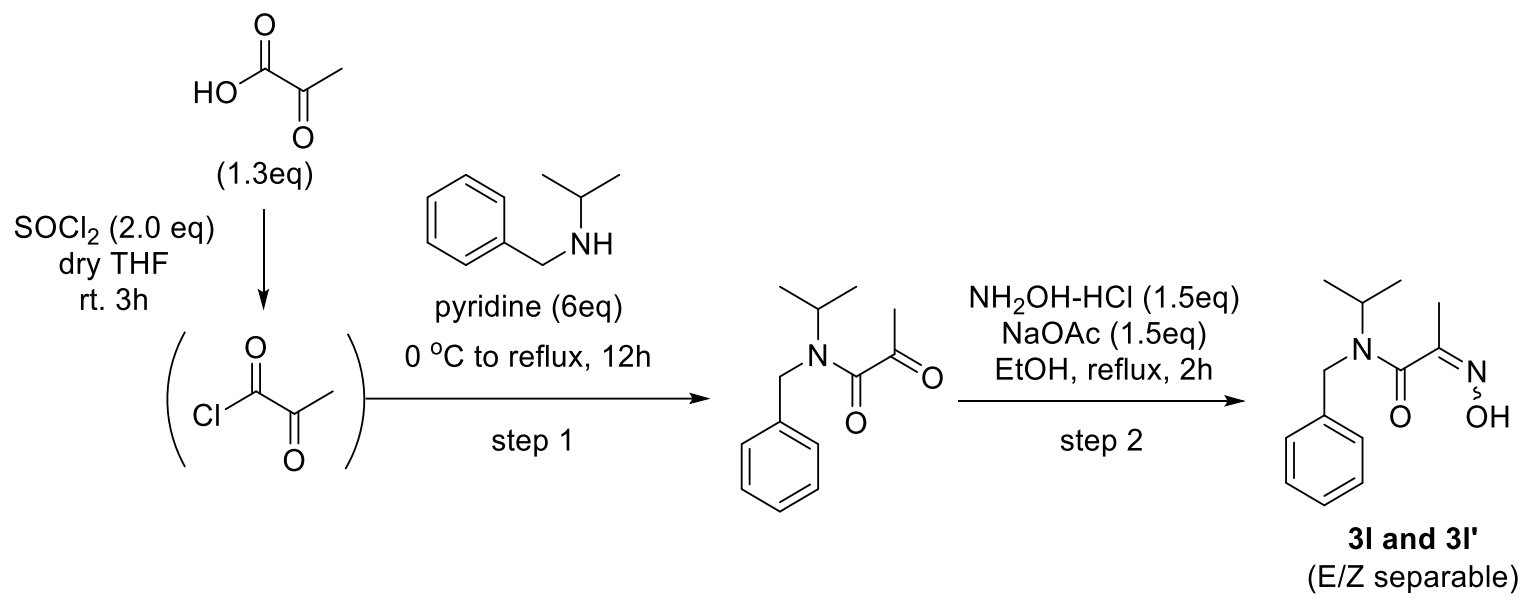

Step 1: To a stirred solution of pyruvic acid $(0.45 \mathrm{~mL}, 6.5 \mathrm{mmol})$ in dry THF $(15 \mathrm{~mL})$ was added thionyl chloride $(0.70 \mathrm{~mL}, 10.0 \mathrm{mmol})$. After being stirred for 3 hours at room temperature, a solution of $\mathrm{N}$ benzylpropan-2-amine $(0.82 \mathrm{~mL}, 5.0 \mathrm{mmol})$ and pyridine $(2.4 \mathrm{~mL}, 30 \mathrm{mmol})$ in THF $(2.0 \mathrm{~mL})$ was added to the reaction mixture at $0^{\circ} \mathrm{C}$, and the reaction mixture was heated to reflux for 12 hours. Then, it was quenched by adding $30 \mathrm{~mL}$ of $2 \mathrm{~N} \mathrm{HCl}$ solution and extracted with EtOAc $(30 \mathrm{~mL})$. The organic solution was dried over $\mathrm{MgSO}_{4}$, filtered, and concentrated. The residue was subjected to silica gel column chromatography (EtOAc:Hex $=1: 5$ to 1:3) to afford N-benzyl-N-isopropyl-2-oxopropanamide $(267 \mathrm{mg}, 23 \%)$ as brown oil; $\mathrm{R}_{f}=0.4(\mathrm{Hex}: \mathrm{EtOAc}=5: 1) ;{ }^{1} \mathrm{H} \mathrm{NMR}\left(400 \mathrm{MHz}, \mathrm{CDCl}_{3}\right.$, mixture of isomers, ratio 1:1) $\delta 7.34-7.20(\mathrm{~m}, 5 \mathrm{H}), 4.64(\mathrm{sept}, J=6.8 \mathrm{~Hz}, 0.5 \mathrm{H}), 4.56(\mathrm{~s}, 1 \mathrm{H}), 4.45(\mathrm{~s}, 1 \mathrm{H}), 3.99$ (sept, $J=6.6 \mathrm{~Hz}, 0.5 \mathrm{H}), 2.47(\mathrm{~s}, 1.5 \mathrm{H}), 2.01(\mathrm{~s}, 1.5 \mathrm{H}), 1.20(\mathrm{~d}, J=6.8 \mathrm{~Hz}, 3 \mathrm{H}), 1.15(\mathrm{~d}, J=6.6 \mathrm{~Hz}$, $3 \mathrm{H}) .{ }^{13} \mathrm{C} \mathrm{NMR}\left(100 \mathrm{MHz}, \mathrm{CDCl}_{3}\right) \delta 199.1,198.8,168.2,167.8,138.2,137.6,128.8,128.5,127.8,127.7$, 127.2, 127.1, 50.0, 46.7, 46.4, 43.4, 28.0, 26.8, 21.7, 20.1. IR (neat) $v_{\max } 2978,1713,1638,1450,1309$ $\mathrm{cm}^{-1}$. HRMS[EI+] calcd for $\mathrm{C}_{13} \mathrm{H}_{17} \mathrm{NO}_{2}[\mathrm{M}]^{+} 219.1259$, found 219.1250 .

Step 2: A mixture of the ketoamide obtained from step 1 (438 mg, $2.0 \mathrm{mmol}), \mathrm{NH}_{2} \mathrm{OH}-\mathrm{HCl}(206 \mathrm{mg}$, $3.0 \mathrm{mmol}$ ), and $\mathrm{NaOAc}(246 \mathrm{mg}, 3.0 \mathrm{mmol})$ in $\mathrm{EtOH}$ was heated to reflux in heating mantle for 2 hours. Then, the reaction mixture was concentrated, diluted with EtOAc $(15 \mathrm{~mL})$, and washed with $\mathrm{H}_{2} \mathrm{O}(3$ $\mathrm{mL}$ ). The organic solution was dried over $\mathrm{MgSO}_{4}$, filtered, and concentrated. The residue was subjected to silica gel column chromatography (EtOAc:Hex = 1:4 to 1:2) to afford $\mathbf{3 1}(241 \mathrm{mg}, 52 \%)$ and $\mathbf{3 \mathbf { I } ^ { \prime }}$ (148 $\mathrm{mg}, 32 \%)$.

(E)- $N$-benzyl-2-(hydroxyimino)- $N$-isopropylpropanamide (3I); white solid; $\mathrm{R}_{f}=0.5$ (Hex:EtOAc $=$ 2:1); ${ }^{1} \mathrm{H} \mathrm{NMR}\left(500 \mathrm{MHz}, \mathrm{CDCl}_{3}\right.$, mixture of isomers, ratio 1: 0.42) $\delta 9.21(\mathrm{br} \mathrm{s}, 1 \mathrm{H}), 7.33-7.18(\mathrm{~m}, 5 \mathrm{H})$, 4.64 (sept, $J=6.9 \mathrm{~Hz}, 0.3 \mathrm{H}), 4.61(\mathrm{~s}, 1.45 \mathrm{H}), 4.41(\mathrm{~s}, 0.55 \mathrm{H}), 3.94$ (sept, $J=6.6 \mathrm{~Hz}, 0.7 \mathrm{H}), 2.11$ (s, $2.2 \mathrm{H}), 1.71(\mathrm{~s}, 0.8 \mathrm{H}), 1.19(\mathrm{~d}, J=6.8 \mathrm{~Hz}, 1.8 \mathrm{H}), 1.11(\mathrm{~d}, J=6.6 \mathrm{~Hz}, 4.2 \mathrm{H}) .{ }^{13} \mathrm{C}$ NMR $\left(125 \mathrm{MHz}, \mathrm{CDCl}_{3}\right)$ $\delta 167.4,166.4,153.0,152.5,138.5,138.0,128.7,128.4,127.5,127.3,127.0,126.8,50.3,47.5,46.8$, 43.2, 21.9, 20.3, 17.6, 17.1. IR (neat) $v_{\max } 3241,3028,1607,1467,1420,1225 \mathrm{~cm}^{-1}$. HRMS[EI+] calcd for $\mathrm{C}_{13} \mathrm{H}_{18} \mathrm{~N}_{2} \mathrm{O}_{2}[\mathrm{M}]^{+}$234.1368, found 234.1375.

(Z)-N-benzyl-2-(hydroxyimino)-N-isopropylpropanamide (31'); yellow solid; $\mathrm{R}_{f}=0.7$ (Hex:EtOAc $=2: 1) ;{ }^{1} \mathrm{H}$ NMR $\left(500 \mathrm{MHz}, \mathrm{CDCl}_{3}\right.$, mixture of isomers, ratio 1: 0.8) $\delta 8.65(\mathrm{br} \mathrm{s}, 0.55 \mathrm{H}), 8.39$ (br s, $0.45 \mathrm{H}), 7.32-7.19(\mathrm{~m}, 5 \mathrm{H}), 4.69$ (sept, $J=6.9 \mathrm{~Hz}, 0.45 \mathrm{H}), 4.59$ (s, 1.1H), 4.55 (s, 0.9H), 4.32 (sept, $J=$ $6.8 \mathrm{~Hz}, 0.55 \mathrm{H}), 2.14(\mathrm{~s}, 1.65 \mathrm{H}), 1.69(\mathrm{~s}, 1.35 \mathrm{H}), 1.20(\mathrm{~d}, J=6.7 \mathrm{~Hz}, 2.7 \mathrm{H}), 1.13(\mathrm{~d}, J=6.7 \mathrm{~Hz}, 3.3 \mathrm{H})$. ${ }^{13} \mathrm{C}$ NMR $\left(125 \mathrm{MHz}, \mathrm{CDCl}_{3}\right) \delta 167.3(2 \mathrm{C}), 153.5,153.1,138.7,138.5,128.6,128.5,127.48,127.40$, 
127.0, 126.9, 50.5, 48.1, 46.9, 43.7, 21.7, 20.3, 12.7, 12.0. IR (neat) $v_{\max } 3192,3071,1599,1492,1361$, $1132 \mathrm{~cm}^{-1}$. HRMS[EI+] calcd for $\mathrm{C}_{13} \mathrm{H}_{18} \mathrm{~N}_{2} \mathrm{O}_{2}[\mathrm{M}]^{+}$234.1368, found 234.1375.

\section{General procedure for preparation of $5 \mathrm{a}-5 \mathrm{c}$}

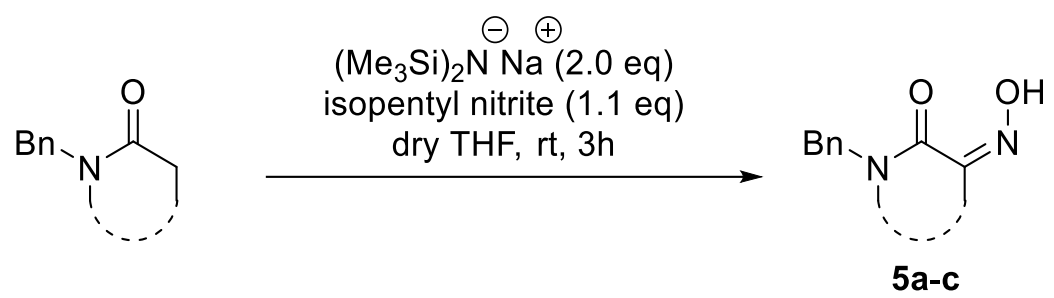

To a stirred solution of amide $(3.0 \mathrm{mmol})$ in dry THF $(15 \mathrm{~mL})$ was added sodium hexamethyldisilazide $(1.0 \mathrm{M}$ in THF, $6.0 \mathrm{~mL}, 6.0 \mathrm{mmol})$ at room temperature under Ar. After being stirred for $30 \mathrm{~min}$, isopentyl nitrite $(0.44 \mathrm{~mL}, 3.3 \mathrm{mmol})$ was added to the reaction mixture. After 3 hours, the reaction mixture was quenched with $1 \mathrm{~N} \mathrm{HCl}$ aq. sol'n $(15 \mathrm{~mL})$ and extracted with ethyl acetate $(3 \mathrm{X} 20 \mathrm{~mL})$. The organic solution was dried over $\mathrm{MgSO}_{4}$, filtered, and concentrated. The residue was subjected to silica gel column chromatography $(10 \% \mathrm{MeOH}$ in DCM) to afford 5a-c.

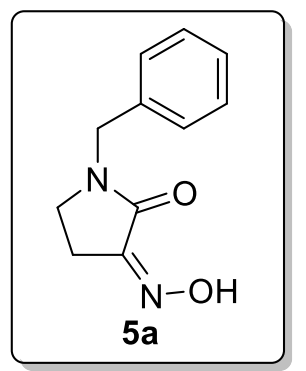

benzyl-3-(hydroxyimino)pyrrolidin-2-one (5a); 1-benzylpyrrolidin-2-one (525 $\mathrm{mg}, 3.0 \mathrm{mmol})$ was used to provide $\mathbf{5 a}(151 \mathrm{mg}, 25 \%)$ as brown solid; $\mathrm{R}_{f}=0.5$ $\left(10 \% \mathrm{MeOH}\right.$ in DCM); ${ }^{1} \mathrm{H}$ NMR $\left(500 \mathrm{MHz}\right.$, methanol- $\left.d_{4}\right) \delta 7.37-7.33(\mathrm{~m}, 2 \mathrm{H})$, 7.31-7.28 (m, 3H), $4.60(\mathrm{~s}, 2 \mathrm{H}), 3.41(\mathrm{t}, J=6.3 \mathrm{~Hz}, 2 \mathrm{H}), 2.81(\mathrm{t}, J=6.5 \mathrm{~Hz}, 2 \mathrm{H})$. ${ }^{13} \mathrm{C}$ NMR (100 MHz, methanol- $\left.d_{4}\right) \delta 166.0,152.9,137.0,129.9,129.2,128.9,48.1$, 44.0, 21.6. IR (neat) $v_{\max } 3064,2859,1678,1641,1452,1309 \mathrm{~cm}^{-1}$. HRMS[EI+] calcd for $\mathrm{C}_{11} \mathrm{H}_{12} \mathrm{~N}_{2} \mathrm{O}_{2}[\mathrm{M}]^{+}$204.0899, found 204.0884.

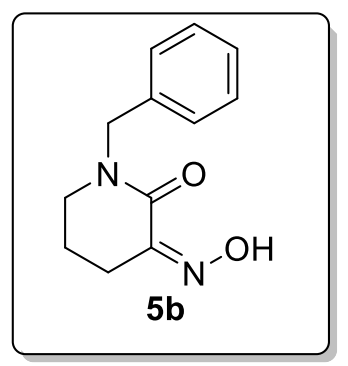

benzyl-3-(hydroxyimino)piperidin-2-one (5b); 1-benzylpiperidin-2-one (567 $\mathrm{mg}, 3.0 \mathrm{mmol})$ was used to provide $\mathbf{5 b}(98 \mathrm{mg}, 15 \%)$ as brown solid; $\mathrm{R}_{f}=0.5$ $\left(10 \% \mathrm{MeOH}\right.$ in DCM); ${ }^{1} \mathrm{H}$ NMR $\left(500 \mathrm{MHz}\right.$, methanol- $\left.d_{4}\right) \delta$ 7.35-7.26 (m, $\left.5 \mathrm{H}\right)$, 4.69 (s, 2H), 3.35 (t, $J=5.7 \mathrm{~Hz}, 2 \mathrm{H}$ ), 2.73 (t, $J=6.6 \mathrm{~Hz}, 2 \mathrm{H}$ ), 1.84 (quintet, $J$ $=6.4 \mathrm{~Hz}, 2 \mathrm{H}) .{ }^{13} \mathrm{C}$ NMR $\left(100 \mathrm{MHz}\right.$, methanol- $\left.d_{4}\right) \delta 163.2,150.7,138.0,129.7$, $129.0,128.6,51.6,47.7,24.0,22.0$. IR (neat) $v_{\max } 3133,2943,2345,1633,1575$, 1489, $1428 \mathrm{~cm}^{-1}$. HRMS[EI+] calcd for $\mathrm{C}_{12} \mathrm{H}_{14} \mathrm{~N}_{2} \mathrm{O}_{2}[\mathrm{M}]^{+} 218.1055$, found 218.1067 .

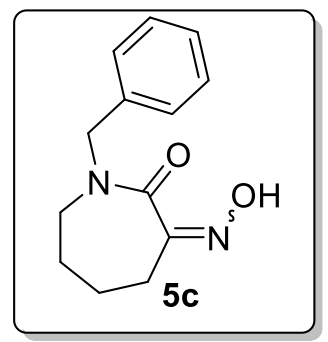

benzyl-3-(hydroxyimino)azepan-2-one (5c); 1-benzylazepan-2-one $(609 \mathrm{mg}$, $3.0 \mathrm{mmol})$ was a used to provide $\mathbf{5 c}(88 \mathrm{mg}, 13 \%)$ as brown solid; $\mathrm{R}_{f}=0.5(10 \%$ $\mathrm{MeOH}$ in DCM); ${ }^{1} \mathrm{H}$ NMR $\left(500 \mathrm{MHz}\right.$, methanol- $d_{4}$, mixture of isomers, ratio $1: 0.55) \delta 7.35-7.26(\mathrm{~m}, 5 \mathrm{H}), 4.64-4.63(\mathrm{~m}, 2 \mathrm{H}), 3.35(\mathrm{t}, J=5.8 \mathrm{~Hz}, 1.3 \mathrm{H}), 3.27$ (m, 0.7H), 2.55-2.53 (m, 0.7H), 2.39-2.36 (m, $1.3 \mathrm{H}), 1.77(\mathrm{~m}, J=6.1 \mathrm{~Hz}, 0.7 \mathrm{H})$, $1.67(\mathrm{~m}, J=6.3 \mathrm{~Hz}, 1.3 \mathrm{H}), 1.55-1.50(\mathrm{~m}, 2 \mathrm{H}) .{ }^{13} \mathrm{C}$ NMR $(100 \mathrm{MHz}$, methanol$\left.d_{4}\right) \delta 169.7,168.6,158.2,155.6,138.4,137.9,129.74,129.72,129.3,129.2$, $128.78,128.70,50.9,50.3,46.8(2 \mathrm{C}), 30.2,29.8,27.9,26.2,24.6,21.6$. IR (neat) $v_{\max } 3191,2868,1634,1598,1480,1444 \mathrm{~cm}^{-1}$. HRMS[EI+] calcd for $\mathrm{C}_{13} \mathrm{H}_{16} \mathrm{~N}_{2} \mathrm{O}_{2}[\mathrm{M}]^{+}$232.1212, found 232.1200 . 


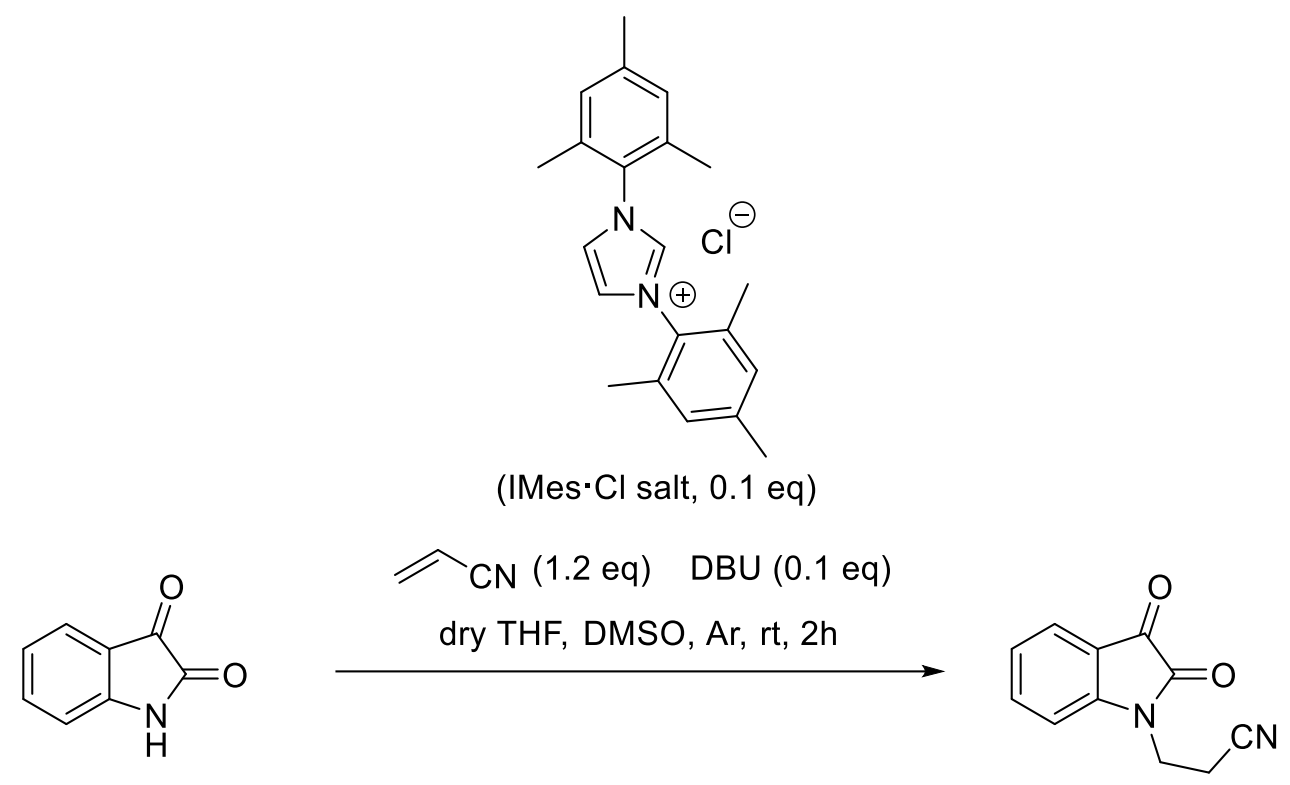

To a stirred solution of 1,3-bis(2,4,6-trimethylphenyl)imidazolinium chloride (102 $\mathrm{mg}, 0.3 \mathrm{mmol})$ and 1,8-diazabicyclo[5.4.0]undec-7-ene (DBU, $44 \mu \mathrm{L}, 0.3 \mathrm{mmol}$ ) in dry THF $(4 \mathrm{~mL})$ under Ar was added DMSO $(0.3 \mathrm{~mL})$ and acrylonitrile $(0.23 \mathrm{ml}, 3.6 \mathrm{mmol})$. Then, a solution of isatin $(441 \mathrm{mg}, 3.0 \mathrm{mmol})$ in dry THF $(4 \mathrm{~mL})$ was added dropwise at room temperature. After 2 hours, the reaction mixture was diluted with water $(5 \mathrm{~mL})$ and extracted with EtOAc $(3 \mathrm{X} 10 \mathrm{~mL})$. The organic solution was dried over $\mathrm{MgSO}_{4}$, filtered, and concentrated. The residue was subjected to silica gel column chromatography (EtOAc:Hex =1:7 to 1:2) to afford 3-(2,3-dioxoindolin-1-yl)propanenitrile (207 $\mathrm{mg}, 35 \%)$ as brown solid; $\mathrm{R}_{f}=0.4(\mathrm{Hex}: \mathrm{EtOAc}=1: 2) ;{ }^{1} \mathrm{H}$ NMR $\left(400 \mathrm{MHz}, \mathrm{CDCl}_{3}\right) \delta$ 7.67-7.63 (m, 2H), $7.18(\mathrm{t}, J=7.5$ $\mathrm{Hz}, 1 \mathrm{H}), 7.03(\mathrm{~d}, J=7.8 \mathrm{~Hz}, 1 \mathrm{H}), 4.05(\mathrm{t}, J=6.8 \mathrm{~Hz}, 2 \mathrm{H}), 2.82(\mathrm{t}, J=6.7 \mathrm{~Hz}, 2 \mathrm{H}) .{ }^{13} \mathrm{C}$ NMR $(100 \mathrm{MHz}$, $\left.\mathrm{CDCl}_{3}\right) \delta 182.2,158.3,149.7,138.8,126.1,124.6,117.8,117.0,110.0,36.4,16.7$. IR (neat) $v_{\max } 3452$, 3064, 2247, 2160, 1738, 1612, $1467 \mathrm{~cm}^{-1}$. HRMS[EI+] calcd for $\mathrm{C}_{11} \mathrm{H}_{8} \mathrm{~N}_{2} \mathrm{O}_{2}[\mathrm{M}]^{+}$200.0586, found 200.0584 .

\section{Conversion of ketoamides to 3-oximinoisatins 7a-71}

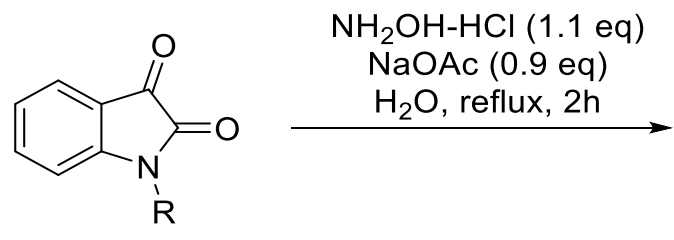<smiles>[R]N1C(=O)/C(=N/O)c2ccccc21</smiles>

A mixture of $N$-substituted isatin $(2.0 \mathrm{mmol})$ and hydroxylamine hydrochloride (152 $\mathrm{mg}, 2.2 \mathrm{mmol})$ in water $(6 \mathrm{~mL})$ was heated to reflux in heating mantle for $30 \mathrm{~min}$. Then, sodium acetate $(147 \mathrm{mg}, 1.8$ mmol) was added in and the solution was continued to reflux for another 2 hours. The mixture was cooled down to room temperature, diluted with water $(5 \mathrm{~mL})$, and extracted with EtOAc $(3 \mathrm{X} 10 \mathrm{~mL})$. The organic solution was dried over $\mathrm{MgSO}_{4}$, filtered, and concentrated. The residue was subjected to silica gel column chromatography to afford $\mathbf{7 a - 7 1}$. 

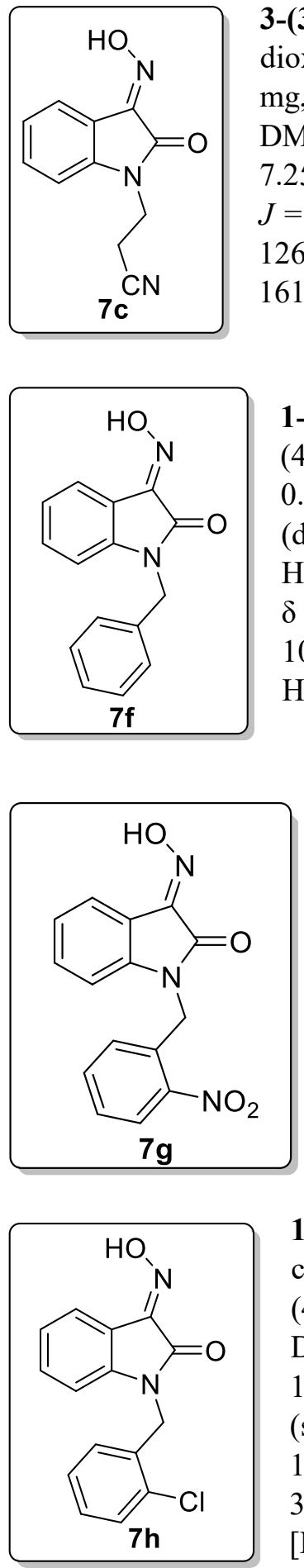

1-(2-chlorobenzyl)-3-(hydroxyimino)indolin-2-one

(7h);

$1-(2-$ chlorobenzyl)indoline-2,3-dione $(542 \mathrm{mg}, 2.0 \mathrm{mmol}$ ) was used to provide $7 \mathbf{h}$ $(439 \mathrm{mg}, 77 \%)$ as yellow soild; $\mathrm{R}_{f}=0.5(\mathrm{Hex}: \mathrm{EtOAc}=1: 1) ;{ }^{1} \mathrm{H}$ NMR $(400 \mathrm{MHz}$, DMSO- $\left.d_{6}\right) \delta 13.56($ br s, $1 \mathrm{H}), 8.04(\mathrm{dd}, J=7.5,1.2 \mathrm{~Hz}, 1 \mathrm{H}), 7.50(\mathrm{dd}, J=7.7$, $1.4 \mathrm{~Hz}, 1 \mathrm{H}), 7.35-7.26(\mathrm{~m}, 3 \mathrm{H}), 7.14-7.07(\mathrm{~m}, 2 \mathrm{H}), 6.86(\mathrm{~d}, J=7.8 \mathrm{~Hz}, 1 \mathrm{H}), 5.00$ $(\mathrm{s}, 2 \mathrm{H}) .{ }^{13} \mathrm{C}$ NMR $\left(100 \mathrm{MHz}, \mathrm{DMSO}-d_{6}\right) \delta 163.2,143.3,142.6,133.0,132.0$, $131.9,129.6,129.2,127.8,127.6,127.0,122.9,115.4,109.3,40.7$. IR (neat) $v_{\max }$ $3087,1730,1604,1463,1442,1342 \mathrm{~cm}^{-1}$. HRMS[EI+] calcd for $\mathrm{C}_{15} \mathrm{H}_{11} \mathrm{ClN}_{2} \mathrm{O}_{2}$ $[\mathrm{M}]^{+} 286.0509$, found 286.0511 .

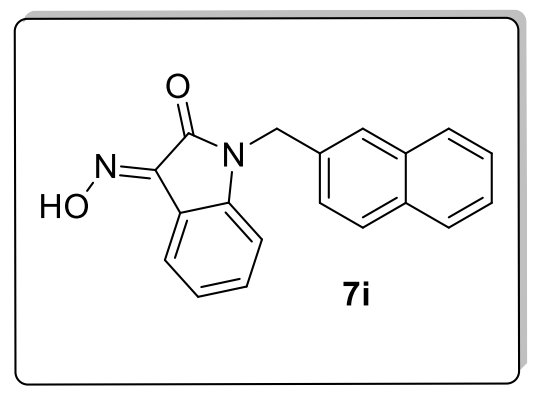

3-(hydroxyimino)-1-(naphthalen-2-ylmethyl)indolin-2-one (7i); 1-(naphthalen-1-ylmethyl)indoline-2,3-dione (574 mg, $2.0 \mathrm{mmol}$ ) was used to provide $7 \mathbf{i}(401 \mathrm{mg}, 67 \%)$ as yellow soild; $\mathrm{R}_{f}=0.4$ $($ Hex:EtOAc $=1: 1) ;{ }^{1} \mathrm{H}$ NMR $\left(400 \mathrm{MHz}\right.$, DMSO- $\left.d_{6}\right) \delta 13.57$ (br s, $1 \mathrm{H}), 8.23(\mathrm{~d}, J=8.4 \mathrm{~Hz}, 1 \mathrm{H}), 8.04(\mathrm{dd}, J=7.6,1.2 \mathrm{~Hz}, 1 \mathrm{H}), 7.97$ (dd, $J=7.8,1.6 \mathrm{~Hz}, 1 \mathrm{H}), 7.86(\mathrm{~d}, J=8.1 \mathrm{~Hz}, 1 \mathrm{H}), 7.64-7.55(\mathrm{~m}$, $2 \mathrm{H}), 7.42(\mathrm{t}, J=7.2 \mathrm{~Hz}, 1 \mathrm{H}), 7.32-7.27(\mathrm{~m}, 2 \mathrm{H}), 7.07(\mathrm{td}, J=7.6$, $0.9 \mathrm{~Hz}, 1 \mathrm{H}), 6.89(\mathrm{~d}, J=7.8 \mathrm{~Hz}, 1 \mathrm{H}), 5.43(\mathrm{~s}, 2 \mathrm{H}) .{ }^{13} \mathrm{C} \mathrm{NMR}(100$ MHz, DMSO- $\left.d_{6}\right) \delta 163.3,143.4,143.0,133.4,131.9,130.9,130.5$, 
128.7, 127.9, 126.9, 126.5, 126.0, 125.4, 124.0, 123.1, 122.8, 115.4, 109.8, 41.0. IR (neat) $v_{\max } 3043$, 2846, 1719, 1602, 1461, $1301 \mathrm{~cm}^{-1}$. HRMS[FAB+] calcd for $\mathrm{C}_{19} \mathrm{H}_{13} \mathrm{FN}_{2} \mathrm{O}[\mathrm{M}]^{+} 302.1055$, found 302.1062 .

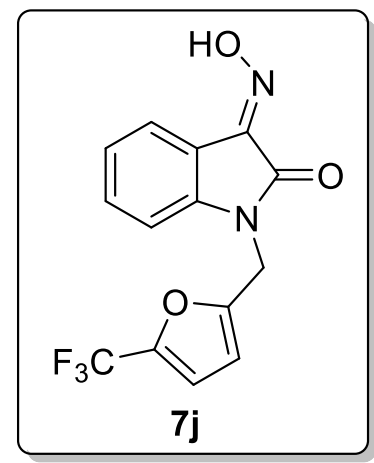

found 310.0579 .

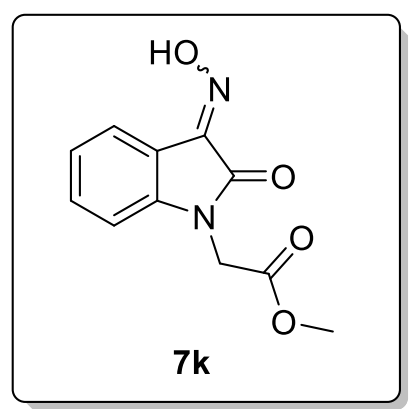

3-(hydroxyimino)-1-((5-(trifluoromethyl)furan-2-yl)methyl)indolin-2one (7j); 1-((5-(trifluoromethyl)furan-2-yl)methyl)indoline-2,3-dione (590 $\mathrm{mg}, 2.0 \mathrm{mmol}$ ) was used to provide $7 \mathbf{j}(364 \mathrm{mg}, 59 \%)$ as a yellow soild; $\mathrm{R}_{f}=$ $0.5(\mathrm{Hex}:$ EtOAc $=1: 1) ;{ }^{1} \mathrm{H}$ NMR $\left(400 \mathrm{MHz}, \mathrm{DMSO}-d_{6}\right) \delta 13.56($ br s, $1 \mathrm{H})$, $8.01(\mathrm{dd}, J=7.6,1.2 \mathrm{~Hz}, 1 \mathrm{H}), 7.42(\mathrm{td}, J=7.8,1.3 \mathrm{~Hz}, 1 \mathrm{H}), 7.18-7.14(\mathrm{~m}$, $2 \mathrm{H}), 7.10(\mathrm{td}, J=7.5,0.9 \mathrm{~Hz}, 1 \mathrm{H}), 6.70(\mathrm{~d}, J=3.4 \mathrm{~Hz}, 1 \mathrm{H}), 5.05(\mathrm{~s}, 2 \mathrm{H}) .{ }^{13} \mathrm{C}$ NMR $\left(100 \mathrm{MHz}, \mathrm{DMSO}-d_{6}\right) \delta 162.7,153.1\left(\mathrm{q}, J_{\mathrm{C}, \mathrm{F}}=1.5 \mathrm{~Hz}\right), 143.1,142.2$, $139.5\left(\mathrm{q}, J_{\mathrm{C}, \mathrm{F}}=41 \mathrm{~Hz}\right), 132.0,126.9,123.0,118.9\left(\mathrm{q}, J_{\mathrm{C}, \mathrm{F}}=264 \mathrm{~Hz}\right), 115.3$, $114.0\left(\mathrm{q}, J_{\mathrm{C}, \mathrm{F}}=2.8 \mathrm{~Hz}\right), 109.9,109.3,35.8$. IR (neat) $v_{\max } 3251,2361,1718$, $1614,1453,1318 \mathrm{~cm}^{-1}$. HRMS[EI+] calcd for $\mathrm{C}_{14} \mathrm{H}_{9} \mathrm{~F}_{3} \mathrm{~N}_{2} \mathrm{O}_{3}[\mathrm{M}]^{+} 310.0565$,

Methyl 2-(3-(hydroxyimino)-2-oxoindolin-1-yl)acetate (7k); methyl 2(2,3-dioxoindolin-1-yl)acetate ( $438 \mathrm{mg}, 2.0 \mathrm{mmol}$ ) was used to provide $7 \mathbf{k}$ $(313 \mathrm{mg}, 67 \%)$ as yellow soild; $\mathrm{R}_{f}=0.4$ (Hex:EtOAc $\left.=1: 1\right) ;{ }^{1} \mathrm{H}$ NMR $(400$ MHz, acetone- $d_{6}$, mixture of isomers, ratio 1:1) $\delta 12.49$ (br s, $\left.1 \mathrm{H}\right), 8.14(\mathrm{~d}$, $J=0.6 \mathrm{~Hz}, 0.5 \mathrm{H}), 8.12(\mathrm{~d}, J=0.6 \mathrm{~Hz}, 0.5 \mathrm{H}), 7.43(\mathrm{td}, J=7.8,1.3 \mathrm{~Hz}, 1 \mathrm{H})$, $7.13(\mathrm{td}, J=7.6,0.9 \mathrm{~Hz}, 1 \mathrm{H}), 7.04(\mathrm{t}, J=0.8 \mathrm{~Hz}, 0.5 \mathrm{H}), 7.02(\mathrm{t}, J=0.7 \mathrm{~Hz}$, $0.5 \mathrm{H}), 4.60(\mathrm{~s}, 2 \mathrm{H}), 3.73(\mathrm{~s}, 3 \mathrm{H}) .{ }^{13} \mathrm{C}$ NMR $\left(100 \mathrm{MHz}\right.$, acetone- $\left.d_{6}\right) \delta 169.2$, $168.9,164.1,164.0,144.9,144.8,144.4,144.2,132.99,132.98,128.4$, 128.3, 123.7, 123.6, $116.6(2 \mathrm{C}), 109.8(2 \mathrm{C}), 52.7$ (2C), 41.4, 41.3. IR (neat) $v_{\max } 3227,2418,1708,1605,1463,1345 \mathrm{~cm}^{-1}$. HRMS[EI+] calcd for $\mathrm{C}_{11} \mathrm{H}_{10} \mathrm{~N}_{2} \mathrm{O}_{4}[\mathrm{M}]^{+}$234.0641, found 234.0648 .

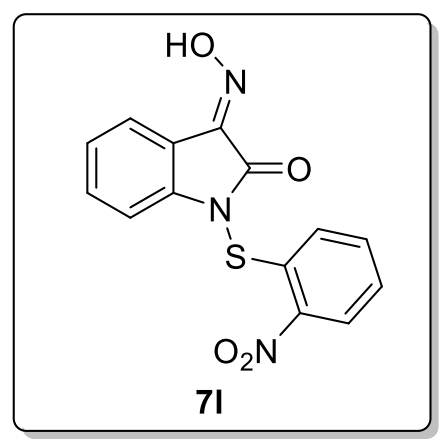

found 315.0313 . 3-(hydroxyimino)-1-((2-nitrophenyl)thio)indolin-2-one (71); 1-((2nitrophenyl)thio)indoline-2,3-dione $(600 \mathrm{mg}, 2.0 \mathrm{mmol})$ was used to provide 71 ( $318 \mathrm{mg}, 51 \%)$ as yellow soild; $\mathrm{R}_{f}=0.4(\mathrm{Hex}: \mathrm{EtOAc}=1: 1)$; ${ }^{1} \mathrm{H}$ NMR $\left(400 \mathrm{MHz}\right.$, DMSO- $\left.d_{6}\right) \delta 13.81(\mathrm{br} \mathrm{s}, 1 \mathrm{H}), 8.41$ (dd, $J=8.3,1.4$ $\mathrm{Hz}, 1 \mathrm{H}), 8.13(\mathrm{dd}, J=7.6,1.2 \mathrm{~Hz}, 1 \mathrm{H}), 7.71(\mathrm{td}, J=7.1,1.4 \mathrm{~Hz}, 1 \mathrm{H})$, $7.51(\mathrm{td}, J=7.1,1.2 \mathrm{~Hz}, 1 \mathrm{H}), 7.44(\mathrm{td}, J=7.9,1.3 \mathrm{~Hz}, 1 \mathrm{H}), 7.28-7.21$ $(\mathrm{m}, 2 \mathrm{H}), 7.16(\mathrm{~d}, J=7.8 \mathrm{~Hz}, 1 \mathrm{H}) .{ }^{13} \mathrm{C}$ NMR $\left(100 \mathrm{MHz}, \mathrm{DMSO}-d_{6}\right) \delta$ $164.9,142.87,142.80,142.6,137.9,135.8,132.4,127.1,126.9,126.3$, 124.3, 123.8, 117.3, 110.7. IR (neat) $v_{\max } 3100,2369,1736,1604,1511$, 1329, $1305 \mathrm{~cm}^{-1}$. HRMS[EI+] calcd for $\mathrm{C}_{14} \mathrm{H}_{9} \mathrm{~N}_{3} \mathrm{O}_{4} \mathrm{~S}[\mathrm{M}]^{+} 315.0314$, 


\section{General Procedure for preparation of carbamoyl fluorides}

To a stirred solution of the oxime $\mathbf{3}, \mathbf{5}$, or $7(0.3 \mathrm{mmol})$ in dry $\mathrm{CH}_{2} \mathrm{Cl}_{2}(\mathrm{DCM}, 1.0 \mathrm{~mL})$ was slowly added diethylaminosulfur trifluoride (DAST, $39 \mu \mathrm{L}, 0.3 \mathrm{mmol}$ ) at $0{ }^{\circ} \mathrm{C}$ under Ar. After $10 \mathrm{~min}$, the reaction mixture was diluted with DCM $(20 \mathrm{~mL})$ and washed with sat. $\mathrm{NaHCO}_{3}$ aq. sol'n $(5.0 \mathrm{~mL})$. The organic solution was dried over $\mathrm{MgSO}_{4}$, filtered, and concentrated. The crude mixture was subjected to silica gel column chromatography to afford the carbamoyl fluoride 4,6 , or 8 .

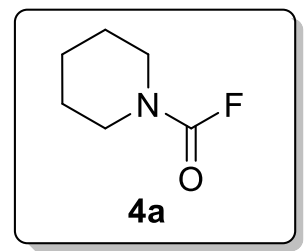

piperidine-1-carbonyl fluoride (4a); 3a (51 mg) was used to provide 4a (33 mg, $85 \%)$ as a colorless oil; $\mathrm{R}_{f}=0.4(\mathrm{Hex}: \mathrm{EtOAc}=7: 1) ;{ }^{1} \mathrm{H} \mathrm{NMR}\left(400 \mathrm{MHz}, \mathrm{CDCl}_{3}\right)$ $\delta 3.44-3.39(\mathrm{~m}, 4 \mathrm{H}), 1.64-1.57(\mathrm{~m}, 6 \mathrm{H}) .{ }^{19} \mathrm{~F} \mathrm{NMR}\left(376 \mathrm{MHz}, \mathrm{CDCl}_{3}\right) \delta-25.05(\mathrm{~s}$, $1 \mathrm{~F})$. The spectral data is in agreement with the known literature values. ${ }^{3}$

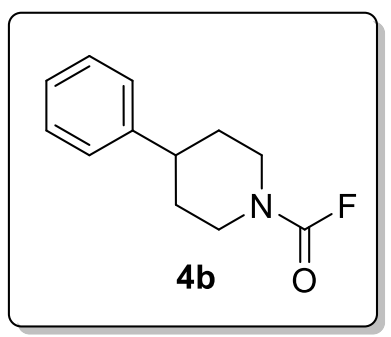

4-phenylpiperidine-1-carbonyl fluoride (4b); $3 \mathbf{b}(74 \mathrm{mg}$ ) was used to provide $4 \mathbf{b}(49 \mathrm{mg}, 79 \%)$ as white solid; $\mathrm{R}_{f}=0.4(\mathrm{Hex}: \mathrm{EtOAc}=5: 1) ;{ }^{1} \mathrm{H}$ NMR (300 MHz, $\left.\mathrm{CDCl}_{3}\right) \delta$ 7.35-7.30 (m, 2H), 7.26-7.18 (m, 3H), $4.21(\mathrm{~m}$, $1 \mathrm{H}), 4.09(\mathrm{~m}, 1 \mathrm{H}), 3.10-2.95(\mathrm{~m}, 2 \mathrm{H}), 2.71(\mathrm{tt}, J=12.0,3.6 \mathrm{~Hz}, 1 \mathrm{H}), 1.95-$ $1.88(\mathrm{~m}, 2 \mathrm{H}), 1.82-1.68(\mathrm{~m}, 2 \mathrm{H}) .{ }^{19} \mathrm{~F}$ NMR $\left(376 \mathrm{MHz}, \mathrm{CDCl}_{3}\right) \delta-24.58(\mathrm{~s}$, $1 \mathrm{~F})$. The spectral data is in agreement with the known literature values. ${ }^{4}$

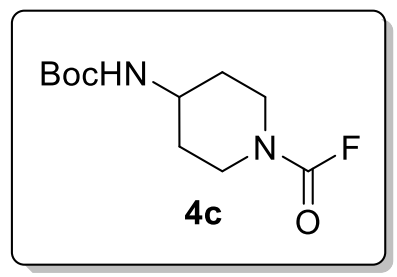

tert-butyl (1-(fluorocarbonyl)piperidin-4-yl)carbamate (4c); 3c (85 mg) was used to provide $4 \mathbf{c}(49 \mathrm{mg}, 67 \%)$ as white solid; $\mathrm{R}_{f}=0.4$ (Hex:EtOAc $=3: 1) ;{ }^{1} \mathrm{H} \mathrm{NMR}\left(400 \mathrm{MHz}, \mathrm{CDCl}_{3}\right) \delta 4.48(\mathrm{br} \mathrm{s}, 1 \mathrm{H}), 4.00(\mathrm{~d}, J=10.2,1 \mathrm{H})$, $3.89(\mathrm{~d}, J=10.7 \mathrm{~Hz}, 1 \mathrm{H}) 3.63(\mathrm{~s}, 1 \mathrm{H}), 3.08-2.98(\mathrm{~m}, 2 \mathrm{H}), 2.00(\mathrm{~m}, 2 \mathrm{H})$, 1.43-1.32 (m, 11H). ${ }^{19} \mathrm{~F}$ NMR $\left(376 \mathrm{MHz}, \mathrm{CDCl}_{3}\right) \delta-24.60(\mathrm{~s}, 1 \mathrm{~F})$. The spectral data is in agreement with the known literature values. ${ }^{3}$
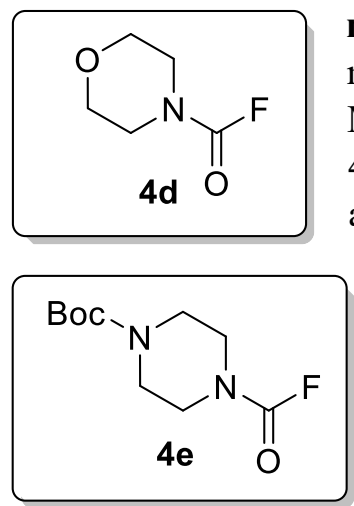

morpholine-4-carbonyl fluoride (4d); 3d (51 mg) was used to provide 4d (31 $\mathrm{mg}, 78 \%)$ as a white solid; yellow oil; $\mathrm{R}_{f}=0.3(\mathrm{Hex}:$ EtOAc $=3: 1) ;{ }^{1} \mathrm{H}$ NMR $(500$ $\left.\mathrm{MHz}, \mathrm{CDCl}_{3}\right) \delta 3.72(\mathrm{t}, J=4.6 \mathrm{~Hz}, 2 \mathrm{H}), 3.68(\mathrm{t}, J=4.8 \mathrm{~Hz}, 2 \mathrm{H}), 3.50-3.46(\mathrm{~m}$, $4 \mathrm{H}) .{ }^{19} \mathrm{~F}$ NMR $\left(376 \mathrm{MHz}, \mathrm{CDCl}_{3}\right) \delta-24.38(\mathrm{~s}, 1 \mathrm{~F})$. The spectral data is in agreement with the known literature values. ${ }^{5}$

tert-butyl 4-(fluorocarbonyl)piperazine-1-carboxylate (4e); run in $0.1 \mathrm{M}$ solution; 3e (81 mg) was used to provide $4 \mathbf{e}(41 \mathrm{mg}, 60 \%)$ as white solid; $\mathrm{R}_{f}$ $=0.4(\mathrm{Hex}: \mathrm{EtOAc}=5: 1) ;{ }^{1} \mathrm{H} \mathrm{NMR}\left(500 \mathrm{MHz}, \mathrm{CDCl}_{3}\right) \delta 3.47-3.44(\mathrm{~m}, 8 \mathrm{H})$, $1.46(\mathrm{~s}, 9 \mathrm{H}) .{ }^{19} \mathrm{~F}$ NMR $\left(376 \mathrm{MHz}, \mathrm{CDCl}_{3}\right) \delta-23.57(\mathrm{~s}, 1 \mathrm{~F})$. The spectral data is in agreement with the known literature values. ${ }^{3}$

\footnotetext{
3 Onida, K.; Tlili, A. Direct Synthesis of Carbamoyl Fluorides by $\mathrm{CO}_{2}$ Deoxyfluorination. Angew. Chem. Int. Ed. 2019, 58, 12545 .

4 Turksoy, A.; Scattolin, T.; Bouayad-Gevais, S.; Schoenebeck, F. Facile Access to AgOCF 3 and Its New Applications as a Reservoir for $\mathrm{OCF}_{2}$ for the Direct Synthesis of $N-\mathrm{CF}_{3}$, Aryl or Alkyl Carbamoyl Fluorides. Chem. Eur. J. 2020, 26, 2183
} 


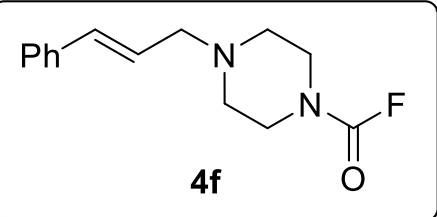

4-cinnamylpiperazine-1-carbonyl fluoride (4f); run in $0.1 \mathrm{M}$ solution; 3f (86 mg) was used to provide $4 \mathbf{f}(45 \mathrm{mg}, 61 \%)$ as white solid; $\mathrm{R}_{f}=0.5\left(3 \% \mathrm{MeOH}\right.$ in DCM); ${ }^{1} \mathrm{H}$ NMR $\left(300 \mathrm{MHz}, \mathrm{CDCl}_{3}\right) \delta$ 7.39-7.35 (m, 2H), 7.34-7.29 (m, 2H), 7.24 (m, 1H), $6.53(\mathrm{~d}, J=15.8$ $\mathrm{Hz}, 1 \mathrm{H}), 6.21(\mathrm{~m}, 1 \mathrm{H}), 3.52(\mathrm{dt}, J=8.9,5.0 \mathrm{~Hz}, 4 \mathrm{H}), 3.19(\mathrm{dd}, J=6.8$, $1.3 \mathrm{~Hz}, 2 \mathrm{H}), 2.56-2.49(\mathrm{~m}, 4 \mathrm{H}) .{ }^{19} \mathrm{~F}$ NMR $\left(376 \mathrm{MHz}, \mathrm{CDCl}_{3}\right) \delta-24.34(\mathrm{~s}, 1 \mathrm{~F})$. The spectral data is in agreement with the known literature values. ${ }^{3}$
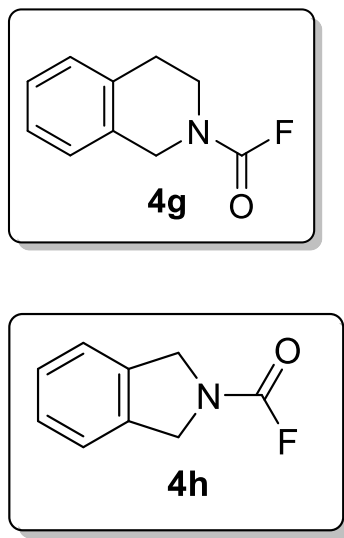

3,4-dihydroisoquinoline-2(1H)-carbonyl fluoride (4g); 3g (86 mg) was used to provide $4 \mathrm{~g}(38 \mathrm{mg}, 71 \%)$ as white solid; $\mathrm{R}_{f}=0.6(\mathrm{Hex}: \mathrm{EtOAc}=5: 1) ;{ }^{1} \mathrm{H}$ NMR $\left(400 \mathrm{MHz}, \mathrm{CDCl}_{3}\right.$, mixture of isomers, ratio 1:1) $\delta$ 7.26-7.09 $(\mathrm{m}, 4 \mathrm{H})$, $4.64(\mathrm{~s}, 1 \mathrm{H}), 4.63(\mathrm{~s}, 1 \mathrm{H}), 3.72(\mathrm{t}, J=5.9 \mathrm{~Hz}, 1 \mathrm{H}), 3.69(\mathrm{t}, J=4.6 \mathrm{~Hz}, 1 \mathrm{H}), 2.95-$ $2.90(\mathrm{~m}, 2 \mathrm{H}) .{ }^{19} \mathrm{~F}$ NMR $\left(376 \mathrm{MHz}, \mathrm{CDCl}_{3}\right) \delta-20.23$ (s, 0.5F), -23.19 (s, 0.5F). The spectral data is in agreement with the known literature values. ${ }^{3}$

isoindoline-2-carbonyl fluoride (4h); $3 \mathbf{h}(61 \mathrm{mg})$ was used to provide $4 \mathrm{~h}$ (33 $\mathrm{mg}, 68 \%)$ as yellow solid; $\mathrm{R}_{f}=0.5(\mathrm{Hex}:$ EtOAc $=10: 1) ;{ }^{1} \mathrm{H}$ NMR $(400 \mathrm{MHz}$, $\left.\mathrm{CDCl}_{3}\right) \delta$ 7.33-7.25 (m, 4H), $4.81(\mathrm{~s}, 2 \mathrm{H}), 4.79(\mathrm{~s}, 2 \mathrm{H}) .{ }^{13} \mathrm{C} \mathrm{NMR}(100 \mathrm{MHz}$ $\left.\mathrm{CDCl}_{3}\right) \delta 146.0\left(\mathrm{~d}, J_{\mathrm{C}, \mathrm{F}}=286 \mathrm{~Hz}\right), 135.44,135.40\left(\mathrm{~d}, J_{\mathrm{C}, \mathrm{F}}=1.4 \mathrm{~Hz}\right), 128.1(\mathrm{~d}$, $\left.J_{\mathrm{C}, \mathrm{F}}=4.2 \mathrm{~Hz}\right), 122.9\left(\mathrm{~d}, J_{\mathrm{C}, \mathrm{F}}=1.7 \mathrm{~Hz}\right), 122.8,52.8\left(\mathrm{~d}, J_{\mathrm{C}, \mathrm{F}}=47 \mathrm{~Hz}\right) .{ }^{19} \mathrm{~F}$ NMR $\left(376 \mathrm{MHz}, \mathrm{CDCl}_{3}\right) \delta-14.28$ (s, 1F). IR (neat) $v_{\max } 2941,2881,1783,1744,1464,1396 \mathrm{~cm}^{-1}$. HRMS[EI+] calcd for $\mathrm{C}_{9} \mathrm{H}_{8} \mathrm{FNO}[\mathrm{M}]^{+}$165.0590, found 165.0594.

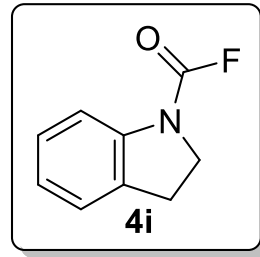

indoline-1-carbonyl fluoride (4i); $3 \mathbf{i}$ (61 mg) was used to provide $4 \mathbf{i}$ (19 $\mathrm{mg}, 38 \%$ ) as white solid; $\mathrm{R}_{f}=0.4(\mathrm{Hex}: \mathrm{EtOAc}=3: 1) ;{ }^{1} \mathrm{H} \mathrm{NMR}\left(500 \mathrm{MHz}, \mathrm{CDCl}_{3}\right.$, mixture of isomers, ratio 1: 0.4$) \delta 7.77(\mathrm{~d}, J=8.0 \mathrm{~Hz}, 0.7 \mathrm{H}), 7.32(\mathrm{dd}, J=8.3,4.5 \mathrm{~Hz}, 0.3 \mathrm{H})$, 7.27-7.20 (m, 2H), $7.07(\mathrm{~m}, 1 \mathrm{H}), 4.12-4.07(\mathrm{~m}, 2 \mathrm{H}), 3.22-3.18(\mathrm{~m}, 2 \mathrm{H}) .{ }^{19} \mathrm{~F}$ NMR $\left(376 \mathrm{MHz}, \mathrm{CDCl}_{3}\right) \delta-4.95(\mathrm{~s}, 0.7 \mathrm{~F}),-13.07(\mathrm{~s}, 0.3 \mathrm{~F})$. The spectral data is in agreement with the known literature values. ${ }^{5}$

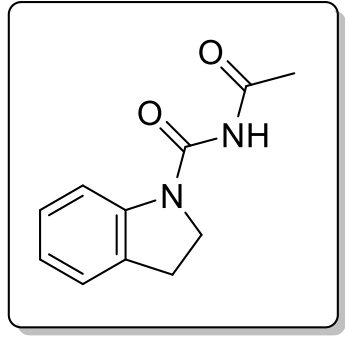

N-acetyl-2,3-dihydro-1H-indene-1-carboxamide; brown solid (16 mg, $26 \%) ; \mathrm{R}_{f}=0.4(\mathrm{Hex}: \mathrm{EtOAc}=1: 1) ;{ }^{1} \mathrm{H} \mathrm{NMR}\left(500 \mathrm{MHz}, \mathrm{CDCl}_{3}\right) \delta 8.26(\mathrm{~s}, 1 \mathrm{H})$, $7.89(\mathrm{~d}, J=7.8 \mathrm{~Hz}, 1 \mathrm{H}), 7.21-7.18(\mathrm{~m}, 2 \mathrm{H}), 7.01(\mathrm{t}, J=7.4 \mathrm{~Hz}, 1 \mathrm{H}), 4.07$ (t, $J$ $=8.4 \mathrm{~Hz}, 2 \mathrm{H}), 3.21(\mathrm{t}, J=8.5 \mathrm{~Hz}, 2 \mathrm{H}), 2.52(\mathrm{~s}, 3 \mathrm{H}) .{ }^{13} \mathrm{C} \mathrm{NMR}(100 \mathrm{MHz}$, $\left.\mathrm{CDCl}_{3}\right) \delta 173.8,150.1,142.2,131.3,127.8,125.0,123.7,115.9,47.9,27.9$, 24.8. IR (neat) $v_{\max } 3359,1698,1663,1479,1284,1019 \mathrm{~cm}^{-1}$. HRMS[EI+] calcd for $\mathrm{C}_{11} \mathrm{H}_{12} \mathrm{~N}_{2} \mathrm{O}_{2}[\mathrm{M}]^{+}$204.0899, found 204.0903.

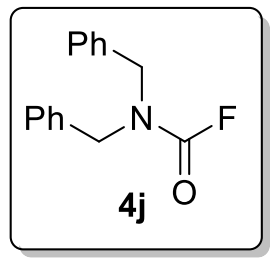

dibenzylcarbamic fluoride (4j); $\mathbf{3 j}$ (84 $\mathrm{mg}$ ) was used to provide $\mathbf{4 j}$ (68 $\mathrm{mg}, \mathbf{9 3} \%$ ) as yellow oil; $\mathrm{R}_{f}=0.7$ (Hex:EtOAc $\left.=7: 1\right) ;{ }^{1} \mathrm{H} \mathrm{NMR}\left(400 \mathrm{MHz}, \mathrm{CDCl}_{3}\right) \delta$ 7.40-7.32 $(\mathrm{m}, 6 \mathrm{H}), 7.29-7.25(\mathrm{~m}, 2 \mathrm{H}), 7.22-7.20(\mathrm{~m}, 2 \mathrm{H}), 4.42(\mathrm{~s}, 2 \mathrm{H}), 4.34(\mathrm{~s}, 2 \mathrm{H}) .{ }^{19} \mathrm{~F}$ NMR $\left(376 \mathrm{MHz}, \mathrm{CDCl}_{3}\right) \delta-22.60(\mathrm{~s}, 1 \mathrm{~F})$. The spectral data is in agreement with the known literature values. ${ }^{3}$

\footnotetext{
5 Song, H. X.; Han, Z. Z.; Zhang, C. P. Concise and Additive-Free Click Reactions between Amines and $\mathrm{CF}_{3} \mathrm{SO}_{3} \mathrm{CF}_{3}$. Chem. Eur. J. 2019, 25, 10907.
} 

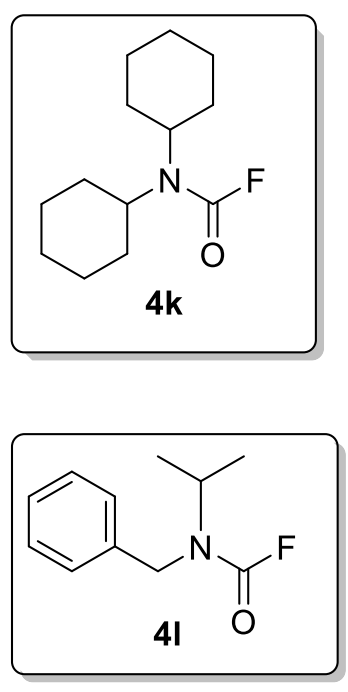

values. ${ }^{3}$

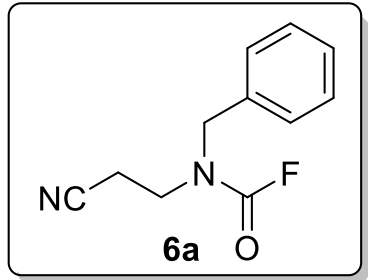

benzyl(2-cyanoethyl)carbamic fluoride (6a); 5a $(61 \mathrm{mg})$ was used to provide 6a (50 mg, 81\%) as yellow oil; $\mathrm{R}_{f}=0.4(\mathrm{Hex}:$ EtOAc $=1: 2) ;{ }^{1} \mathrm{H}$ NMR $\left(500 \mathrm{MHz}, \mathrm{CDCl}_{3}\right.$, mixture of isomers, ratio $\left.1: 0.66\right) \delta 7.41-7.30(\mathrm{~m}, 3.7 \mathrm{H})$, $7.25(\mathrm{~m}, 1.3 \mathrm{H}), 4.59(\mathrm{~s}, 0.8 \mathrm{H}), 4.57(\mathrm{~s}, 1.2 \mathrm{H}), 3.51(\mathrm{~m}, 1.2 \mathrm{H}), 3.47(\mathrm{~m}, 0.8 \mathrm{H})$, $2.62(\mathrm{t}, J=6.7 \mathrm{~Hz}, 1.2 \mathrm{H}), 2.50(\mathrm{t}, J=6.8 \mathrm{~Hz}, 0.8 \mathrm{H}) .{ }^{19} \mathrm{~F}$ NMR $(376 \mathrm{MHz}$, $\left.\mathrm{CDCl}_{3}\right) \delta-20.27(\mathrm{~s}, 0.4 \mathrm{~F}),-21.16(\mathrm{~s}, 0.6 \mathrm{~F})$. The spectral data is in agreement with the known literature values. ${ }^{6}$

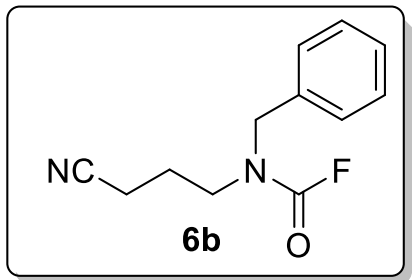

benzyl(3-cyanopropyl)carbamic fluoride (6b); $5 \mathbf{b}(65 \mathrm{mg})$ was used to provide $\mathbf{6 b}(60 \mathrm{mg}, 91 \%)$ as yellow oil; $\mathrm{R}_{f}=0.4(\mathrm{Hex}:$ EtOAc $=1: 2) ;{ }^{1} \mathrm{H}$ NMR (400 MHz, $\mathrm{CDCl}_{3}$, mixture of isomers, ratio 1:0.8) $\delta 7.40-7.29(\mathrm{~m}$, $4 \mathrm{H}), 7.25(\mathrm{~m}, 1 \mathrm{H}), 4.49(\mathrm{~s}, 0.9 \mathrm{H}), 4.45(\mathrm{~s}, 1.1 \mathrm{H}), 3.38(\mathrm{t}, J=7.1 \mathrm{~Hz}$, $1.1 \mathrm{H}), 3.31(\mathrm{t}, J=7.2 \mathrm{~Hz}, 0.9 \mathrm{H}), 2.32(\mathrm{~m}, 2 \mathrm{H}), 1.90(\mathrm{q}, J=7.2 \mathrm{~Hz}, 1.1 \mathrm{H})$, $1.84(\mathrm{q}, J=7.2 \mathrm{~Hz}, 0.9 \mathrm{H}) .{ }^{19} \mathrm{~F}$ NMR $\left(376 \mathrm{MHz}, \mathrm{CDCl}_{3}\right) \delta-21.048(\mathrm{~s}$, $0.55 \mathrm{~F}),-21.049(\mathrm{~s}, 0.45 \mathrm{~F}) .{ }^{13} \mathrm{C} \mathrm{NMR}\left(100 \mathrm{MHz}, \mathrm{CDCl}_{3}\right) \delta 147.9\left(\mathrm{~d}, J_{\mathrm{C}, \mathrm{F}}\right.$ $=286 \mathrm{~Hz}), 147.6\left(\mathrm{~d}, J_{\mathrm{C}, \mathrm{F}}=288 \mathrm{~Hz}\right), 135.4,135.3,129.2,129.1,128.58,128.55,128.2,127.6,118.7$, $118.4,52.3,51.8\left(\mathrm{~d}, J_{\mathrm{C}, \mathrm{F}}=3.4 \mathrm{~Hz}\right), 46.9,45.8\left(\mathrm{~d}, J_{\mathrm{C}, \mathrm{F}}=2.5 \mathrm{~Hz}\right), 24.2,23.2,14.7,14.6$. IR (neat) $v_{\max }$ 2946, 2247, 1786, 1422, 1219, $1057 \mathrm{~cm}^{-1} \mathrm{HRMS}[\mathrm{EI}+]$ calcd for $\mathrm{C}_{12} \mathrm{H}_{13} \mathrm{~N}_{2} \mathrm{O}[\mathrm{M}]^{+} 220.1012$, found 220.1019 .

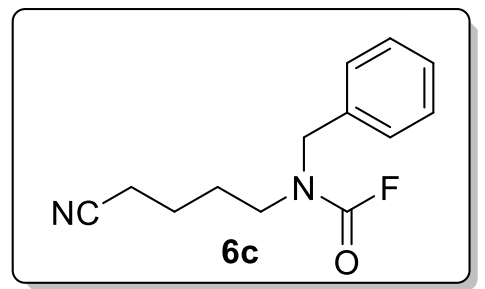

benzyl(4-cyanobutyl)carbamic fluoride (6c); 5 c (69 $\mathrm{mg}$ ) was used to provide $\mathbf{6 c}(59 \mathrm{mg}, 85 \%)$ as yellow oil; $\mathrm{R}_{f}=0.4(\mathrm{Hex}: \mathrm{EtOAc}=1: 3)$; ${ }^{1} \mathrm{H}$ NMR (400 MHz, $\mathrm{CDCl}_{3}$, mixture of isomers, ratio 1:0.9) $\delta$ 7.39$7.28(\mathrm{~m}, 3.8 \mathrm{H}), 7.24(\mathrm{~m}, 1.2 \mathrm{H}), 4.48(\mathrm{~s}, 0.9 \mathrm{H}), 4.43(\mathrm{~s}, 1.1 \mathrm{H}), 3.28(\mathrm{t}$, $J=6.8 \mathrm{~Hz}, 1.1 \mathrm{H}), 3.21(\mathrm{t}, J=6.7 \mathrm{~Hz}, 0.9 \mathrm{H}), 2.37-2.30(\mathrm{~m}, 2 \mathrm{H}), 1.74-$ $1.59(\mathrm{~m}, 4 \mathrm{H}) .{ }^{19} \mathrm{~F}$ NMR $\left(376 \mathrm{MHz}, \mathrm{CDCl}_{3}\right) \delta-21.50(\mathrm{~s}, 0.52 \mathrm{~F}),-21.99$ $(\mathrm{s}, 0.48 \mathrm{~F}) .{ }^{13} \mathrm{C}$ NMR $\left(100 \mathrm{MHz}, \mathrm{CDCl}_{3}\right) \delta 148.1\left(\mathrm{~d}, J_{\mathrm{C}, \mathrm{F}}=286 \mathrm{~Hz}\right)$, $147.7\left(\mathrm{~d}, J_{\mathrm{C}, \mathrm{F}}=287 \mathrm{~Hz}\right), 135.6,135.5,129.1,129.0,128.4,128.3,128.1,127.5,119.2,119.0,51.7$, $51.1\left(\mathrm{~d}, J_{\mathrm{C}, \mathrm{F}}=3.4 \mathrm{~Hz}\right), 46.8\left(\mathrm{~d}, J_{\mathrm{C}, \mathrm{F}}=1.5 \mathrm{~Hz}\right), 46.0\left(\mathrm{~d}, J_{\mathrm{C}, \mathrm{F}}=2.6 \mathrm{~Hz}\right), 27.2,26.1,22.5,22.4,16.9,16.8$. IR (neat) $v_{\max } 2945,2247,1785,1423,1220,1080 \mathrm{~cm}^{-1}$. HRMS[EI+] calcd for $\mathrm{C}_{13} \mathrm{H}_{15} \mathrm{FN}_{2} \mathrm{O}[\mathrm{M}]^{+}$

\footnotetext{
${ }^{6}$ Baars, H.; Engel, J.; Mertens, L.; Meister, D.; Bolm, C. The Reactivity of Difluorocarbene with Hydroxylamines: Synthesis of Carbamoyl Fluorides. Adv. Synth. Catal. 2016, 358, 2293.
} 
234.1168, found 234.1166.

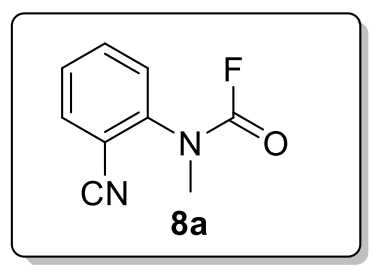

(2-cyanophenyl)(methyl)carbamic fluoride (8a); 7a (53 mg) was used to provide $8 \mathbf{a}(34 \mathrm{mg}, 64 \%)$ as yellow oil; $\mathrm{R}_{f}=0.5(\mathrm{Hex}: \mathrm{EtOAc}=1: 1) ;{ }^{1} \mathrm{H}$ NMR $\left(400 \mathrm{MHz}, \mathrm{CDCl}_{3}\right.$, mixture of isomers, ratio $\left.1: 2\right) \delta 7.74(\mathrm{dd}, J=7.8,1.5 \mathrm{~Hz}$, $1 \mathrm{H}), 7.69(\mathrm{~m}, 1 \mathrm{H}), 7.75-7.46(\mathrm{~m}, 1.3 \mathrm{H}), 7.40(\mathrm{~d}, J=8.0 \mathrm{~Hz}, 0.7 \mathrm{H}), 3.42(\mathrm{~d}, J$ $=1.0 \mathrm{~Hz}, 1 \mathrm{H}), 3.40(\mathrm{~d}, J=0.7 \mathrm{~Hz}, 2 \mathrm{H}) .{ }^{19} \mathrm{~F} \mathrm{NMR}\left(376 \mathrm{MHz}, \mathrm{CDCl}_{3}\right) \delta-13.30$ $(\mathrm{s}, 0.67 \mathrm{~F}),-17.84(\mathrm{~s}, 0.33 \mathrm{~F}) \cdot{ }^{13} \mathrm{C} \mathrm{NMR}\left(100 \mathrm{MHz}, \mathrm{CDCl}_{3}\right) \delta 146.4\left(\mathrm{~d}, J_{\mathrm{C}, \mathrm{F}}=\right.$ $291 \mathrm{~Hz}), 145.4\left(\mathrm{~d}, J_{\mathrm{C}, \mathrm{F}}=280 \mathrm{~Hz}\right), 144.0\left(\mathrm{~d}, J_{\mathrm{C}, \mathrm{F}}=6 \mathrm{~Hz}\right), 143.1\left(\mathrm{~d}, J_{\mathrm{C}, \mathrm{F}}=2 \mathrm{~Hz}\right), 134.39,134.37,134.0$, 133.9, 129.1, 128.9, 128.18, 128.14, 115.7, 115.5, 111.7, 111.6, 38.8 (d, $\left.J_{\mathrm{C}, \mathrm{F}}=2 \mathrm{~Hz}\right), 38.5$ (d, $J_{\mathrm{C}, \mathrm{F}}=3$ $\mathrm{Hz}$ ). IR (neat) $v_{\max } 2952,2232,1799,1598,1494,1372 \mathrm{~cm}^{-1}$. HRMS[EI+] calcd for $\mathrm{C}_{9} \mathrm{H}_{7} \mathrm{FN}_{2} \mathrm{O}[\mathrm{M}]^{+}$ 178.0542 , found 178.0550 .

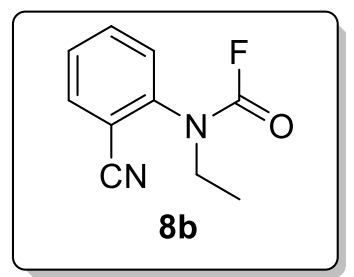

(2-cyanophenyl)(ethyl)carbamic fluoride (8b); $7 \mathbf{b}(57 \mathrm{mg})$ was used to provide $8 \mathbf{b}$ (42 mg, 73\%) as yellow oil; $\mathrm{R}_{f}=0.6(\mathrm{Hex}: \mathrm{EtOAc}=2: 1) ;{ }^{1} \mathrm{H} \mathrm{NMR}$ $\left(400 \mathrm{MHz}, \mathrm{CDCl}_{3}\right.$, mixture of isomers, ratio $\left.1: 2.3\right) \delta 7.76(\mathrm{dd}, J=7.6,1.6 \mathrm{~Hz}$, $1 \mathrm{H}), 7.68(\mathrm{~m}, 1 \mathrm{H}), 7.50(\mathrm{~m}, 1 \mathrm{H}), 7.42(\mathrm{~d}, J=8.0 \mathrm{~Hz}, 0.3 \mathrm{H}), 7.36(\mathrm{~d}, J=8 \mathrm{~Hz}$, $0.7 \mathrm{H}), 3.84-3.77(\mathrm{~m}, 2 \mathrm{H}), 1.28-1.22(\mathrm{~m}, 3 \mathrm{H}) .{ }^{19} \mathrm{~F} \mathrm{NMR}\left(376 \mathrm{MHz}, \mathrm{CDCl}_{3}\right) \delta$ $-11.63(\mathrm{~s}, 0.7 \mathrm{~F}),-19.86(\mathrm{~s}, 0.3 \mathrm{~F}) .{ }^{13} \mathrm{C} \mathrm{NMR}\left(100 \mathrm{MHz}, \mathrm{CDCl}_{3}\right) \delta 146.3\left(\mathrm{~d}, J_{\mathrm{C}, \mathrm{F}}\right.$ $=292 \mathrm{~Hz}), 145.0\left(\mathrm{~d}, J_{\mathrm{C}, \mathrm{F}}=287 \mathrm{~Hz}\right), 142.5\left(\mathrm{~d}, J_{\mathrm{C}, \mathrm{F}}=1.0 \mathrm{~Hz}\right), 141.5\left(\mathrm{~d}, J_{\mathrm{C}, \mathrm{F}}=1.8 \mathrm{~Hz}\right), 134.2,134.1,134.0$, $133.9,129.1(2 \mathrm{C}), 129.07,129.03,115.8,115.7,112.8(2 \mathrm{C}), 47.1\left(\mathrm{~d}, J_{\mathrm{C}, \mathrm{F}}=2.5 \mathrm{~Hz}\right), 47.0\left(\mathrm{~d}, J_{\mathrm{C}, \mathrm{F}}=2.6\right.$ $\mathrm{Hz}), 14.0,12.7$. IR (neat) $v_{\max } 2982,2232,1798,1598,1493,1454,1396 \mathrm{~cm}^{-1}$. HRMS[EI+] calcd for $\mathrm{C}_{10} \mathrm{H}_{9} \mathrm{FN}_{2} \mathrm{O}[\mathrm{M}]^{+}$192.0699, found 192.0697.

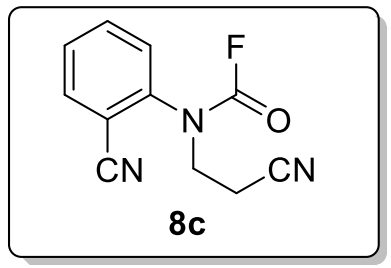

(2-cyanoethyl)(2-cyanophenyl)carbamic fluoride (8c); 7c (64 mg) was used to provide $8 \mathrm{c}(43 \mathrm{mg}, 66 \%)$ as brown solid; $\mathrm{R}_{f}=0.5(\mathrm{Hex}:$ EtOAc $=$ 2:1); ${ }^{1} \mathrm{H}$ NMR (400 MHz, $\mathrm{CDCl}_{3}$, mixture of isomers, ratio 1: 4) $\delta 7.80$ $7.73(\mathrm{~m}, 2 \mathrm{H}), 7.58-7.53(\mathrm{~m}, 2 \mathrm{H}), 4.06-4.03(\mathrm{~m}, 2 \mathrm{H}), 2.85(\mathrm{~s}, 1.6 \mathrm{H}), 2.75(\mathrm{t}$, $J=6.8 \mathrm{~Hz}, 0.4 \mathrm{H}) .{ }^{19} \mathrm{~F}$ NMR $\left(376 \mathrm{MHz}, \mathrm{CDCl}_{3}\right) \delta-11.02(\mathrm{~s}, 0.8 \mathrm{~F}),-17.65$ $(\mathrm{s}, 0.2 \mathrm{~F}) .{ }^{13} \mathrm{C}$ NMR $\left(100 \mathrm{MHz}, \mathrm{CDCl}_{3}\right) \delta 145.4\left(\mathrm{~d}, J_{\mathrm{C}, \mathrm{F}}=294 \mathrm{~Hz}\right), 145.0(\mathrm{~d}$, $\left.J_{\mathrm{C}, \mathrm{F}}=290 \mathrm{~Hz}\right), 141.6\left(\mathrm{~d}, J_{\mathrm{C}, \mathrm{F}}=1.7 \mathrm{~Hz}\right), 141.1\left(\mathrm{~d}, J_{\mathrm{C}, \mathrm{F}}=2.1 \mathrm{~Hz}\right), 134.76,134.71,134.2,134.0,129.99$, 129.96, 129.5, 129.3, 116.8, 116.3, 115.5, 115.4, 112.3, 112.2, $47.8\left(\mathrm{~d}, J_{\mathrm{C}, \mathrm{F}}=1.7 \mathrm{~Hz}\right), 47.2\left(\mathrm{~d}, J_{\mathrm{C}, \mathrm{F}}=1.4\right.$ $\mathrm{Hz}), 17.9,16.6$. IR (neat) $v_{\max } 2236,1791,1598,1488,1390,1268,1247 \mathrm{~cm}^{-1}$. HRMS[EI+] calcd for $\mathrm{C}_{11} \mathrm{H}_{8} \mathrm{FN}_{3} \mathrm{O}[\mathrm{M}]^{+}$217.0651, found 217.0654.

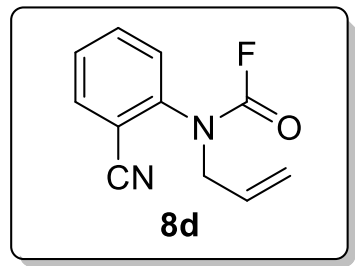

allyl(2-cyanophenyl)carbamic fluoride (8d); 7d $(61 \mathrm{mg})$ was used to provide 8d (53 mg, 87\%) as yellow oil; $\mathrm{R}_{f}=0.6(\mathrm{Hex}: \mathrm{EtOAc}=2: 1) ;{ }^{1} \mathrm{H} \mathrm{NMR}$ $\left(400 \mathrm{MHz}, \mathrm{CDCl}_{3}\right.$, mixture of isomers, ratio $\left.1: 2.3\right) \delta 7.73(\mathrm{dd}, J=7.8,1.7$ $\mathrm{Hz}, 1 \mathrm{H}), 7.67(\mathrm{~m}, 1 \mathrm{H}), 7.49(\mathrm{~m}, 1 \mathrm{H}), 7.42(\mathrm{~d}, J=8.0 \mathrm{~Hz}, 0.3 \mathrm{H}), 7.34(\mathrm{~d}, J=$ $8 \mathrm{~Hz}, 0.7 \mathrm{H}), 5.91(\mathrm{~m}, 1 \mathrm{H}), 5.26-5.15(\mathrm{~m}, 2 \mathrm{H}), 4.34-4.28(\mathrm{~m}, 2 \mathrm{H}) .{ }^{19} \mathrm{~F}$ NMR $\left(376 \mathrm{MHz}, \mathrm{CDCl}_{3}\right) \delta-12.05$ (s, 0.7F), $-18.63(\mathrm{~s}, 0.3 \mathrm{~F}) .{ }^{13} \mathrm{C} \mathrm{NMR}(100 \mathrm{MHz}$, $\left.\mathrm{CDCl}_{3}\right) \delta 146.1\left(\mathrm{~d}, J_{\mathrm{C}, \mathrm{F}}=293 \mathrm{~Hz}\right), 145.1\left(\mathrm{~d}, J_{\mathrm{C}, \mathrm{F}}=288 \mathrm{~Hz}\right), 142.5\left(\mathrm{~d}, J_{\mathrm{C}, \mathrm{F}}=1.4 \mathrm{~Hz}\right), 141.3\left(\mathrm{~d}, J_{\mathrm{C}, \mathrm{F}}=1.8\right.$ $\mathrm{Hz}), 134.1,134.0,133.9,133.8,131.1,130.5,129.2$ (2C), 129.1, 129.0, 120.9, 120.2, 115.8, 115.7, 112.7, 112.6, $54.5\left(\mathrm{~d}, J_{\mathrm{C}, \mathrm{F}}=2.1 \mathrm{~Hz}\right), 54.4\left(\mathrm{~d}, J_{\mathrm{C}, \mathrm{F}}=2.5 \mathrm{~Hz}\right)$. IR (neat) $v_{\max } 3085,2232,1798,1598,1493$,<smiles>Cc1ccccc1N(C(=O)F)c1ccccc1</smiles>

1390, $1265 \mathrm{~cm}^{-1}$. HRMS[EI+] calcd for $\mathrm{C}_{11} \mathrm{H}_{9} \mathrm{FN}_{2} \mathrm{O}[\mathrm{M}]^{+} 204.0699$, found 204.0699 .

(2-cyanophenyl)(phenyl)carbamic fluoride (8e); 7e (71 mg) was used to provide $8 \mathrm{e}(50 \mathrm{mg}, 70 \%)$ as brown solid; $\mathrm{R}_{f}=0.5(\mathrm{Hex}:$ EtOAc $=2: 1) ;{ }^{1} \mathrm{H}$ NMR $\left(400 \mathrm{MHz}, \mathrm{CDCl}_{3}\right.$, mixture of isomers, ratio 1: 1) $\delta 7.75(\mathrm{~d}, J=7.6 \mathrm{~Hz}, 1 \mathrm{H})$, $7.68(\mathrm{t}, J=7.7 \mathrm{~Hz}, 1 \mathrm{H}), 7.49(\mathrm{~d}, J=8.2 \mathrm{~Hz}, 2 \mathrm{H}), 7.43-7.32(\mathrm{~m}, 5 \mathrm{H}){ }^{19} \mathrm{~F}$ NMR $\left(376 \mathrm{MHz}, \mathrm{CDCl}_{3}\right) \delta-7.32(\mathrm{~s}, 0.5 \mathrm{~F}),-12.83(\mathrm{~s}, 0.5 \mathrm{~F}) .{ }^{13} \mathrm{C} \mathrm{NMR}(100 \mathrm{MHz}$, 
$\left.\mathrm{CDCl}_{3}\right) \delta 145.1\left(\mathrm{~d}, J_{\mathrm{C}, \mathrm{F}}=295 \mathrm{~Hz}\right), 144.2\left(\mathrm{~d}, J_{\mathrm{C}, \mathrm{F}}=290 \mathrm{~Hz}\right), 142.8\left(\mathrm{~d}, J_{\mathrm{C}, \mathrm{F}}=8.0 \mathrm{~Hz}\right), 142.5\left(\mathrm{~d}, J_{\mathrm{C}, \mathrm{F}}=80\right.$ $\mathrm{Hz}), 139.7\left(\mathrm{~d}, J_{\mathrm{C}, \mathrm{F}}=2.1 \mathrm{~Hz}\right), 139.4\left(\mathrm{~d}, J_{\mathrm{C}, \mathrm{F}}=51 \mathrm{~Hz}\right), 134.3(2 \mathrm{C}), 134.1(2 \mathrm{C}), 129.6,129.5,129.4,129.2$, $129.1,128.9,128.5,127.8,126.8,125.5,115.8,115.6,112.5,112.3$. IR (neat) $v_{\max } 2228,1792,1593$, $1488,1342,1200 \mathrm{~cm}^{-1}$. HRMS[EI+] calcd for $\mathrm{C}_{14} \mathrm{H}_{9} \mathrm{FN}_{2} \mathrm{O}[\mathrm{M}]^{+} 240.0699$, found 240.0700 .

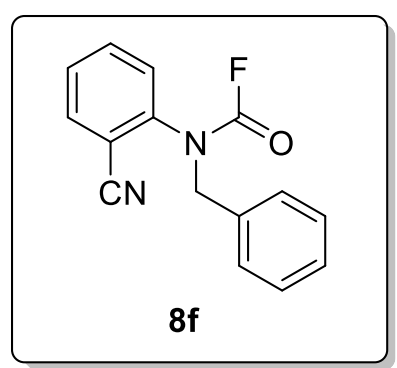

benzyl(2-cyanophenyl)carbamic fluoride (8f); $7 \mathbf{f}(76 \mathrm{mg})$ was used to provide 8f (48 mg, 63\%) as brown oil; $\mathrm{R}_{f}=0.4(\mathrm{Hex}: \mathrm{EtOAc}=3: 1) ;{ }^{1} \mathrm{H}$ $\operatorname{NMR}\left(400 \mathrm{MHz}, \mathrm{CDCl}_{3}\right.$, mixture of isomers, ratio 1:3) $\delta 7.70(\mathrm{~m}, 1 \mathrm{H}), 7.54$ (m, 1H), 7.43 (td, $J=7.6,1.2 \mathrm{~Hz}, 1 \mathrm{H}), 7.33-7.28(\mathrm{~m}, 3 \mathrm{H}), 7.23-7.15$ (m, 2.25), $7.04(\mathrm{~d}, J=7.9 \mathrm{~Hz}, 0.75 \mathrm{H}), 4.88(\mathrm{~s}, 2 \mathrm{H}) .{ }^{19} \mathrm{~F} \mathrm{NMR}\left(376 \mathrm{MHz}, \mathrm{CDCl}_{3}\right)$ $\delta-12.44(\mathrm{~s}, 0.75 \mathrm{~F}),-18.52(\mathrm{~s}, 0.25 \mathrm{~F}) .{ }^{13} \mathrm{C} \mathrm{NMR}\left(100 \mathrm{MHz}, \mathrm{CDCl}_{3}\right) \delta 146.1$ $\left(\mathrm{d}, J_{\mathrm{C}, \mathrm{F}}=292 \mathrm{~Hz}\right), 145.7\left(\mathrm{~d}, J_{\mathrm{C}, \mathrm{F}}=288 \mathrm{~Hz}\right), 142.2\left(\mathrm{~d}, J_{\mathrm{C}, \mathrm{F}}=1.2 \mathrm{~Hz}\right), 141.1$ $\left(\mathrm{d}, J_{\mathrm{C}, \mathrm{F}}=1.8 \mathrm{~Hz}\right), 134.6,134.4,134.0,133.8,129.4,129.3,129.2(2 \mathrm{C})$, $129.1,129.09,129.06,128.9(2 \mathrm{C}), 128.7(2 \mathrm{C}), 128.5,115.7,115.5,112.6,112.5,55.4\left(\mathrm{~d}, J_{\mathrm{C}, \mathrm{F}}=2.1 \mathrm{~Hz}\right)$, $55.3\left(\mathrm{~d}, J_{\mathrm{C}, \mathrm{F}}=2.1 \mathrm{~Hz}\right)$. IR (neat) $v_{\max } 3089,3037,2229,1789,1577,1189 \mathrm{~cm}^{-1} \mathrm{HRMS}[\mathrm{EI}+]$ calcd for $\mathrm{C}_{15} \mathrm{H}_{11} \mathrm{FN}_{2} \mathrm{O}[\mathrm{M}]^{+} 254.0855$, found 254.0856.

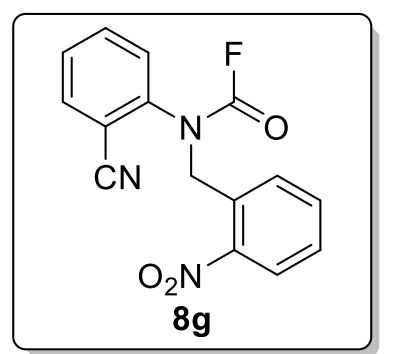

(2-cyanophenyl)(2-nitrobenzyl)carbamic fluoride (8g); $7 \mathrm{~g}(89 \mathrm{mg}$ ) was used to provide $\mathbf{8 g}(55 \mathrm{mg}, 61 \%)$ as yellow solid; $\mathrm{R}_{f}=0.5(\mathrm{Hex}:$ EtOAc $=$ 2:1); ${ }^{1} \mathrm{H} \mathrm{NMR}\left(400 \mathrm{MHz}, \mathrm{CDCl}_{3}\right.$, mixture of isomers, ratio 1:3) $\delta 8.03(\mathrm{~d}, J$ $=8.1 \mathrm{~Hz}, 0.25 \mathrm{H}), 7.95(\mathrm{~d}, J=8.1 \mathrm{~Hz}, 0.75 \mathrm{H}), 7.81(\mathrm{~d}, J=7.7 \mathrm{~Hz}, 1 \mathrm{H}), 7.72-$ $7.66(\mathrm{~m}, 2 \mathrm{H}), 7.60(\mathrm{td}, J=7.8,1.6 \mathrm{~Hz}, 1 \mathrm{H}), 7.54-7.44(\mathrm{~m}, 2 \mathrm{H}), 7.29(\mathrm{~d}, J=$ $8.1 \mathrm{~Hz}, 0.25 \mathrm{H}), 7.22(\mathrm{~d}, J=8.0 \mathrm{~Hz}, 0.75 \mathrm{H}), 5.35(\mathrm{~s}, 2 \mathrm{H}) .{ }^{19} \mathrm{~F}$ NMR $(376$ $\left.\mathrm{MHz}, \mathrm{CDCl}_{3}\right) \delta-12.07$ (s, 0.75F), -16.95 (s, 0.25F). ${ }^{13} \mathrm{C} \mathrm{NMR}(100 \mathrm{MHz}$, $\left.\mathrm{CDCl}_{3}\right) \delta 148.6(2 \mathrm{C}), 146.3\left(\mathrm{~d}, J_{\mathrm{C}, \mathrm{F}}=375 \mathrm{~Hz}\right), 145.7\left(\mathrm{~d}, J_{\mathrm{C}, \mathrm{F}}=289 \mathrm{~Hz}\right), 142.1$ $\left(\mathrm{d}, J_{\mathrm{C}, \mathrm{F}}=1.3 \mathrm{~Hz}\right), 141.2\left(\mathrm{~d}, J_{\mathrm{C}, \mathrm{F}}=1.7 \mathrm{~Hz}\right), 134.33,134.30,134.2,134.1(2 \mathrm{C}), 133.9,131.8,130.6,130.0$, $129.8,129.5,129.4(2 \mathrm{C}), 129.2,128.4,128.3,125.6,125.2,115.8,115.5,112.4(2 \mathrm{C}), 52.0\left(\mathrm{~d}, J_{\mathrm{C}, \mathrm{F}}=2.7\right.$ $\mathrm{Hz}), 51.8\left(\mathrm{~d}, J_{\mathrm{C}, \mathrm{F}}=1.0 \mathrm{~Hz}\right)$. IR (neat) $v_{\max } 2229,1783,1596,1518,1274 \mathrm{~cm}^{-1}$. HRMS[FAB+] calcd for $\mathrm{C}_{15} \mathrm{H}_{11} \mathrm{FN}_{3} \mathrm{O}_{3}[\mathrm{M}]^{+} 300.0784$, found 300.0785 .

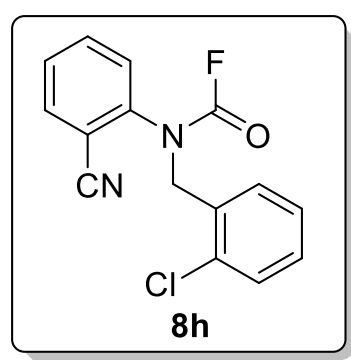

(2-chlorobenzyl)(2-cyanophenyl)carbamic fluoride (8h); 7h (86 mg) was used to provide $\mathbf{8 h}(55 \mathrm{mg}, 64 \%)$ as white solid; $\mathrm{R}_{f}=0.4(\mathrm{Hex}: \mathrm{EtOAc}=5: 1)$; ${ }^{1} \mathrm{H}$ NMR $\left(400 \mathrm{MHz}, \mathrm{CDCl}_{3}\right.$, mixture of isomers, ratio 1: 3) $\delta 7.67(\mathrm{~m}, 1 \mathrm{H})$, $7.52(\mathrm{td}, J=7.7,1.6 \mathrm{~Hz}, 1 \mathrm{H}), 7.43(\mathrm{~m}, 1.8 \mathrm{H}), 7.30(\mathrm{~m}, 1.2 \mathrm{H}), 7.24-7.19(\mathrm{~m}$, $2 \mathrm{H}), 7.20(\mathrm{~m}, 0.25 \mathrm{H}), 7.12(\mathrm{~d}, J=7.9 \mathrm{~Hz}, 0.75 \mathrm{H}), 5.11-5.08(\mathrm{~s}, 2 \mathrm{H}) .{ }^{19} \mathrm{~F}$ NMR $\left(376 \mathrm{MHz}, \mathrm{CDCl}_{3}\right) \delta-12.32$ (s, 0.75F), $-18.33(\mathrm{~s}, 0.25 \mathrm{~F}) .{ }^{13} \mathrm{C} \mathrm{NMR}(100 \mathrm{MHz}$, $\left.\mathrm{CDCl}_{3}\right) \delta 146.1\left(\mathrm{~d}, J_{\mathrm{C}, \mathrm{F}}=293 \mathrm{~Hz}\right), 145.5\left(\mathrm{~d}, J_{\mathrm{C}, \mathrm{F}}=288 \mathrm{~Hz}\right), 141.8\left(\mathrm{~d}, J_{\mathrm{C}, \mathrm{F}}=1.0\right.$ $\mathrm{Hz}), 140.8\left(\mathrm{~d}, J_{\mathrm{C}, \mathrm{F}}=1.7 \mathrm{~Hz}\right), 134.4,134.1,133.99,133.91,133.8,133.7,132.0$, $131.8(2 \mathrm{C}), 130.8,130.2(2 \mathrm{C}), 130.1,129.8,129.3(2 \mathrm{C}), 129.2,129.1,127.5,127.4,115.8,115.6,112.6$, $112.5,52.2\left(\mathrm{~d}, J_{\mathrm{C}, \mathrm{F}}=2.6 \mathrm{~Hz}\right), 52.1\left(\mathrm{~d}, J_{\mathrm{C}, \mathrm{F}}=2.1 \mathrm{~Hz}\right) . \mathrm{IR}$ (neat) $v_{\max } 2231,1791,1594,1480,1452,1384$, $1255 \mathrm{~cm}^{-1}$. HRMS[EI+] calcd for $\mathrm{C}_{15} \mathrm{H}_{10} \mathrm{ClFN}_{2} \mathrm{O}[\mathrm{M}]^{+}$288.0466, found 288.0446.

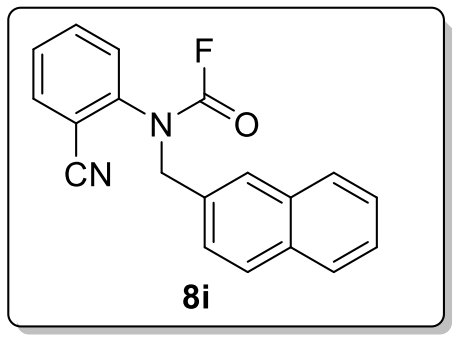
(m, 2H). $\left.{ }^{19} \mathrm{~F} \mathrm{NMR} \mathrm{(376} \mathrm{MHz,} \mathrm{CDCl}_{3}\right) \delta-11.65$ (s, 0.8F), -18.37 (s, 0.2F). ${ }^{13} \mathrm{C}$ NMR (100 MHz, $\left.\mathrm{CDCl}_{3}\right)$

(2-cyanophenyl)(naphthalen-2-ylmethyl)carbamic fluoride (8i); $7 \mathbf{i}$ $\left(91 \mathrm{mg}\right.$ ) was used to provide $\mathbf{8 i}(57 \mathrm{mg}, 63 \%)$ as off-white solid; $\mathrm{R}_{f}=$ 0.6 (Hex:EtOAc $=2: 1)$; ${ }^{1} \mathrm{H}$ NMR $\left(400 \mathrm{MHz}, \mathrm{CDCl}_{3}\right.$, mixture of isomers, ratio 1:4) $\delta 8.08(\mathrm{~d}, J=8.3 \mathrm{~Hz}, 0.8 \mathrm{H}), 7.89(\mathrm{~s}, 0.2 \mathrm{H}), 7.86(\mathrm{~d}$, $J=7.3 \mathrm{~Hz}, 1 \mathrm{H}), 7.81(\mathrm{~s}, 0.2 \mathrm{H}), 7.78(\mathrm{~d}, J=8.0 \mathrm{~Hz}, 0.8 \mathrm{H}), 7.65(\mathrm{~m}$, $0.2 \mathrm{H}), 7.62(\mathrm{dd}, J=6.7,2.0 \mathrm{~Hz}, 0.8 \mathrm{H}), 7.59-7.50(\mathrm{~m}, 2 \mathrm{H}), 7.37-7.29$ $(\mathrm{m}, 2.4 \mathrm{H}), 7.25(\mathrm{~d}, J=15.1 \mathrm{~Hz}, 0.6 \mathrm{H}), 7.15(\mathrm{~d}, J=6.9 \mathrm{~Hz}, 0.8 \mathrm{H}), 6.92$ $(\mathrm{m}, 0.2 \mathrm{H}), 6.73(\mathrm{~m}, 0.2 \mathrm{H}), 6.66(\mathrm{dd}, J=7.5,1.1 \mathrm{~Hz}, 0.8 \mathrm{H}), 5.79-5.13$ 
$\delta 147.2\left(\mathrm{~d}, J_{\mathrm{C}, \mathrm{F}}=497 \mathrm{~Hz}\right), 145.5\left(\mathrm{~d}, J_{\mathrm{C}, \mathrm{F}}=288 \mathrm{~Hz}\right), 141.8\left(\mathrm{~d}, J_{\mathrm{C}, \mathrm{F}}=1.2 \mathrm{~Hz}\right), 140.4\left(\mathrm{~d}, J_{\mathrm{C}, \mathrm{F}}=1.5 \mathrm{~Hz}\right)$, $134.1,133.9,133.8,133.7,133.6,133.5,132.4,131.6,131.2,129.84,129.82,129.7,129.6,129.5$, $129.17,129.14,129.0(2 \mathrm{C}), 127.8,127.3,127.1,126.3,125.2,125.0,123.4,122.6,118.1,115.8,115.5$, $115.2,112.4(2 \mathrm{C}), 52.6\left(\mathrm{~d}, J_{\mathrm{C}, \mathrm{F}}=2.5 \mathrm{~Hz}\right), 52.4\left(\mathrm{~d}, J_{\mathrm{C}, \mathrm{F}}=2.9 \mathrm{~Hz}\right.$ ). IR (neat) $v_{\max } 2228,1775,1488,1455$, $1378,1251 \mathrm{~cm}^{-1}$. HRMS[EI+] calcd for $\mathrm{C}_{19} \mathrm{H}_{13} \mathrm{FN}_{2} \mathrm{O}[\mathrm{M}]^{+} 304.1012$, found 304.1011.

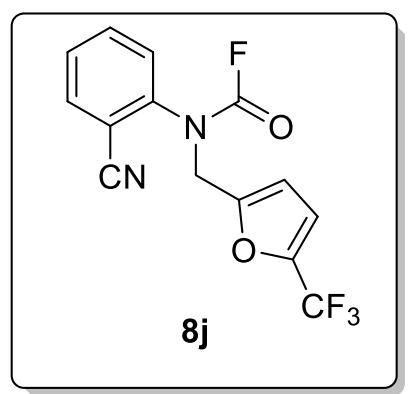

(2-cyanophenyl)((5-(trifluoromethyl)furan-2-yl)methyl)carbamic fluoride (8j); $\mathbf{7 j}$ (93 $\mathrm{mg}$ ) was used to provide $\mathbf{8 j}$ (67 $\mathrm{mg}, 72 \%$ ) as yellow oil; $\mathrm{R}_{f}=0.5$ (Hex:EtOAc $\left.=2: 1\right) ;{ }^{1} \mathrm{H}$ NMR $\left(400 \mathrm{MHz}, \mathrm{CDCl}_{3}\right.$, mixture of isomers, ratio 1: 4) $\delta 7.74(\mathrm{~m}, 1 \mathrm{H}), 7.65(\mathrm{~m}, 1 \mathrm{H}), 7.51(\mathrm{td}, J=7.7,1.2 \mathrm{~Hz}$, $1 \mathrm{H}), 7.32(\mathrm{~d}, J=8.0 \mathrm{~Hz}, 0.2 \mathrm{H}), 7.25(\mathrm{~d}, J=7.5 \mathrm{~Hz}, 0.8 \mathrm{H}), 6.73(\mathrm{~m}, 1 \mathrm{H})$, $6.46(\mathrm{~d}, J=3.2 \mathrm{~Hz}, 0.8 \mathrm{H}), 6.40(\mathrm{~d}, J=3.4 \mathrm{~Hz}, 0.2 \mathrm{H}), 5.02-4.69(\mathrm{~m}, 2 \mathrm{H})$ ${ }^{19} \mathrm{~F}$ NMR $\left(376 \mathrm{MHz}, \mathrm{CDCl}_{3}\right) \delta-12.39(\mathrm{~s}, 0.75 \mathrm{~F}),-17.66(\mathrm{~s}, 0.25 \mathrm{~F}) .{ }^{19} \mathrm{~F}$ NMR (376 MHz, $\left.\mathrm{CDCl}_{3}\right) \delta-12.39$ (s, 0.8F), -17.66 (s, 0.2F), 64.37-64.38 $(\mathrm{m}, 3 \mathrm{~F}) .{ }^{13} \mathrm{C} \mathrm{NMR}\left(100 \mathrm{MHz}, \mathrm{CDCl}_{3}\right) \delta 150.8(2 \mathrm{C}), 150.7\left(\mathrm{~d}, J_{\mathrm{C}, \mathrm{F}}=1.8 \mathrm{~Hz}\right)$, $145.6\left(\mathrm{~d}, J_{\mathrm{C}, \mathrm{F}}=293 \mathrm{~Hz}\right), 145.1\left(\mathrm{~d}, J_{\mathrm{C}, \mathrm{F}}=289 \mathrm{~Hz}\right), 142.4\left(\mathrm{q}, J_{\mathrm{C}, \mathrm{F}}=422 \mathrm{~Hz}\right), 141.8,140.8\left(\mathrm{~d}, J_{\mathrm{C}, \mathrm{F}}=1.8\right.$ Hz), 134.4, 134.3, 133.9, 133.8, 129.7, 129.6, 129.2, 129.1, 118.8 (q, $\left.J_{\mathrm{C}, \mathrm{F}}=265 \mathrm{~Hz}\right), 115.2,114.8,112.7$ $\left(\mathrm{q}, J_{\mathrm{C}, \mathrm{F}}=2.8 \mathrm{~Hz}\right), 112.6(2 \mathrm{C}), 112.5(2 \mathrm{C}), 111.5,111.0,47.58,47.56$. IR (neat) $v_{\max } 3141,2233,1803$, $1598,1562,1319 \mathrm{~cm}^{-1}$. HRMS[EI+] calcd for $\mathrm{C}_{14} \mathrm{H}_{8} \mathrm{~F}_{4} \mathrm{~N}_{2} \mathrm{O}_{2}[\mathrm{M}]^{+} 312.0522$, found 312.0535 .

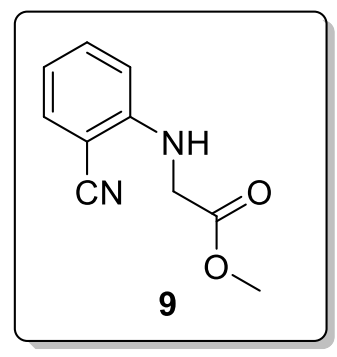

Methyl (2-cyanophenyl)glycinate (9); $7 \mathbf{k}$ (70 $\mathrm{mg}$ ) was used to provide $\mathbf{9}$ (25 $\mathrm{mg}, 44 \%)$ as white solid; $\mathrm{R}_{f}=0.4$ (Hex:EtOAc $\left.=3: 1\right) ;{ }^{1} \mathrm{H}$ NMR $(300 \mathrm{MHz}$, $\left.\mathrm{CDCl}_{3}\right) 7.45-7.37(\mathrm{~m}, 2 \mathrm{H}), 6.75(\mathrm{td}, J=7.5,0.9 \mathrm{~Hz}, 1 \mathrm{H}), 6.48(\mathrm{~d}, J=8.4 \mathrm{~Hz}$, $1 \mathrm{H}), 5.13$ (br s, 1H), $4.00(\mathrm{~d}, J=5.5 \mathrm{~Hz}, 2 \mathrm{H}), 3.80(\mathrm{~s}, 3 \mathrm{H})$. The data is in agreement with the literature. ${ }^{7}$

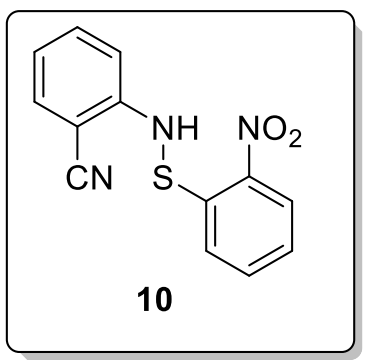

2-(((2-nitrophenyl)thio)amino)benzonitrile (10); 71 (95 $\mathrm{mg})$ was used to provide $10(16 \mathrm{mg}, 20 \%)$ as yellow solid; $\mathrm{R}_{f}=0.7(\mathrm{Hex}:$ EtOAc $=2: 1) ;{ }^{1} \mathrm{H}$ $\operatorname{NMR}\left(300 \mathrm{MHz}, \mathrm{CDCl}_{3}\right) \delta 8.35(\mathrm{dd}, J=8.3,1.4 \mathrm{~Hz}, 1 \mathrm{H}), 7.63-7.48(\mathrm{~m}, 3 \mathrm{H})$, $7.44(\mathrm{td}, J=7.2,1.6 \mathrm{~Hz}, 1 \mathrm{H}), 7.35(\mathrm{td}, J=7.0,1.4 \mathrm{~Hz}, 1 \mathrm{H}), 7.22(\mathrm{~d}, J=8.4$ $\mathrm{Hz}, 1 \mathrm{H}), 6.96(\mathrm{td}, J=7.5,1.0 \mathrm{~Hz}, 1 \mathrm{H}), 5.98(\mathrm{~s}, 1 \mathrm{H}) .{ }^{13} \mathrm{C}$ NMR $(100 \mathrm{MHz}$, $\left.\mathrm{CDCl}_{3}\right) \delta 148.7,143.2,142.4,134.79,134.73,132.8,126.2,126.0,123.8$, $121.0,116.9,114.1,99.0$. IR (neat) $v_{\max } 3273,2225,1591,1569,1485,1451$, $1332,1285 \mathrm{~cm}^{-1}$. HRMS[EI+] calcd for $\mathrm{C}_{14} \mathrm{H}_{8} \mathrm{~F}_{4} \mathrm{~N}_{2} \mathrm{O}_{2}[\mathrm{M}]^{+} 271.0415$ found 271.0418 .

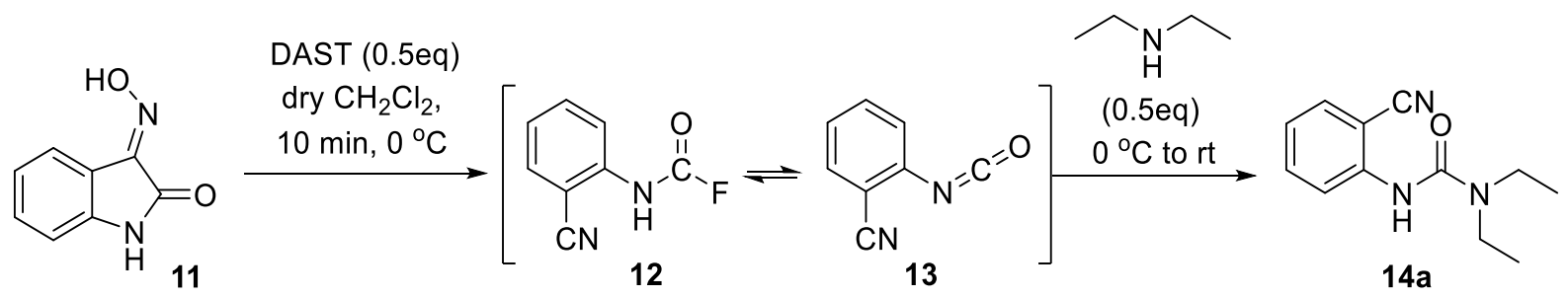

\footnotetext{
7 Romagnoli, R.; Prencipe, F.; Oliva, P.; Salvador, M.K.; Brancale, A.; Ferla, S.; Hamel, E.; Viola, G.; Bortolozzi, R.; Persoons, L.; Balzarini, J.; Liekens, S.; Schols, D. Design, Synthesis and Biological Evaluation of 2Alkoxycarbonyl-3-Anilinoindoles as a New Class of Potent Inhibitors of Tubulin Polymerization. Bioorg. Chem. 2020, $97,103665$.
} 
To a stirred solution of 3-(hydroxyimino)indolin-2-one $11(162 \mathrm{mg}, 1.0 \mathrm{mmol})$ in dry $\mathrm{CH}_{2} \mathrm{Cl}_{2}(3.0 \mathrm{~mL})$ was slowly added diethylaminosulfur trifluoride (DAST, $66 \mu \mathrm{L}, 0.5 \mathrm{mmol}$ ) at $0{ }^{\circ} \mathrm{C}$ under Ar. After 10 minutes, diethylamine $(52 \mu \mathrm{L}, 0.5 \mathrm{mmol})$ was slowly added to the reaction mixture at $0{ }^{\circ} \mathrm{C}$. After being stirred for 30 minutes at $\mathrm{rt}$, the reaction mixture was diluted with sat. $\mathrm{NH}_{4} \mathrm{Cl}$ aq. sol'n $(5.0 \mathrm{~mL})$ and extracted with EtOAc $(10 \times 3 \mathrm{~mL})$. The organic solution was dried over $\mathrm{MgSO}_{4}$, filtered, and concentrated. The crude mixture was subjected to silica gel column chromatography (EtOAc:Hex =1:7 to $1: 3)$ to provide $14 a(210 \mathrm{mg}, 97 \%)$ as a yellow oil. $\mathrm{R}_{f}=0.4(\mathrm{Hex}: \mathrm{EtOAc}=1: 3) ;{ }^{1} \mathrm{H} \mathrm{NMR}(400 \mathrm{MHz}$, DMSO- $\left.d_{6}\right) \delta 8.48(\mathrm{~s}, 1 \mathrm{H}), 7.71(\mathrm{~d}, J=7.7 \mathrm{~Hz}, 1 \mathrm{H}), 7.60(\mathrm{t}, J=8.1 \mathrm{~Hz}, 1 \mathrm{H}), 7.45(\mathrm{~d}, J=8.1 \mathrm{~Hz}, 1 \mathrm{H})$, $7.23(\mathrm{t}, J=7.2 \mathrm{~Hz}, 1 \mathrm{H}), 3.36-3.33(\mathrm{~m}, 4 \mathrm{H}), 1.13(\mathrm{t}, J=7.0 \mathrm{~Hz}, 6 \mathrm{H})$. The spectral data is in agreement with the known literature values. ${ }^{8}$

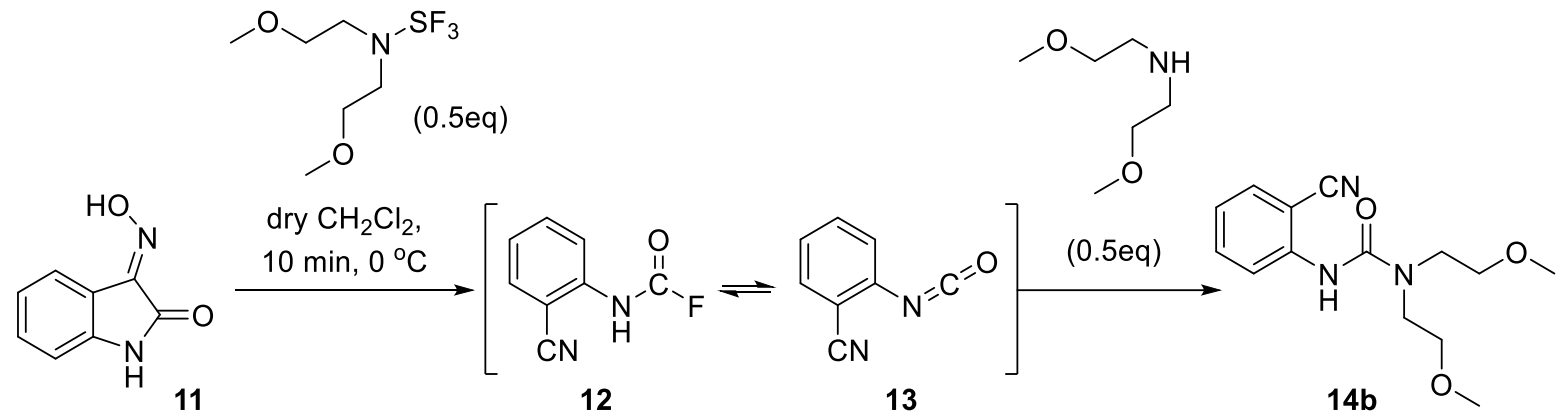

To a stirred solution of 3-(hydroxyimino)indolin-2-one 11 (162 mg, $1.0 \mathrm{mmol})$ in dry $\mathrm{CH}_{2} \mathrm{Cl}_{2}(3.0 \mathrm{~mL})$ was slowly added bis(2-methoxyethyl)aminosulfur trifluoride (Deoxo-Fluor, $90 \mu \mathrm{L}, 0.5 \mathrm{mmol}$ ) at $0{ }^{\circ} \mathrm{C}$ under Ar. After 10 minutes, bis(2-methoxyethyl)amine $(74 \mu \mathrm{L}, 0.5 \mathrm{mmol})$ was slowly added to the reaction mixture at $0{ }^{\circ} \mathrm{C}$. After being stirred for 1 hour at $\mathrm{rt}$, the mixture was diluted with sat. $\mathrm{NH}_{4} \mathrm{Cl}$ aq. sol'n $(5.0 \mathrm{~mL})$ and extracted with EtOAc $(3 \mathrm{X} 10 \mathrm{~mL})$. The organic solution was dried over $\mathrm{MgSO}_{4}$, filtered, and concentrated. The crude mixture was subjected to silica gel column chromatography (EtOAc:Hex $=1: 5$ to $1: 1)$ to provide $\mathbf{1 4 b}(226 \mathrm{mg}, 82 \%)$ as a yellow solid. $\mathrm{R}_{f}=0.4(\mathrm{Hex}: \mathrm{EtOAc}=1: 1)$; ${ }^{1} \mathrm{H}$ NMR $\left(400 \mathrm{MHz}, \mathrm{CDCl}_{3}\right) \delta 8.75(\mathrm{~s}, 1 \mathrm{H}), 7.96(\mathrm{~d}, J=4.3 \mathrm{~Hz}, 1 \mathrm{H}), 7.49-7.43(\mathrm{~m}, 2 \mathrm{H}), 6.97(\mathrm{t}, J=7.6$ $\mathrm{Hz}, 1 \mathrm{H}), 3.62-3.57(\mathrm{~m}, 8 \mathrm{H}), 3.42(\mathrm{~s}, 6 \mathrm{H}) .{ }^{13} \mathrm{C} \mathrm{NMR}\left(100 \mathrm{MHz}, \mathrm{CDCl}_{3}\right)$ 156.4, 143.3, 133.5, 132.7, 121.8 , 120.9, 117.0, 101.3, 71.9, 59.0, 49.2. IR (neat) $v_{\max } 3295,2872,2217,1662,1530,1448,1109 \mathrm{~cm}^{-1}$. HRMS[EI+] calcd for $\mathrm{C}_{14} \mathrm{H}_{19} \mathrm{~N}_{3} \mathrm{O}_{3}[\mathrm{M}]^{+} 277.1420$, found 277.1426 .
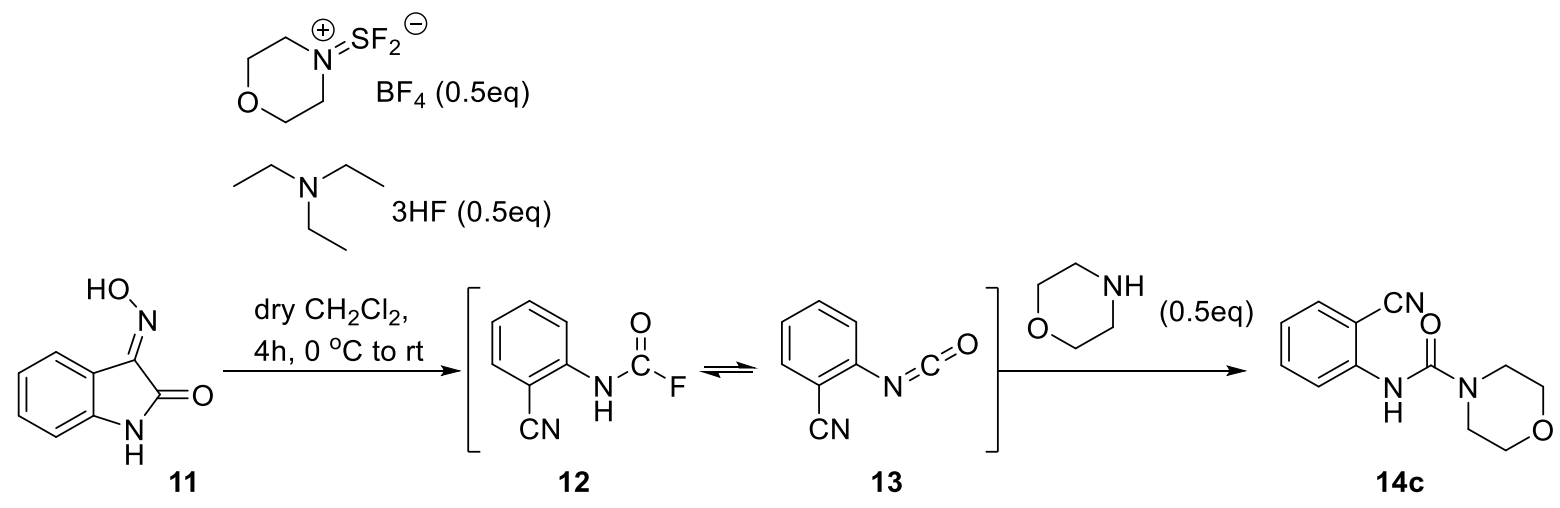

To a stirred solution of triethylamine trihydrofluoride $(80 \mu \mathrm{L}, 0.5 \mathrm{mmol})$ in dry $\mathrm{CH}_{2} \mathrm{Cl}_{2}(3.0 \mathrm{~mL})$ was added 3-(hydroxyimino)indolin-2-one $11(162 \mathrm{mg}, 1.0 \mathrm{mmol})$ and difluoro(morpholino)sulfonium tetrafluoroborate (XtalFluor-M, $114 \mathrm{mg}, 0.5 \mathrm{mmol}$ ) at $0{ }^{\circ} \mathrm{C}$ under Ar. After stirring for 4 hours at rt, morpholine ( $43 \mu \mathrm{L}, 0.5 \mathrm{mmol}$ ) was slowly added to the reaction mixture at $0{ }^{\circ} \mathrm{C}$. After 2 hours at $\mathrm{rt}$, the

\footnotetext{
${ }^{8}$ Holzschneider, K.; Mohr, F.; Kirsch, S. F. Synthesis and Reactivity of 3,3-Diazidooxindoles. Org. Lett. 2018, 20,7066 .
} 
mixture was diluted with sat. $\mathrm{NH}_{4} \mathrm{Cl}$ aq. sol'n $(5.0 \mathrm{~mL})$ and extracted with EtOAc $(3 \mathrm{X} 10 \mathrm{~mL})$. The organic solution was dried over $\mathrm{MgSO}_{4}$, filtered, and concentrated. The crude mixture was subjected to silica gel column chromatography ( $1 \%$ to $2 \% \mathrm{MeOH}$ in DCM) to provide $14 \mathrm{c}$ [118 $\mathrm{mg}, 51 \%(71 \%$ brsm)] as a yellow solid. $\mathrm{R}_{f}=0.4\left(3 \% \mathrm{MeOH}\right.$ in DCM); ${ }^{1} \mathrm{H}$ NMR $\left(400 \mathrm{MHz}\right.$, DMSO- $\left.d_{6}\right) \delta 8.88(\mathrm{~s}, 1 \mathrm{H})$, $7.74(\mathrm{~d}, J=6.9 \mathrm{~Hz}, 1 \mathrm{H}), 7.62(\mathrm{t}, J=7.4 \mathrm{~Hz}, 1 \mathrm{H}), 7.42(\mathrm{dd}, J=8.0,1.5 \mathrm{~Hz}, 1 \mathrm{H}), 7.26(\mathrm{td}, J=7.5,1.9$ $\mathrm{Hz}, 1 \mathrm{H}), 3.63-3.60(\mathrm{~m}, 4 \mathrm{H}), 3.46-3.43(\mathrm{~m}, 4 \mathrm{H})$. The spectral data is in agreement with the known literature values. ${ }^{8}$<smiles>N#CCCCN(Cc1ccccc1)C(=O)F</smiles>

6b

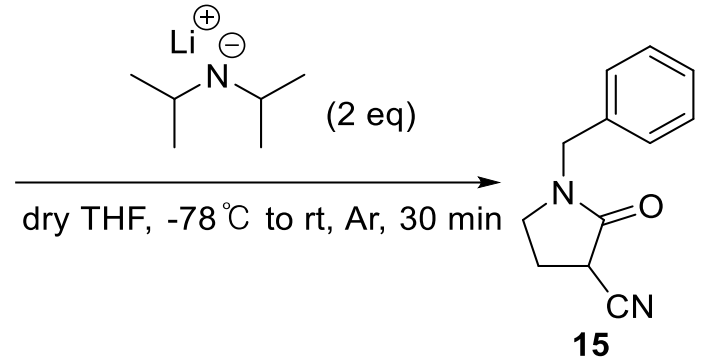

15

Lithium diisopropylamide $(2.0 \mathrm{M}$ in THF, $1.0 \mathrm{~mL}, 2.0 \mathrm{mmol})$ was added to a stirred solution of benzyl(3-cyanopropyl)carbamic fluoride $6 \mathbf{b}(220 \mathrm{mg}, 1.0 \mathrm{mmol})$ in dry THF $(3.0 \mathrm{~mL})$ at $-78^{\circ} \mathrm{C}$ under Ar. After 30 minutes, the reaction mixture warmed to $\mathrm{rt}$ and quenched with sat. $\mathrm{NH}_{4} \mathrm{Cl}$ aq. sol'n $(10 \mathrm{~mL})$ and extracted with $\mathrm{CH}_{2} \mathrm{Cl}_{2}(3 \mathrm{X} 10 \mathrm{~mL})$. The organic solution was dried over $\mathrm{MgSO}_{4}$, filtered, and concentrated. The residue was subjected to silica gel column chromatography ( $1 \%$ to $2 \% \mathrm{MeOH}$ in $\mathrm{DCM})$ to afford 1-benzyl-2-oxopyrrolidine-3-carbonitrile $15(136 \mathrm{mg}, 68 \%)$ as pink oil. $\mathrm{R}_{f}=0.4(3 \%$ $\mathrm{MeOH}$ in DCM); ${ }^{1} \mathrm{H}$ NMR (300 MHz, $\left.\mathrm{CDCl}_{3}\right) \delta$ 7.37-7.21 (m, 5H), 4.52-4.41 (m, 2H), $3.56(\mathrm{t}, J=8.9$ $\mathrm{Hz}, 1 \mathrm{H}), 3.40-3.23(\mathrm{~m}, 2 \mathrm{H}), 2.50-2.17(\mathrm{~m}, 2 \mathrm{H}) .{ }^{13} \mathrm{C} \mathrm{NMR}\left(100 \mathrm{MHz}, \mathrm{CDCl}_{3}\right)$ 166.0, 135.2, 129.0, 128.3, 128.2, 117.1, 47.5, 44.7, 33.7, 23.7. IR (neat) $v_{\max } 2924,2249,1704,1435,1283 \mathrm{~cm}^{-1}$. HRMS[EI+] calcd for $\mathrm{C}_{14} \mathrm{H}_{19} \mathrm{~N}_{3} \mathrm{O}_{3}[\mathrm{M}]^{+} 200.0950$, found 200.0957 .

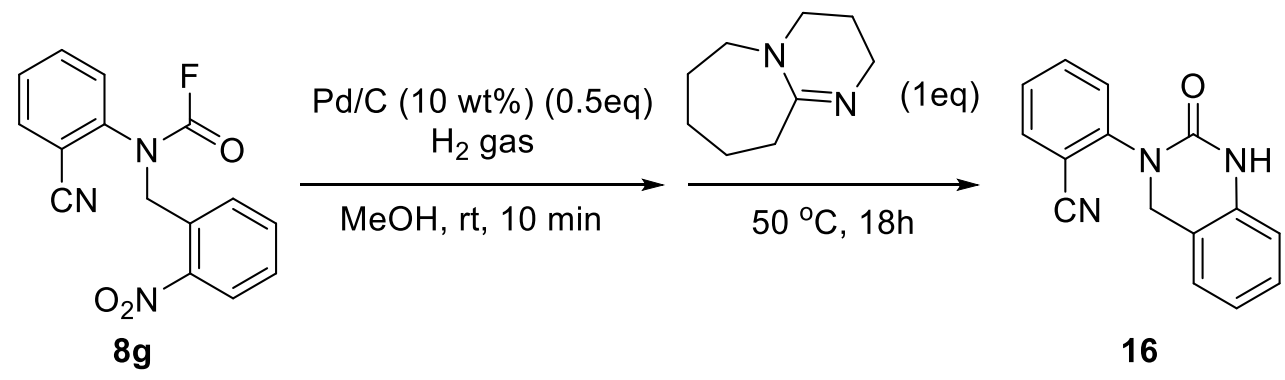

A two-neck $25 \mathrm{~mL}$ round bottom flask was charged with carbamoyl fluoride $8 \mathrm{~g}$ (300 $\mathrm{mg}, 1.0 \mathrm{mmol})$, $10 \%$ palladium on carbon $(52 \mathrm{mg}, 0.05 \mathrm{mmol})$, and $\mathrm{MeOH}(3.0 \mathrm{~mL})$. The reaction flask was flushed with a balloon filled with hydrogen gas for $5 \mathrm{~min}$ and further stirred under atmospheric pressure for 10 min at rt. Then, 1,8-diazabicyclo[5.4.0]undec-7-ene $(0.15 \mathrm{~mL}, 1.0 \mathrm{mmol})$ was added in and the reaction mixture was stirred for 18 hours at $50{ }^{\circ} \mathrm{C}$ in heating mantle. The reaction mixture was cooled to rt, filtered through a pad of Celite, and washed with ethyl acetate. The filtrate was then washed with water and brine. The organic solution was dried over $\mathrm{MgSO}_{4}$, filtered, and concentrated. The crude mixture was subjected to silica gel column chromatography (1\% to $2 \% \mathrm{MeOH}$ in DCM) to afford 2-(2-oxo-1,4dihydroquinazolin-3(2H)-yl)benzonitrile $16(170 \mathrm{mg}, 68 \%)$ as a white solid. $\mathrm{R}_{f}=0.5(3 \% \mathrm{MeOH}$ in DCM); ${ }^{1} \mathrm{H}$ NMR (300 MHz, $\left.\mathrm{CDCl}_{3}\right) \delta 8.33(\mathrm{br} \mathrm{s}, 1 \mathrm{H}), 7.75-7.65(\mathrm{~m}, 2 \mathrm{H}), 7.50(\mathrm{~d}, J=8.0 \mathrm{~Hz}, 1 \mathrm{H}), 7.42$ $(\mathrm{td}, J=7.6,1.5 \mathrm{~Hz}, 1 \mathrm{H}), 7.19(\mathrm{td}, J=7.5,1.5 \mathrm{~Hz}, 1 \mathrm{H}), 7.06(\mathrm{~d}, J=7.5 \mathrm{~Hz}, 1 \mathrm{H}), 6.97(\mathrm{td}, J=7.4,1.1$ $\mathrm{Hz}, 1 \mathrm{H}), 6.78(\mathrm{~d}, J=8.0 \mathrm{~Hz}, 1 \mathrm{H}), 4.86(\mathrm{~s}, 2 \mathrm{H}) .{ }^{13} \mathrm{C} \mathrm{NMR}\left(125 \mathrm{MHz}, \mathrm{CDCl}_{3}\right) 153.8,144.8,136.5,134.0$, $133.8,128.7,128.0,127.6,125.4,122.6,117.7,116.6,114.5,112.4,52.0$. IR (neat) $v_{\max } 2922,2229$, $1738,1666,1598,1487,1460 \mathrm{~cm}^{-1}$. HRMS[EI+] calcd for $\mathrm{C}_{14} \mathrm{H}_{19} \mathrm{~N}_{3} \mathrm{O}_{3}[\mathrm{M}]^{+}$249.0902, found 249.0901 . 


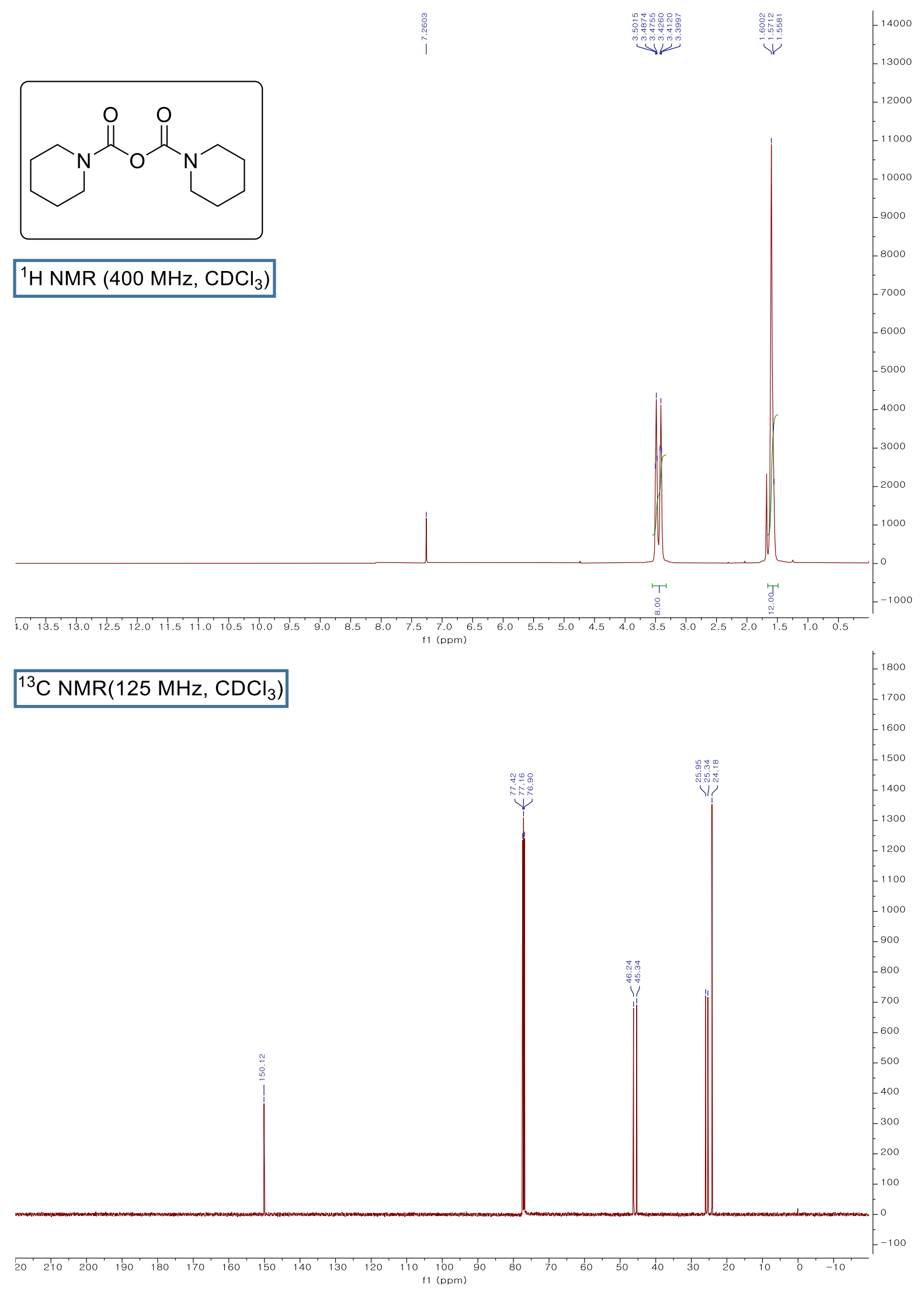




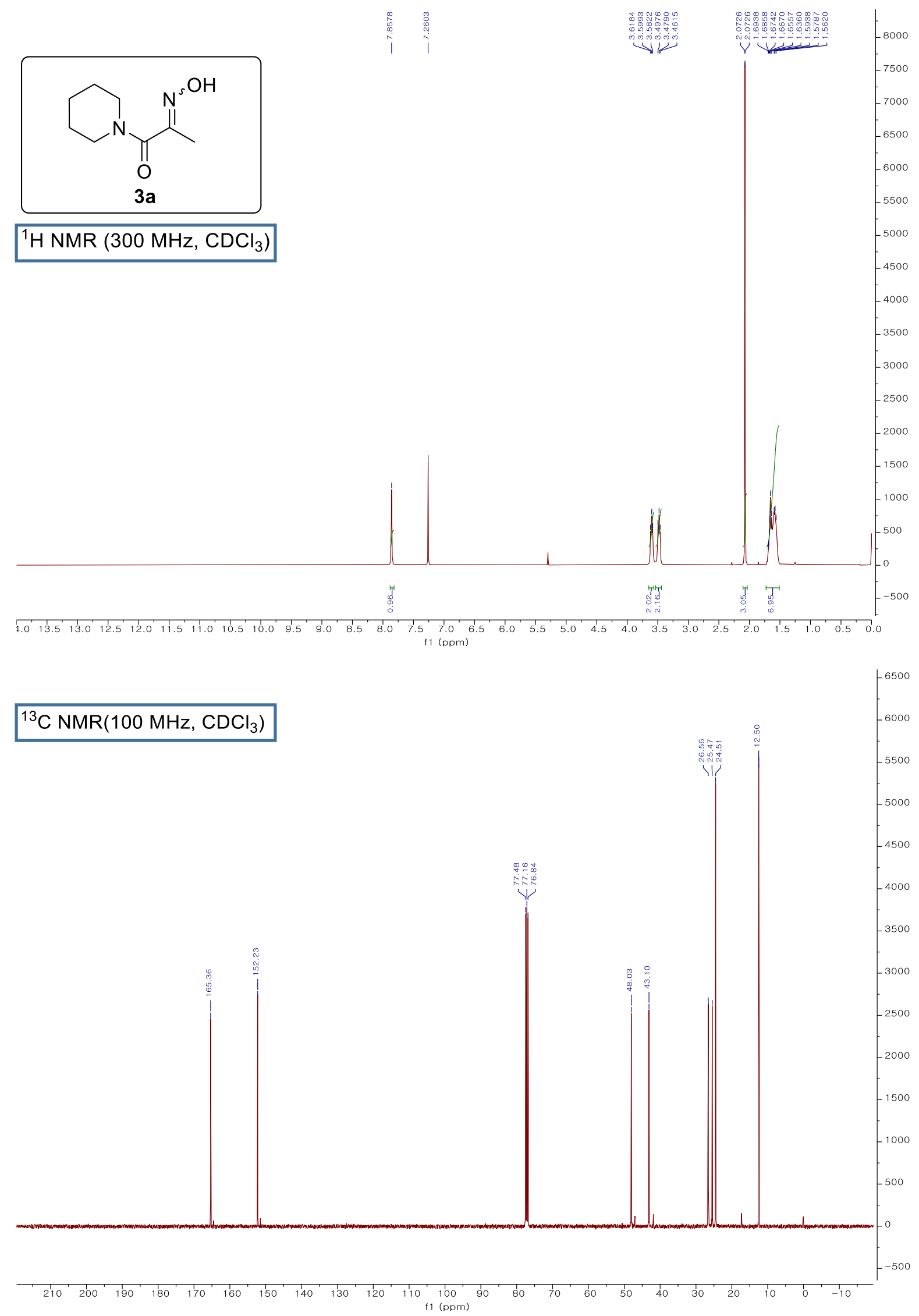



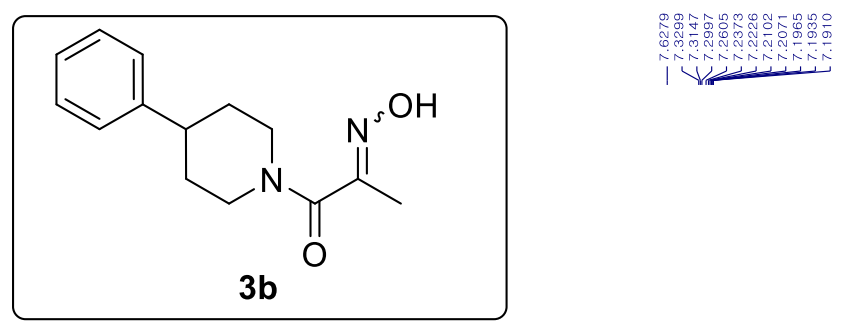

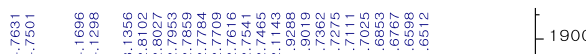

${ }^{1} \mathrm{H} \mathrm{NMR}\left(500 \mathrm{MHz}, \mathrm{CDCl}_{3}\right)$

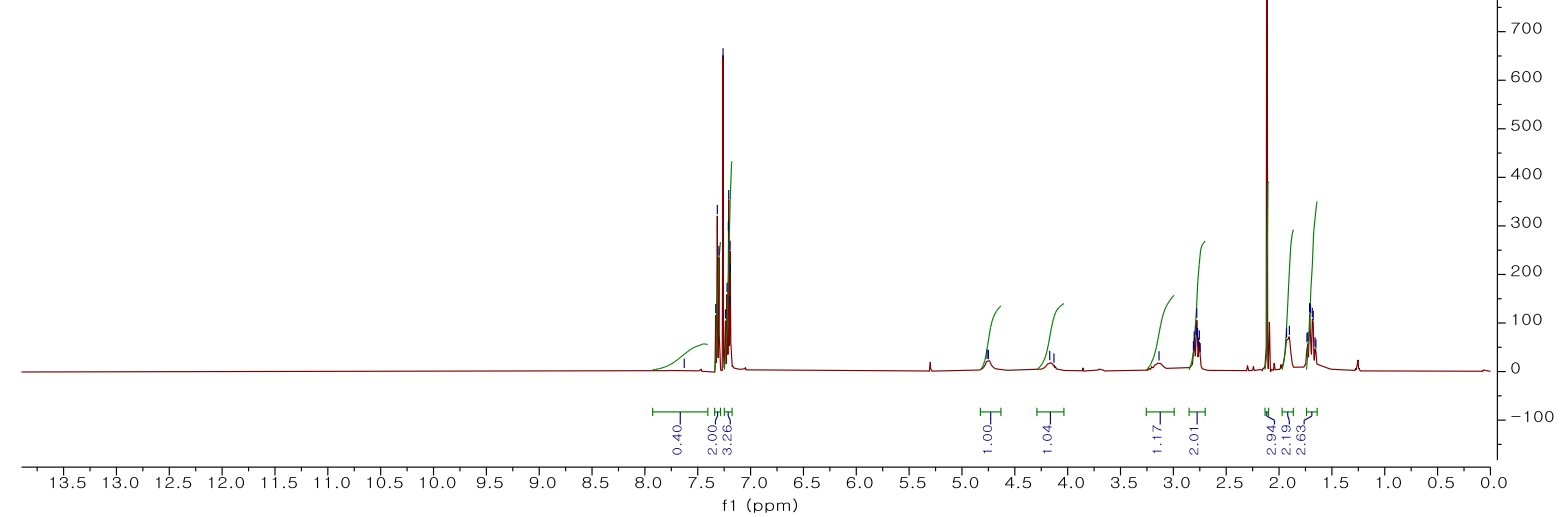

${ }^{13} \mathrm{C} \mathrm{NMR}\left(100 \mathrm{MHz}, \mathrm{CDCl}_{3}\right)$

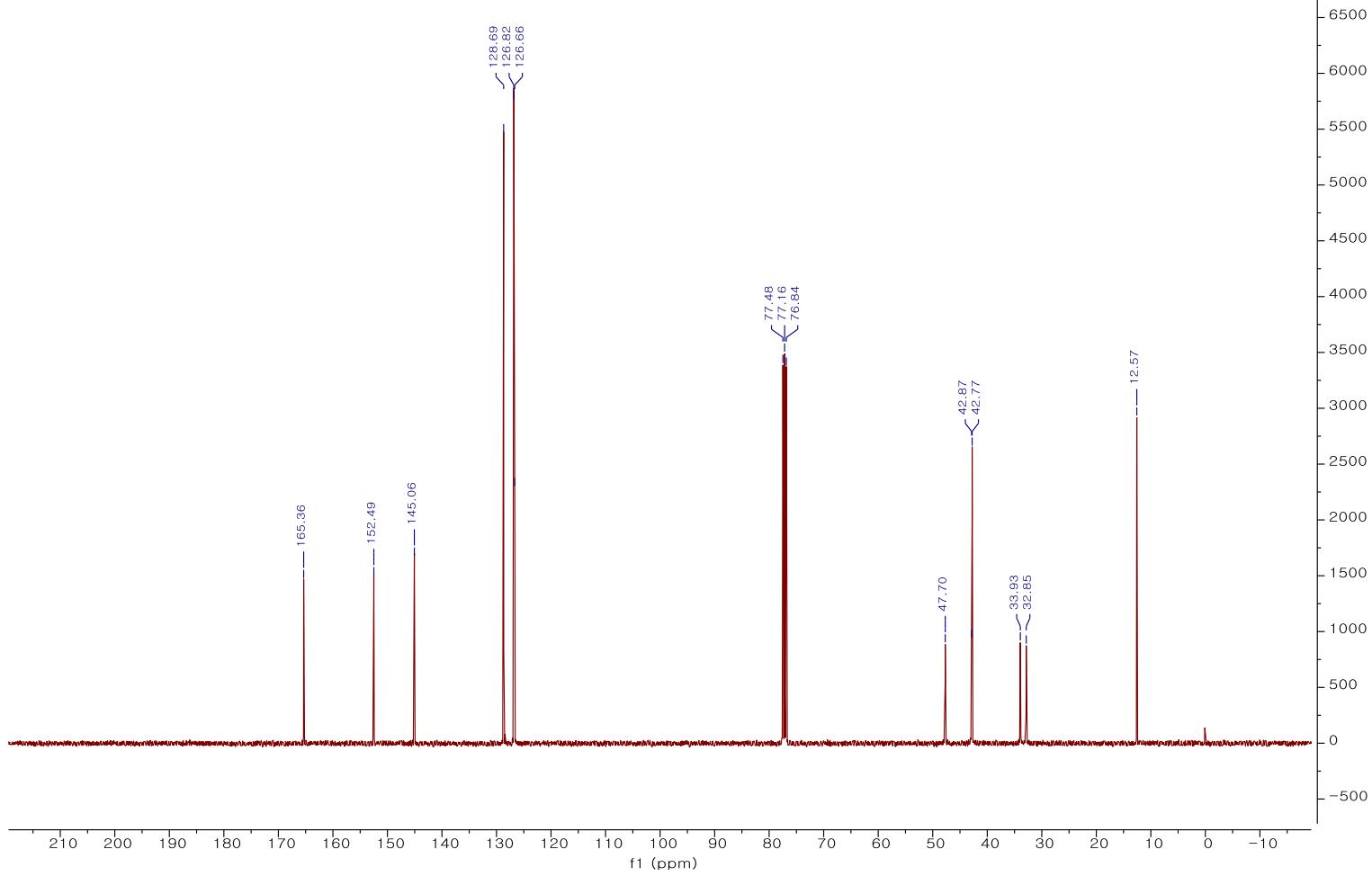




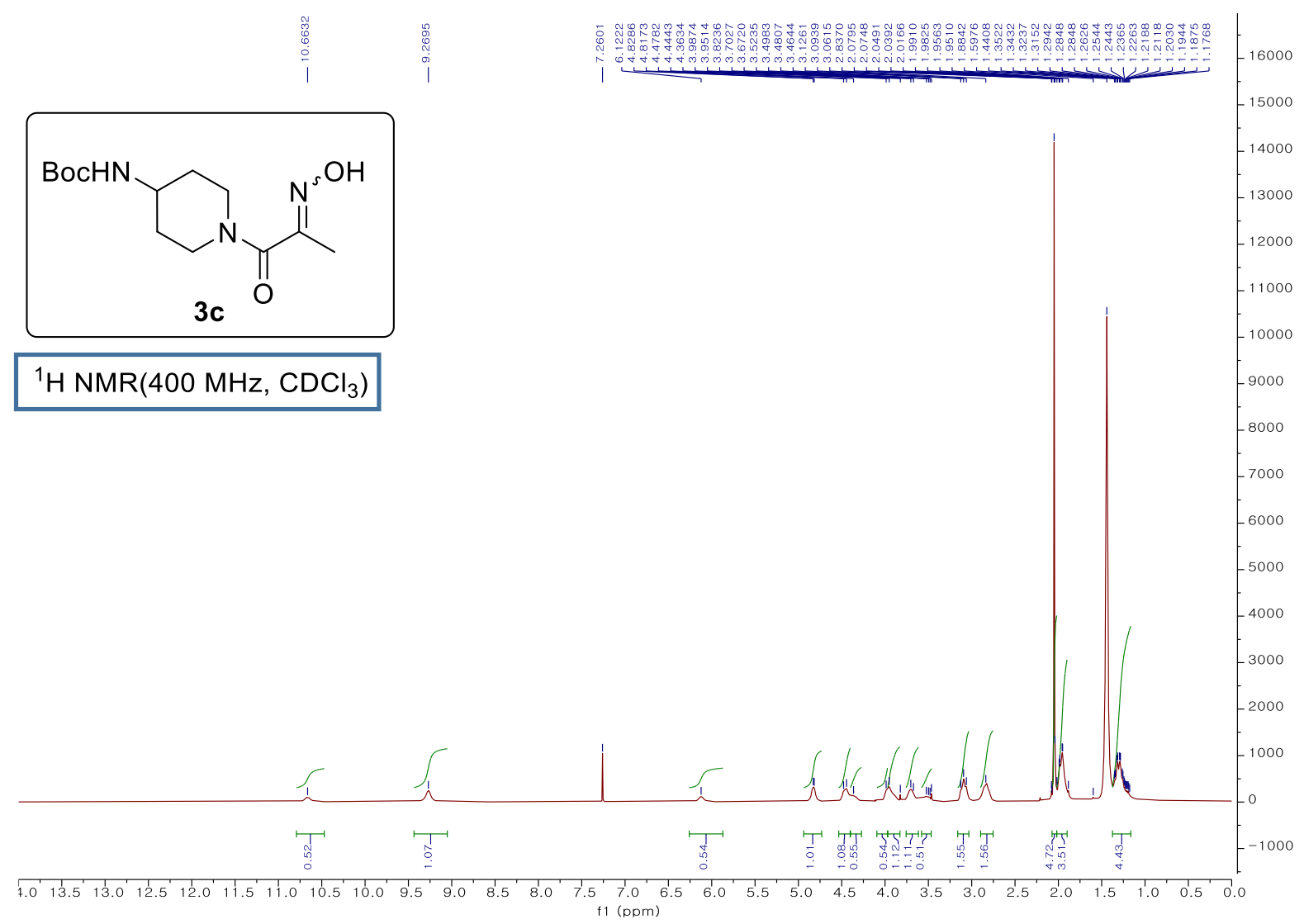

${ }^{13} \mathrm{C} \mathrm{NMR}\left(100 \mathrm{MHz}, \mathrm{CDCl}_{3}\right)$

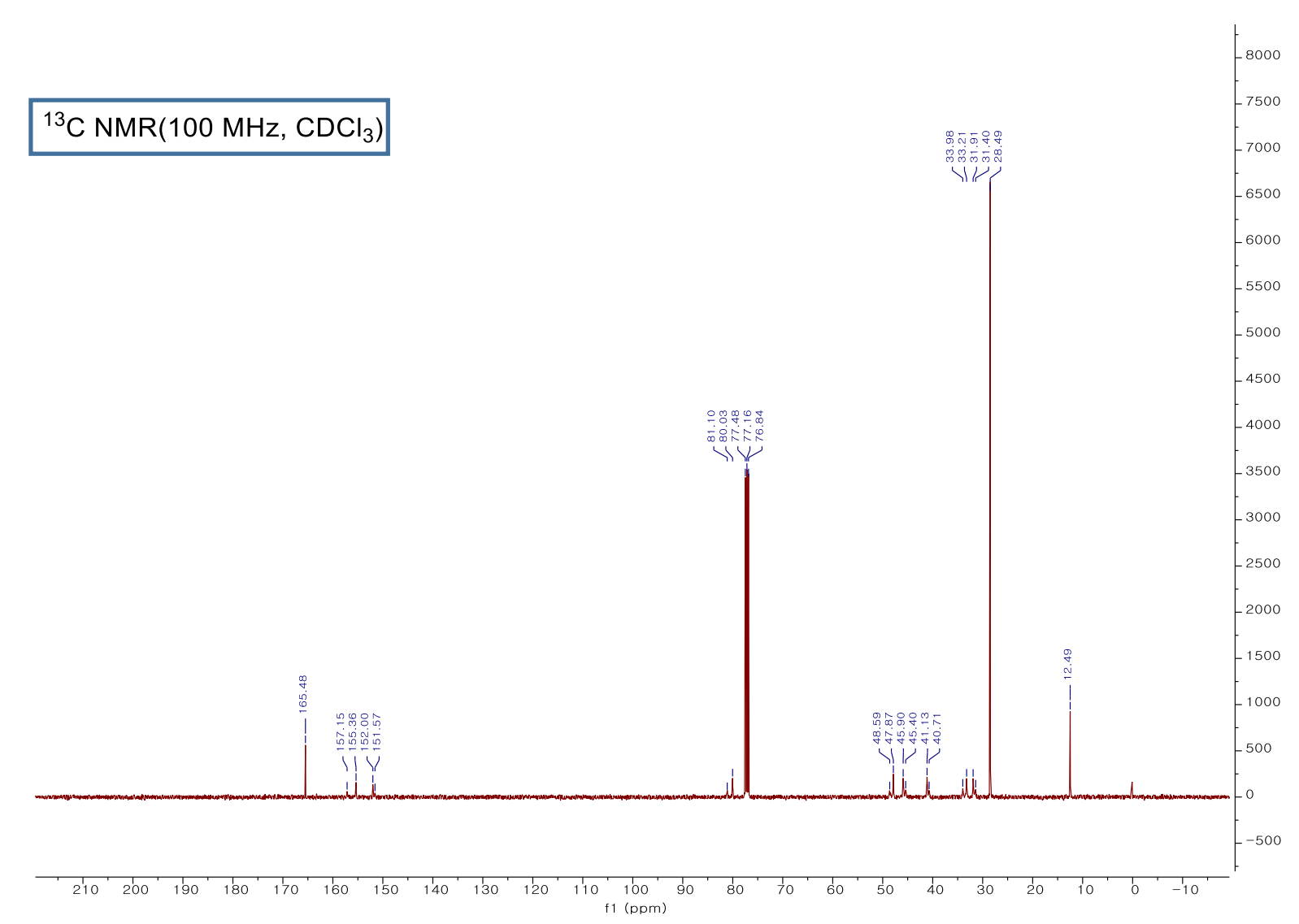



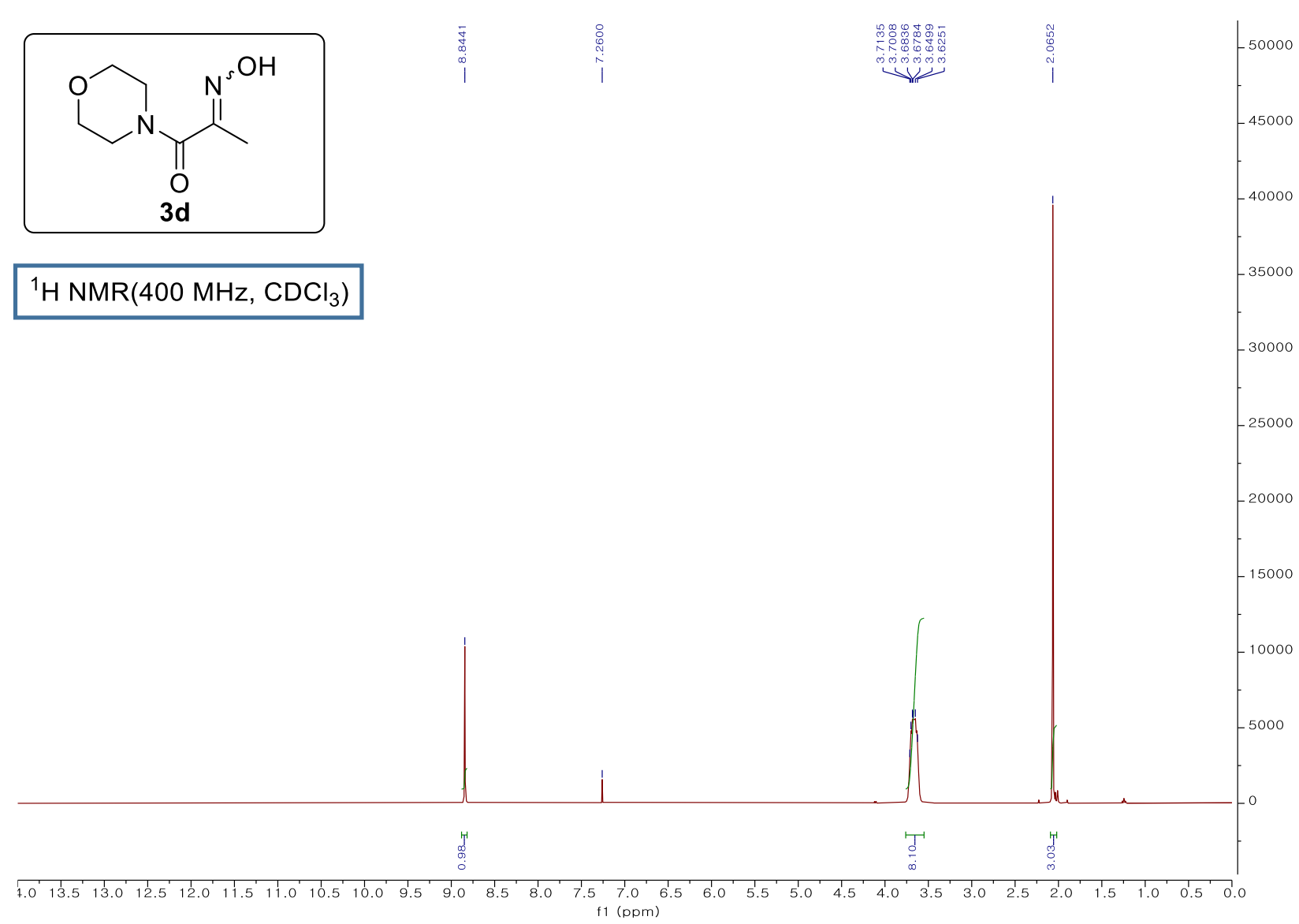

${ }^{13} \mathrm{C} \mathrm{NMR}\left(100 \mathrm{MHz}, \mathrm{CDCl}_{3}\right)$ f1 (ppm)

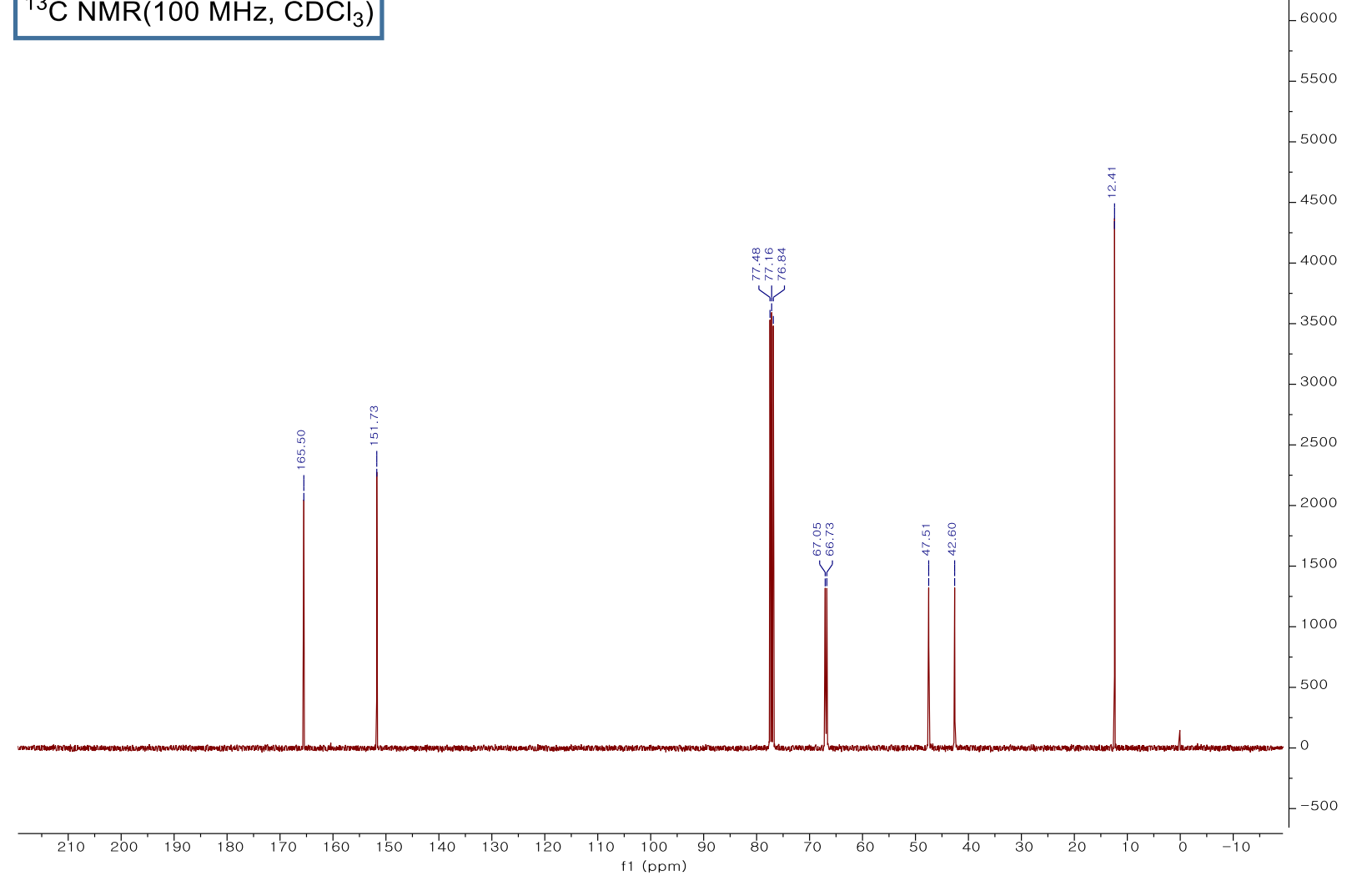




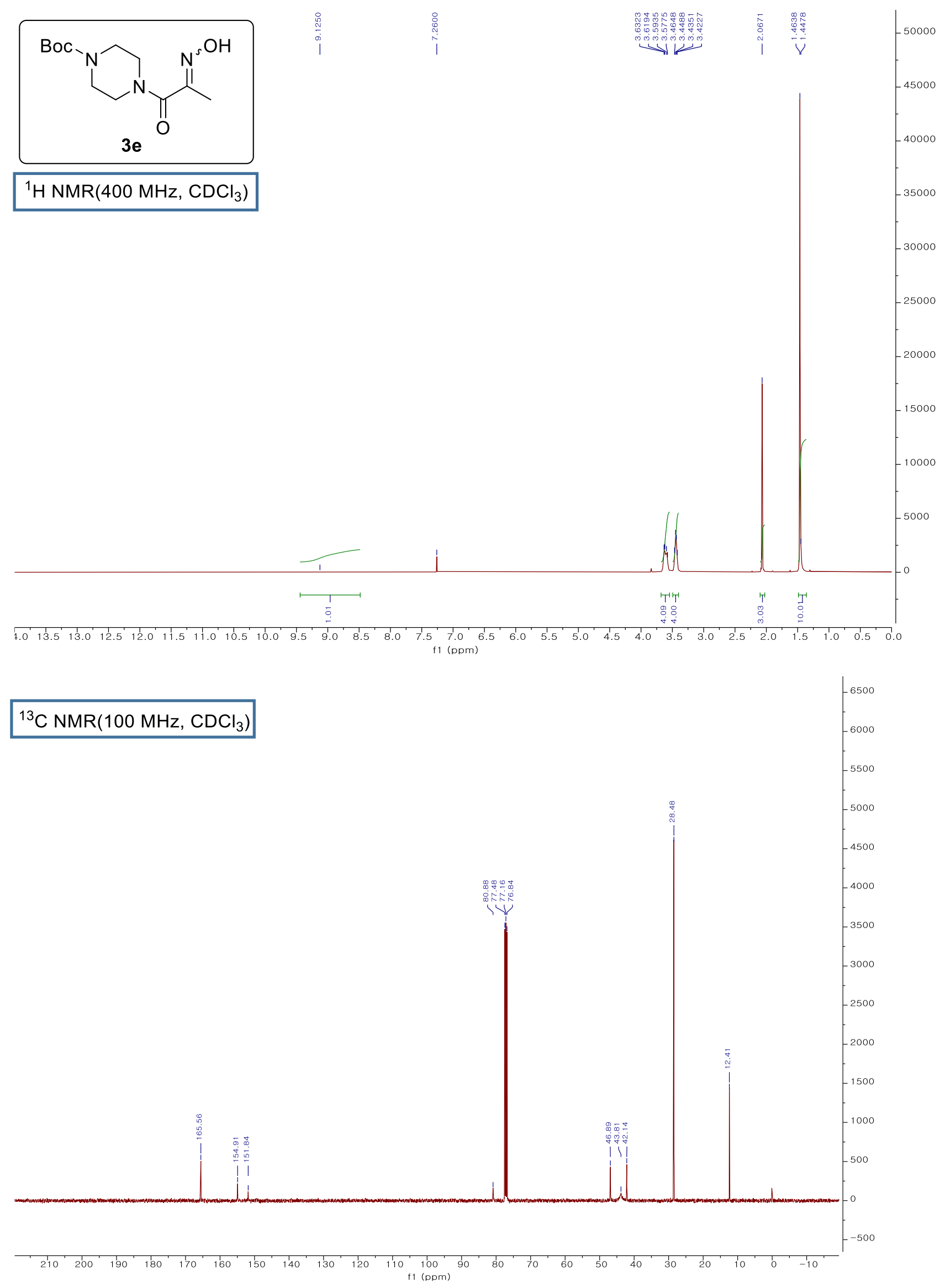




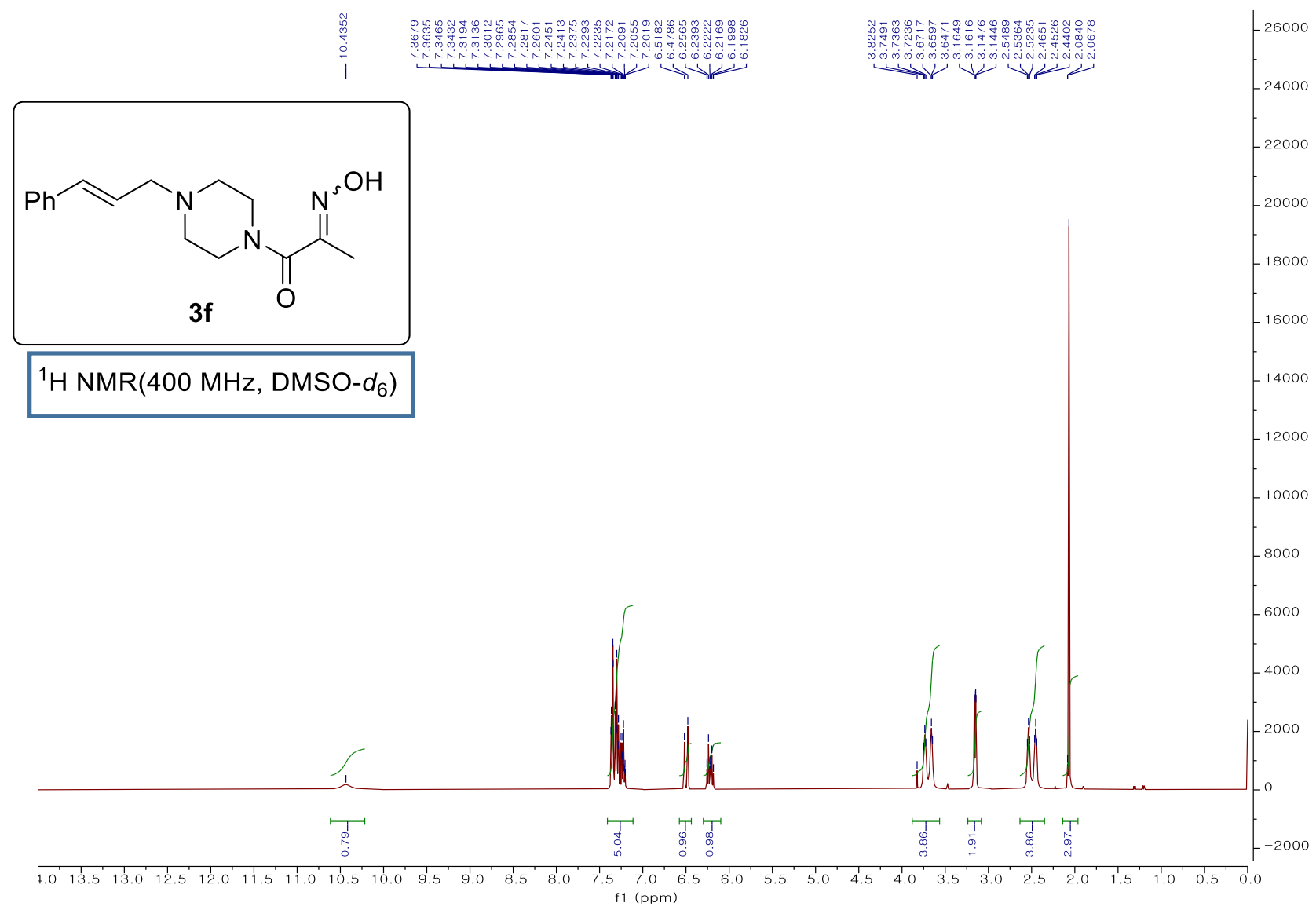

${ }^{13} \mathrm{C} \mathrm{NMR}\left(100 \mathrm{MHz}, \mathrm{CDCl}_{3}\right)$

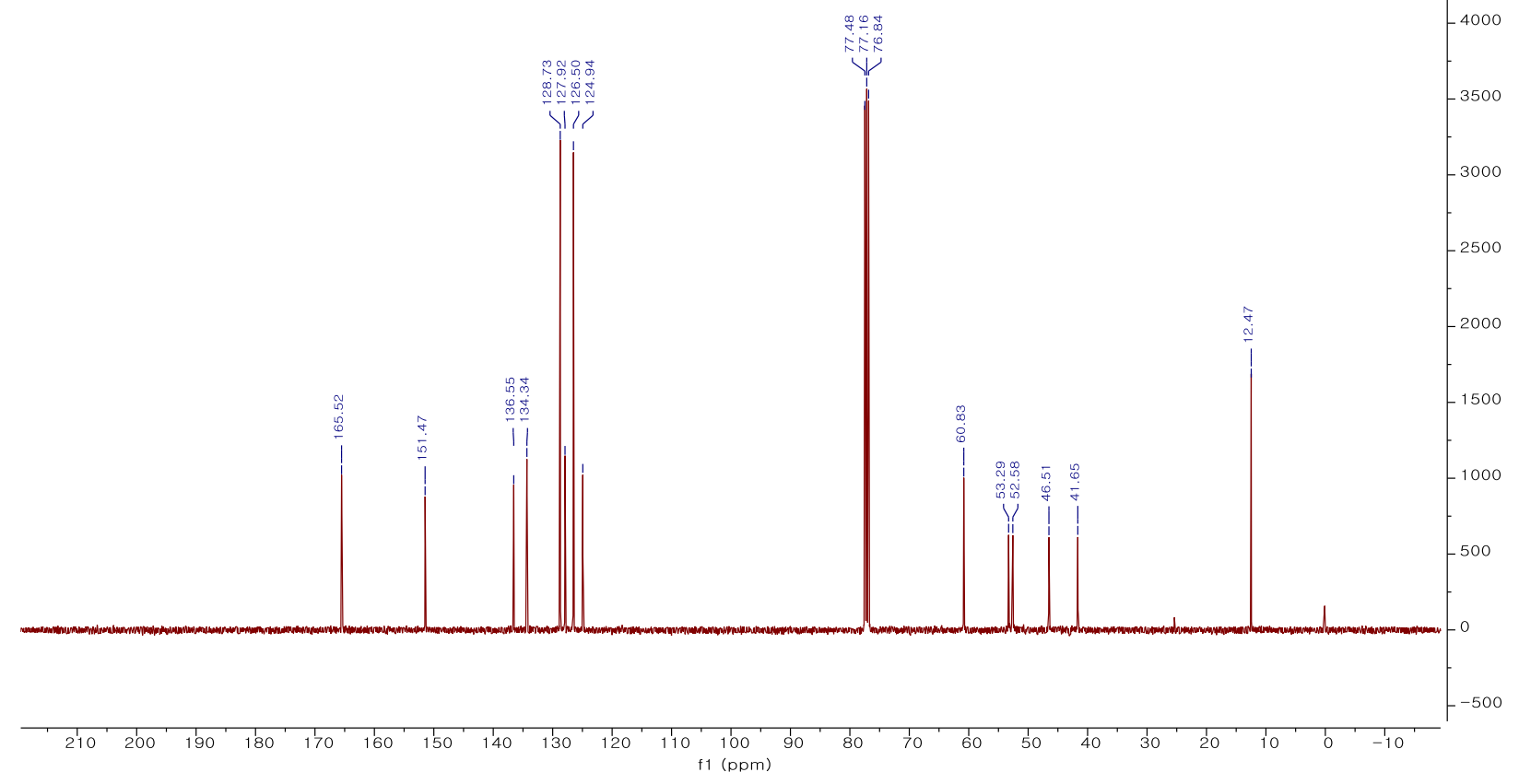




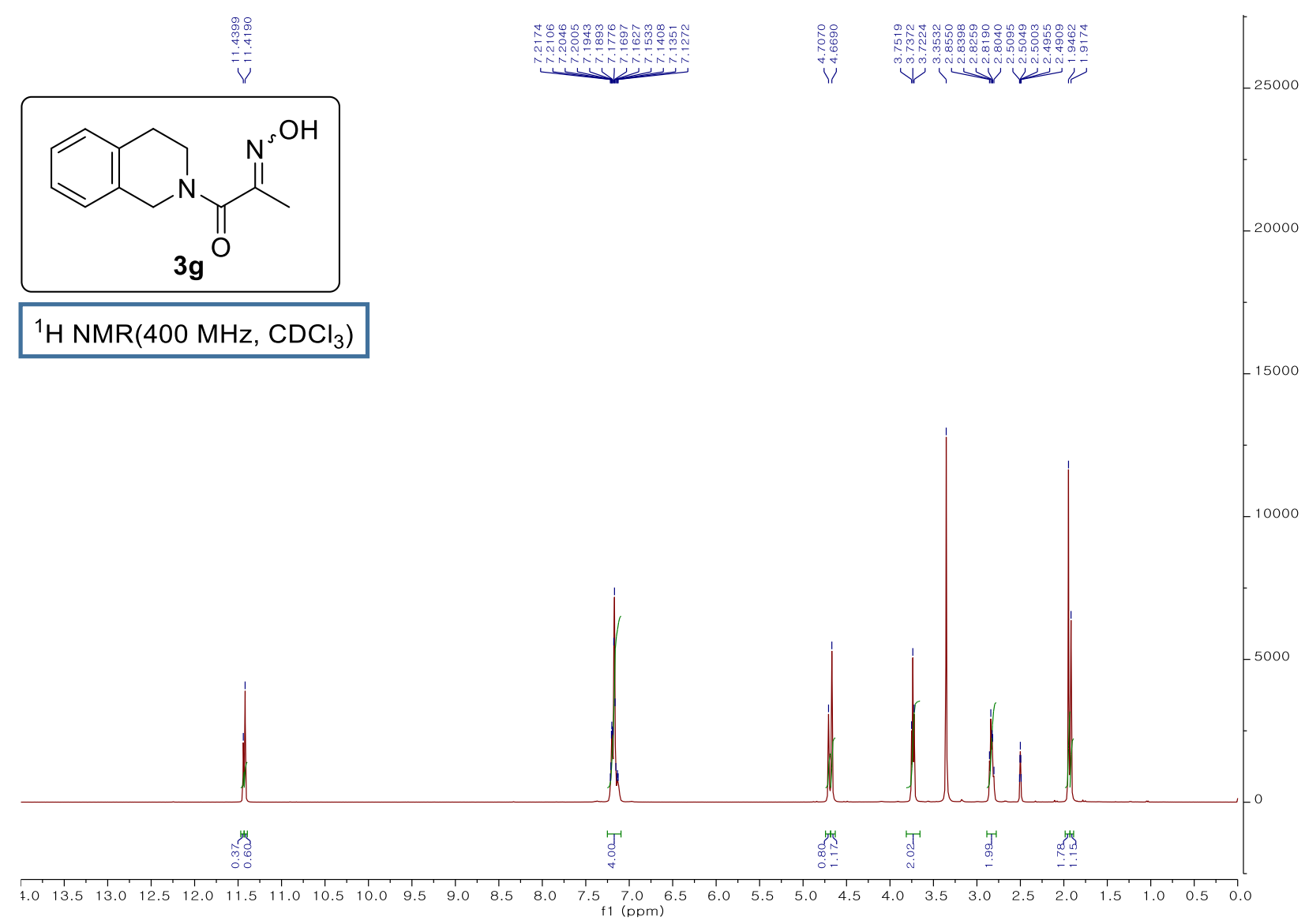

${ }^{13} \mathrm{C}$ NMR(100 MHz, DMSO- $\left.d_{6}\right)$

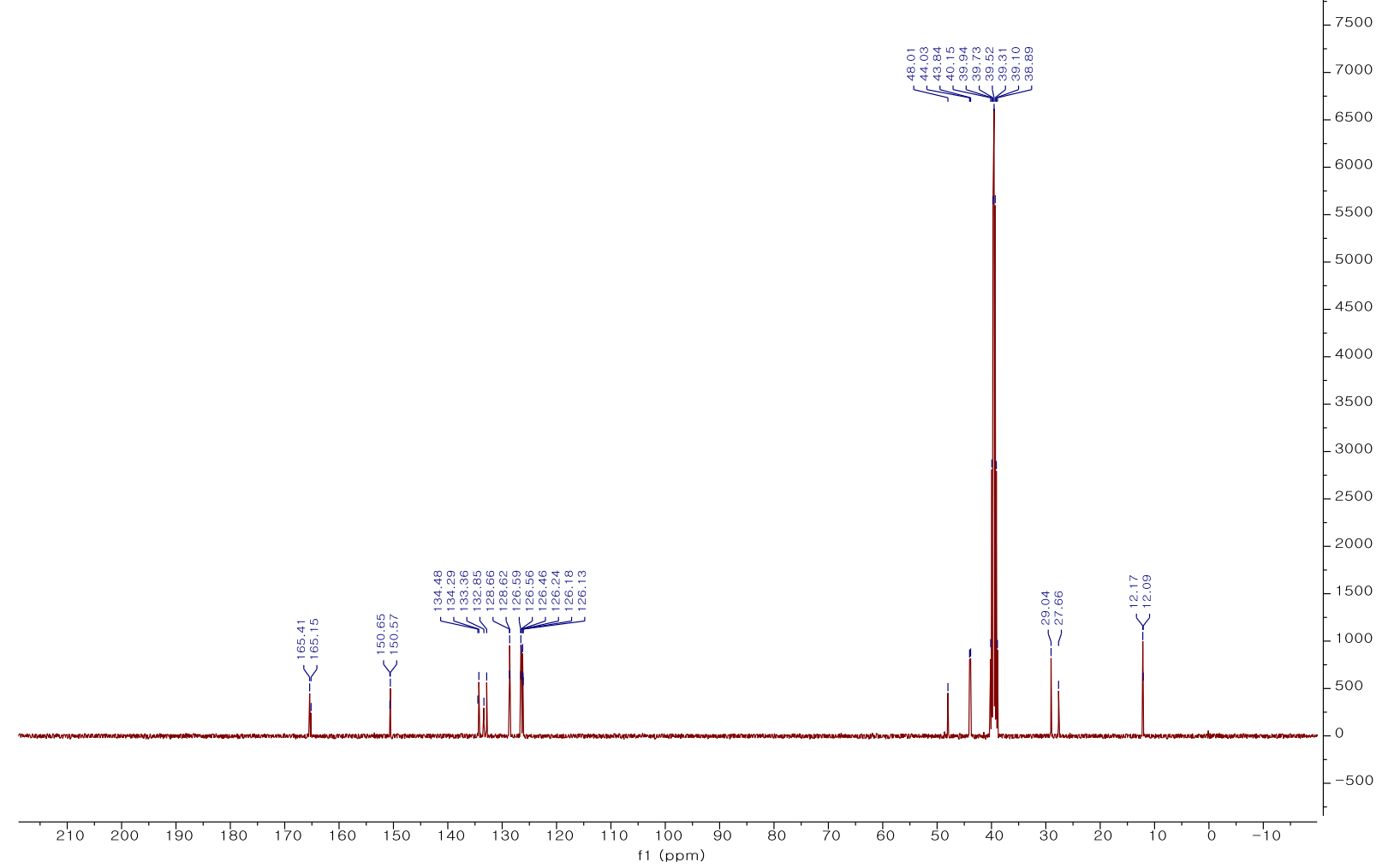



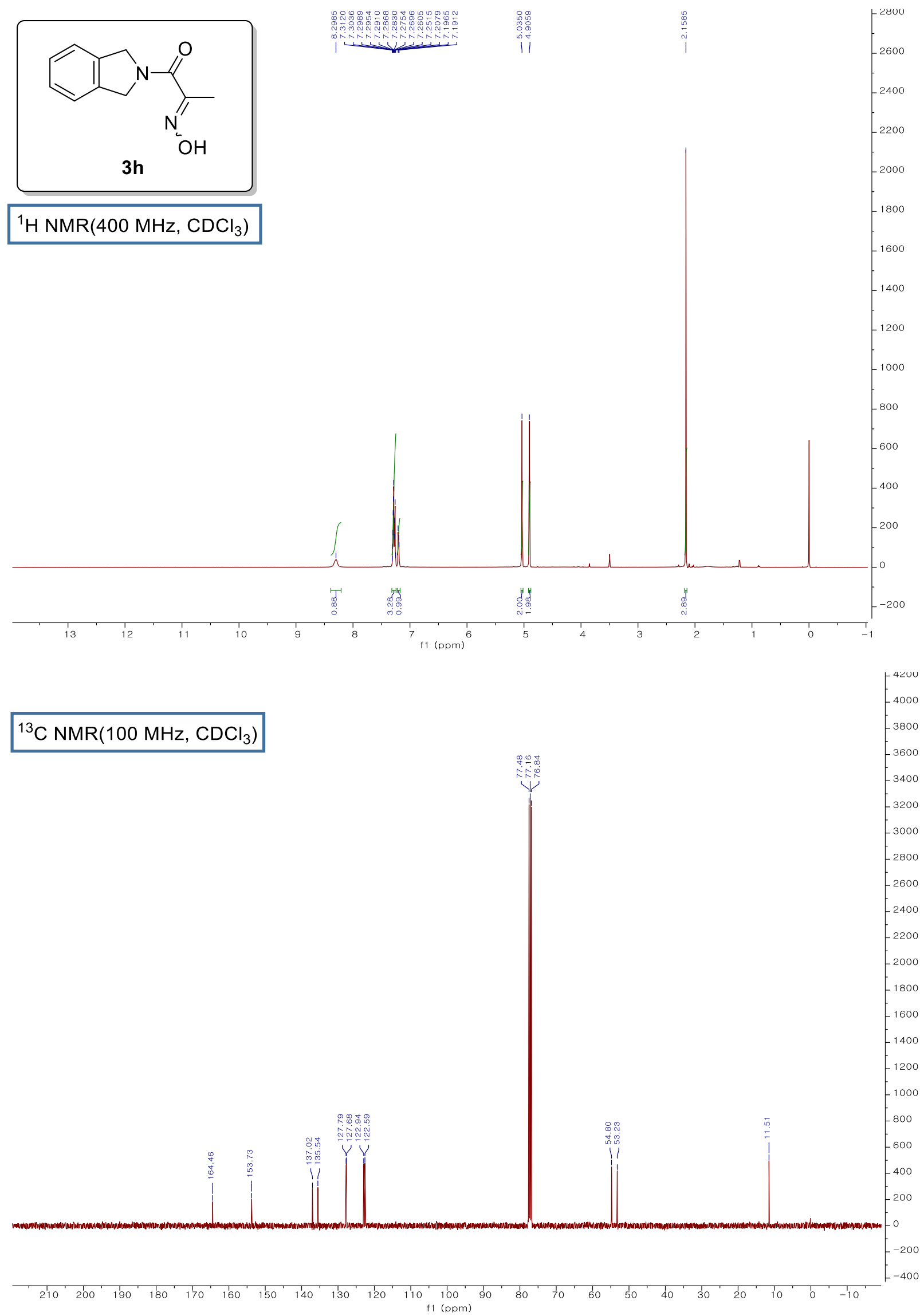

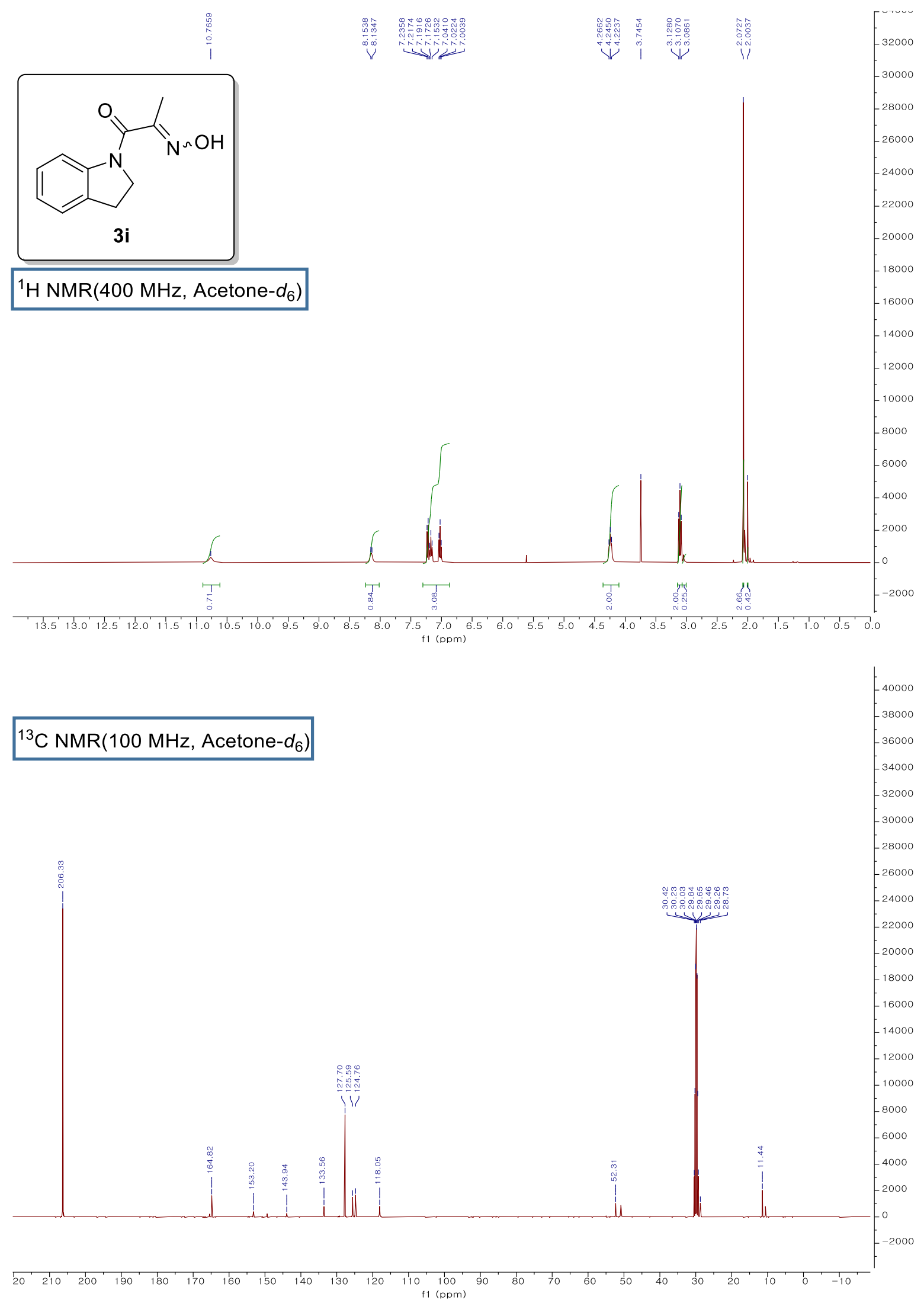

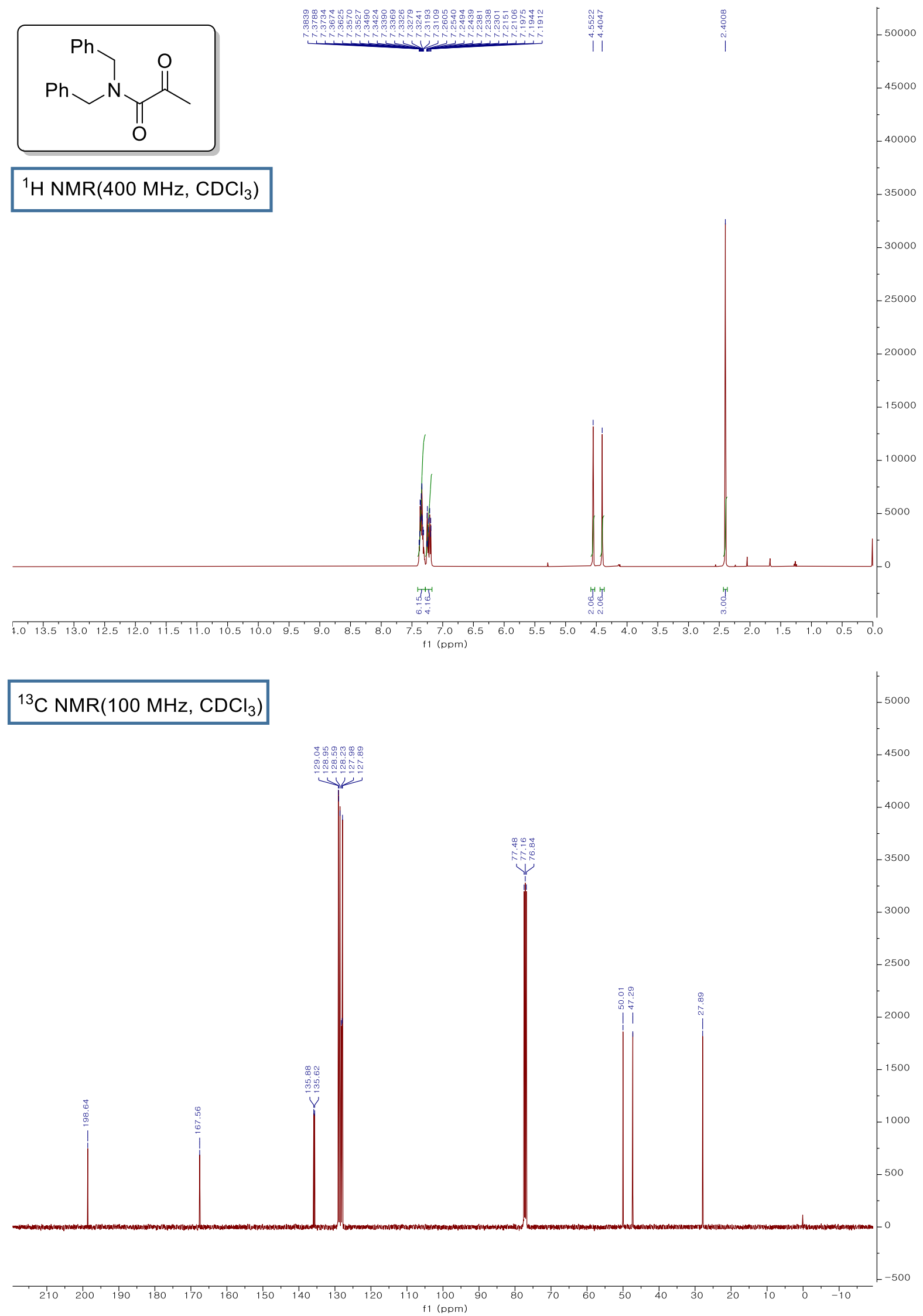

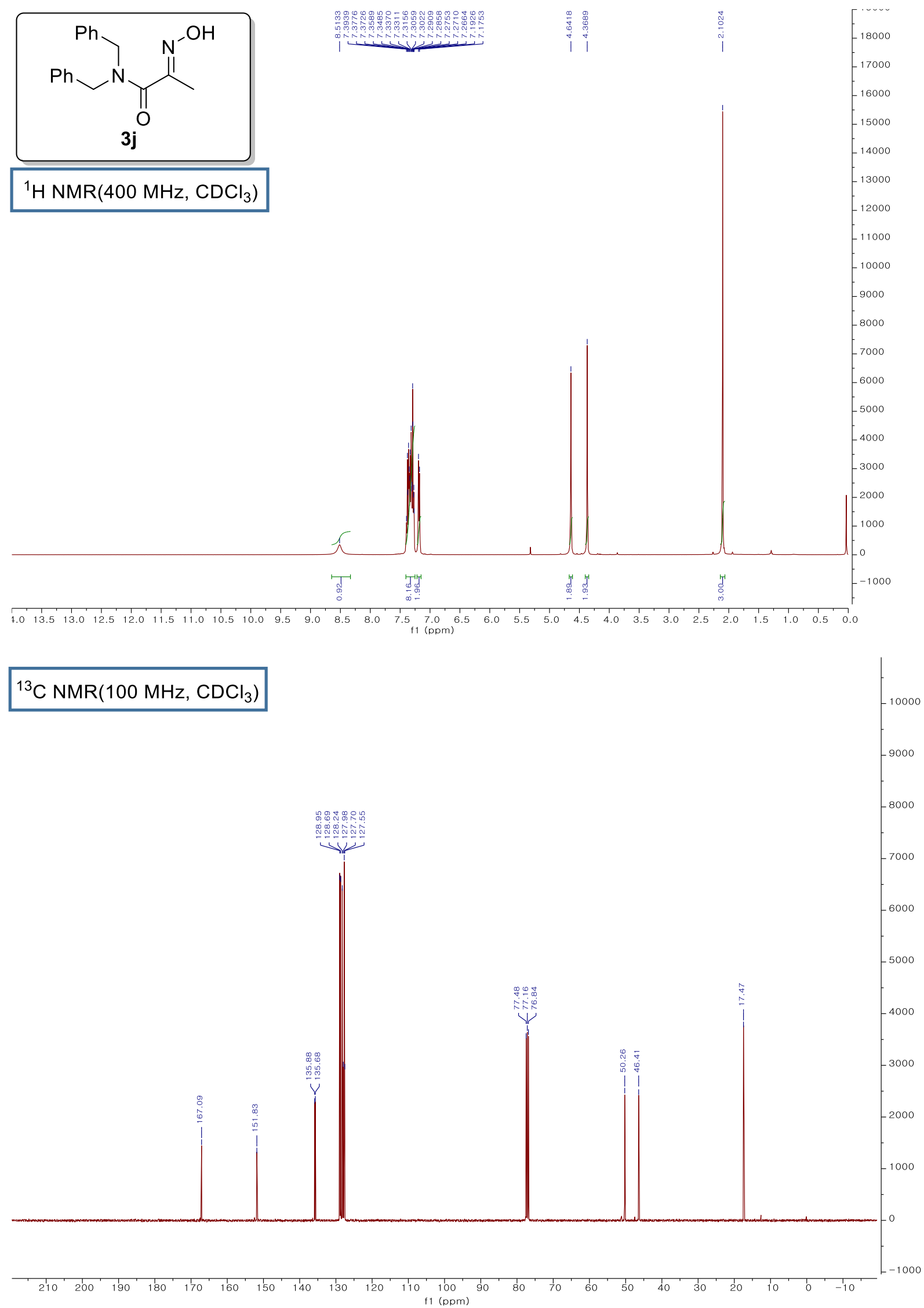


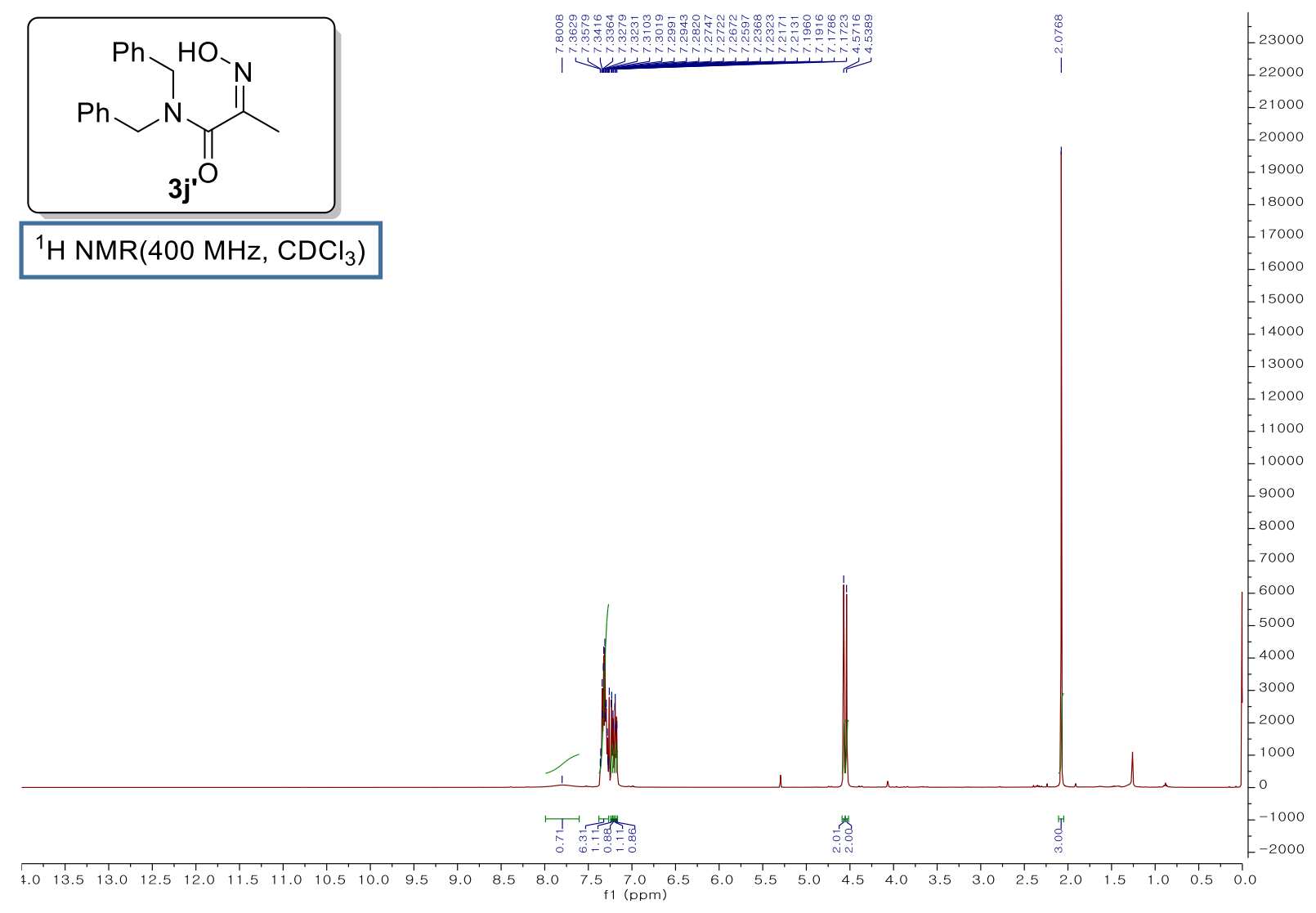

${ }^{13} \mathrm{C} \mathrm{NMR}\left(100 \mathrm{MHz}, \mathrm{CDCl}_{3}\right)$

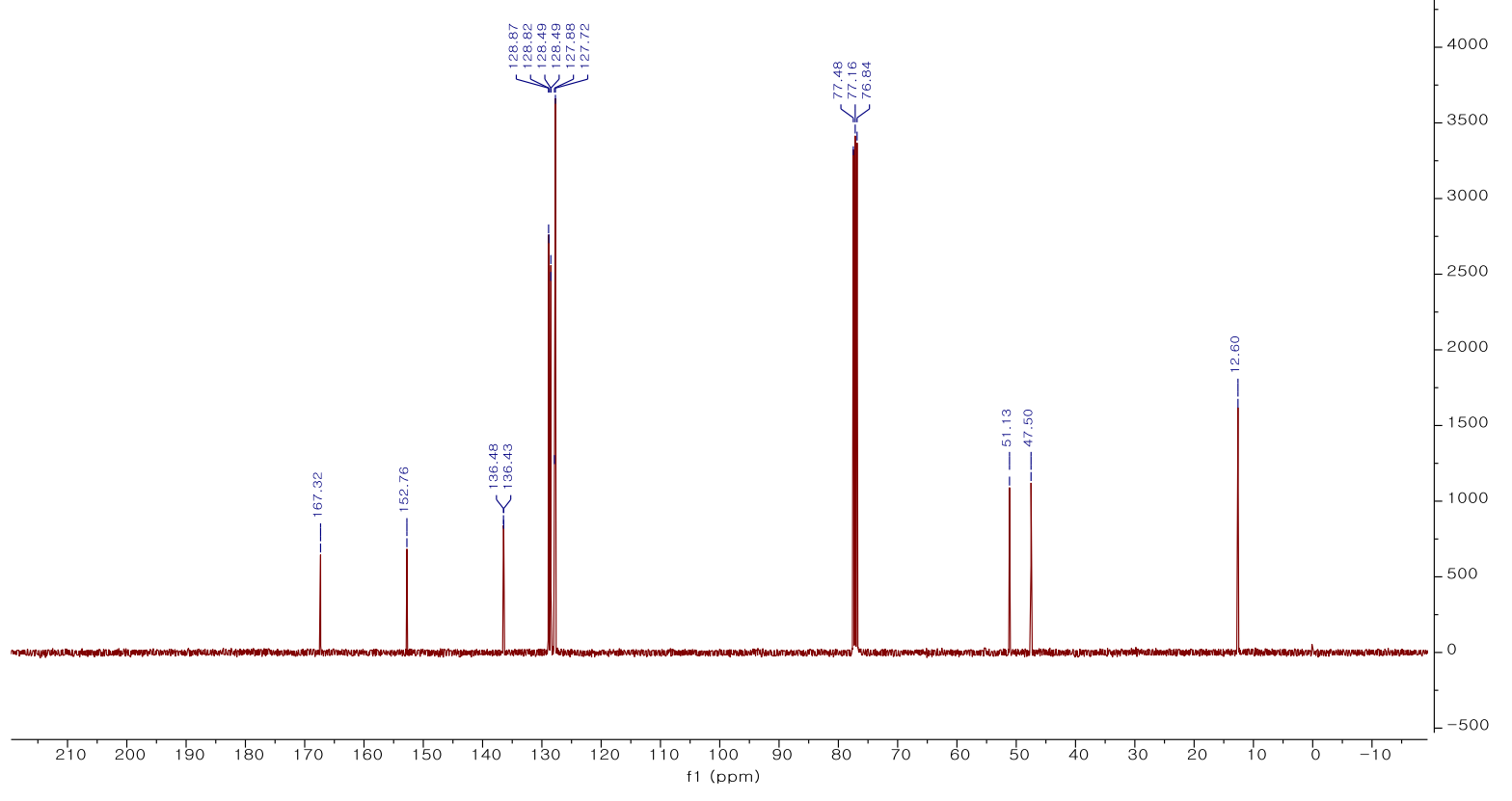




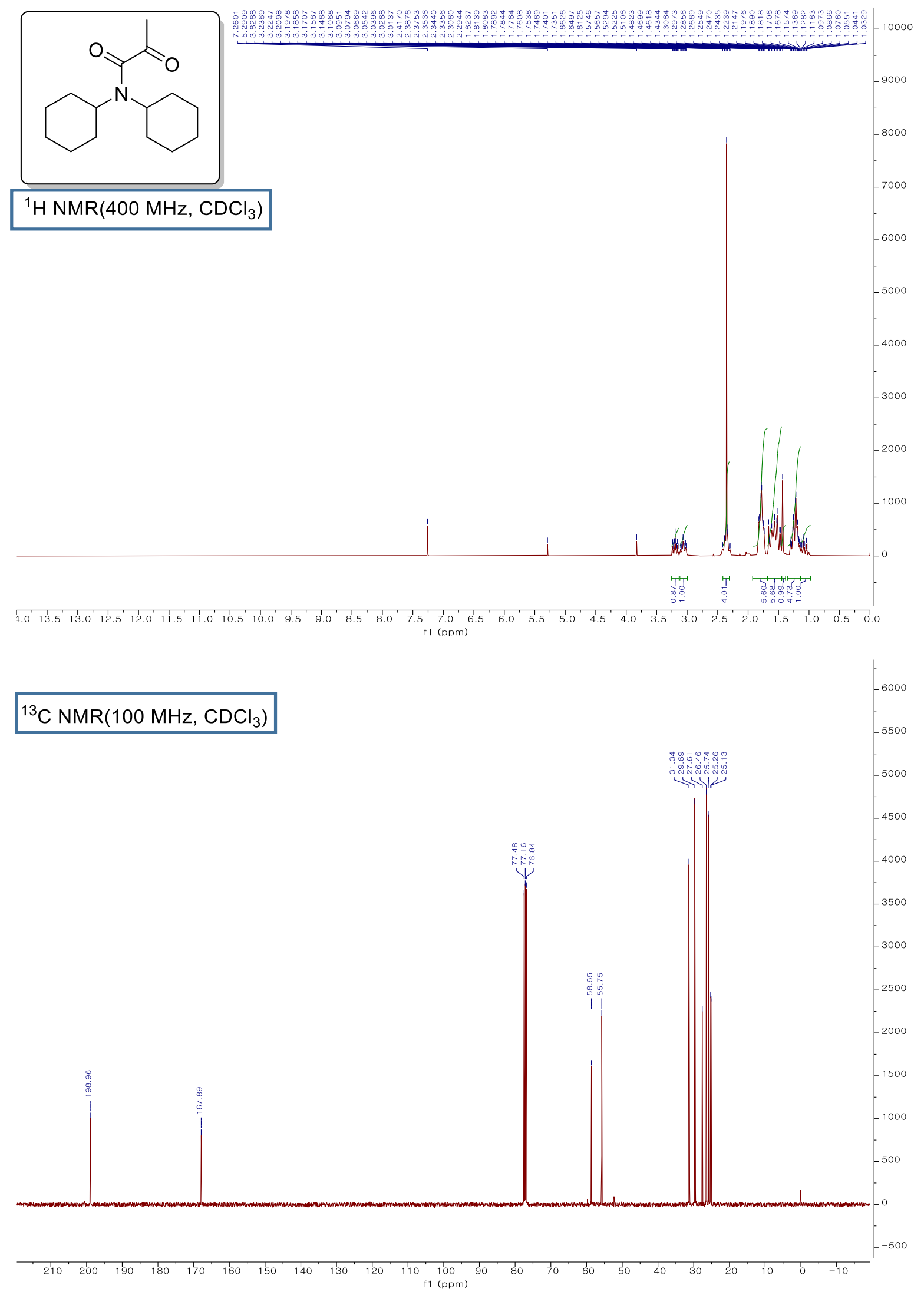




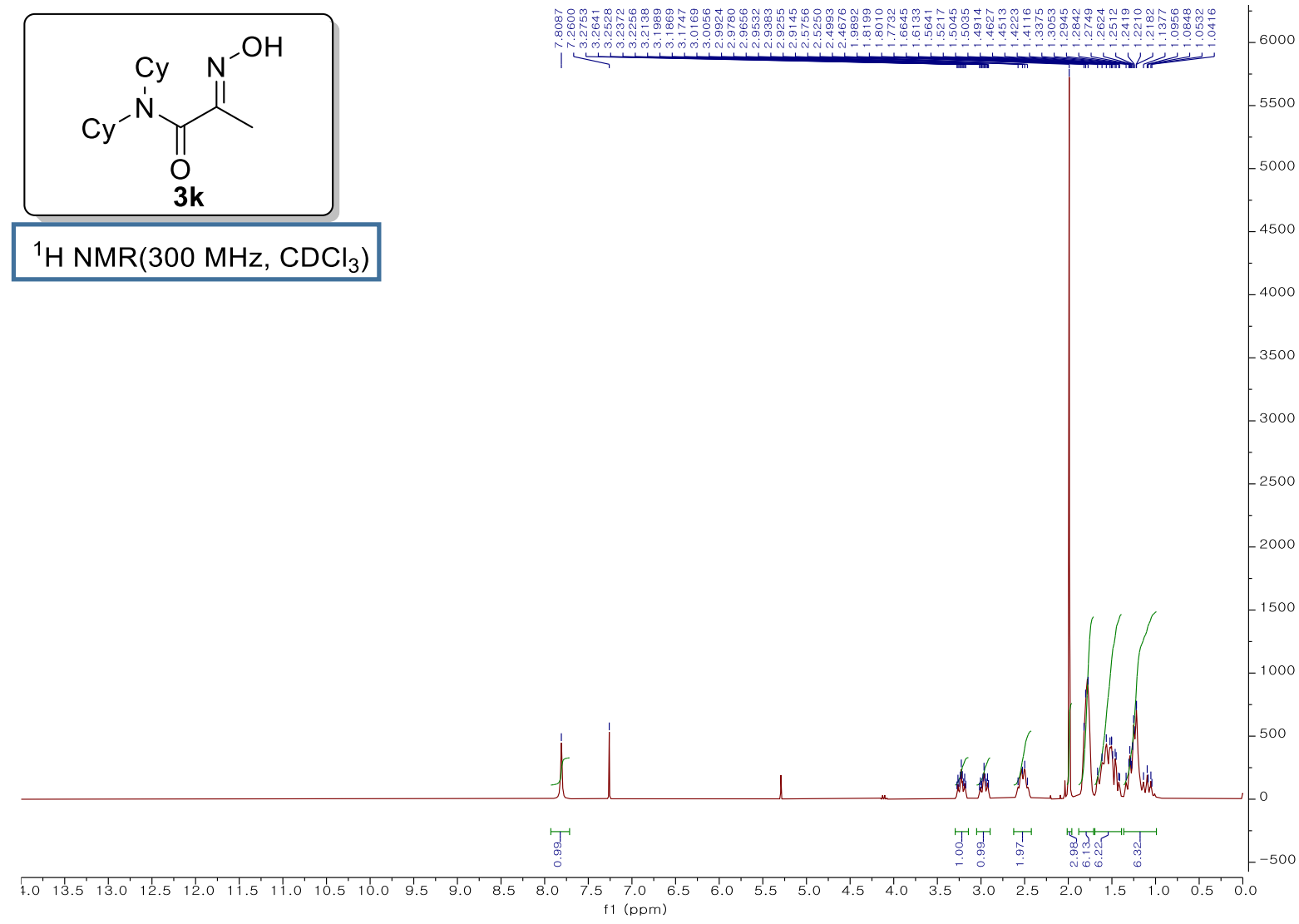

${ }^{13} \mathrm{C} \mathrm{NMR}\left(100 \mathrm{MHz}, \mathrm{CDCl}_{3}\right)$

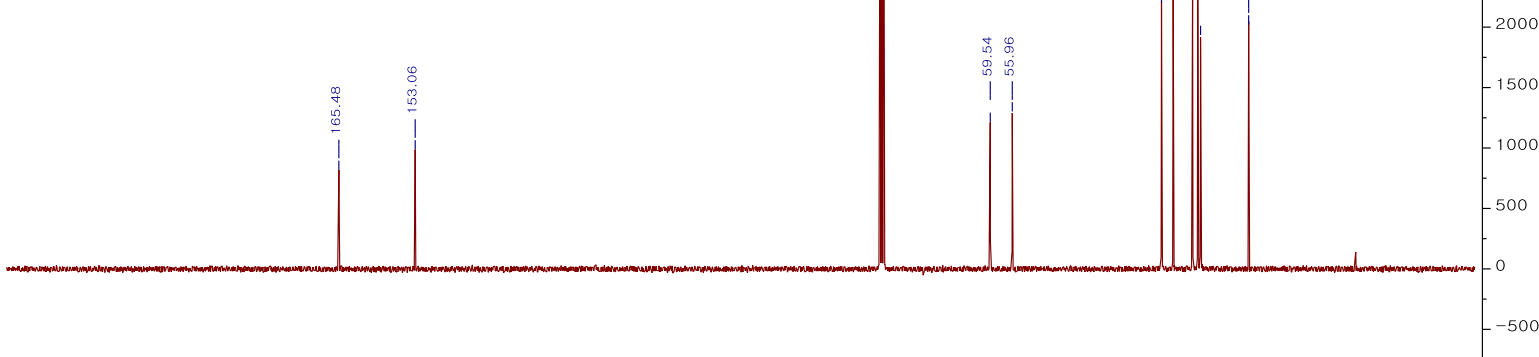

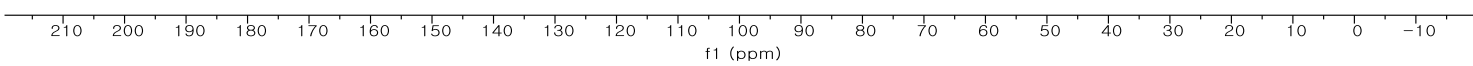




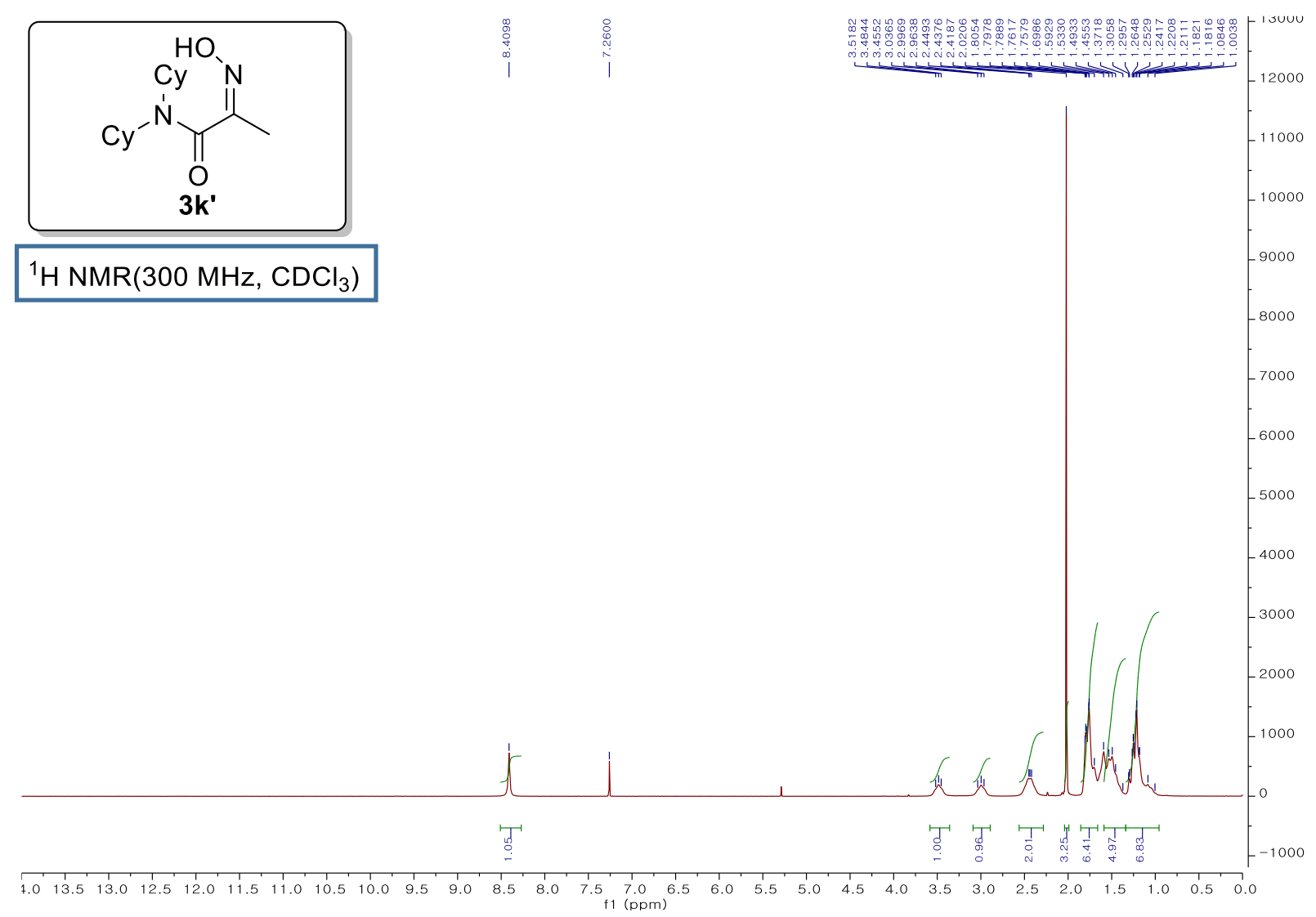

${ }^{13} \mathrm{C} \mathrm{NMR}\left(100 \mathrm{MHz}, \mathrm{CDCl}_{3}\right)$

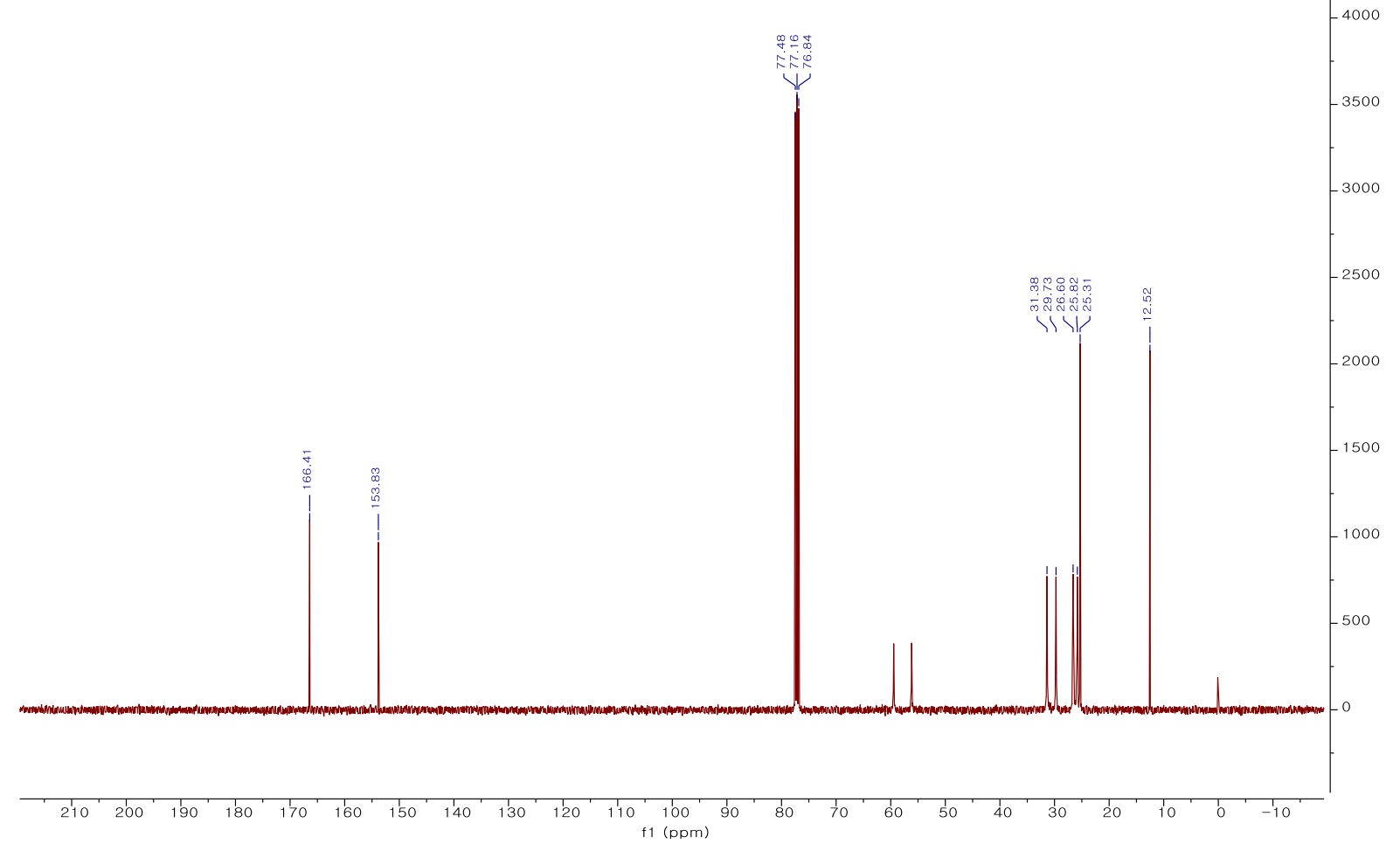



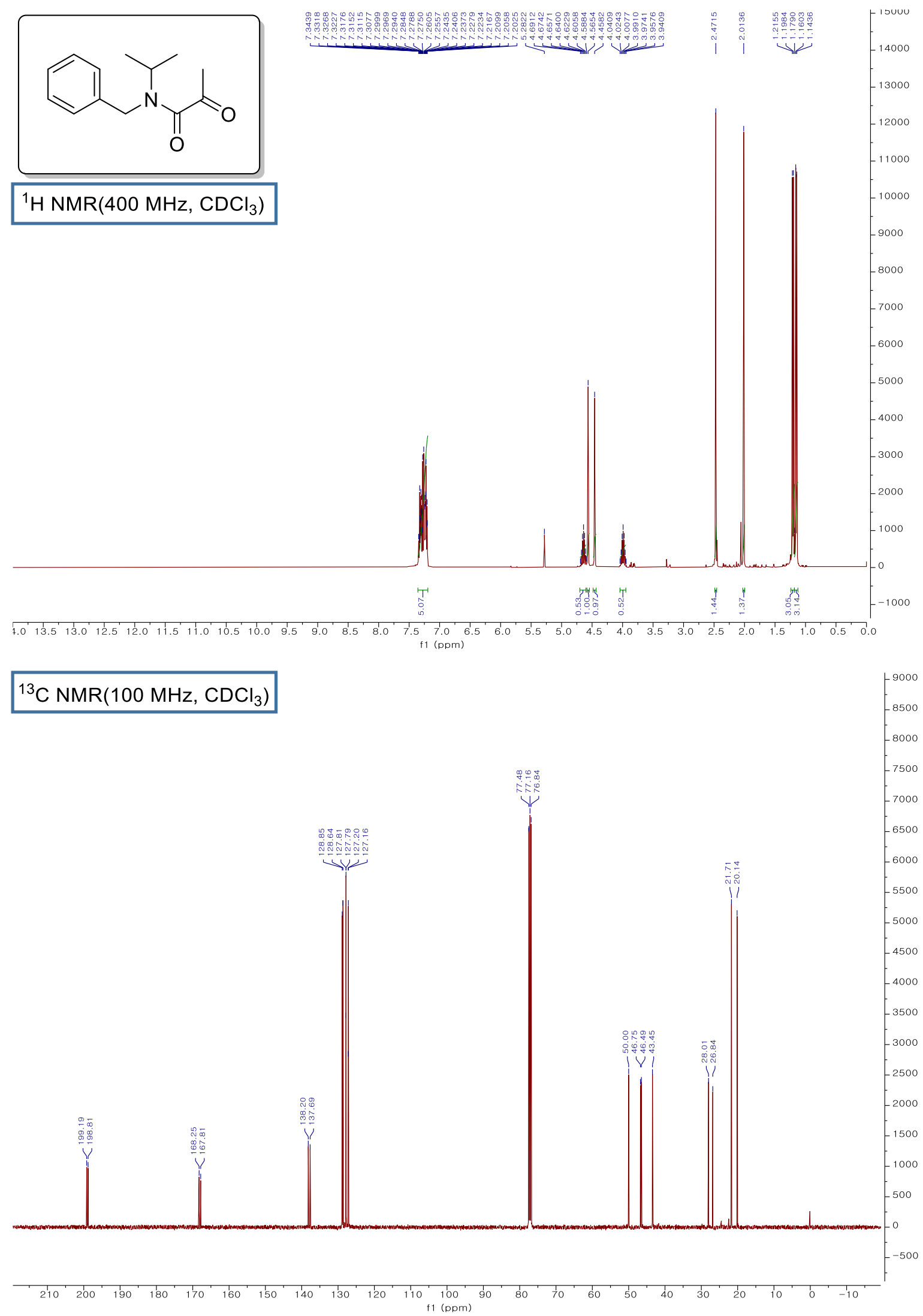

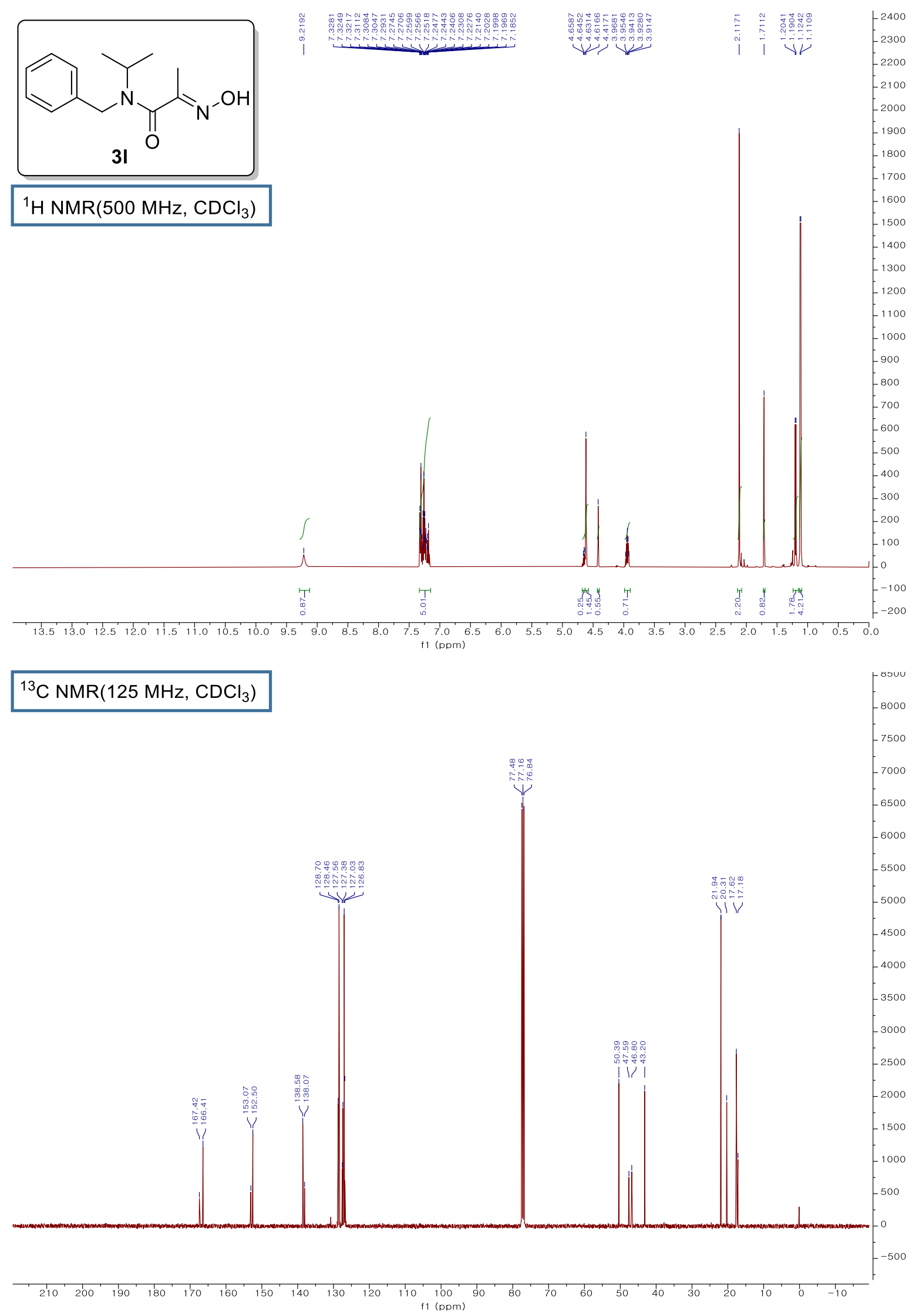

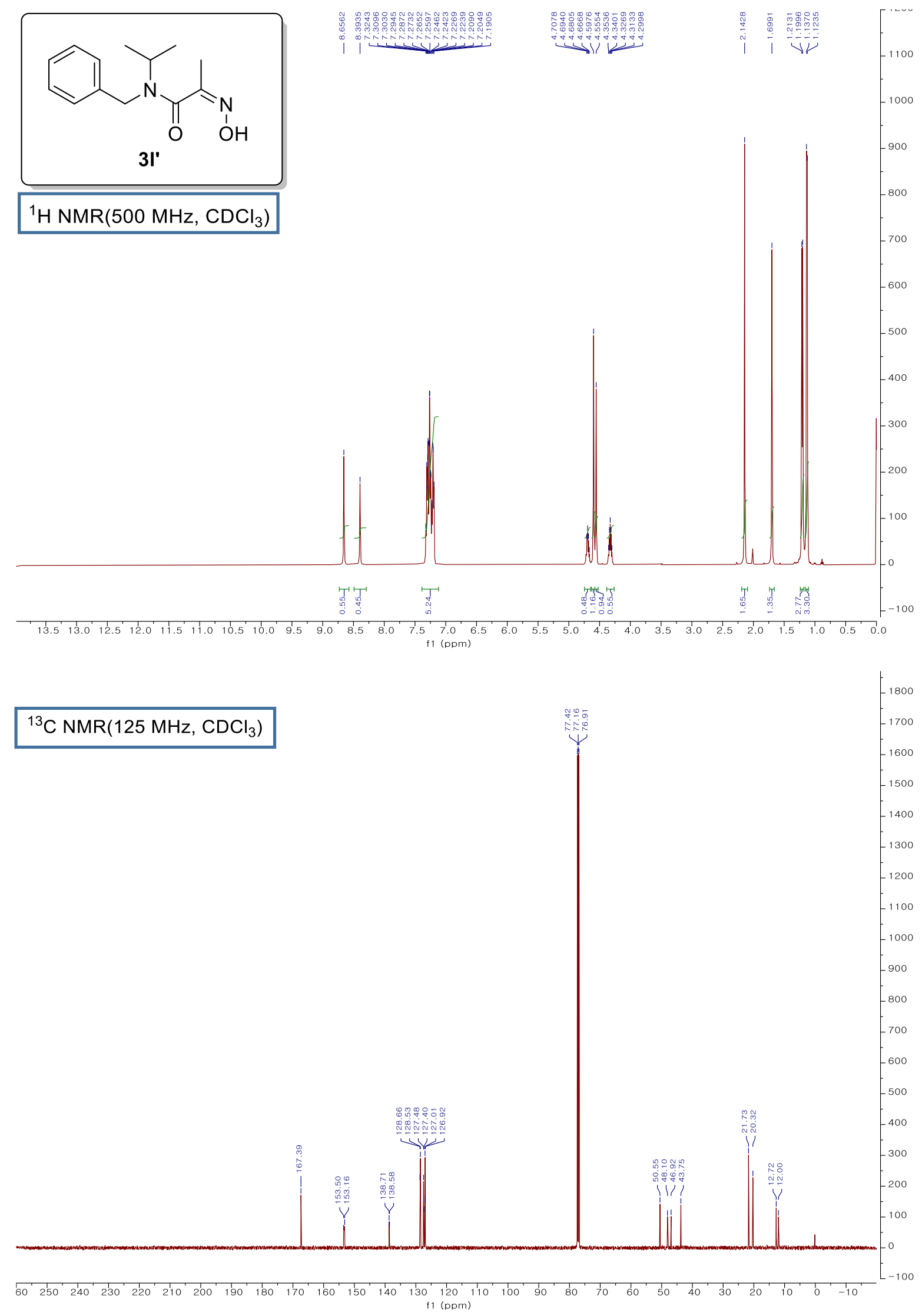

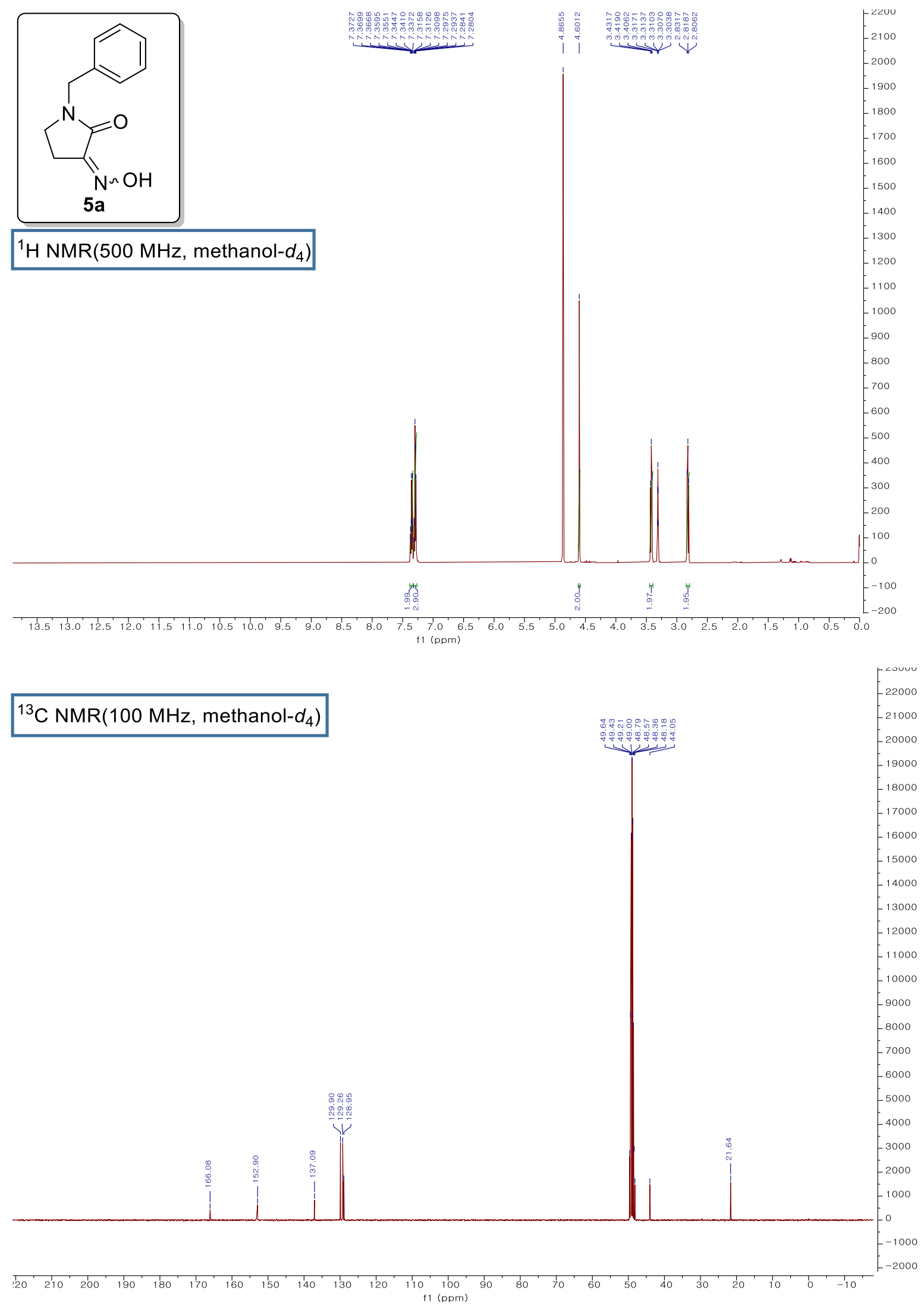

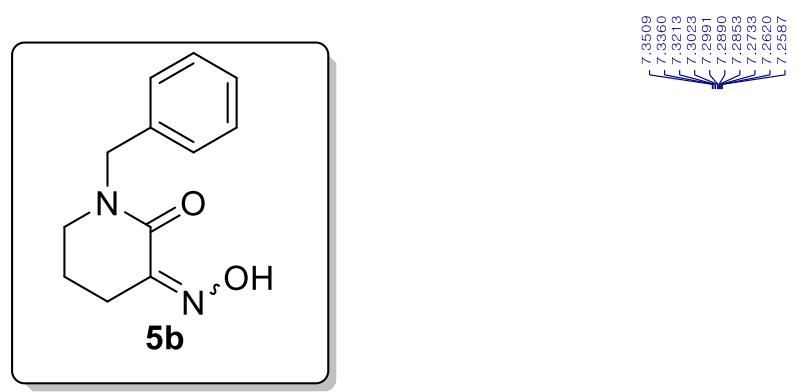

${ }^{1} \mathrm{H}$ NMR(500 MHz, methanol- $\left.d_{4}\right)$
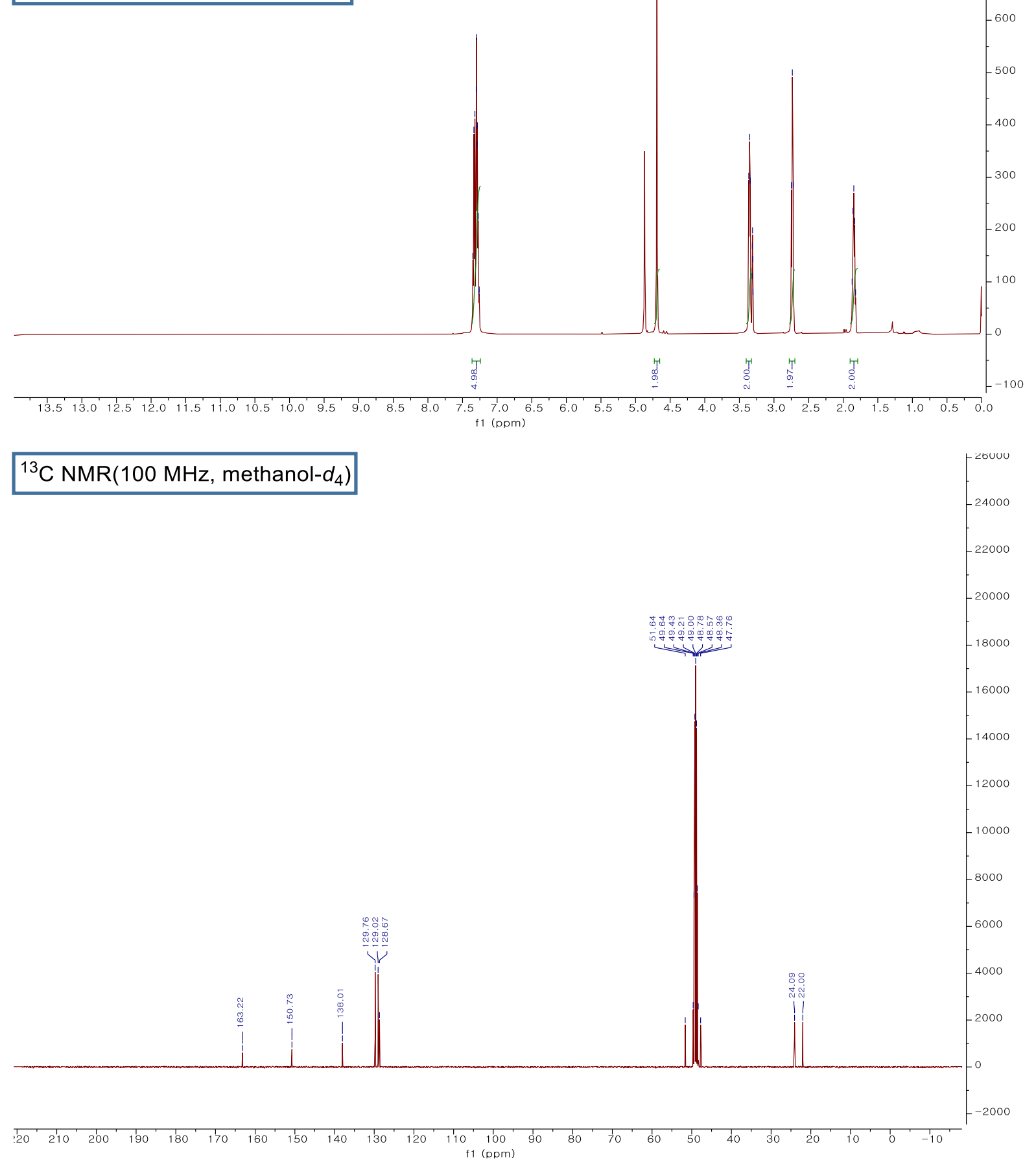

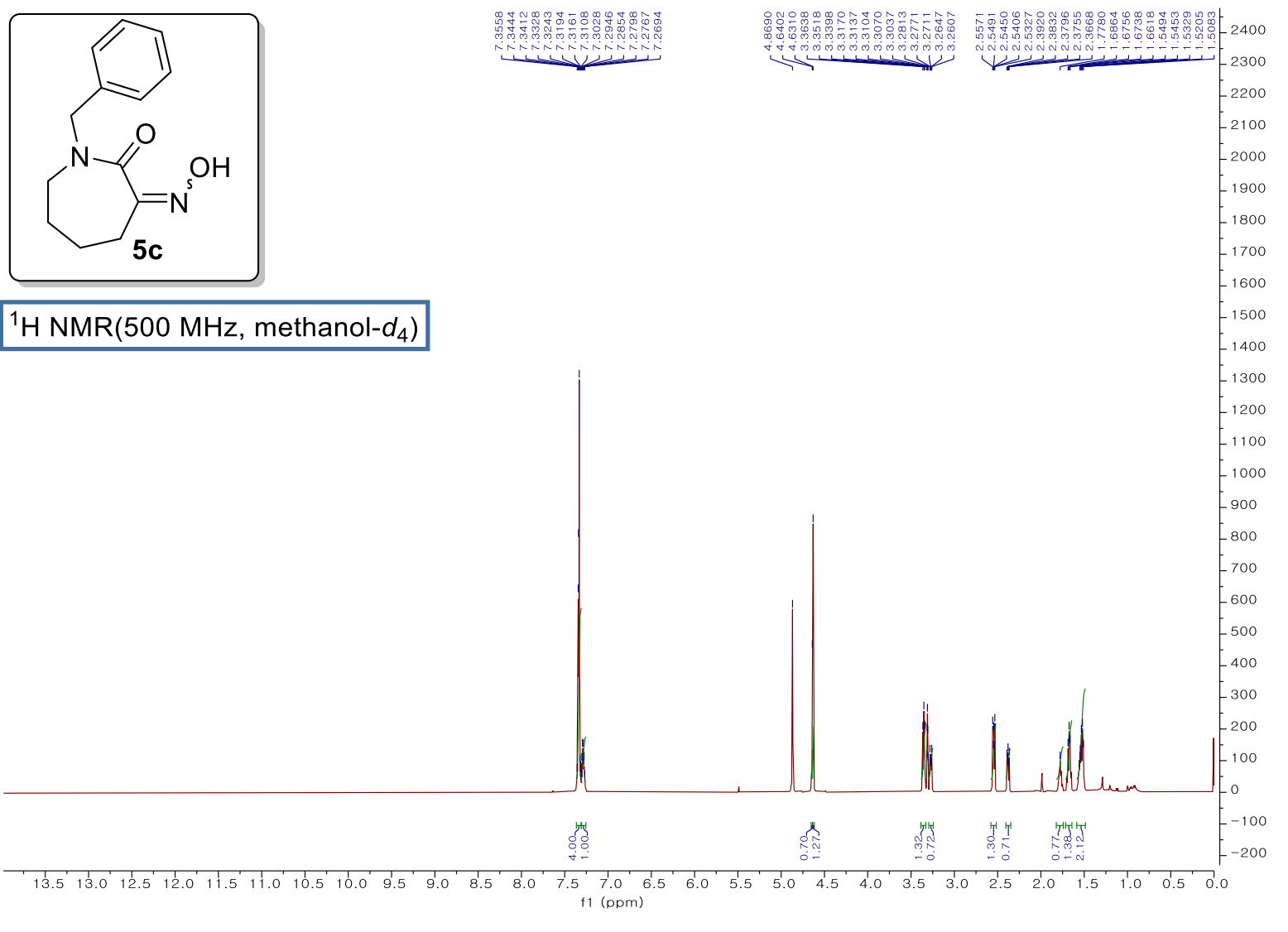

${ }^{13} \mathrm{C}$ NMR(100 MHz, methanol- $\left.d_{4}\right)$

${ }^{1} \mathrm{H}$ NMR $\left(500 \mathrm{MHz}\right.$, methanol- $\left.d_{4}\right)$
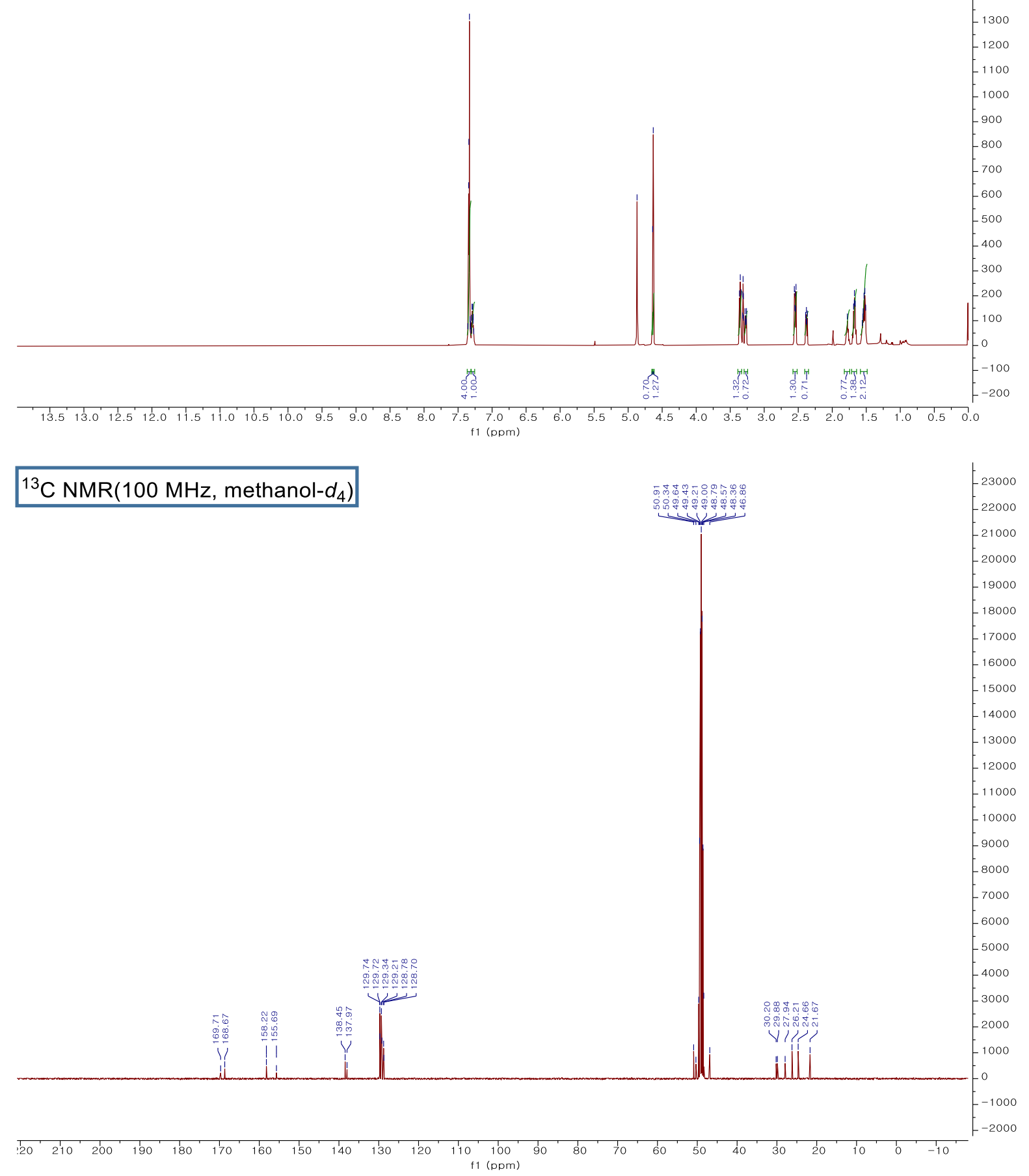


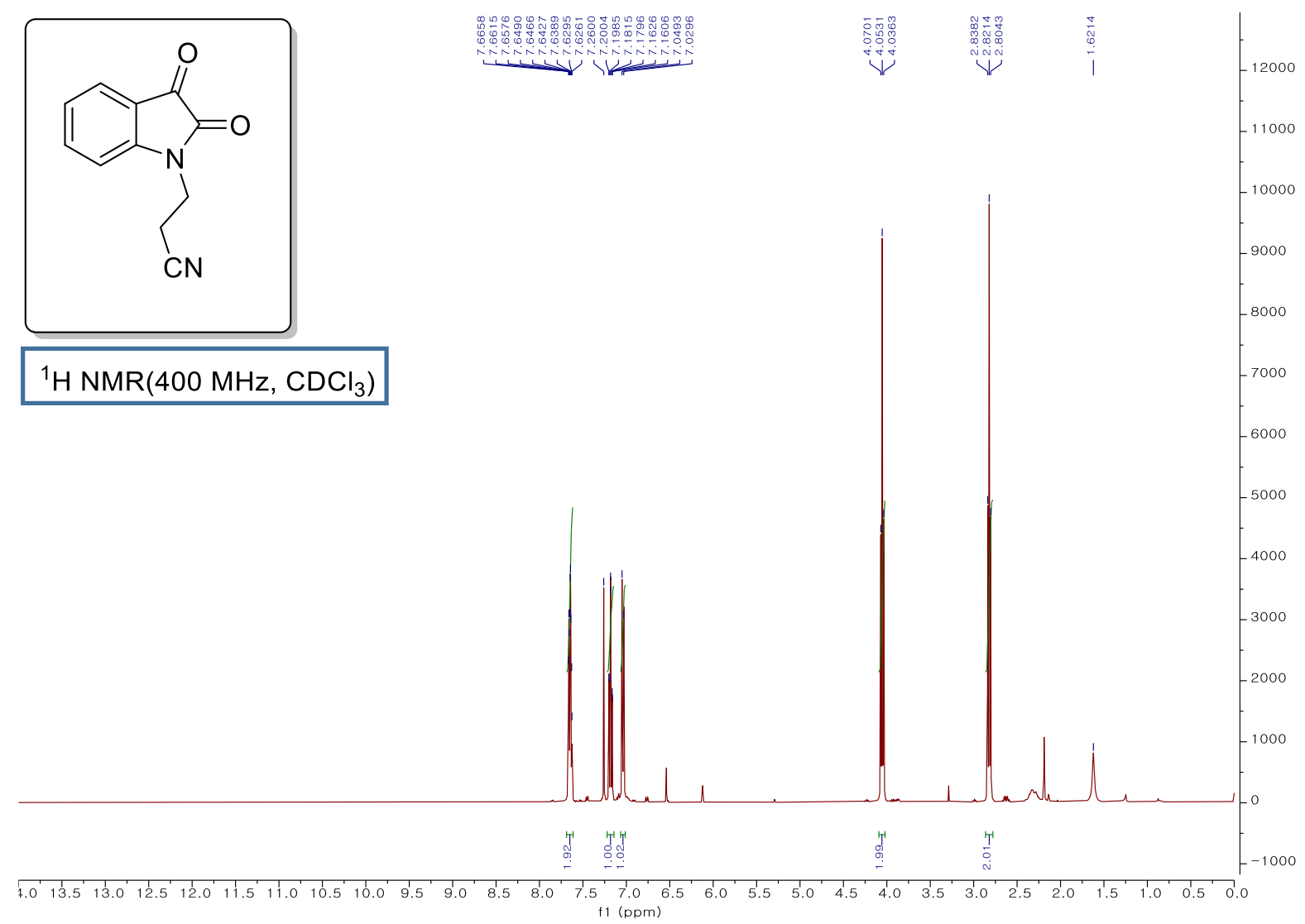

${ }^{13} \mathrm{C} \mathrm{NMR}\left(100 \mathrm{MHz}, \mathrm{CDCl}_{3}\right)$

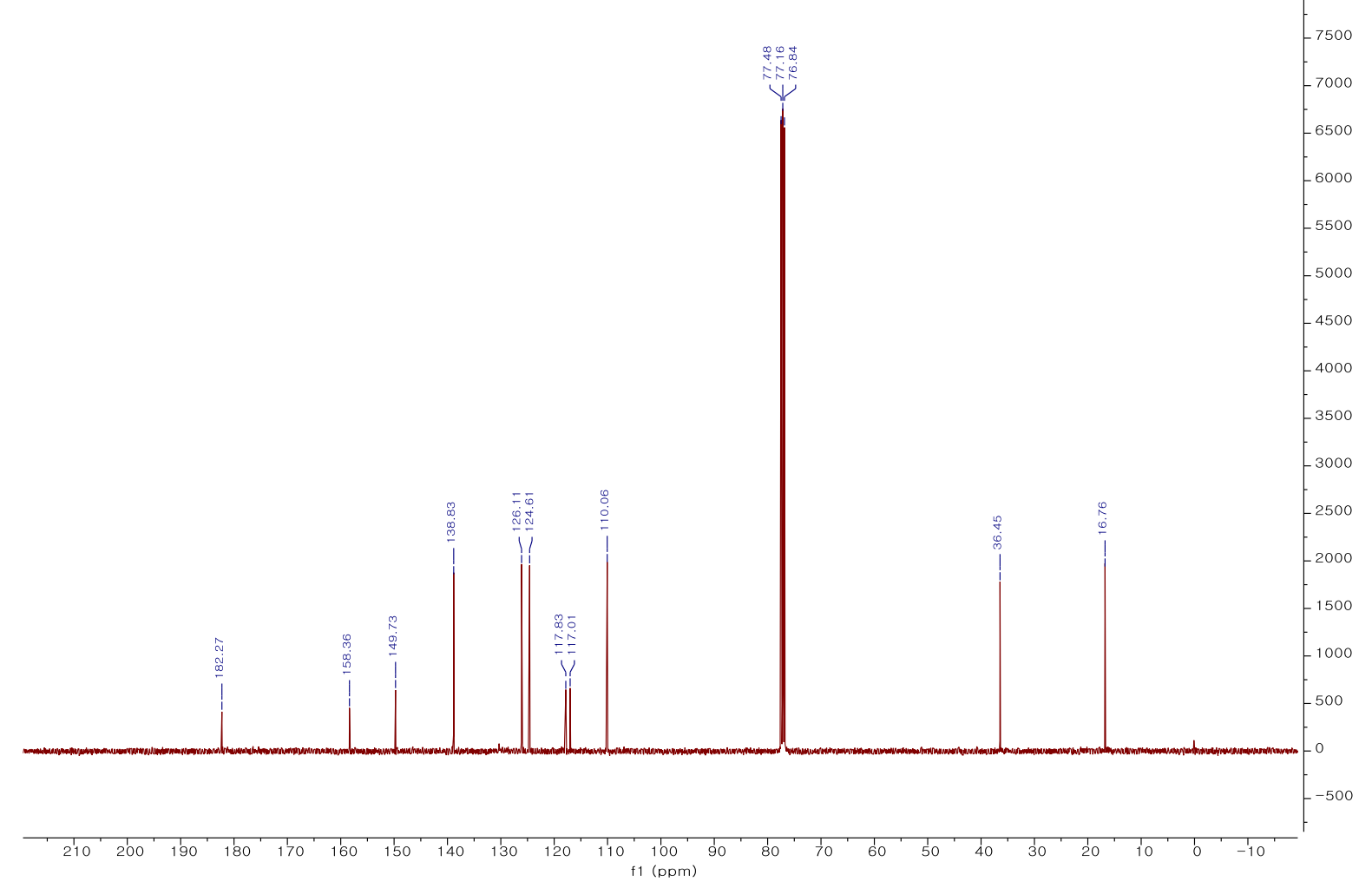



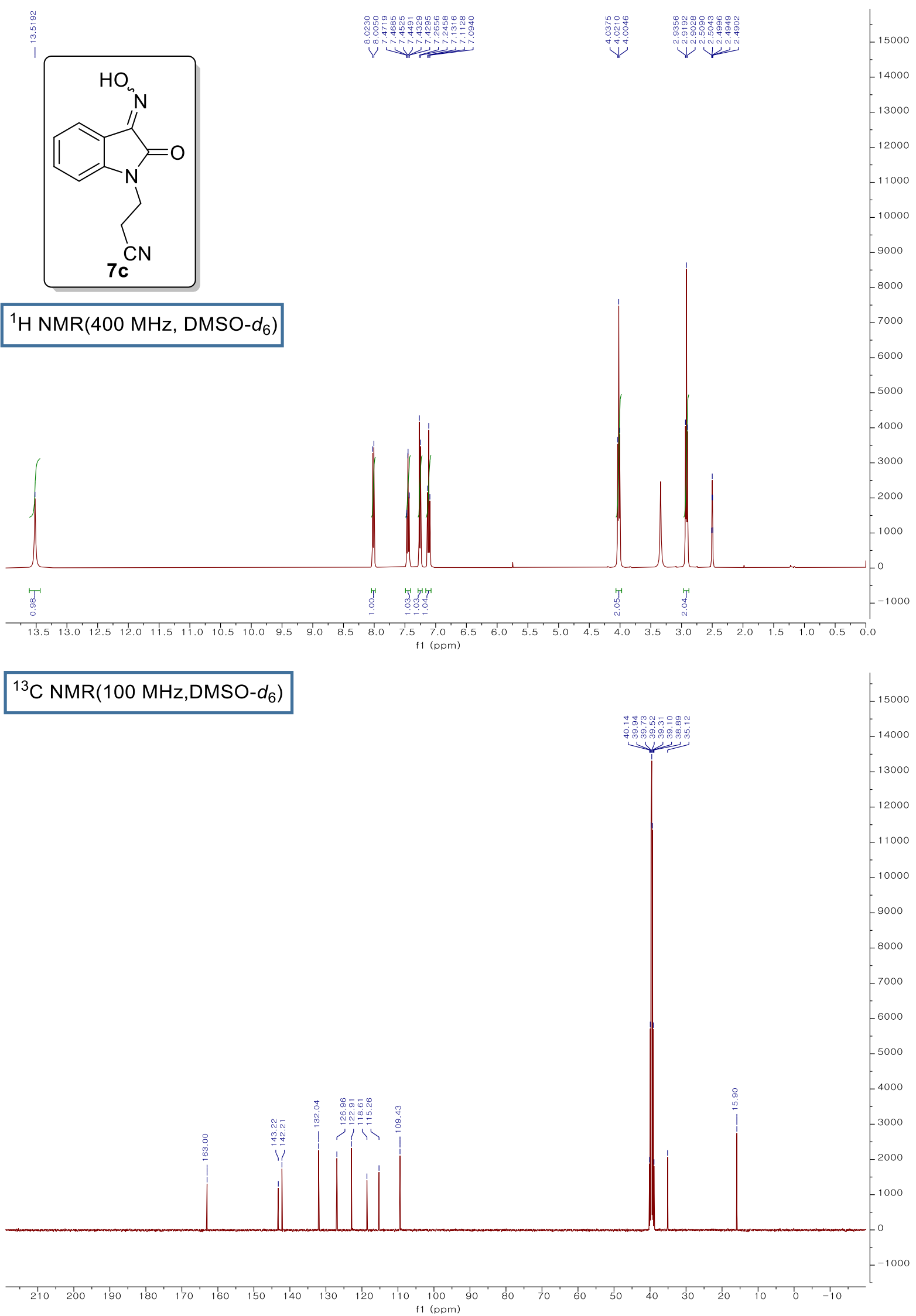

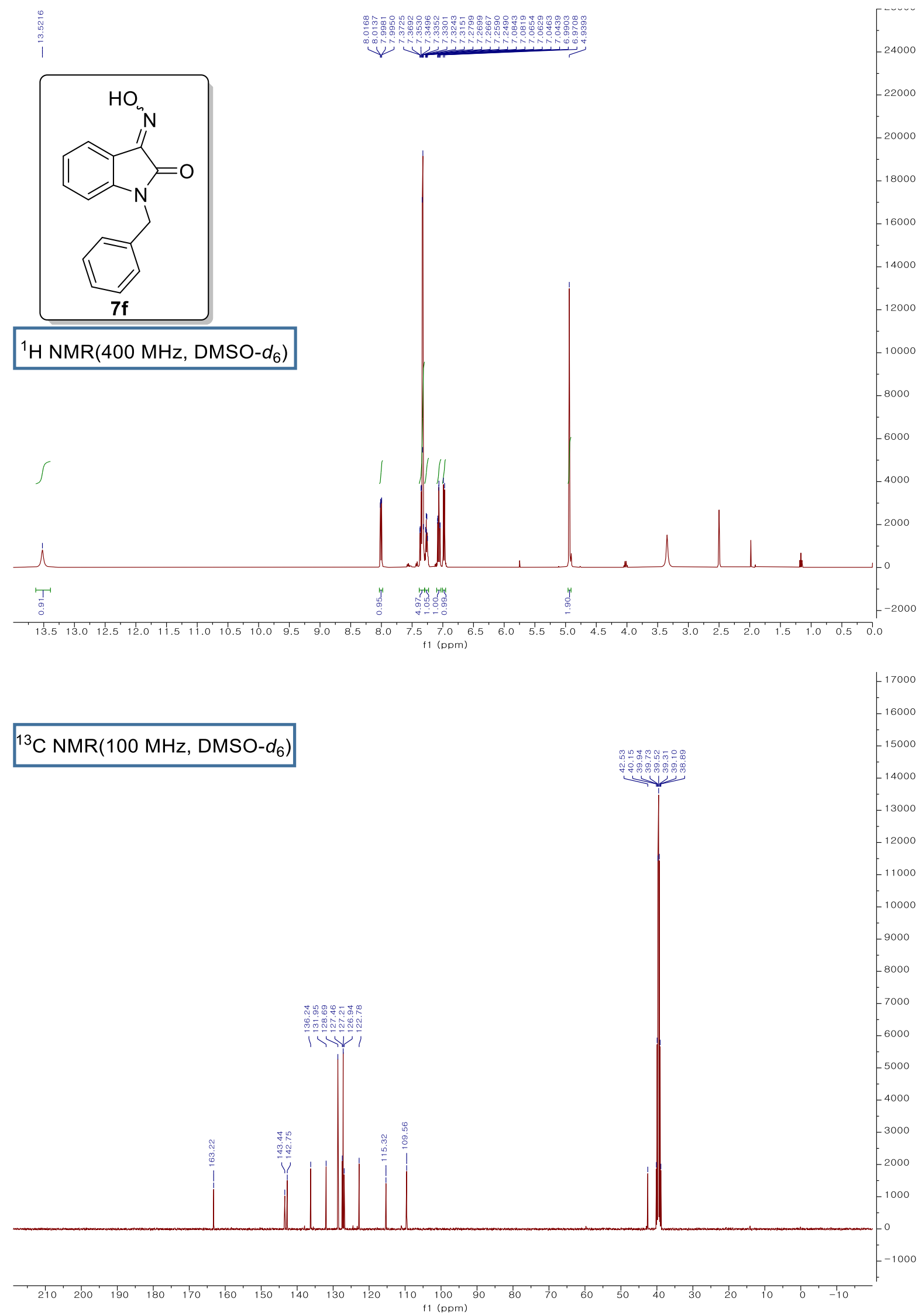


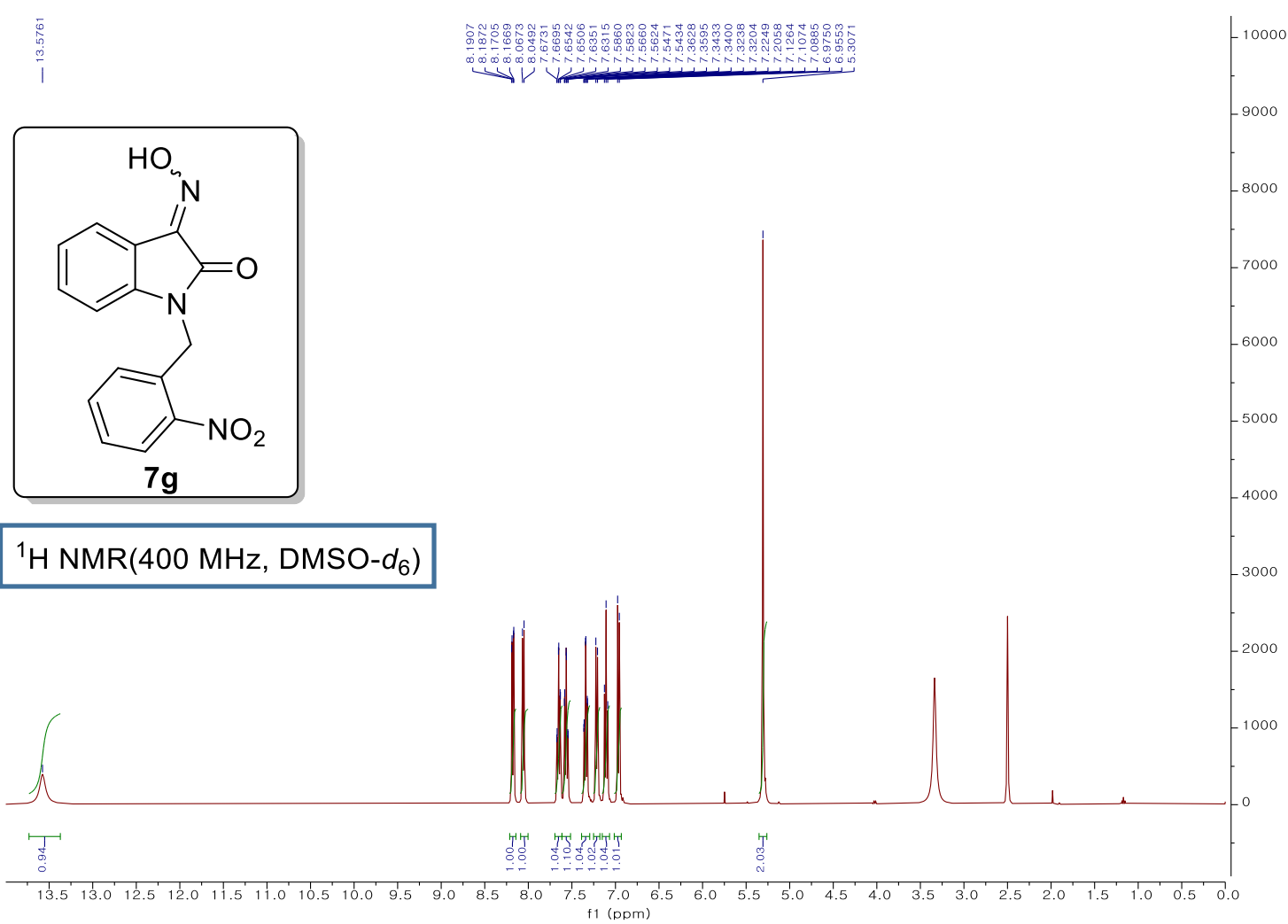

${ }^{13} \mathrm{C} N M R\left(100 \mathrm{MHz}\right.$, DMSO- $\left.d_{6}\right)$

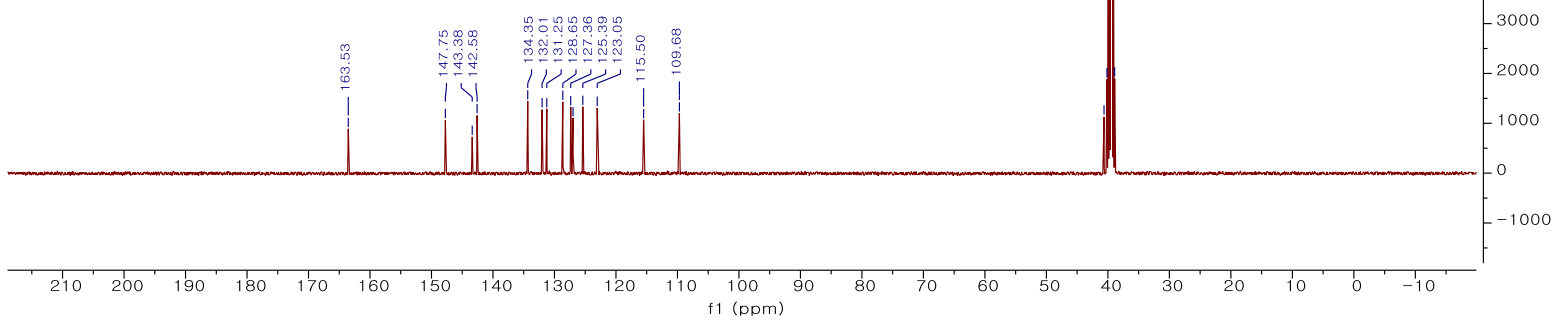




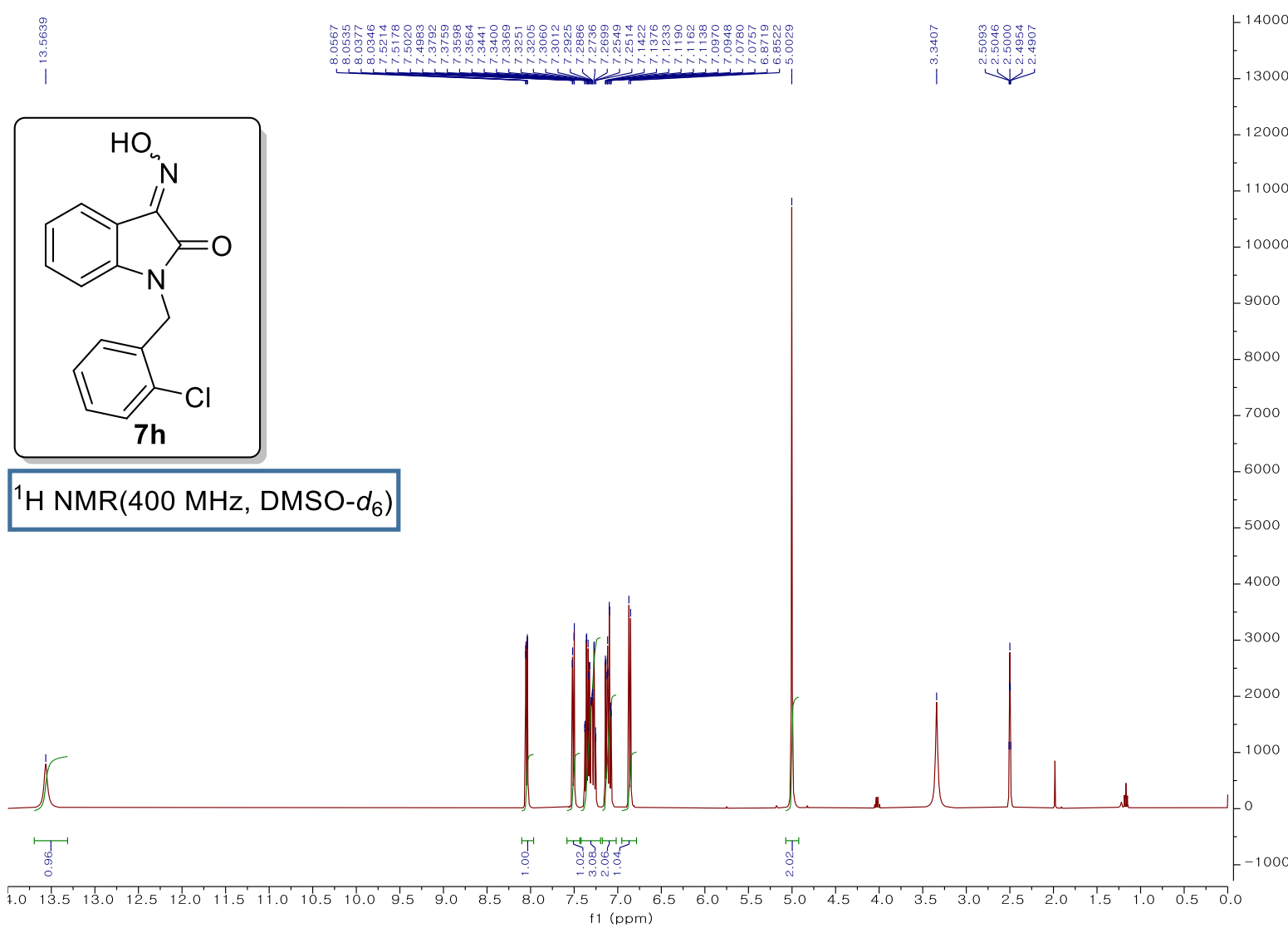

${ }^{13} \mathrm{C}$ NMR(100 MHz, DMSO- $\left.d_{6}\right)$

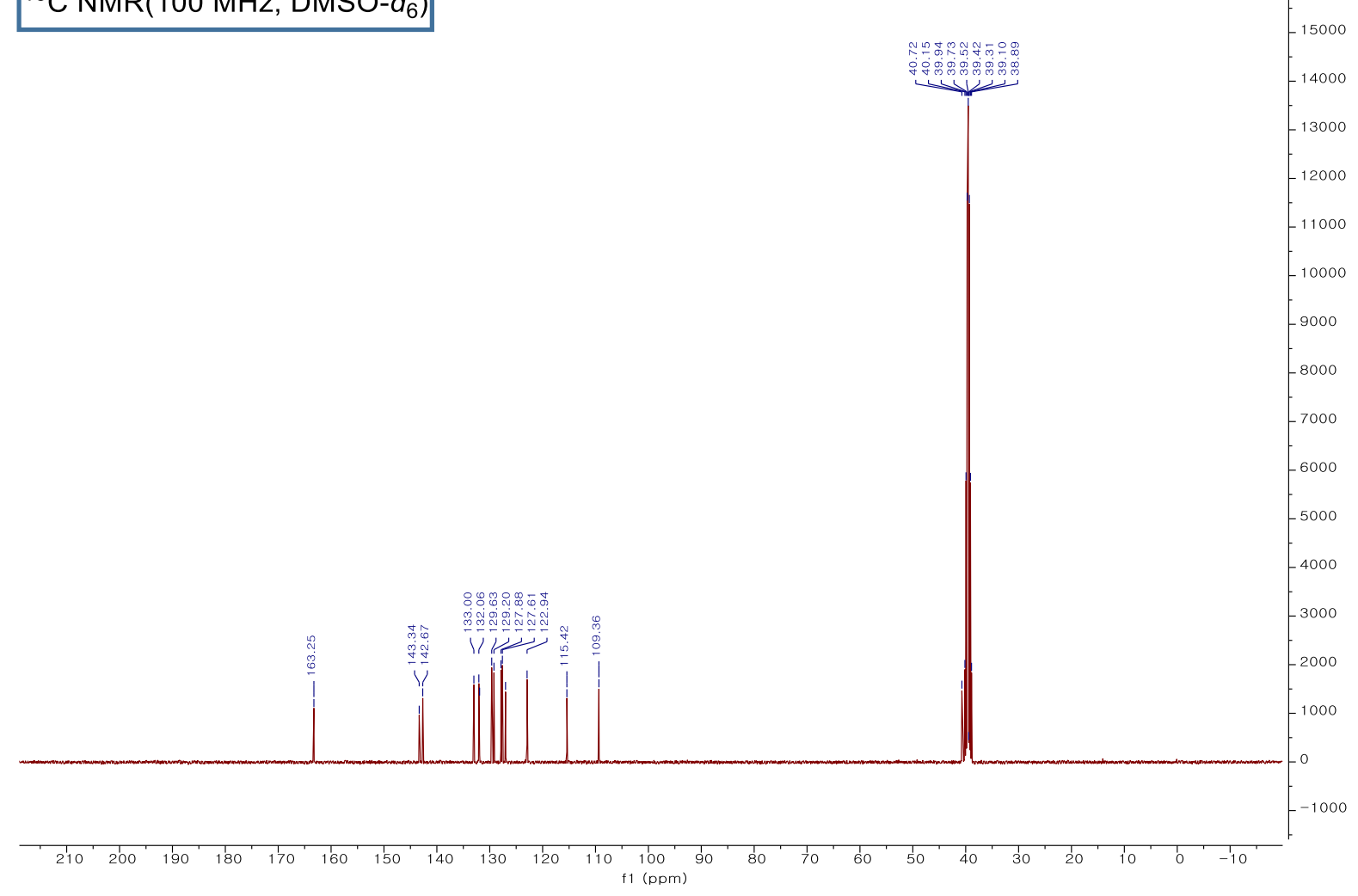




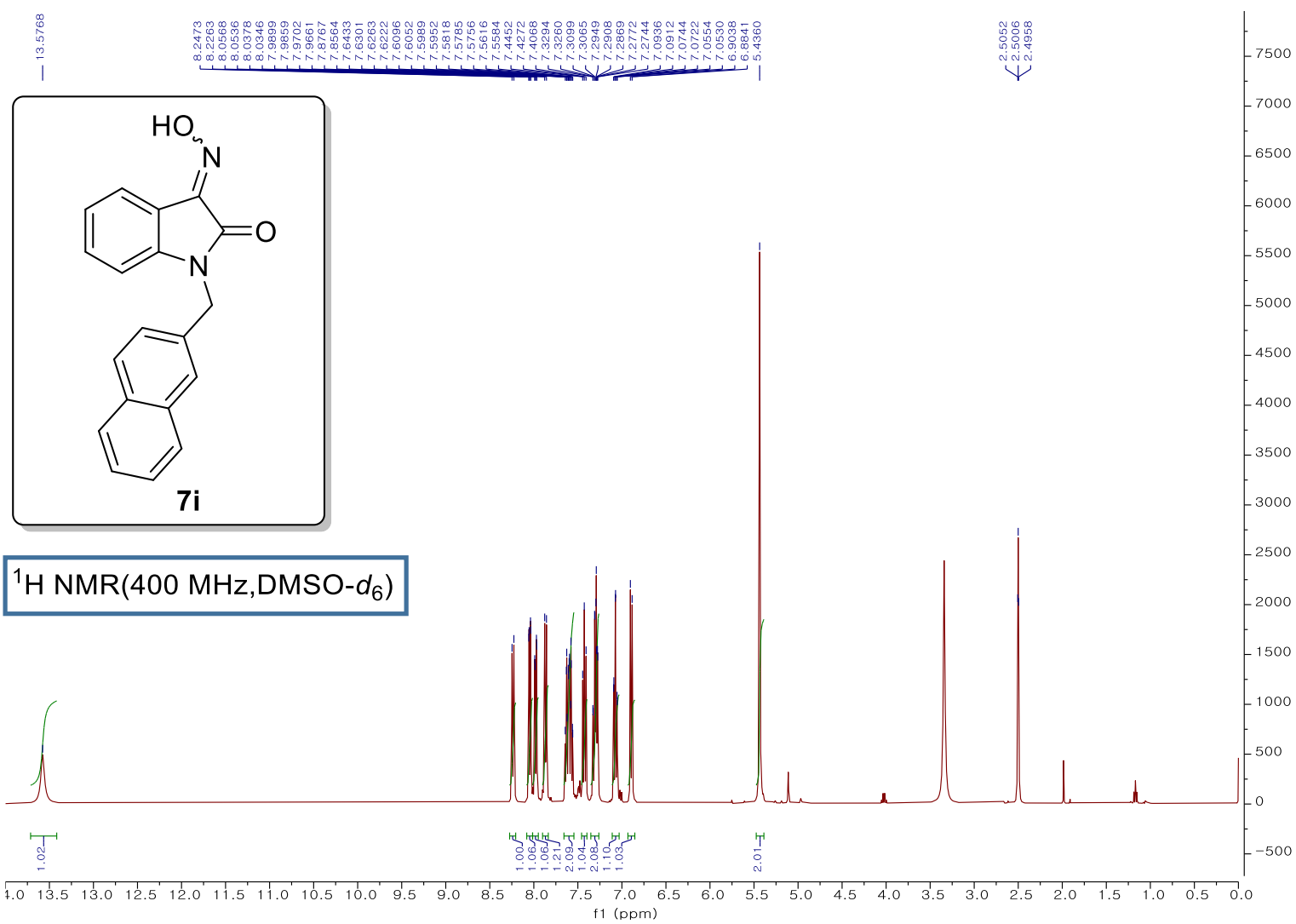

${ }^{13} \mathrm{C}$ NMR(100 MHz, DMSO- $\left.d_{6}\right)$

f1 (ppm)

$-15000$

14000

13000

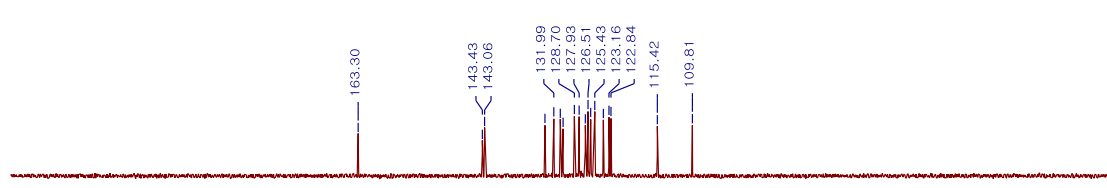



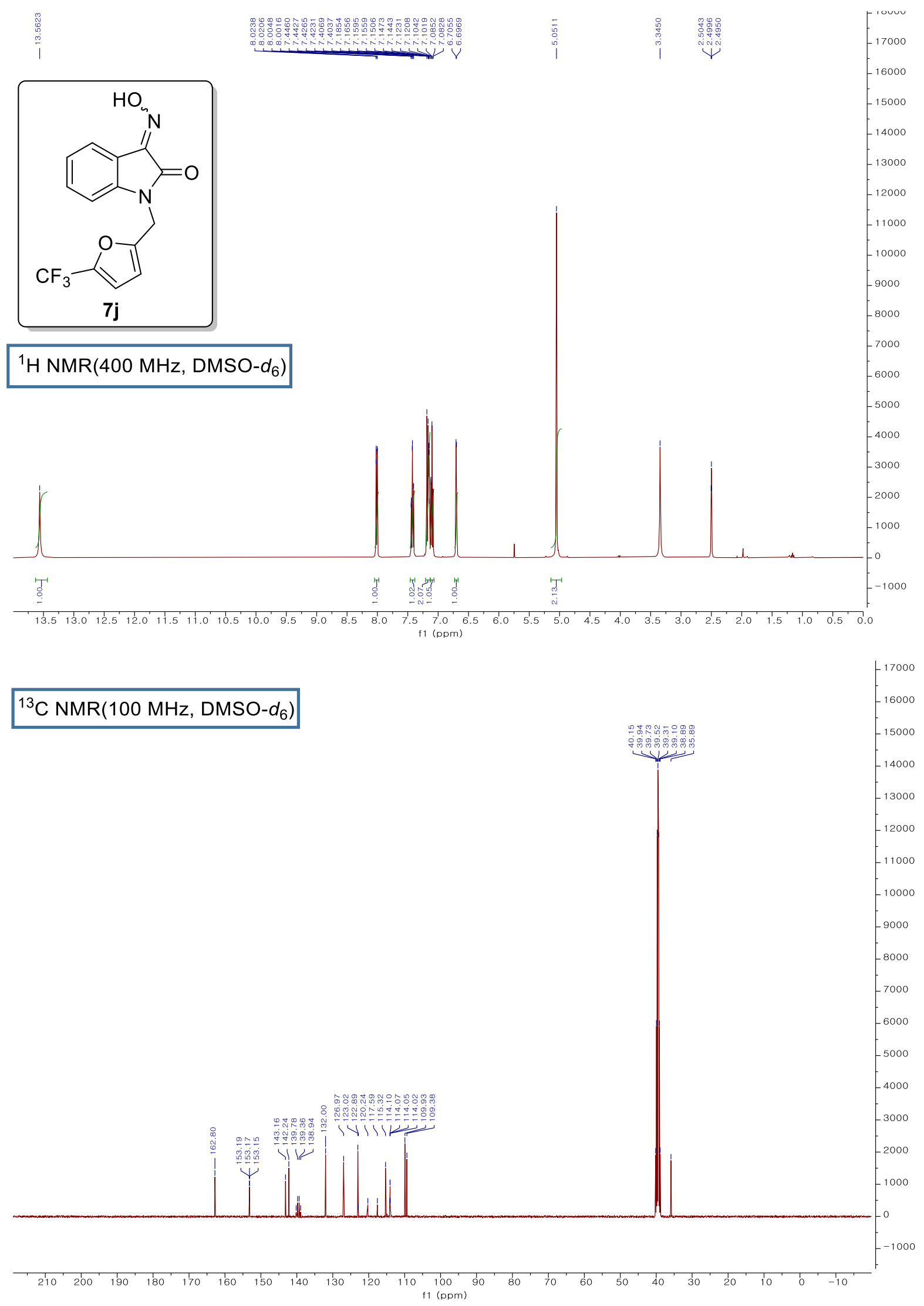

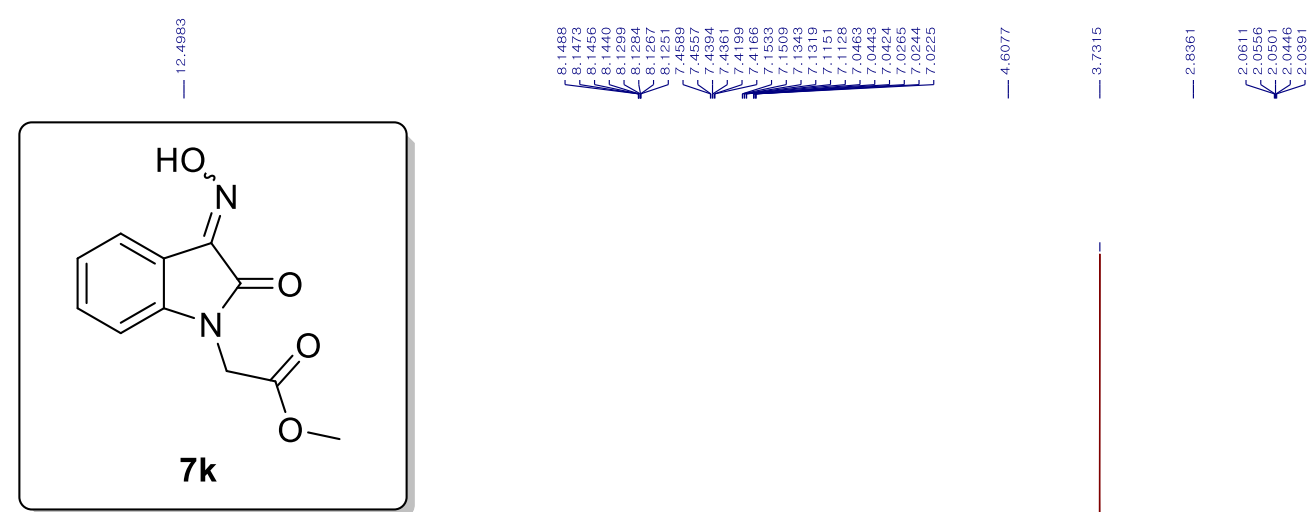

$-26000$

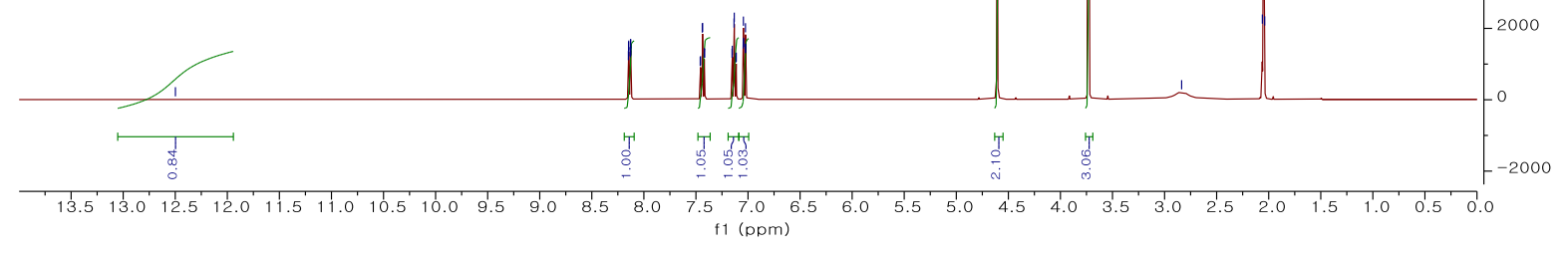

\section{${ }^{13} \mathrm{C} \operatorname{NMR}\left(100 \mathrm{MHz}\right.$, Acetone- $\left.d_{6}\right)$}

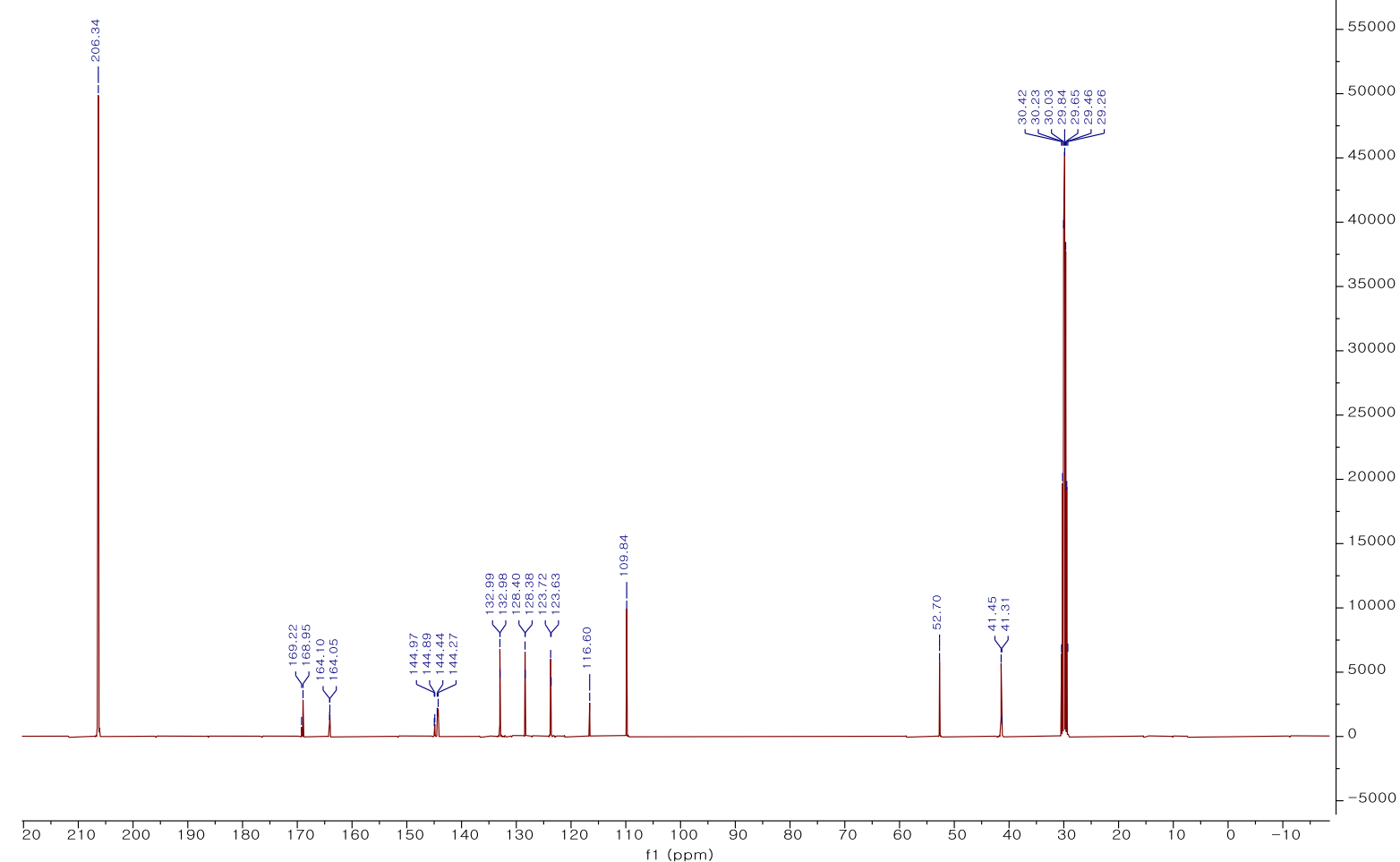



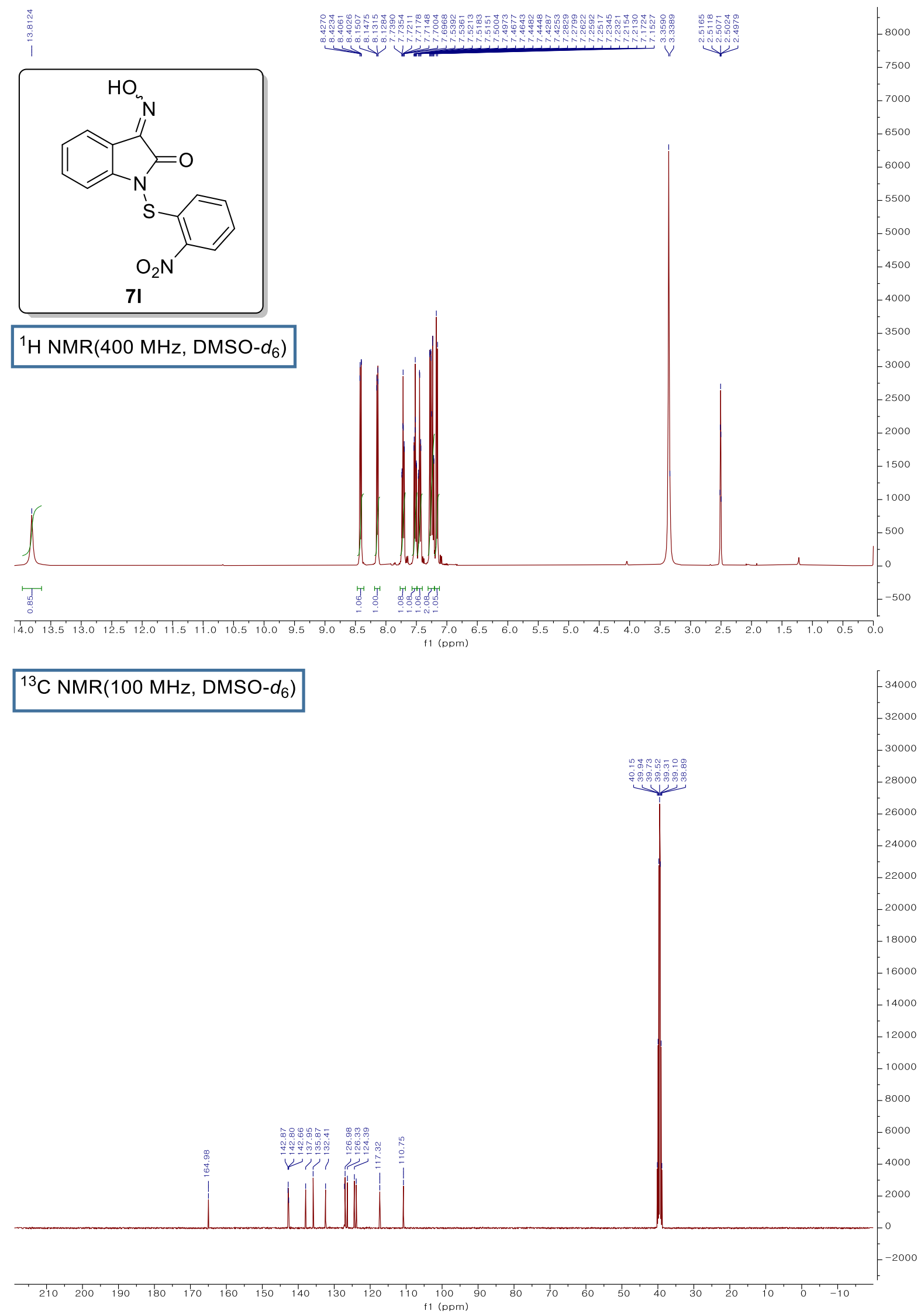


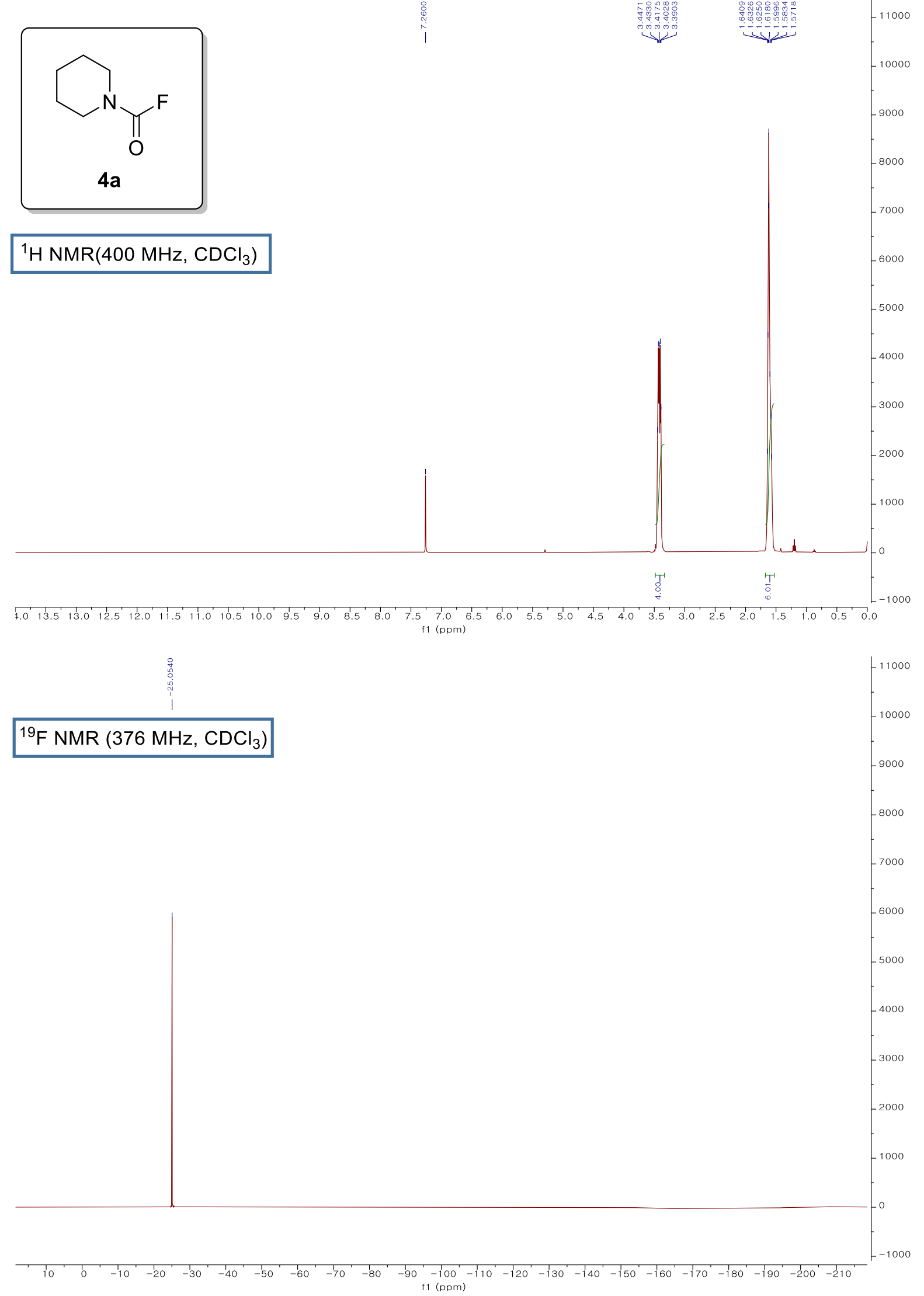




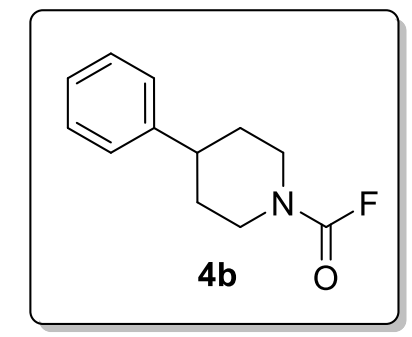

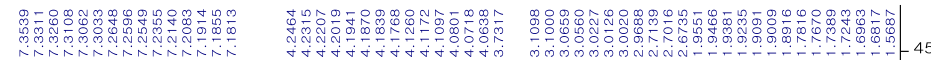

${ }^{1} \mathrm{H} \mathrm{NMR}\left(400 \mathrm{MHz}, \mathrm{CDCl}_{3}\right)$
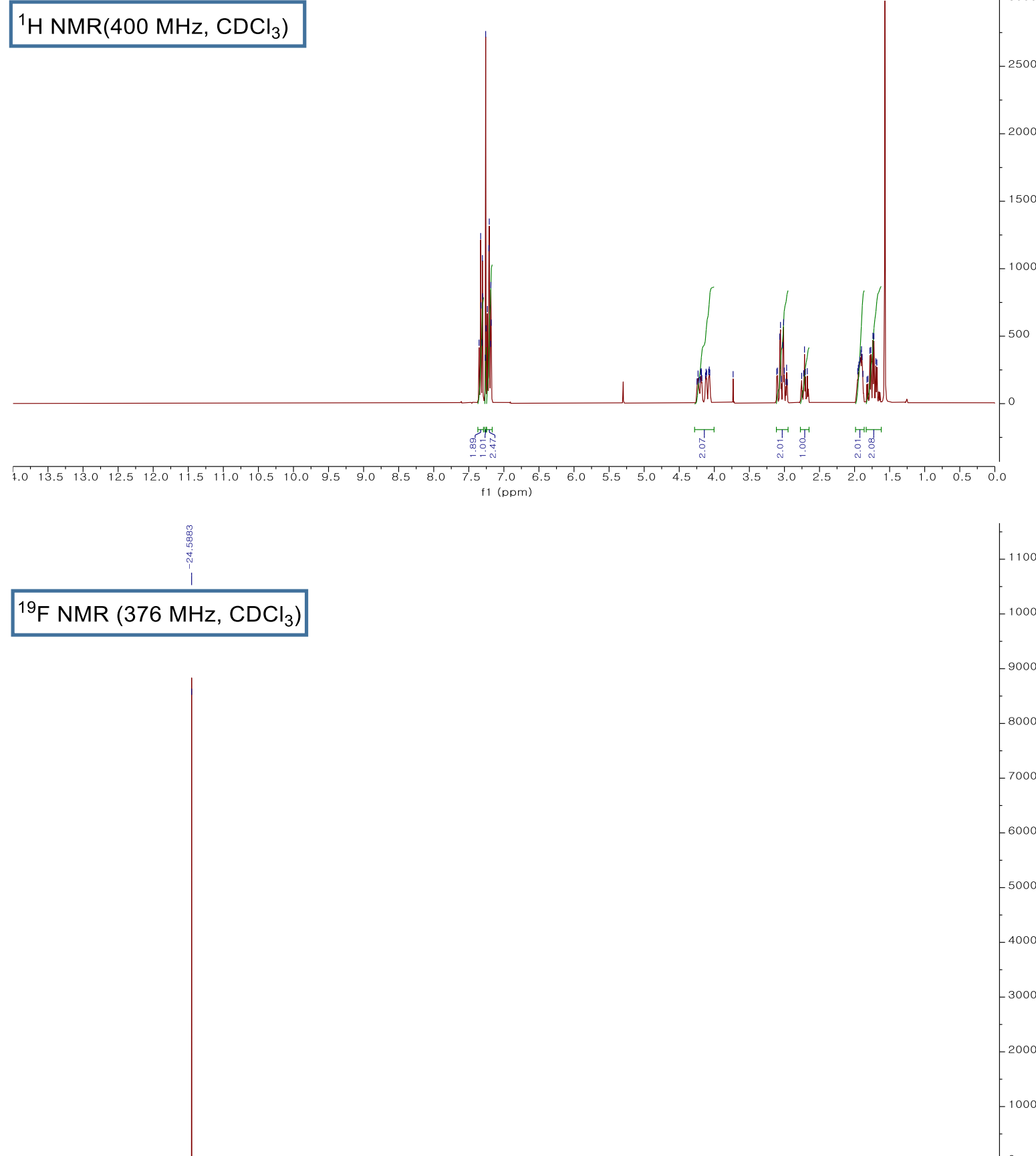

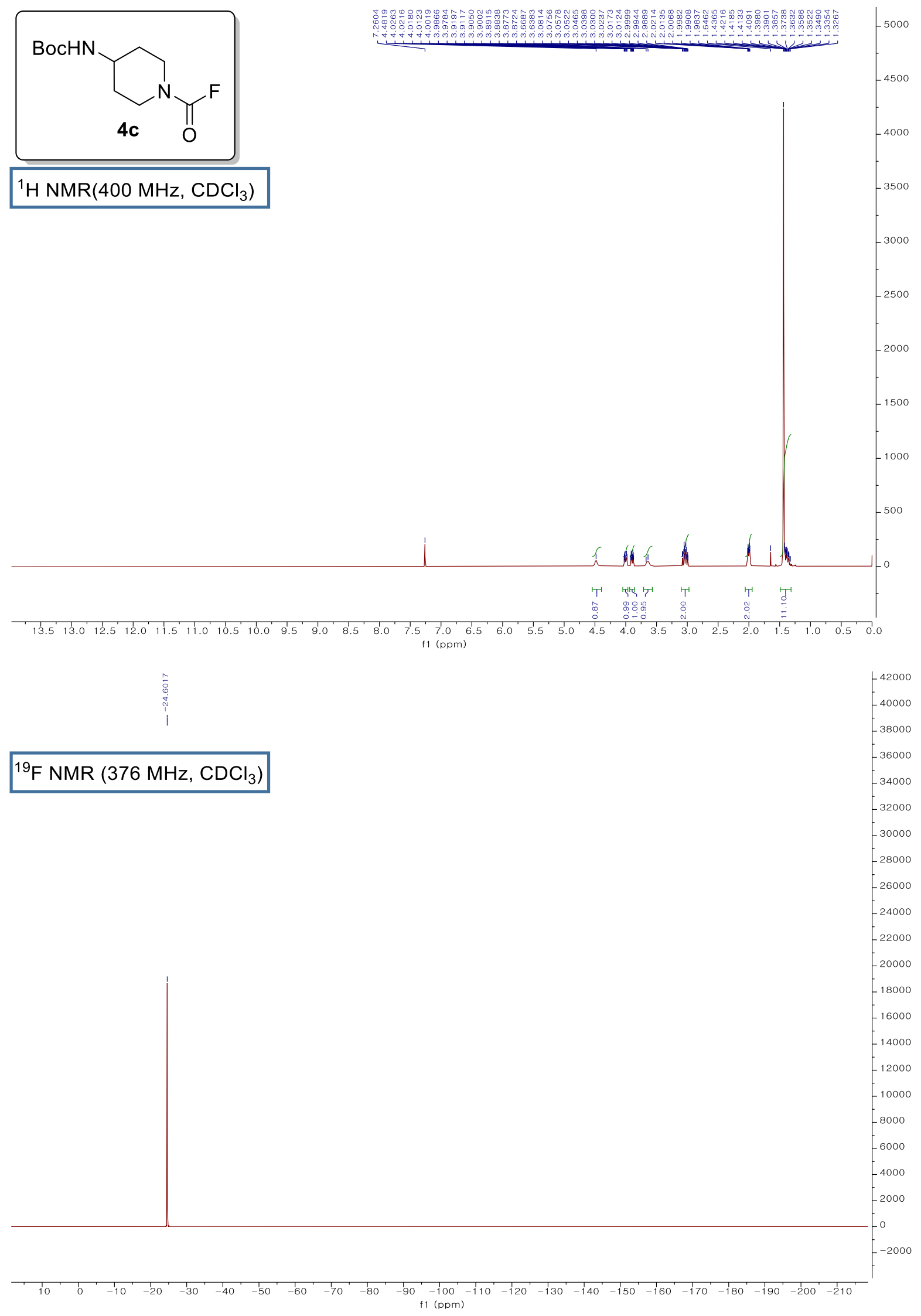

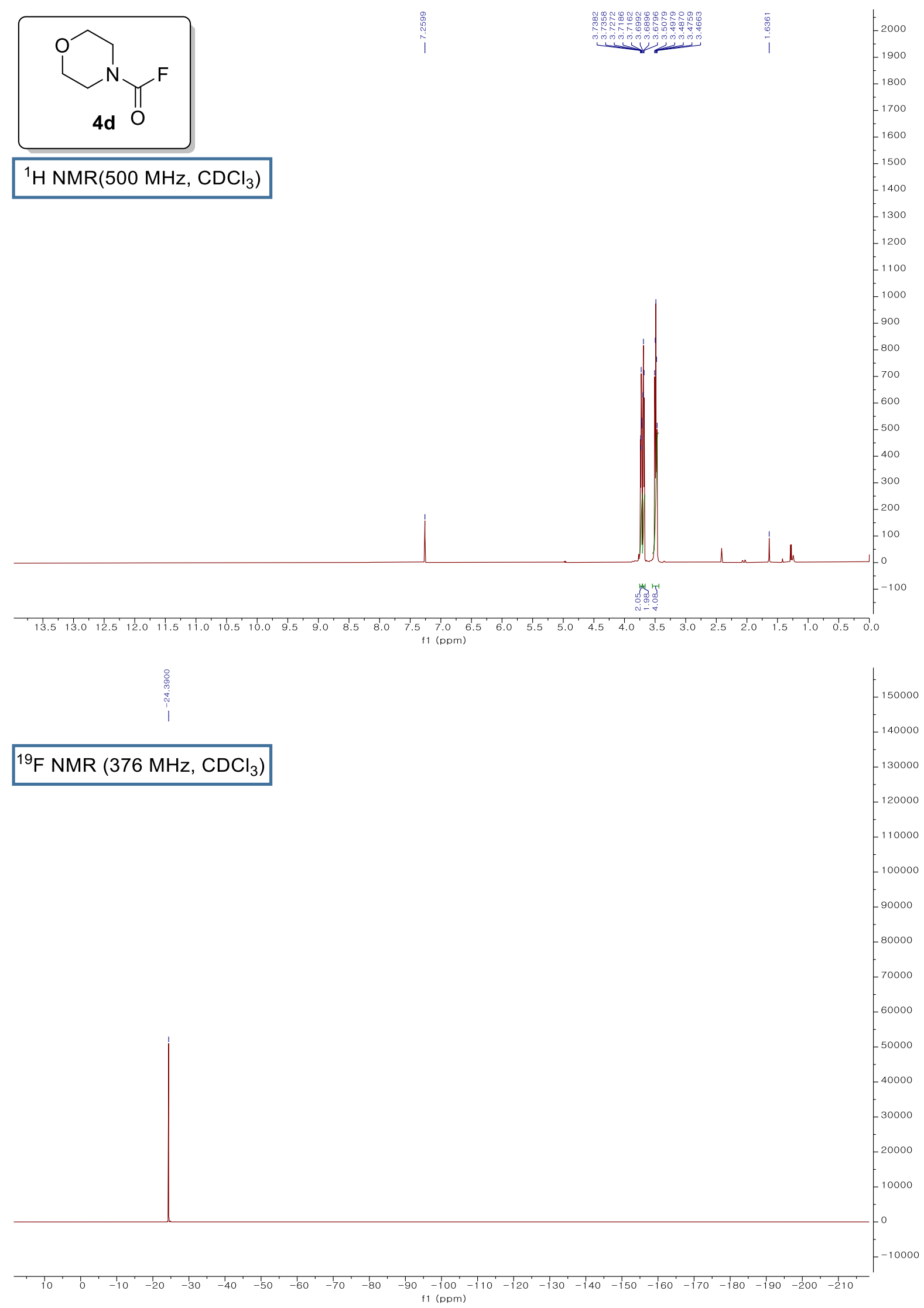


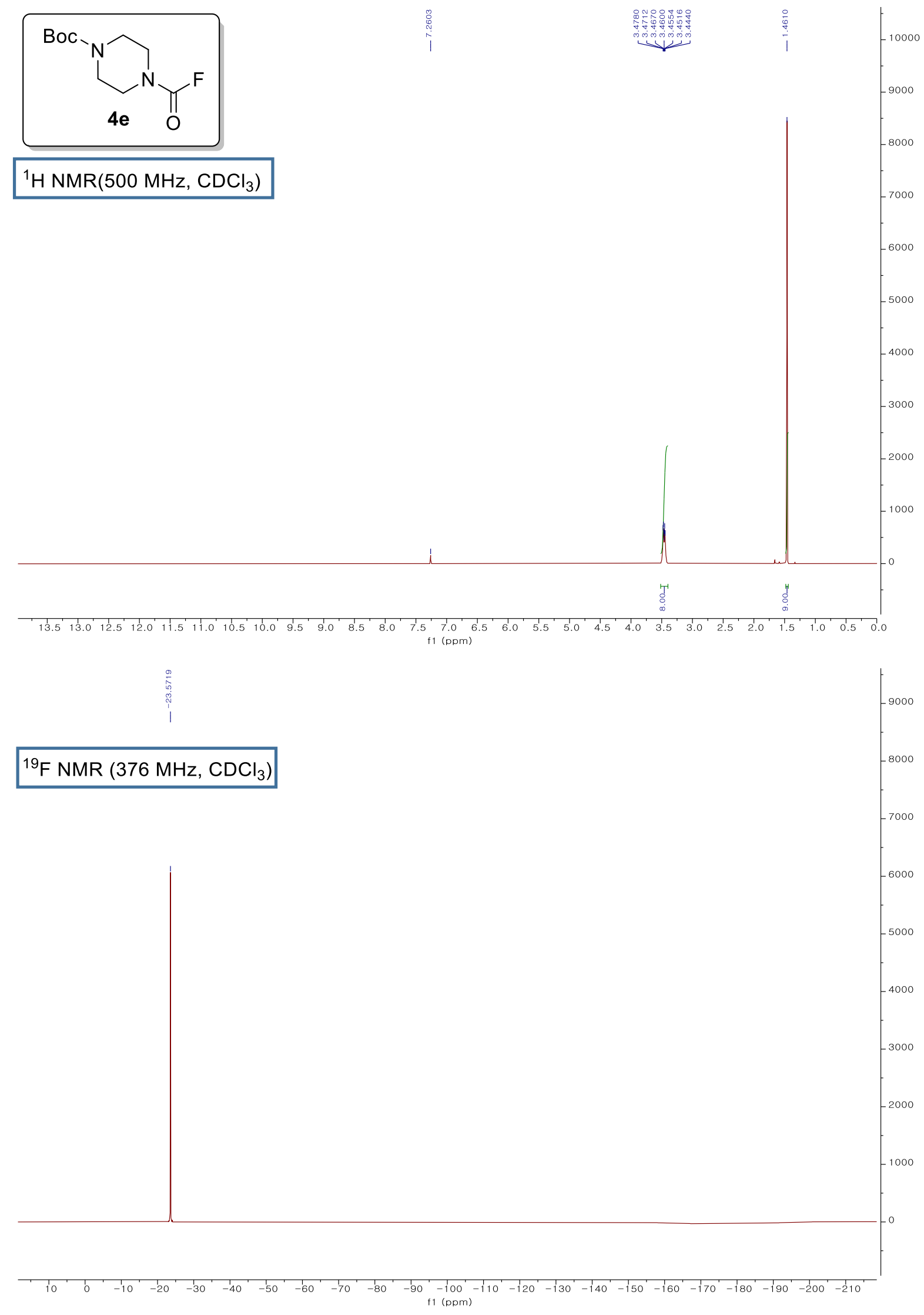




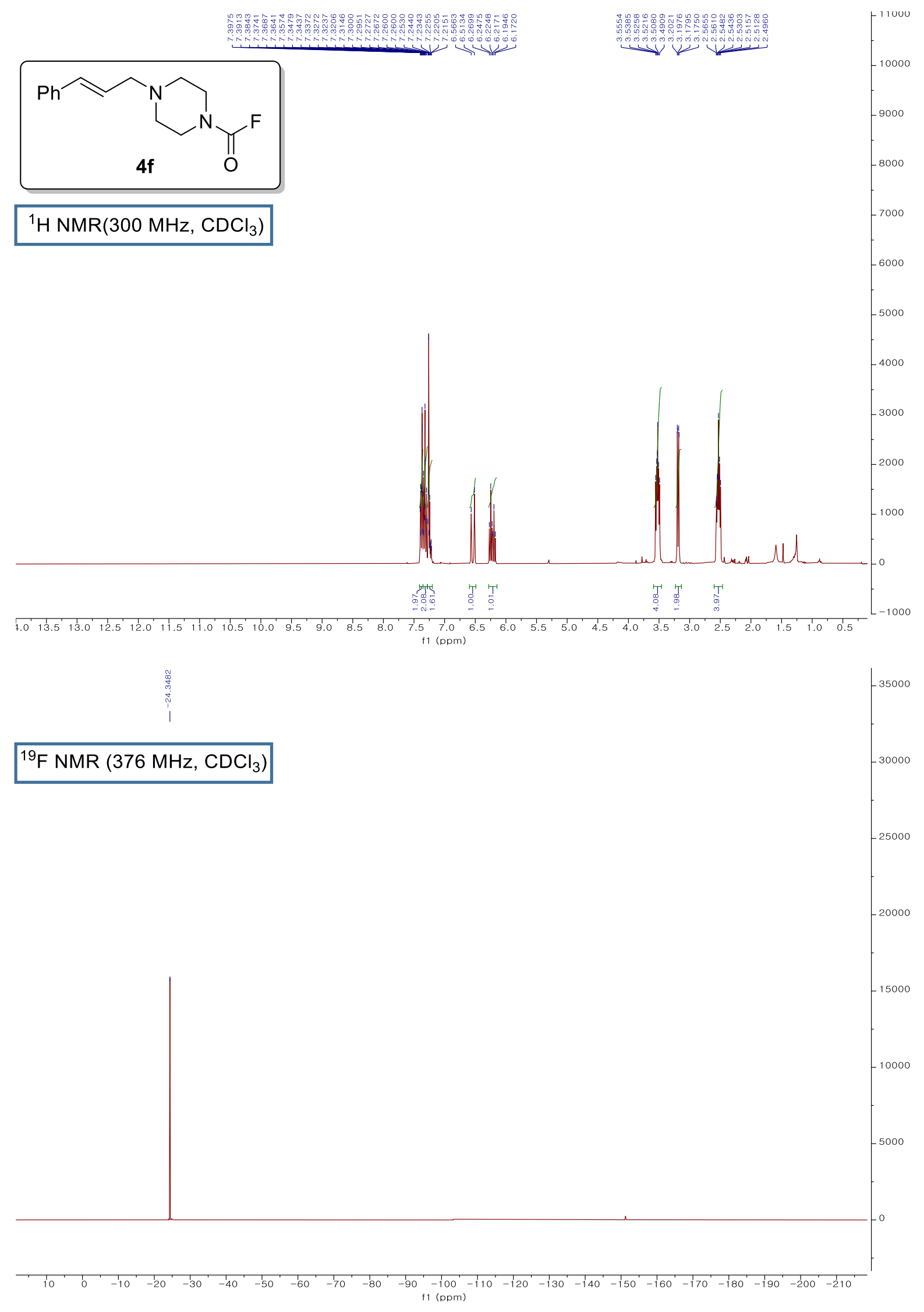



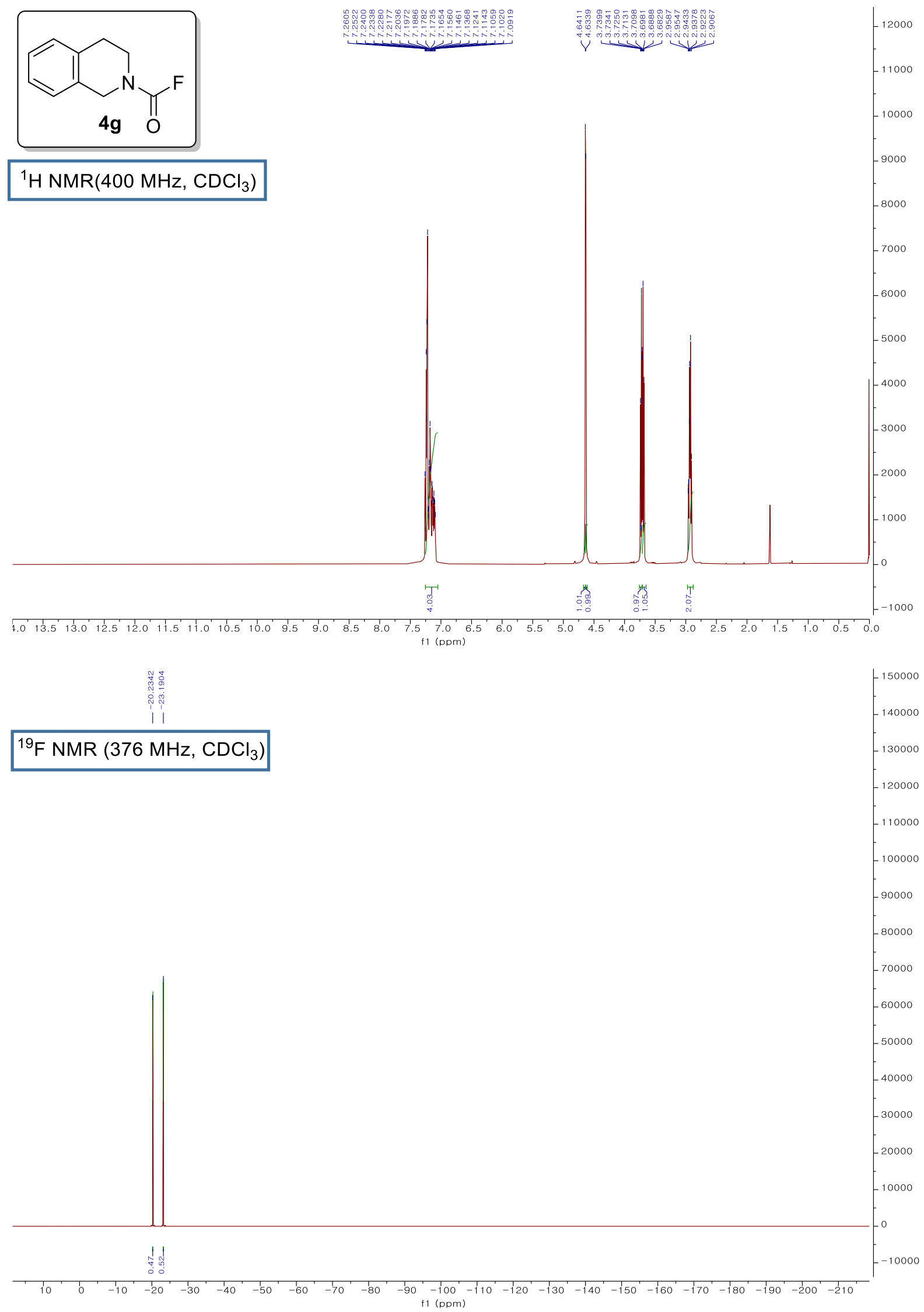


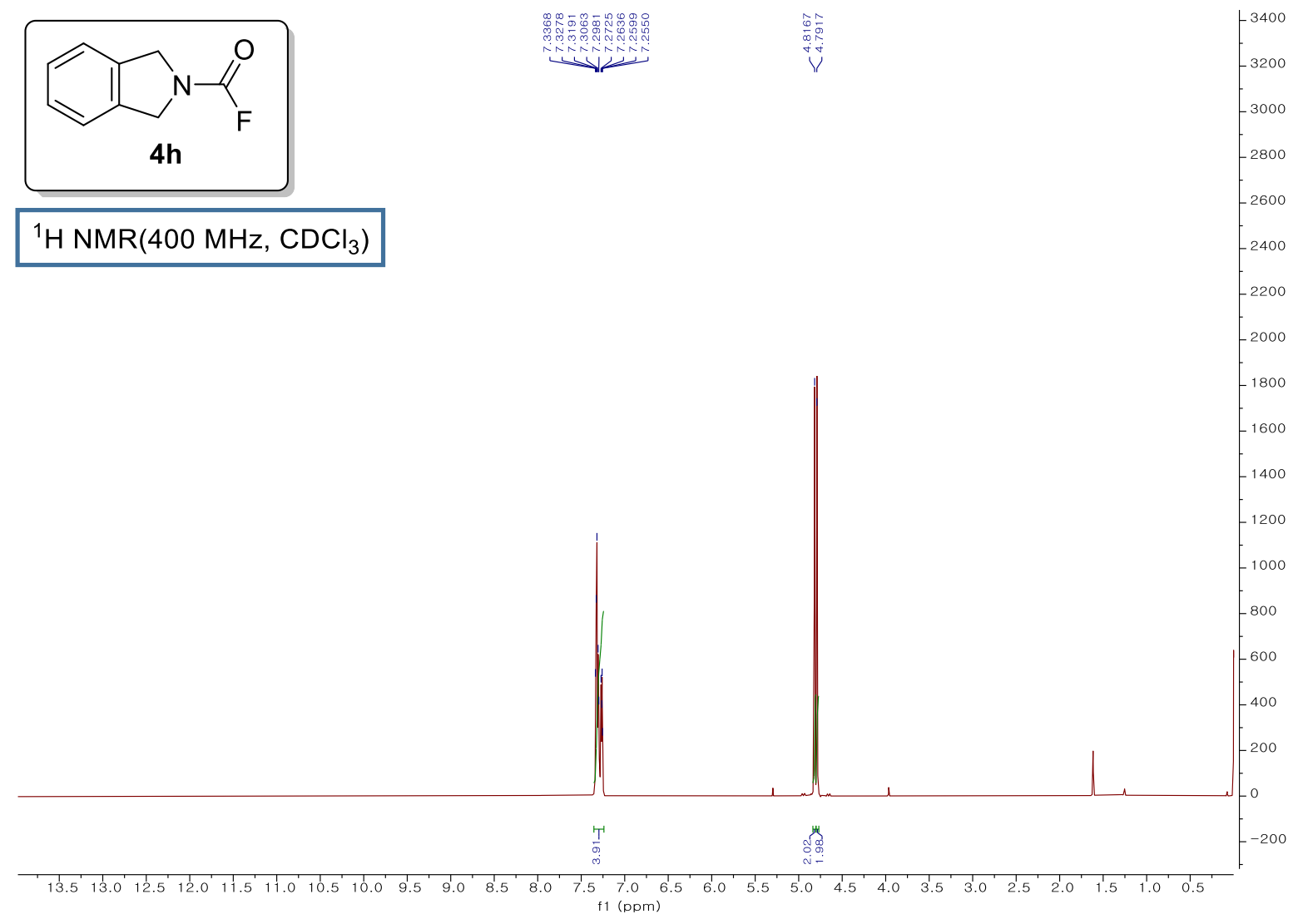

${ }^{13} \mathrm{C} \mathrm{NMR}\left(100 \mathrm{MHz}, \mathrm{CDCl}_{3}\right)$

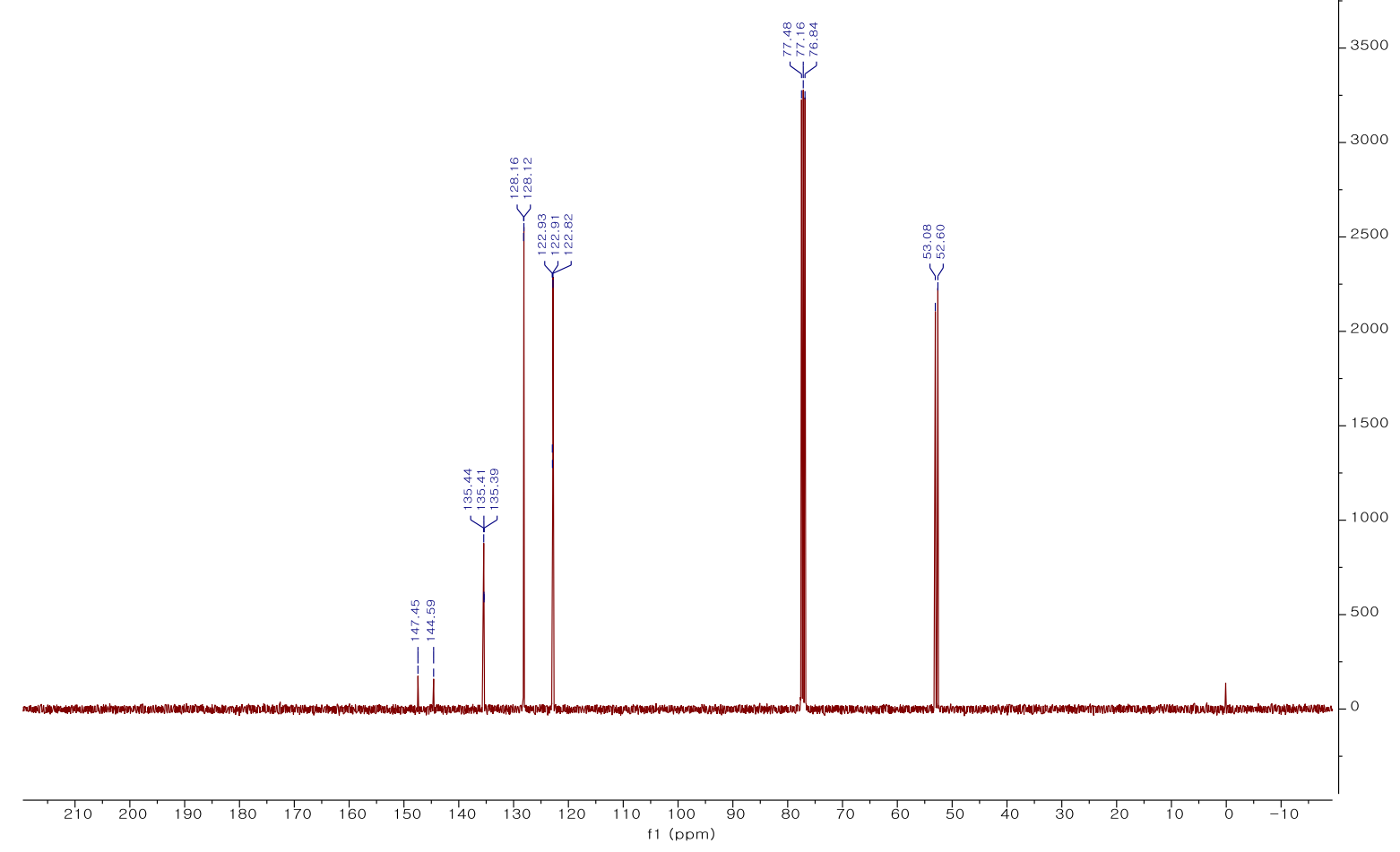



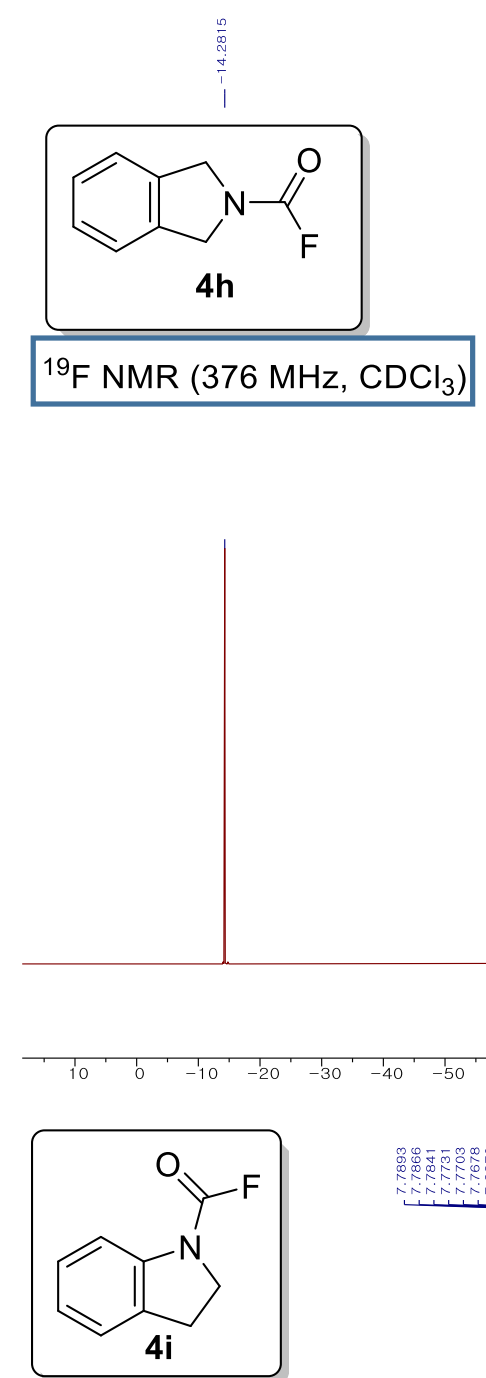

${ }^{1} \mathrm{H} \mathrm{NMR}\left(500 \mathrm{MHz}, \mathrm{CDCl}_{3}\right)$ 


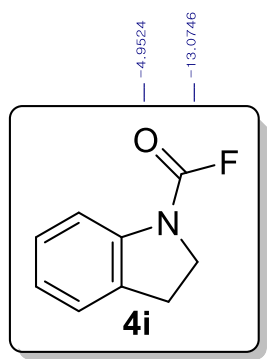

$\left.{ }^{19} \mathrm{~F} \mathrm{NMR} \mathrm{(376} \mathrm{MHz,} \mathrm{CDCl}_{3}\right)$
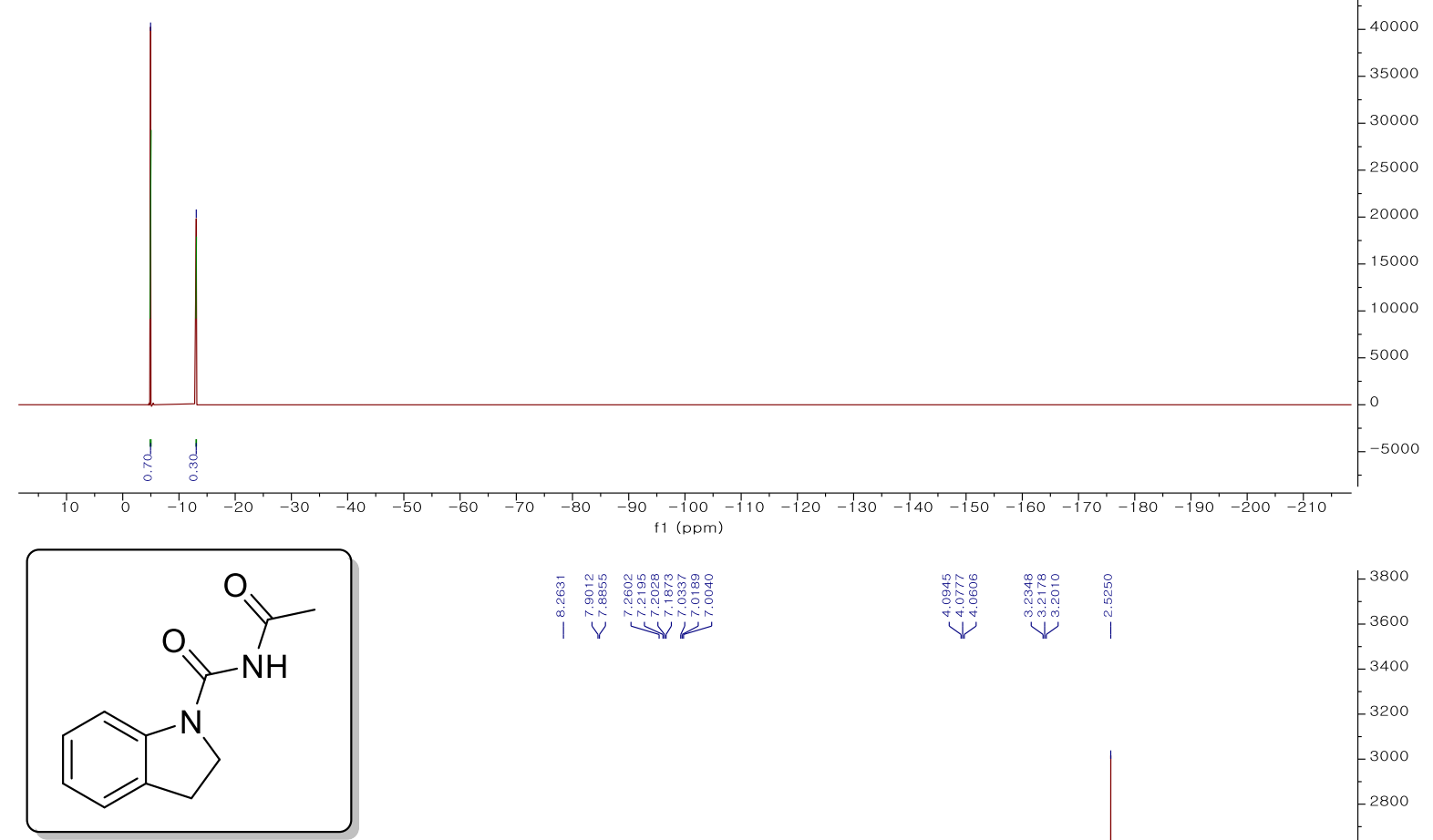

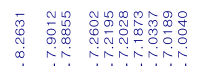

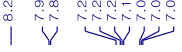

${ }^{1} \mathrm{H} \mathrm{NMR}\left(500 \mathrm{MHz}, \mathrm{CDCl}_{3}\right)$ 

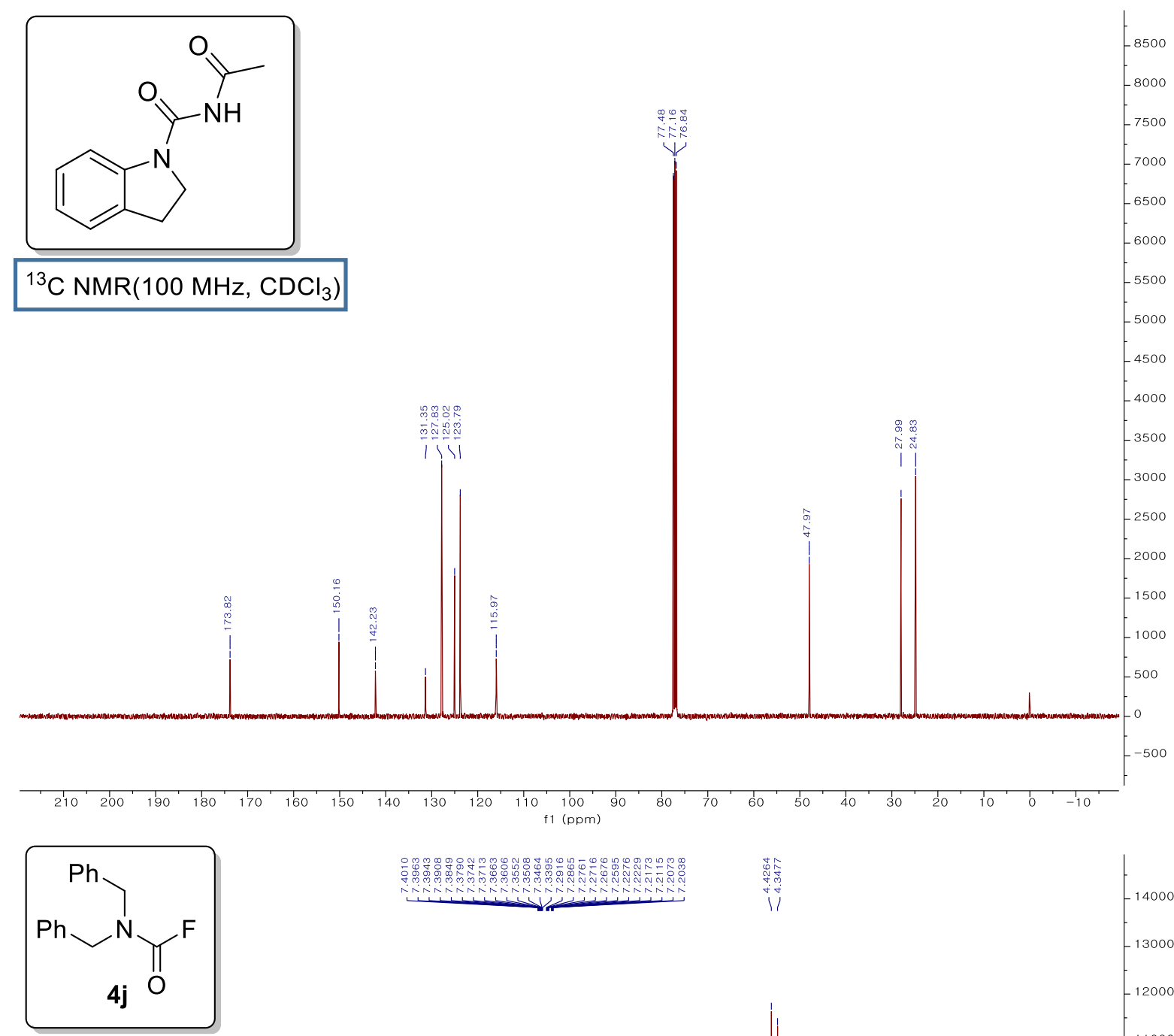

${ }^{1} \mathrm{H}$ NMR(400 MHz, $\left.\mathrm{CDCl}_{3}\right)$

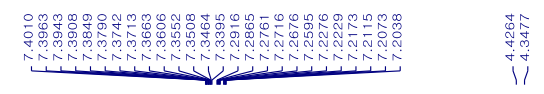

14000

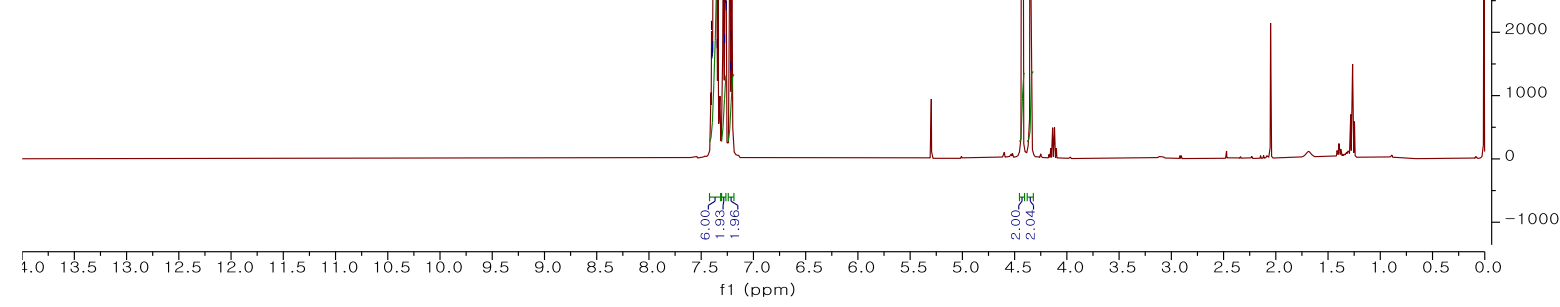



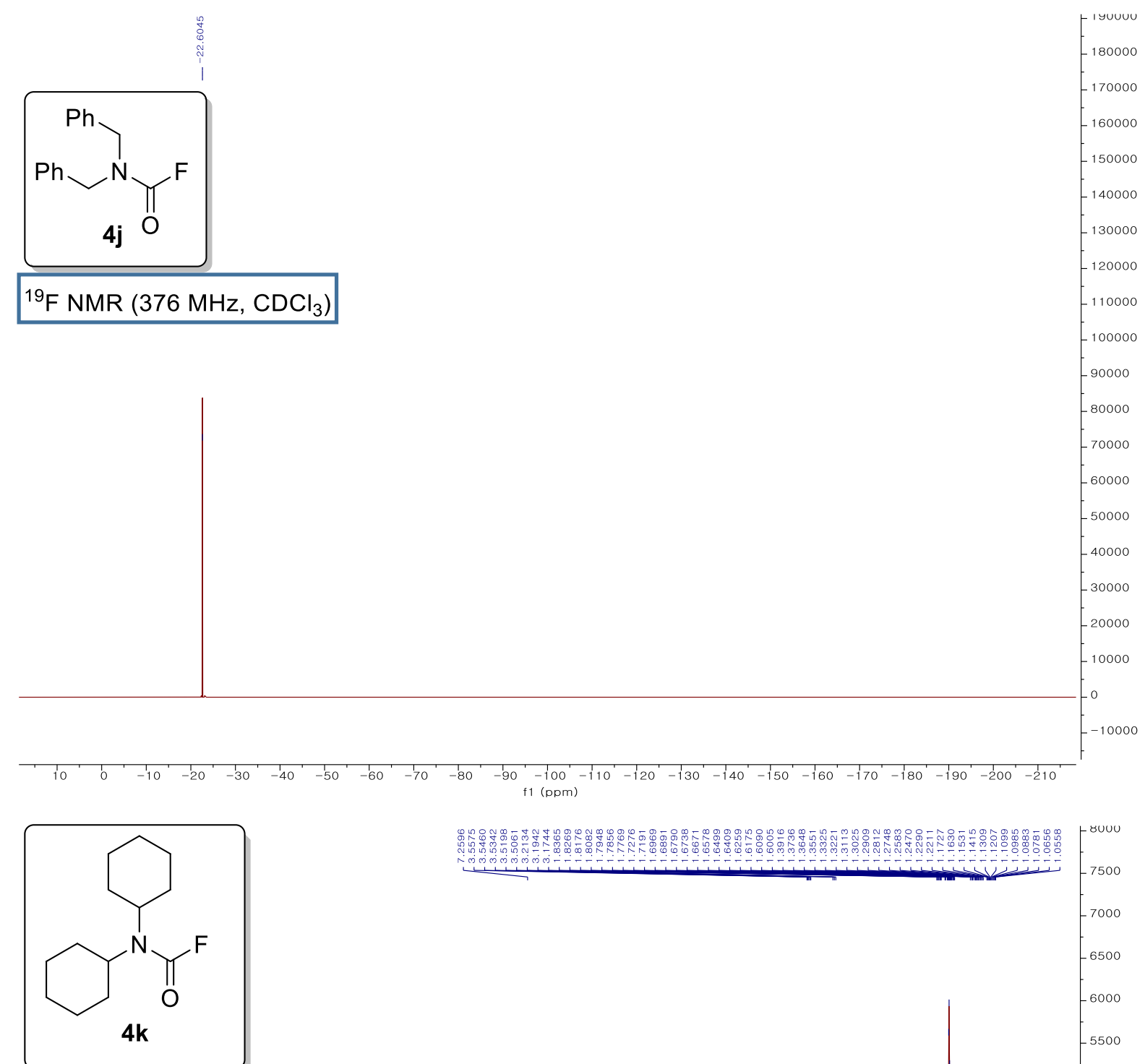

${ }^{1} \mathrm{H} \mathrm{NMR}\left(400 \mathrm{MHz}, \mathrm{CDCl}_{3}\right)$ 


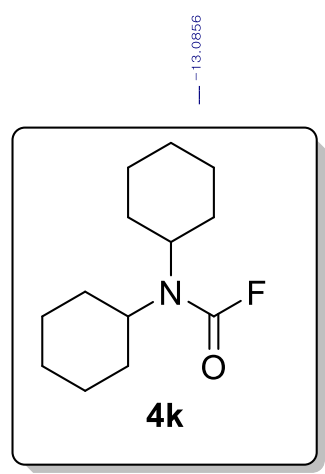

${ }^{19} \mathrm{~F} \mathrm{NMR}\left(376 \mathrm{MHz}, \mathrm{CDCl}_{3}\right)$

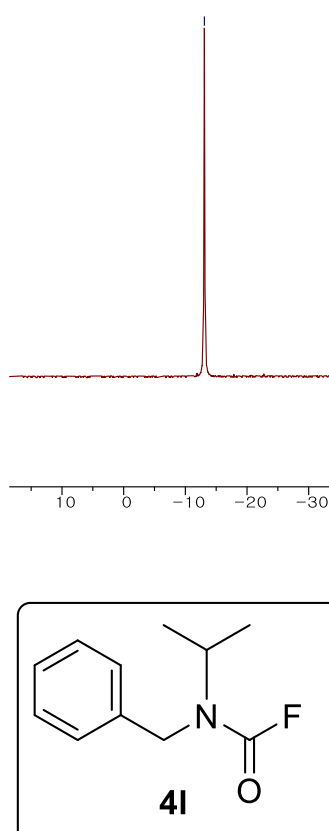

${ }^{1} \mathrm{H} \mathrm{NMR}\left(400 \mathrm{MHz}, \mathrm{CDCl}_{3}\right)$ f1 (ppm) 


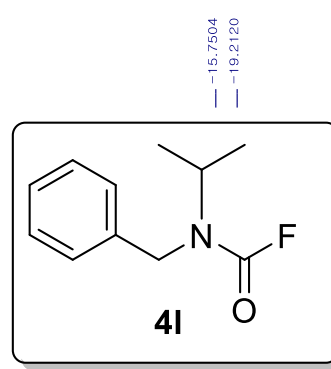

${ }^{19} \mathrm{~F}$ NMR (376 MHz, $\mathrm{CDCl}_{3}$ )
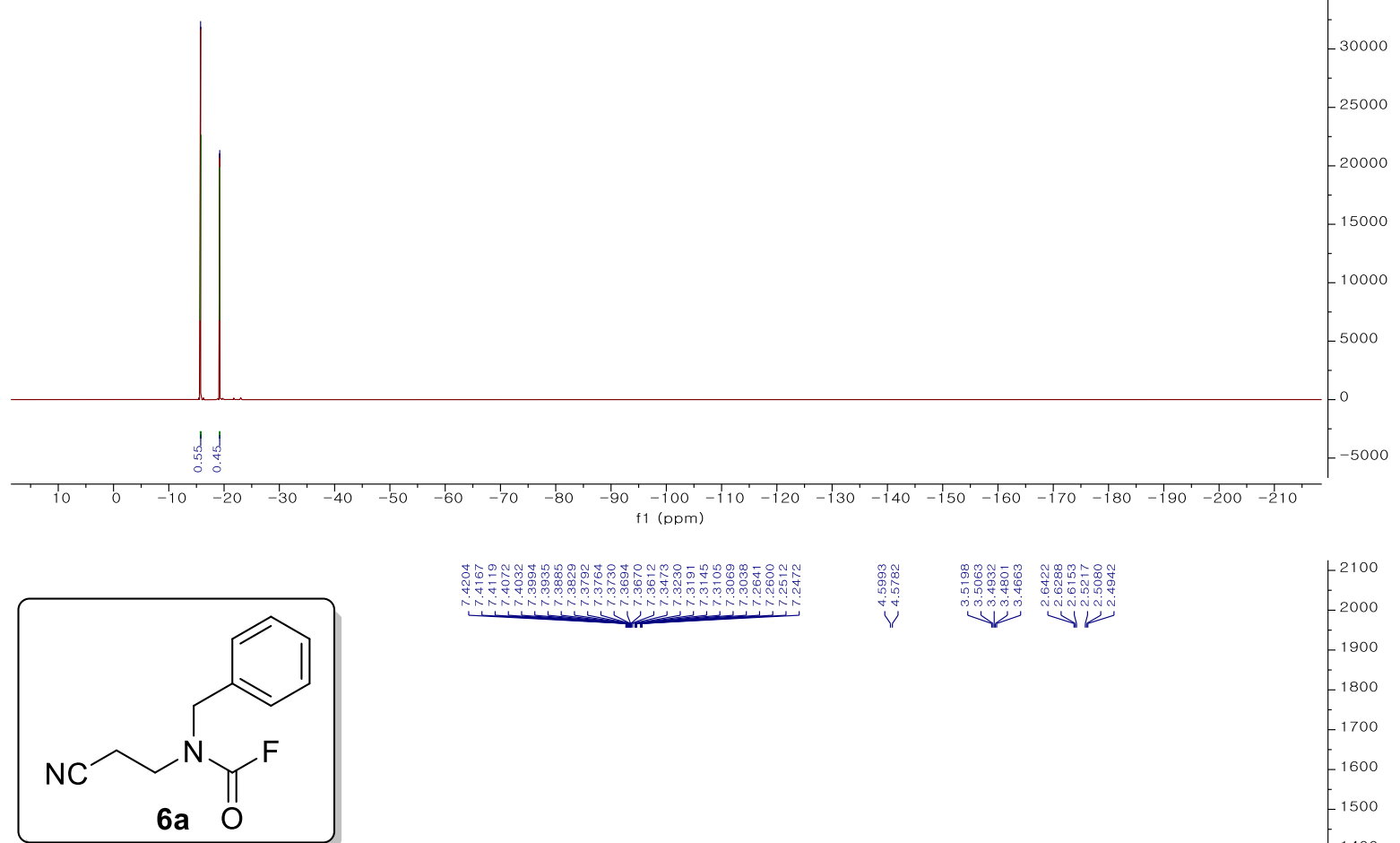

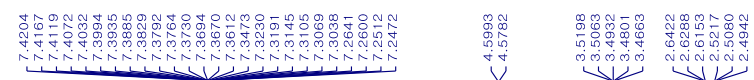

$-2100$

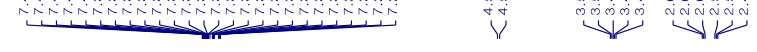

-2000
-1900

${ }^{1} \mathrm{H} \mathrm{NMR}\left(500 \mathrm{MHz}, \mathrm{CDCl}_{3}\right)$

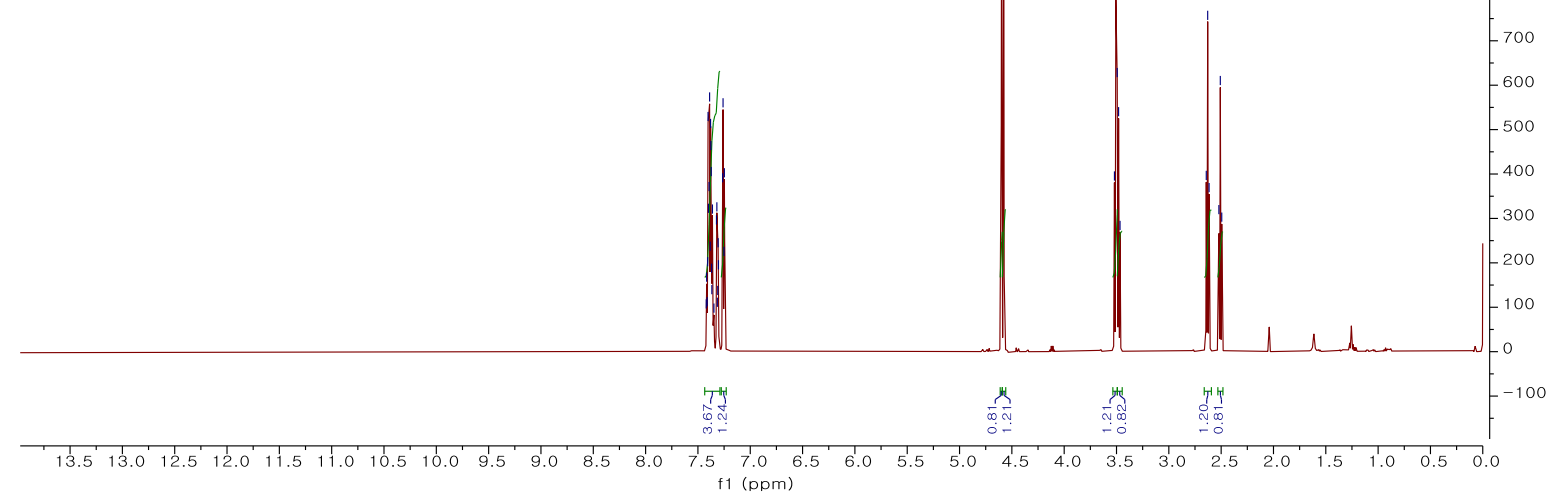



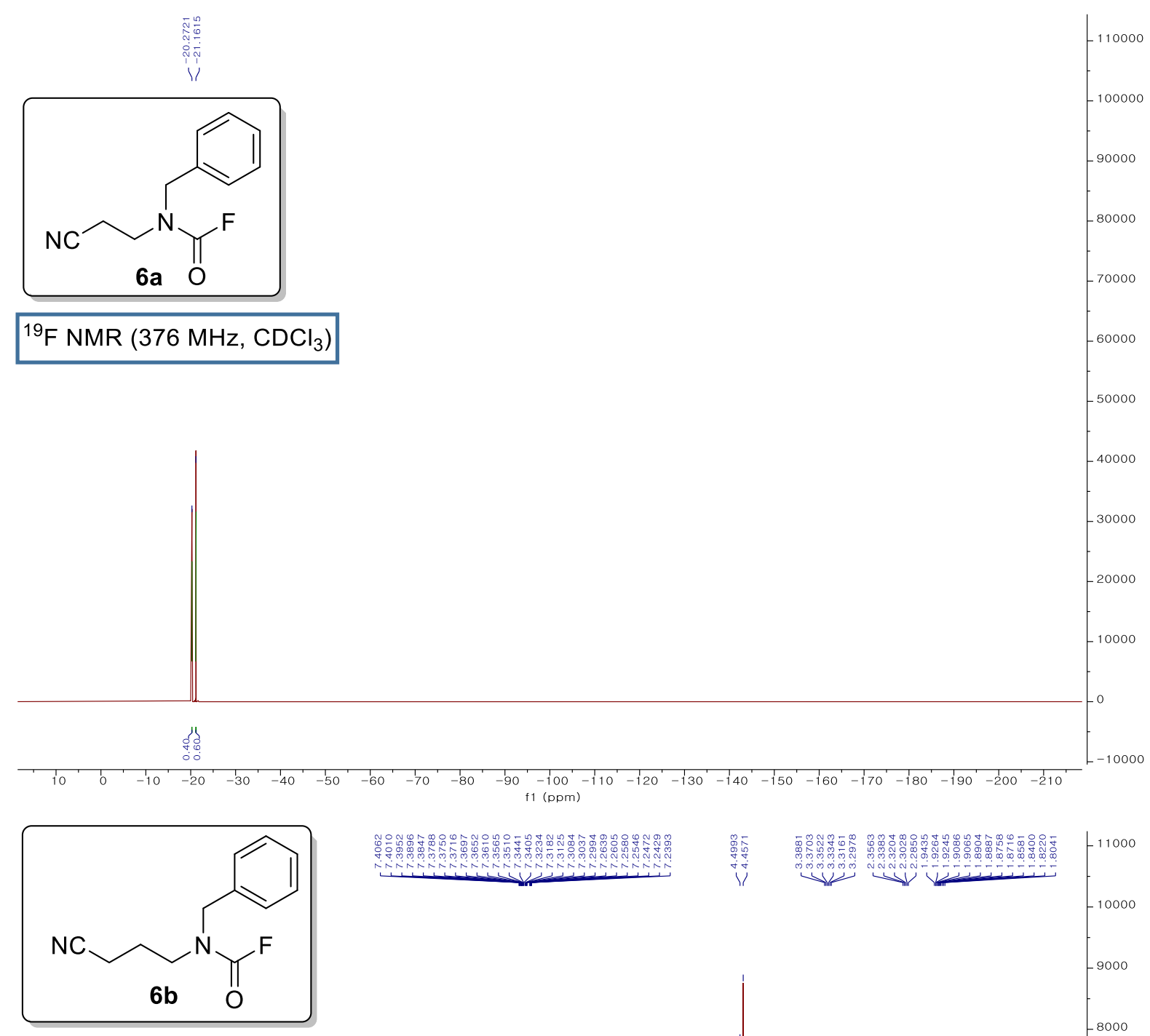

${ }^{1} \mathrm{H}$ NMR(400 MHz, $\left.\mathrm{CDCl}_{3}\right)$

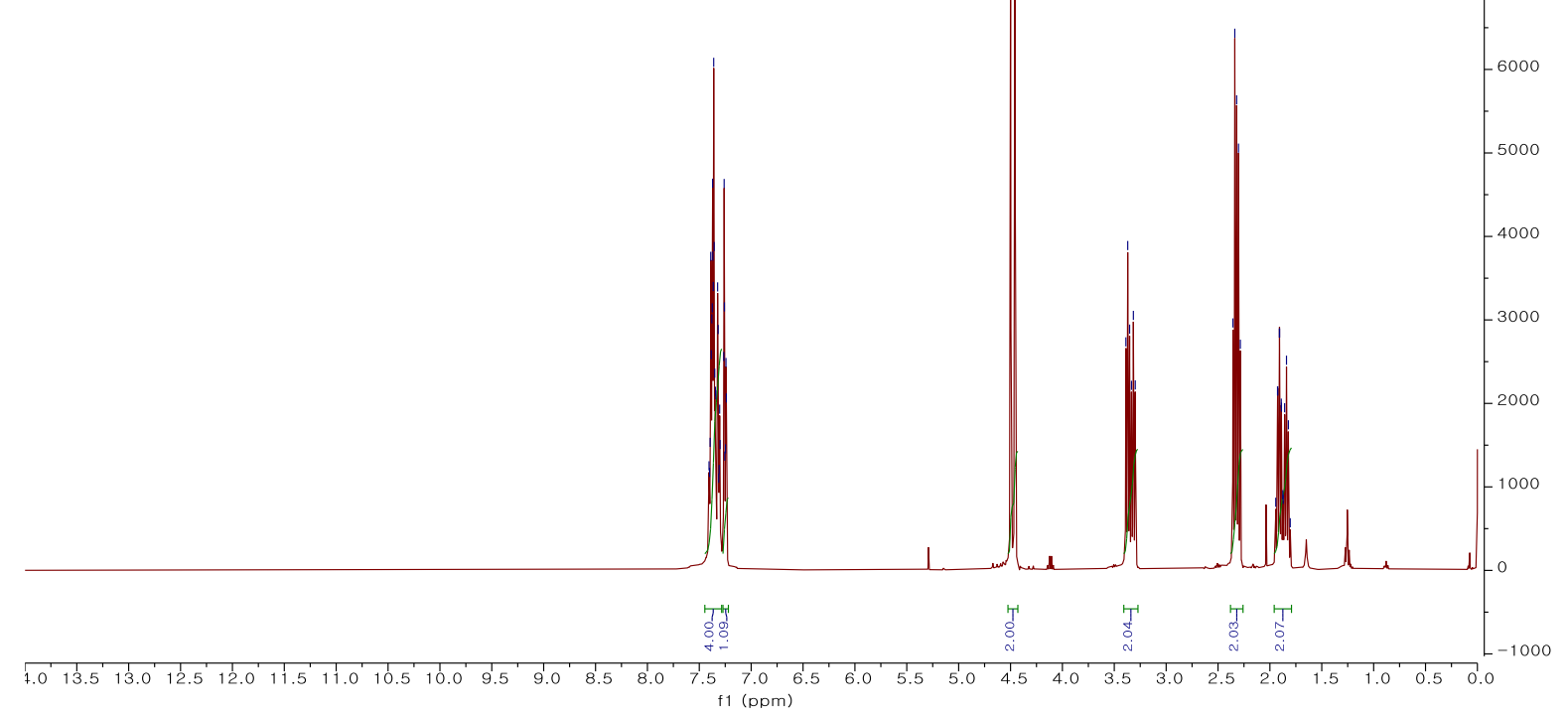



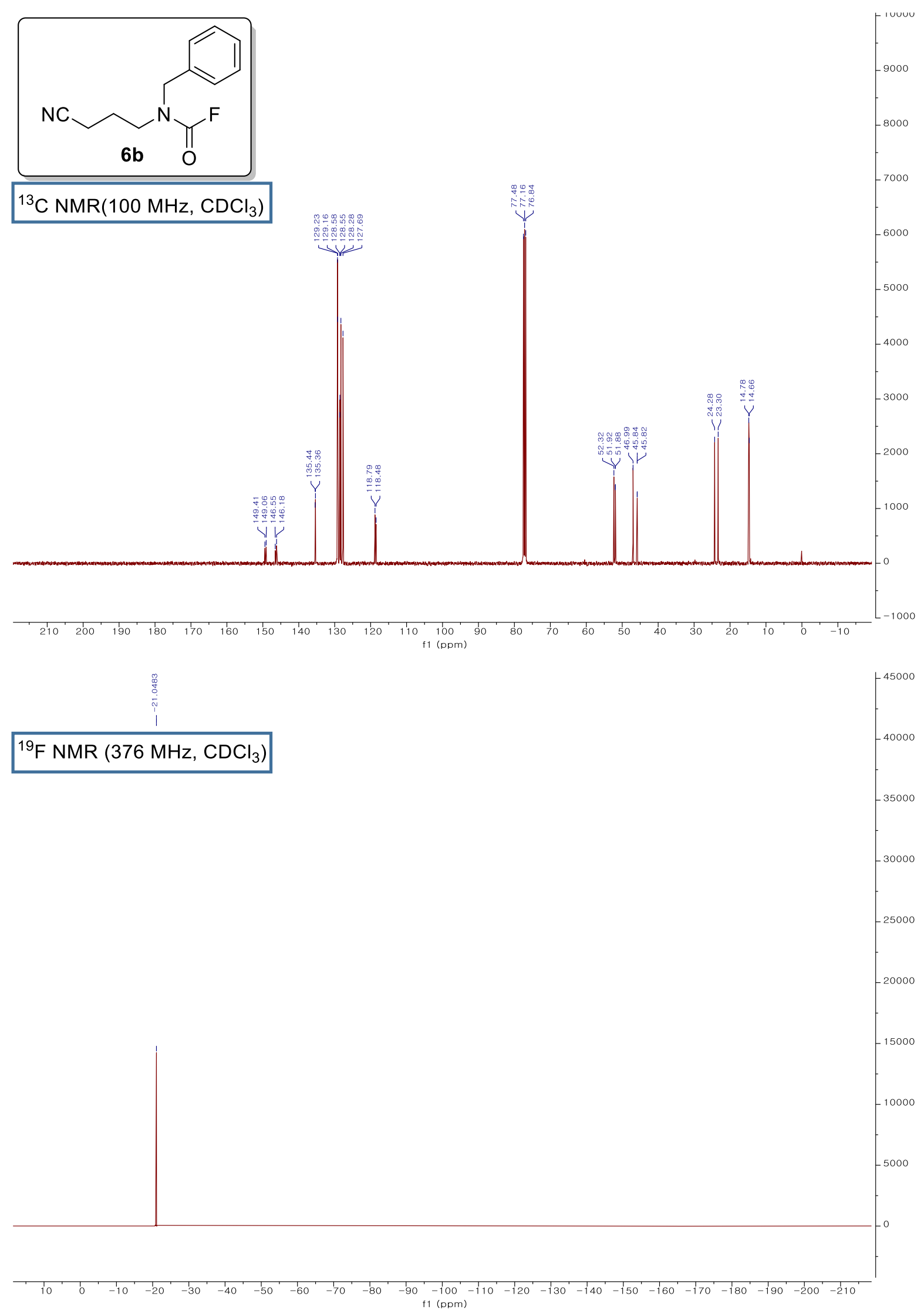

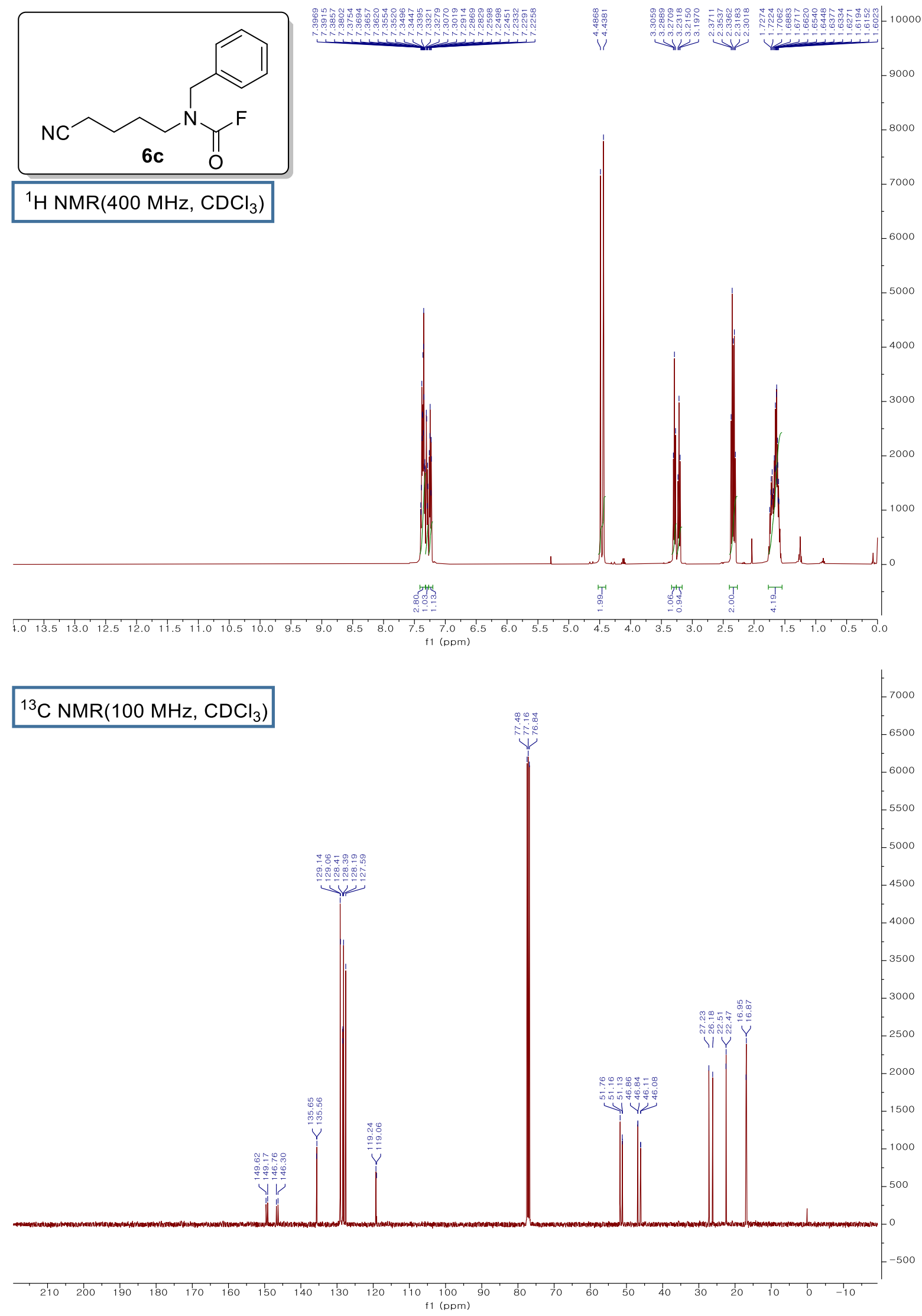

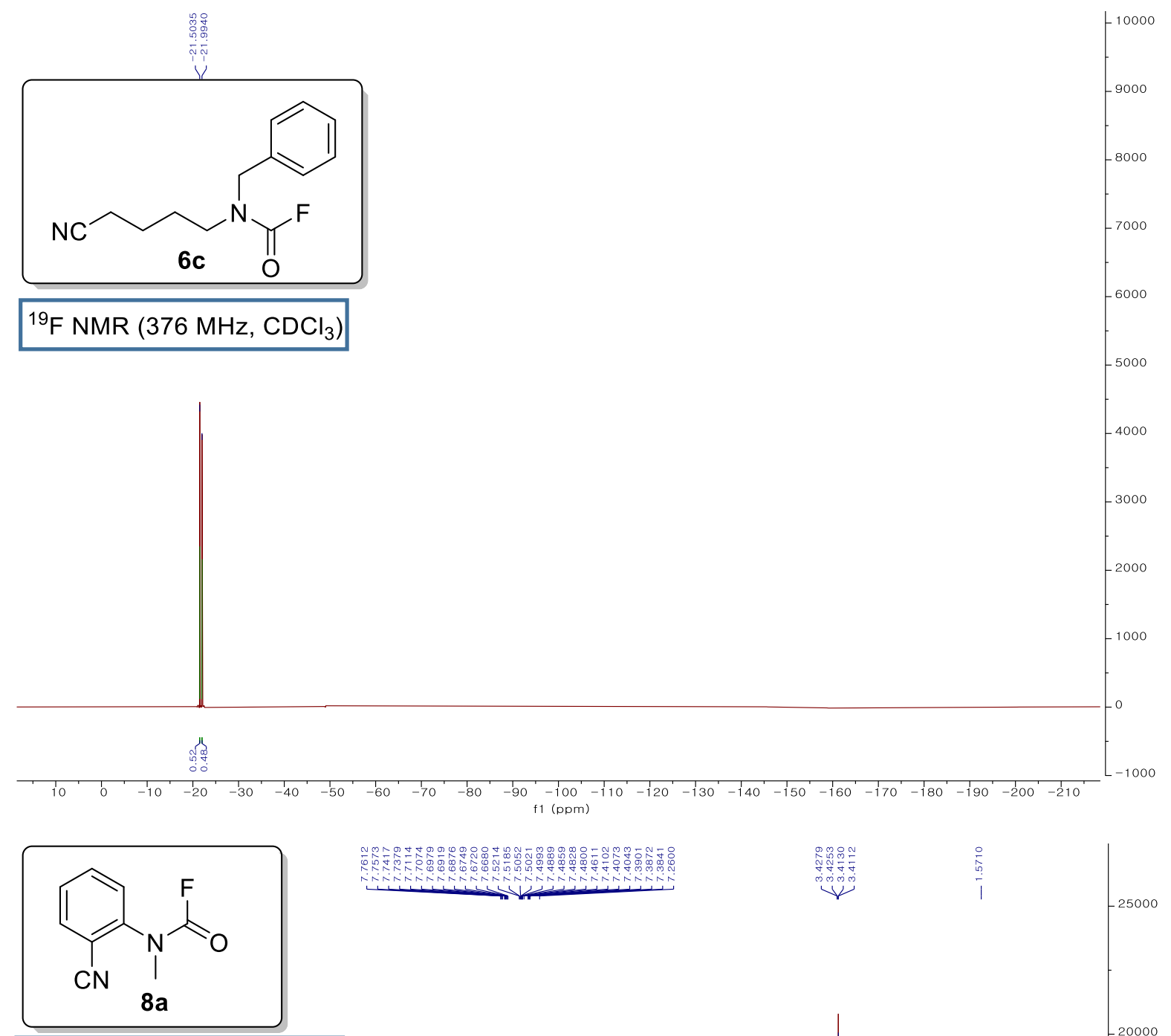

${ }^{1} \mathrm{H}$ NMR(400 MHz, $\mathrm{CDCl}_{3}$ )

${ }^{19} \mathrm{~F}$ NMR (376 MHz, CDCl 3 ) 

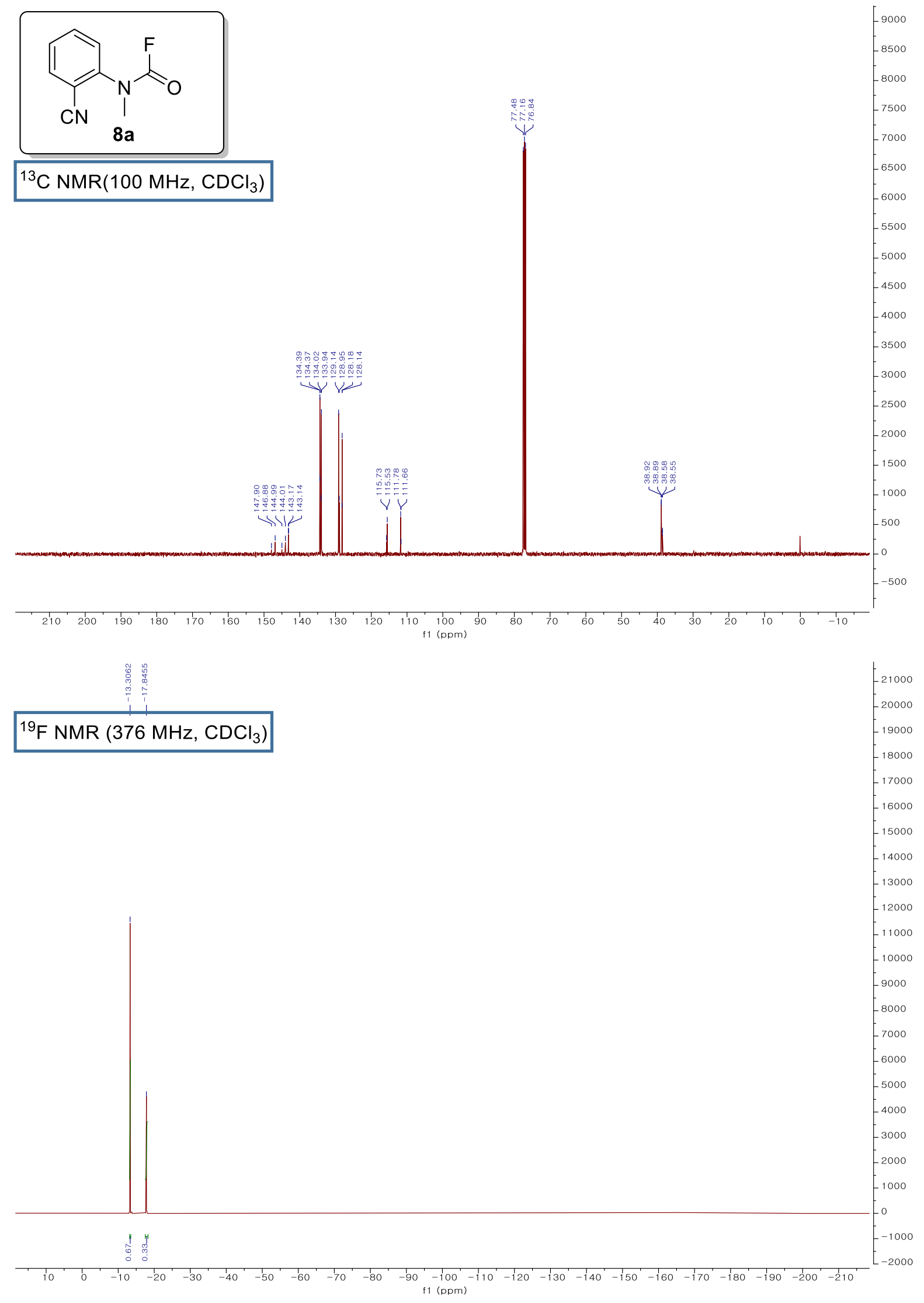


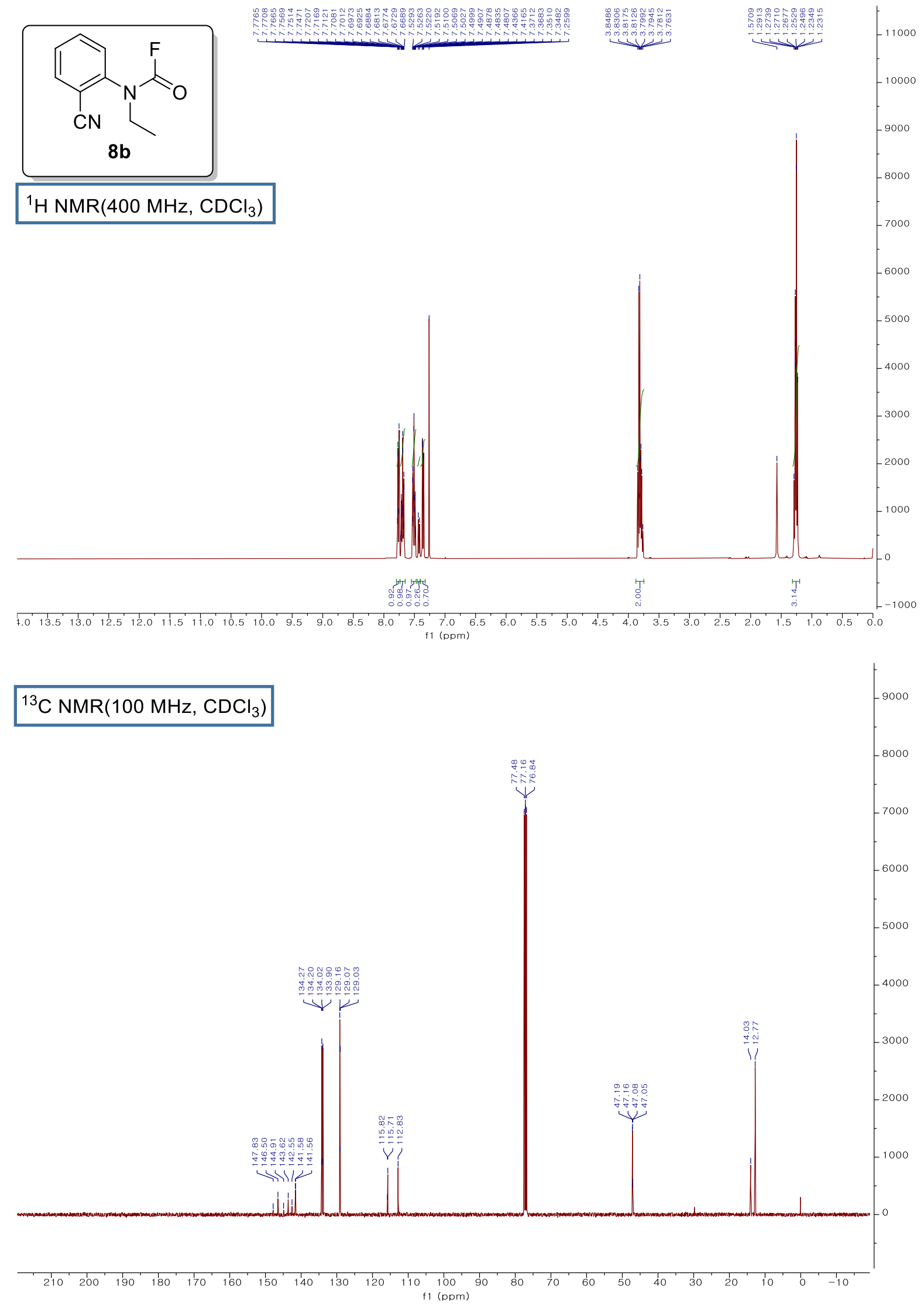




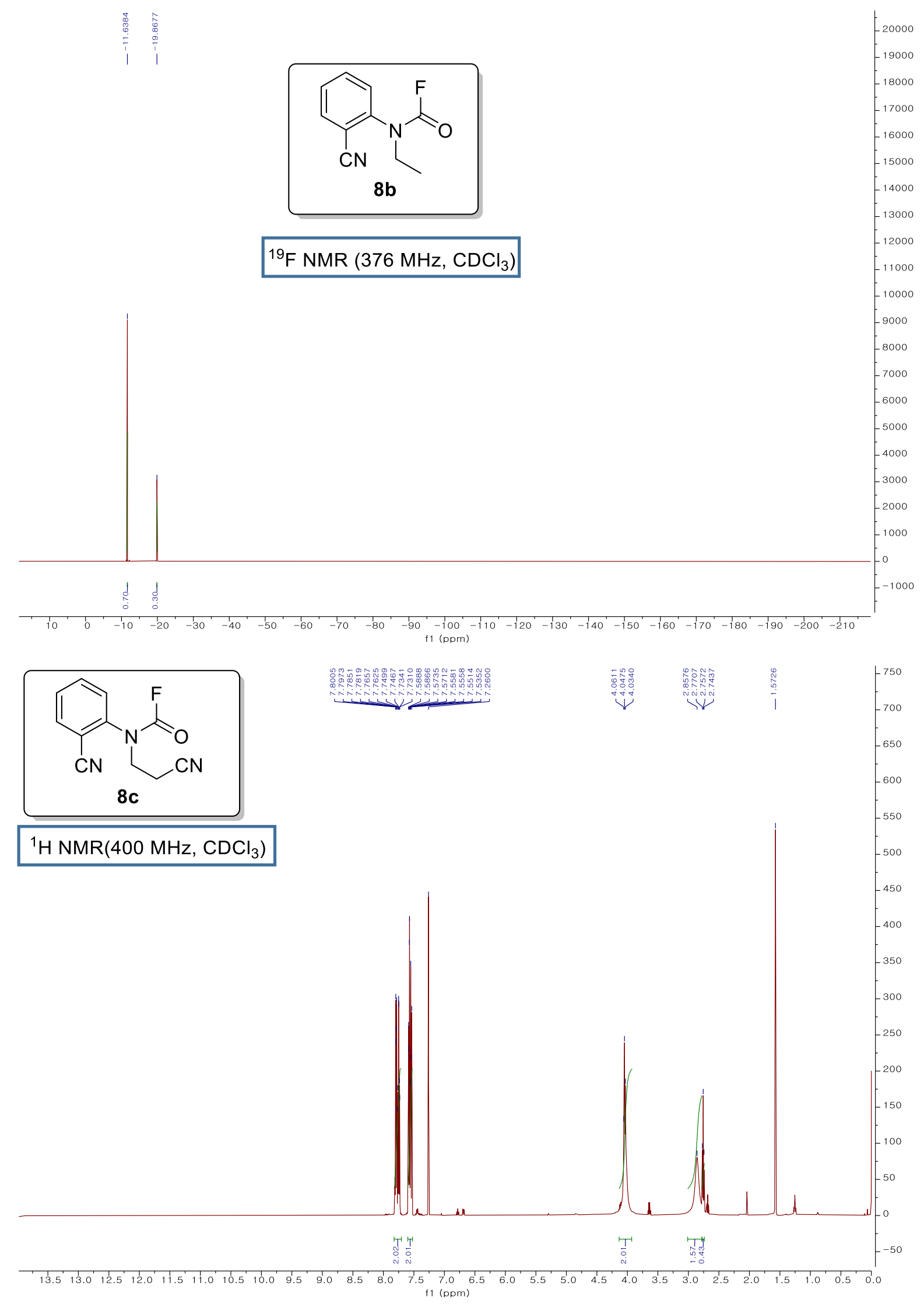




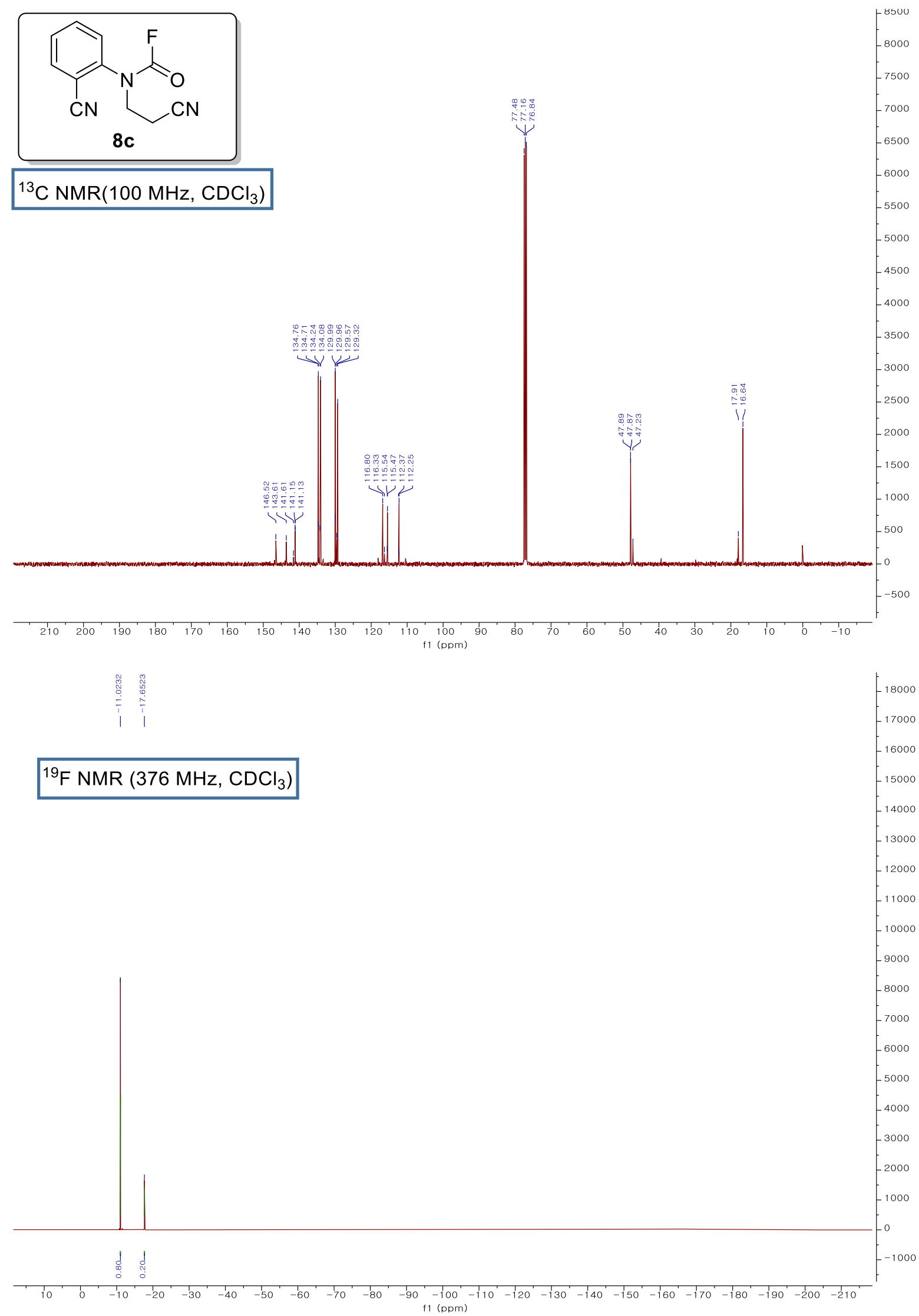



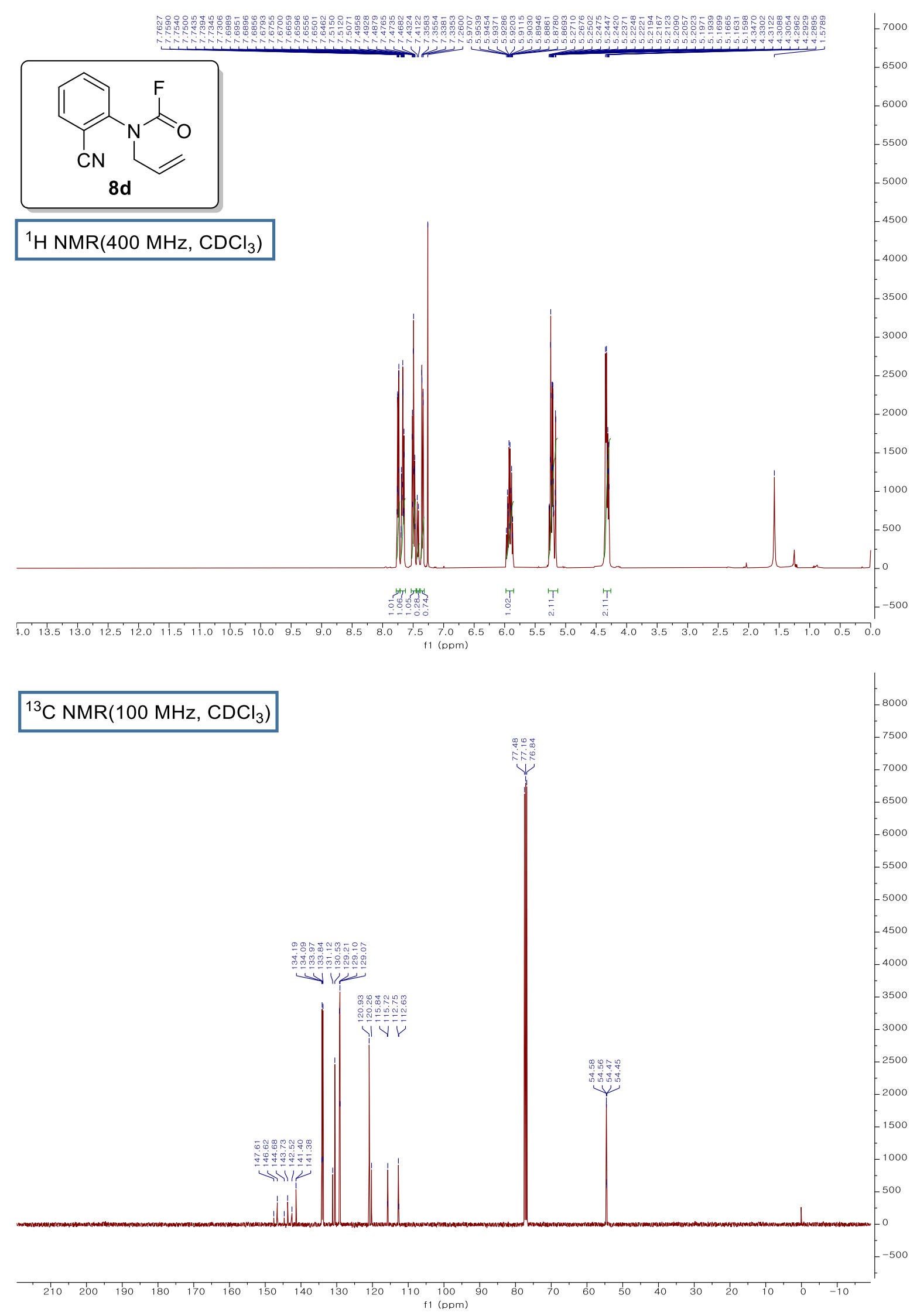


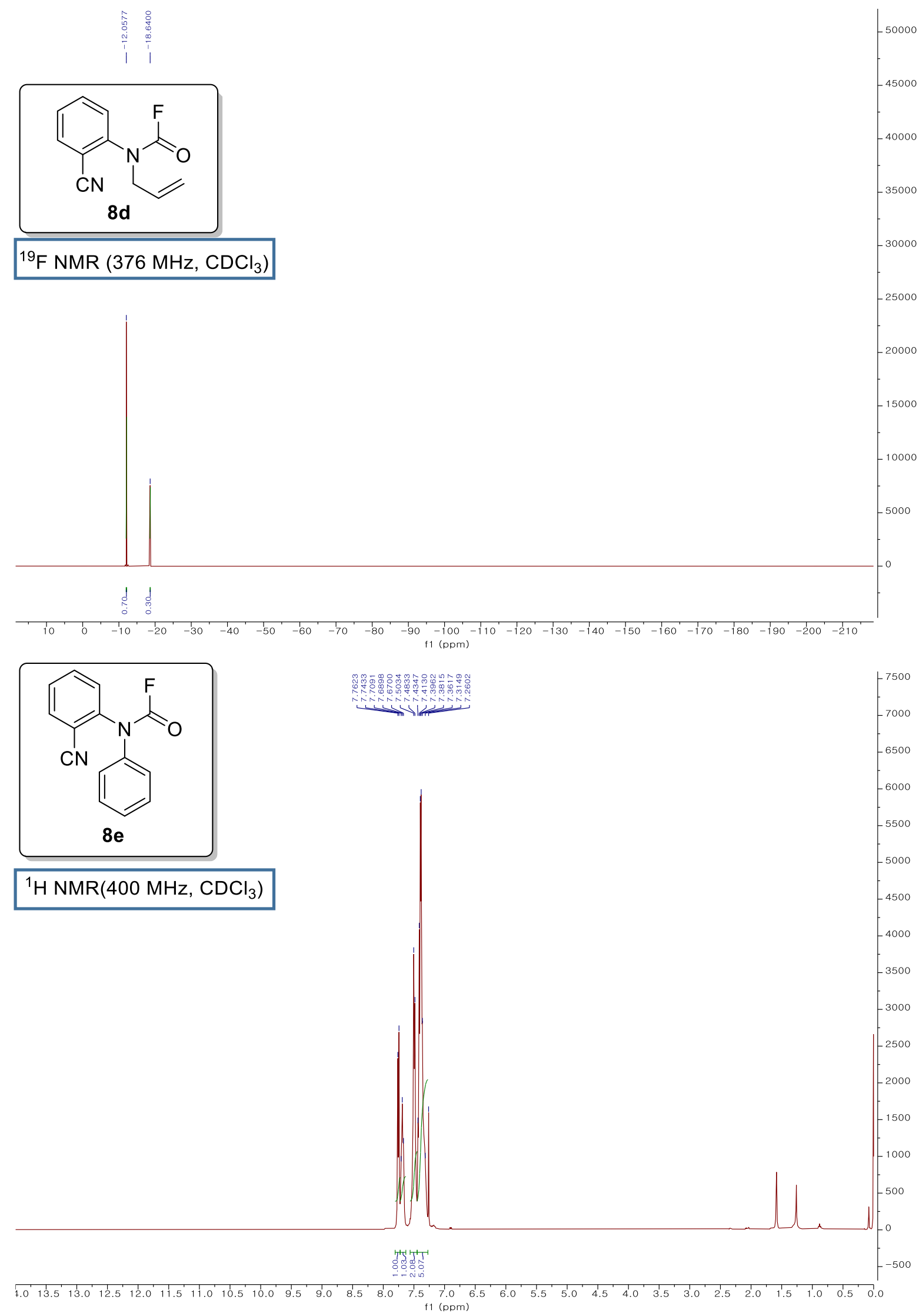




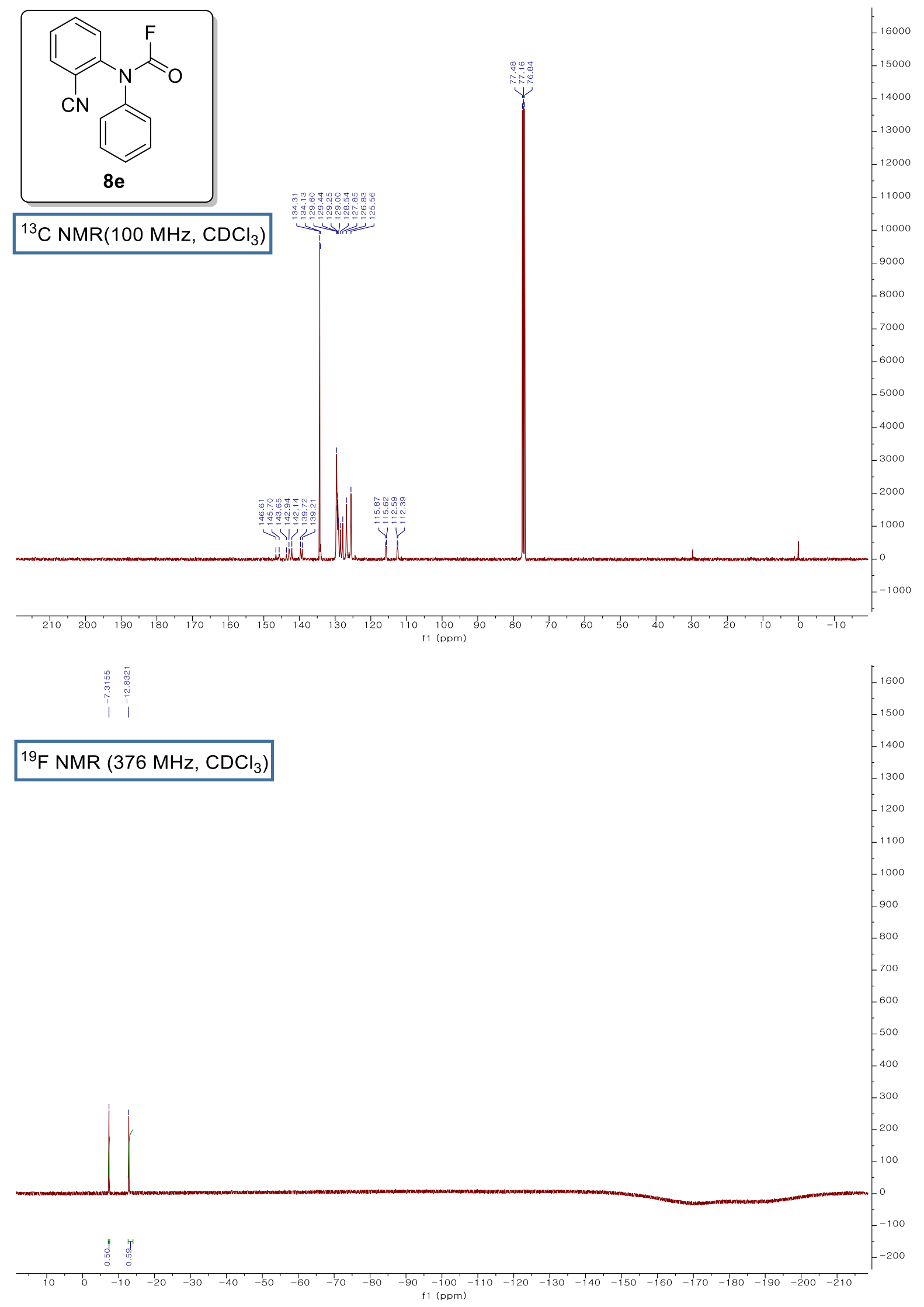




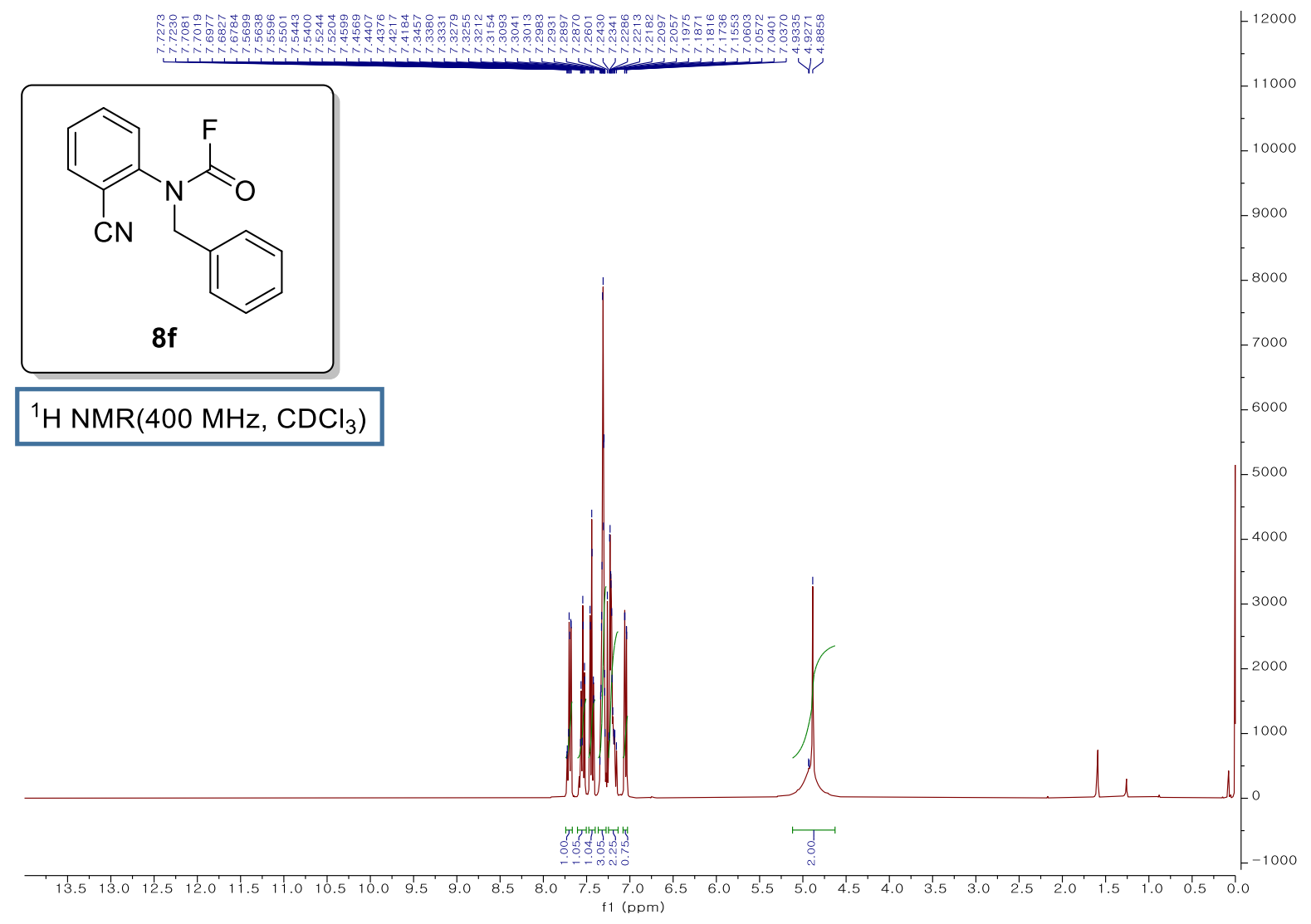

\section{${ }^{13} \mathrm{C} \mathrm{NMR}\left(100 \mathrm{MHz}, \mathrm{CDCl}_{3}\right)$}

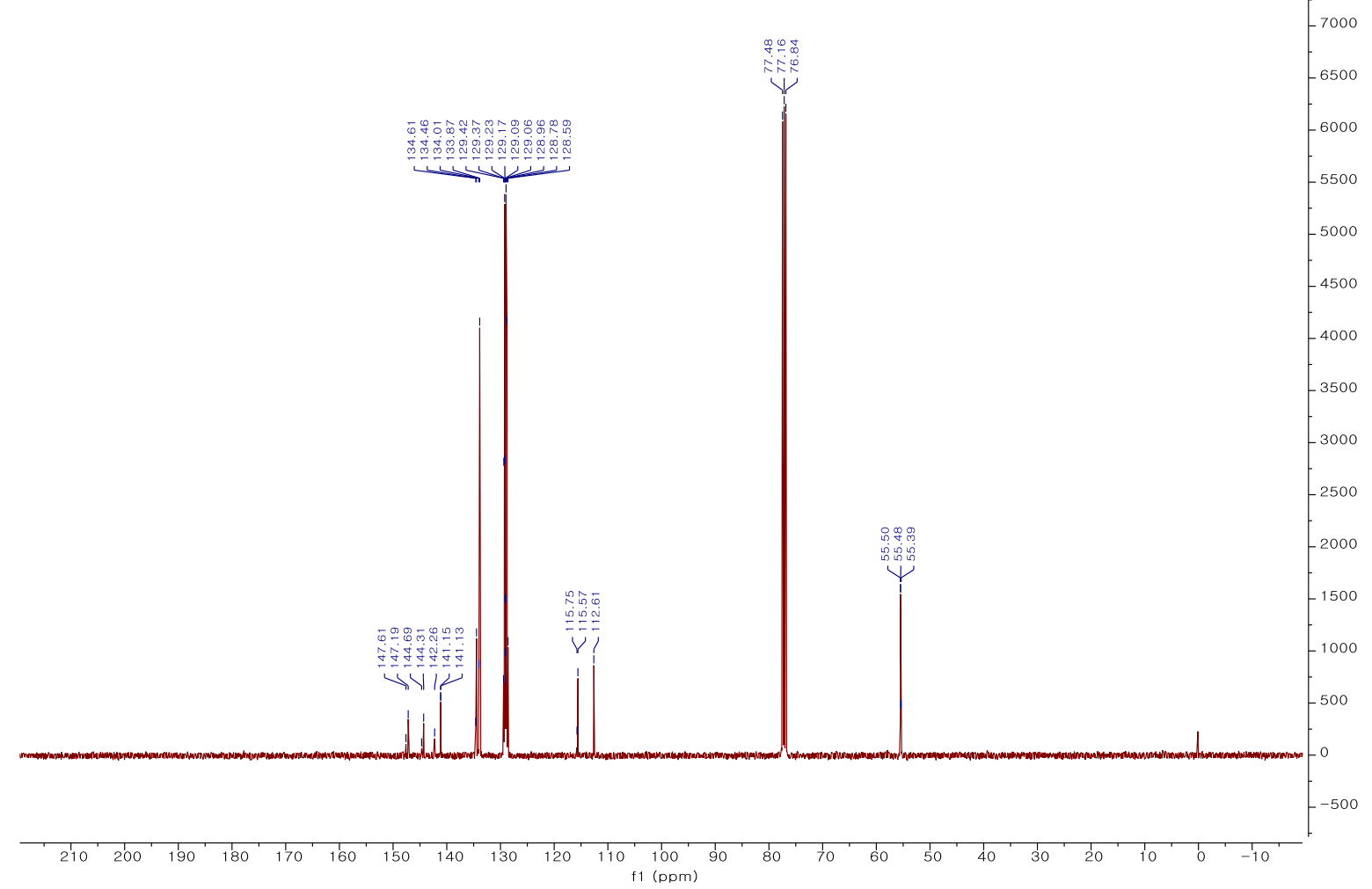




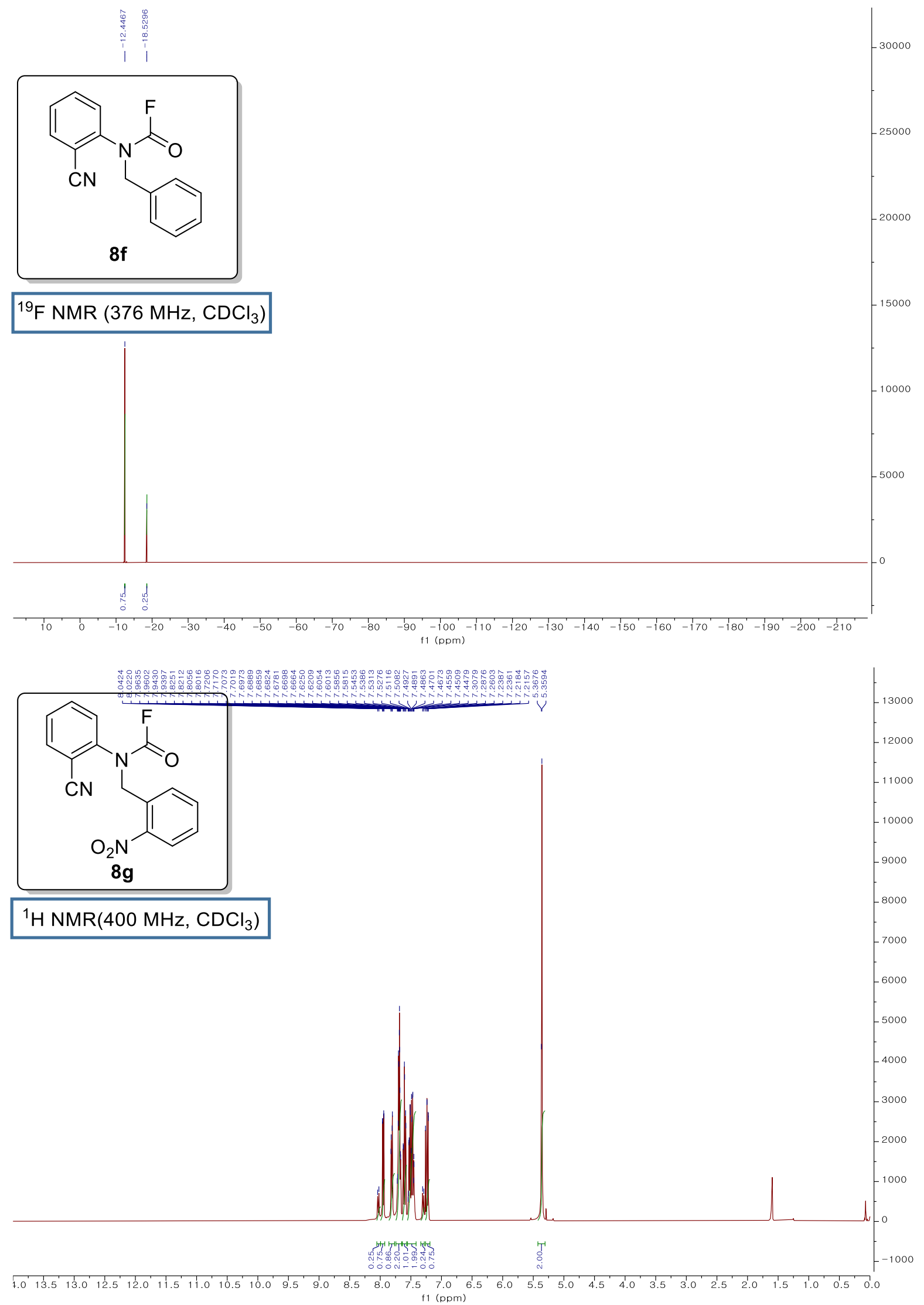




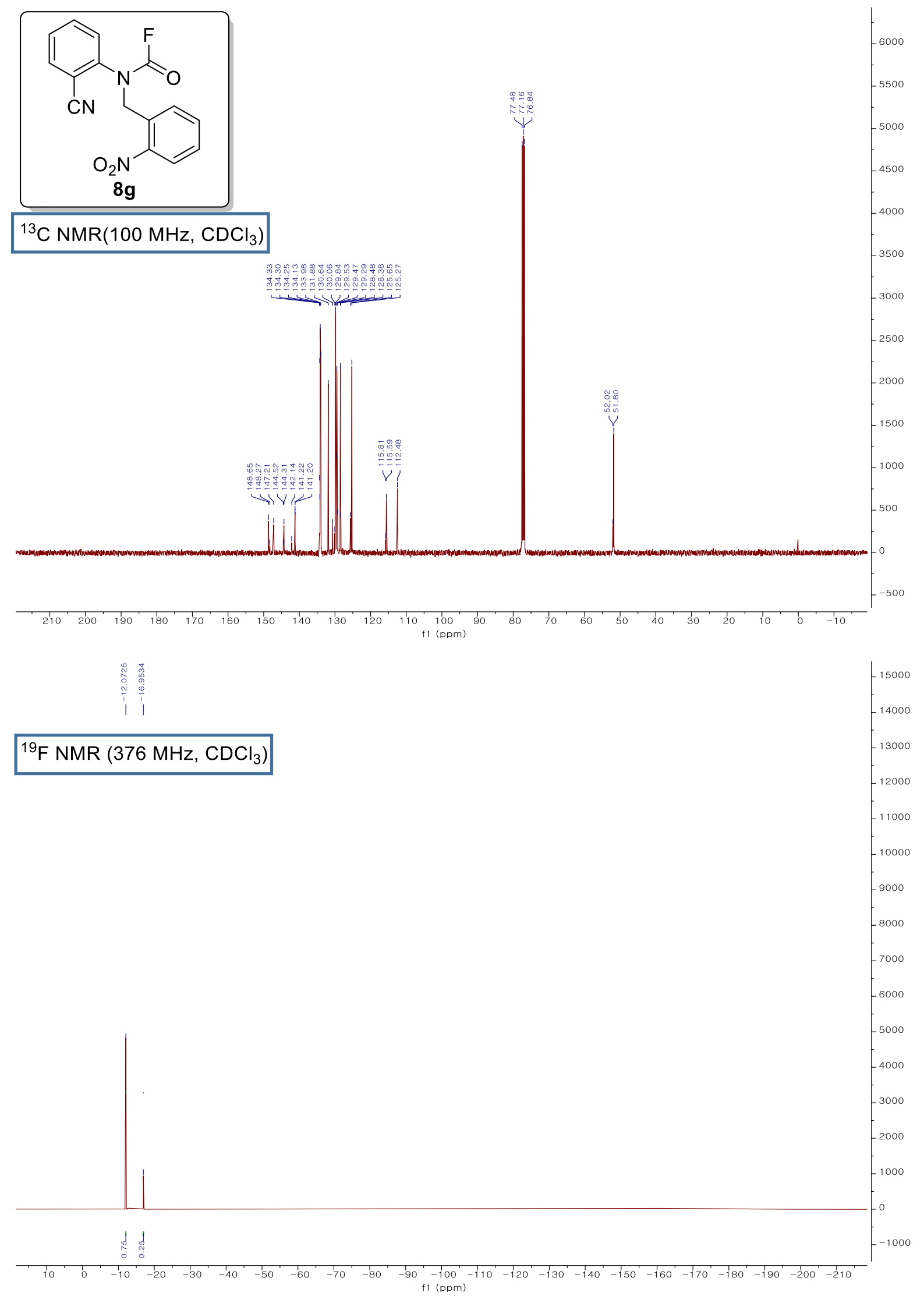



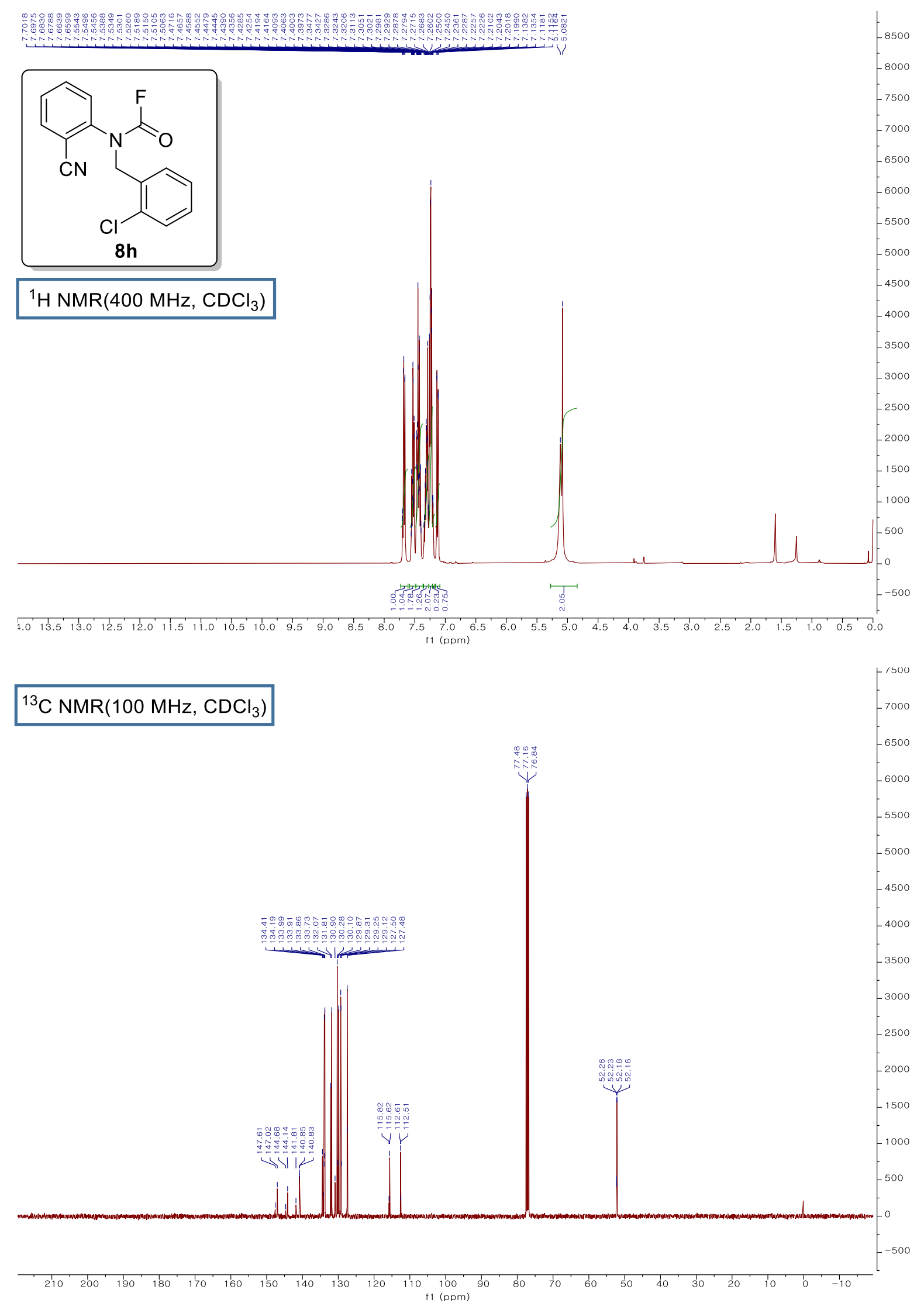


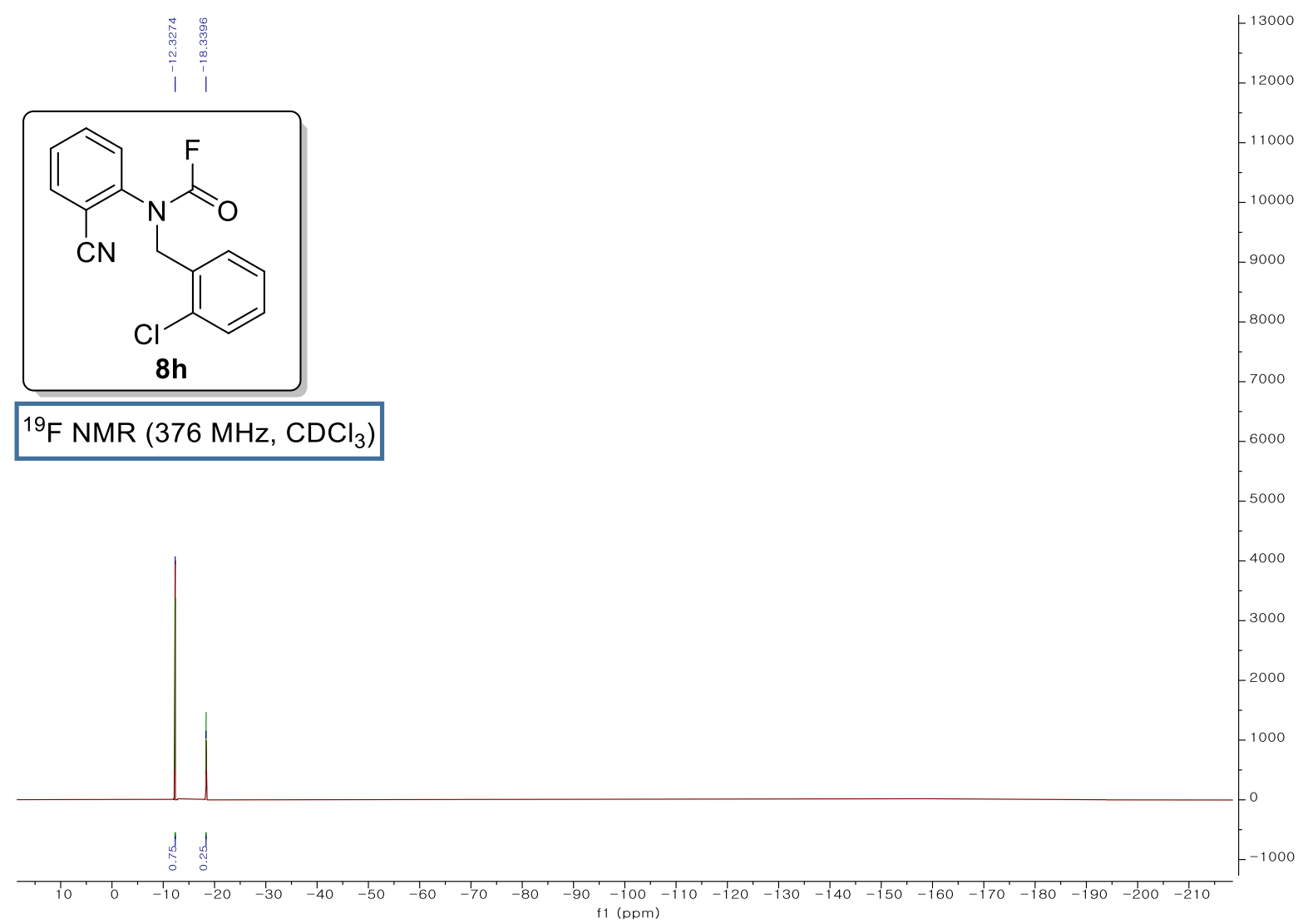

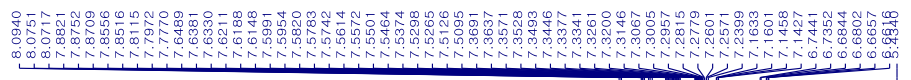

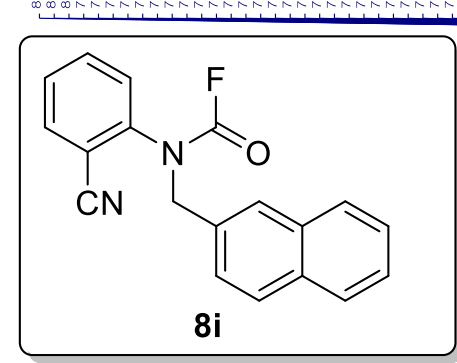

${ }^{1} \mathrm{H} \mathrm{NMR}\left(400 \mathrm{MHz}, \mathrm{CDCl}_{3}\right)$

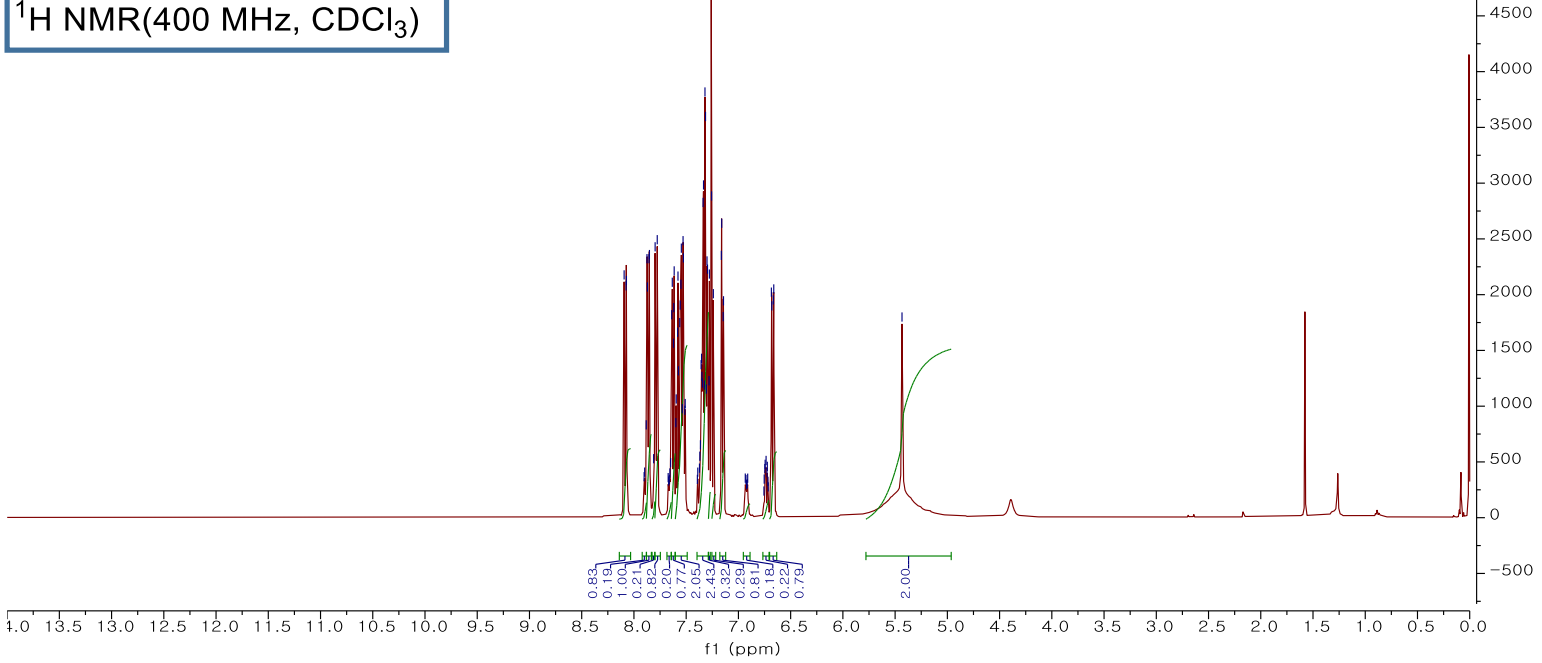



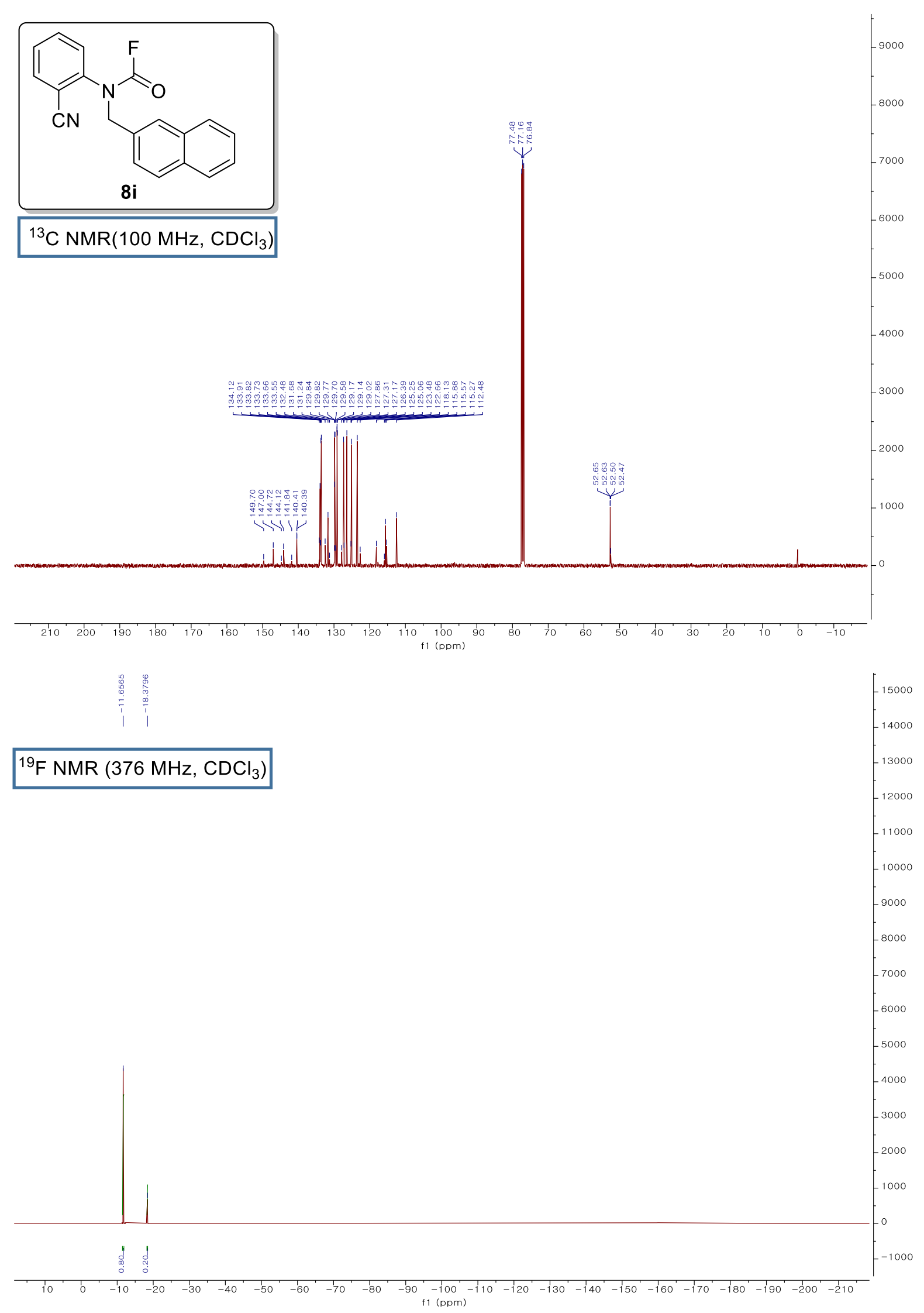

${ }^{19} \mathrm{~F} \mathrm{NMR}\left(376 \mathrm{MHz}, \mathrm{CDCl}_{3}\right)$

${ }^{13} \mathrm{C} \mathrm{NMR}\left(100 \mathrm{MHz}, \mathrm{CDCl}_{3}\right)$ 


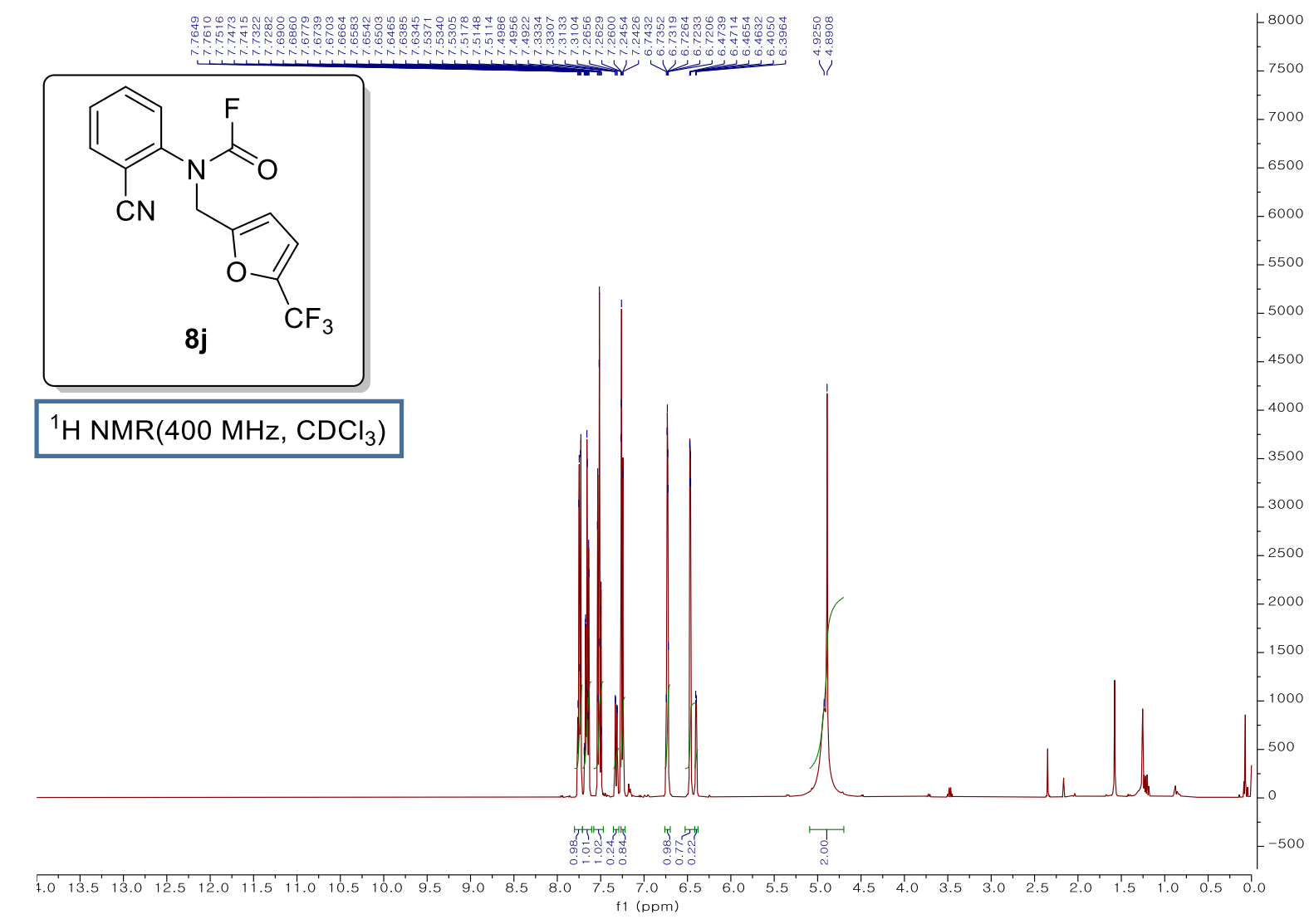

\section{${ }^{13} \mathrm{C} \mathrm{NMR}\left(100 \mathrm{MHz}, \mathrm{CDCl}_{3}\right)$}

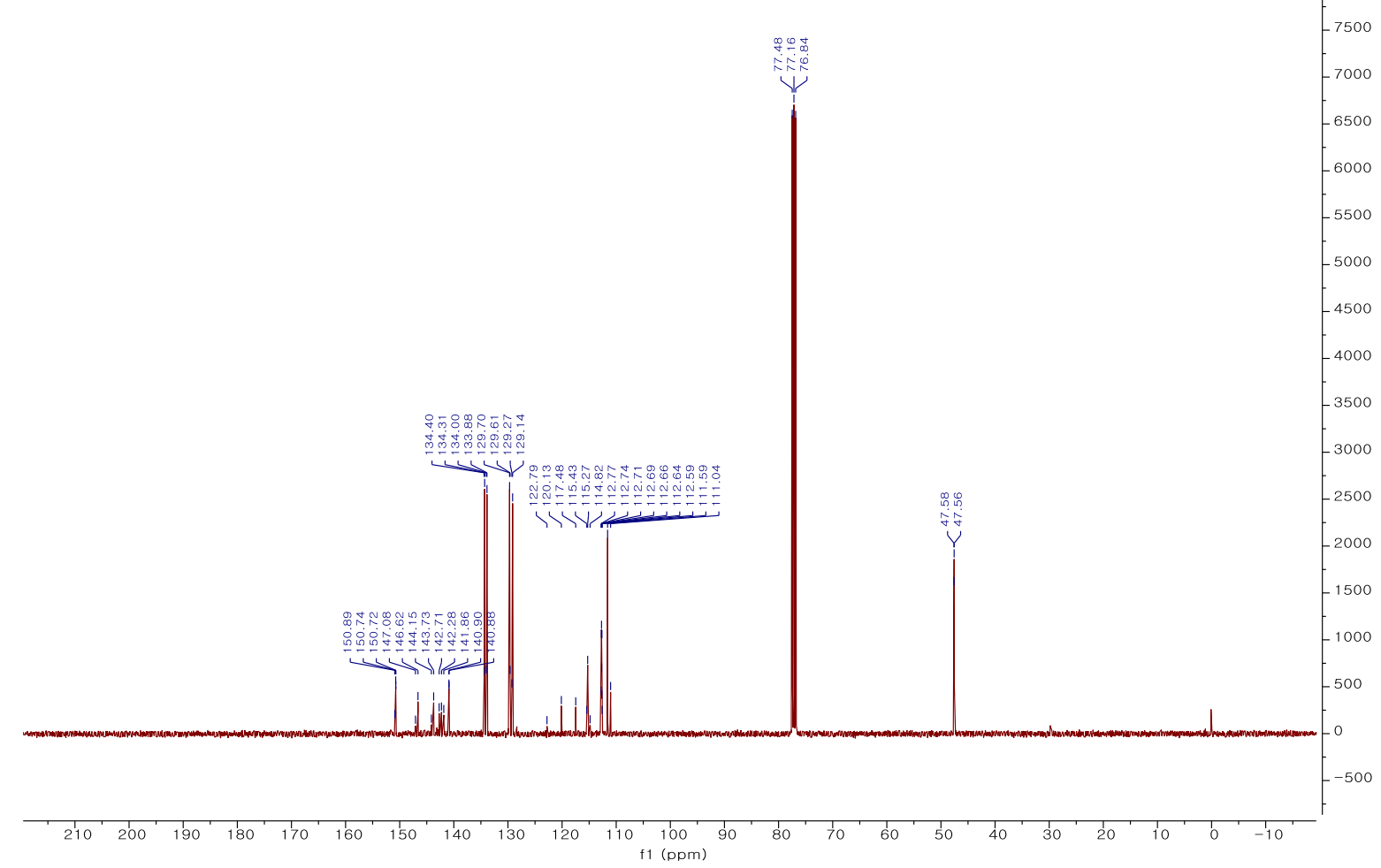




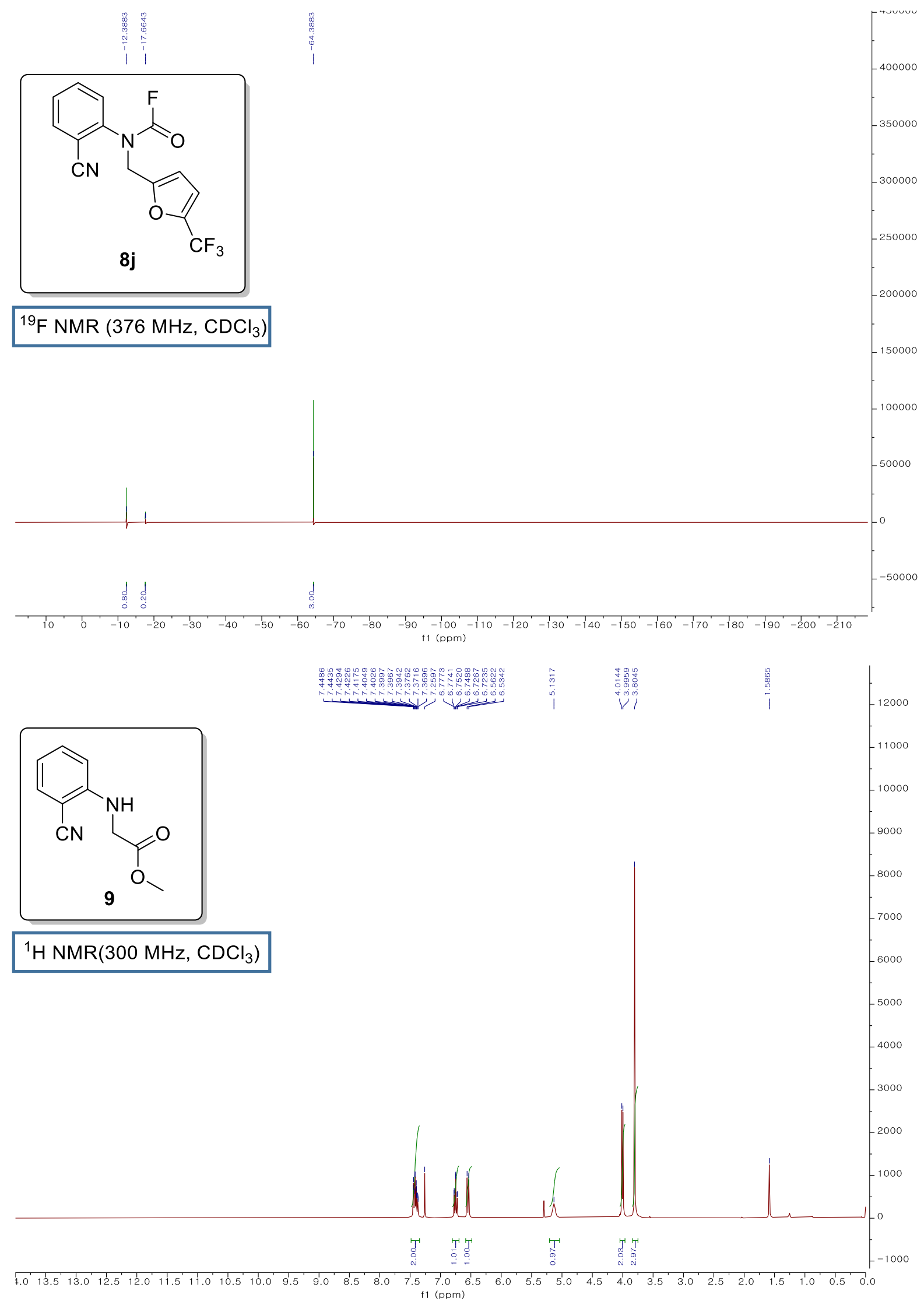




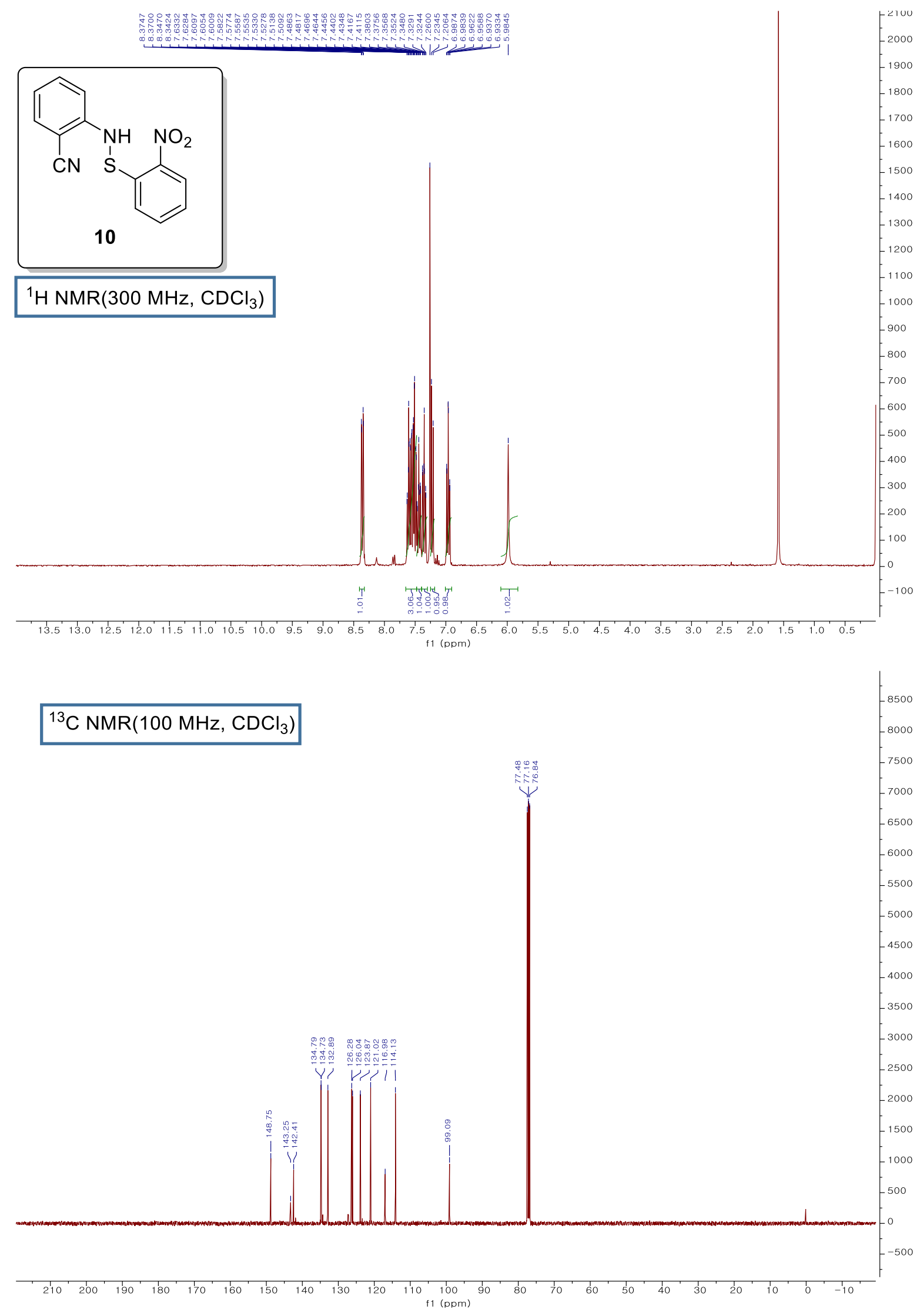




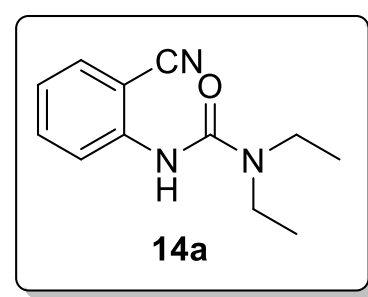

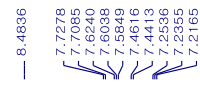
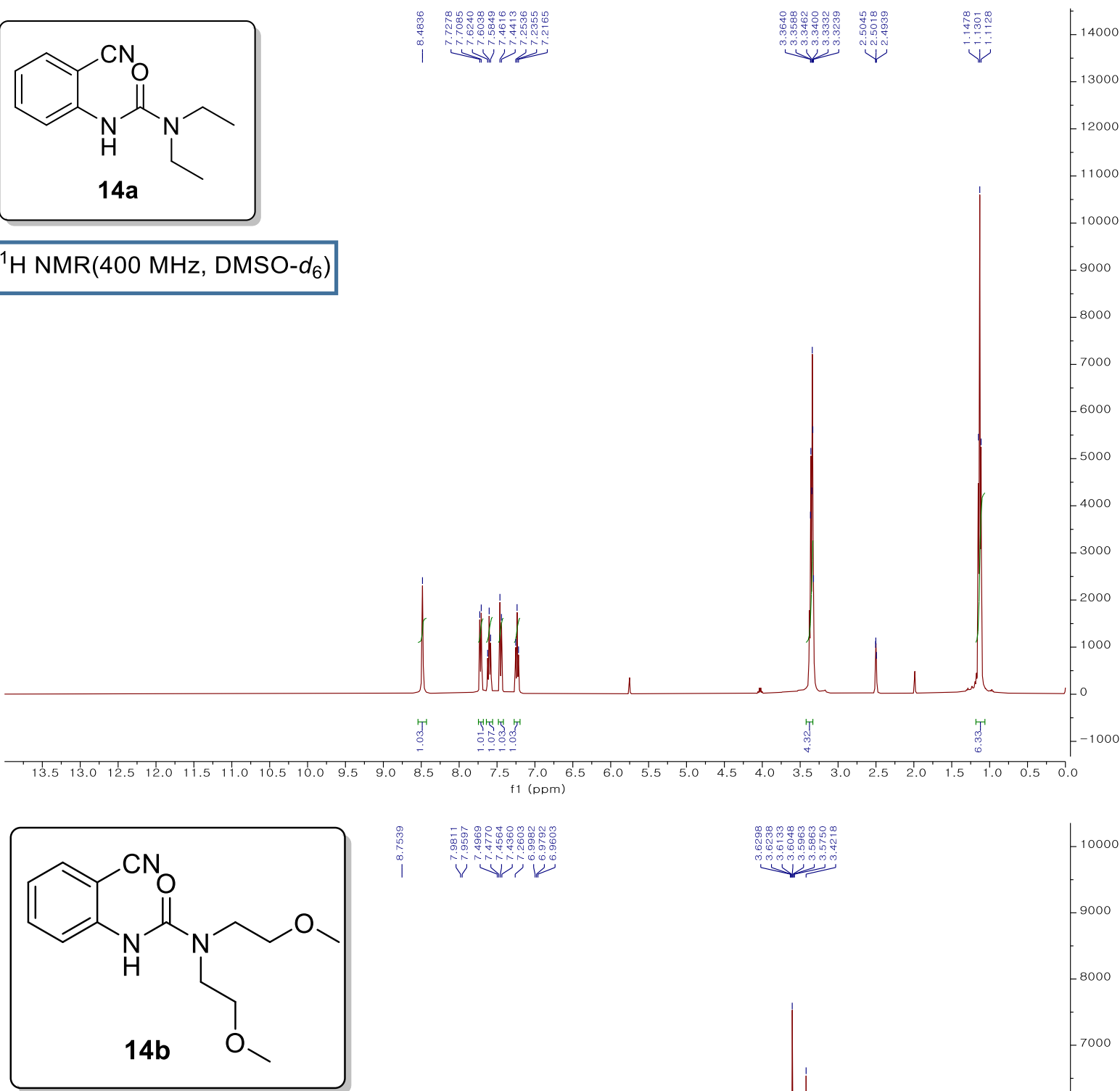

${ }^{1} \mathrm{H}$ NMR $\left(400 \mathrm{MHz}\right.$, DMSO- $\left.d_{6}\right)$

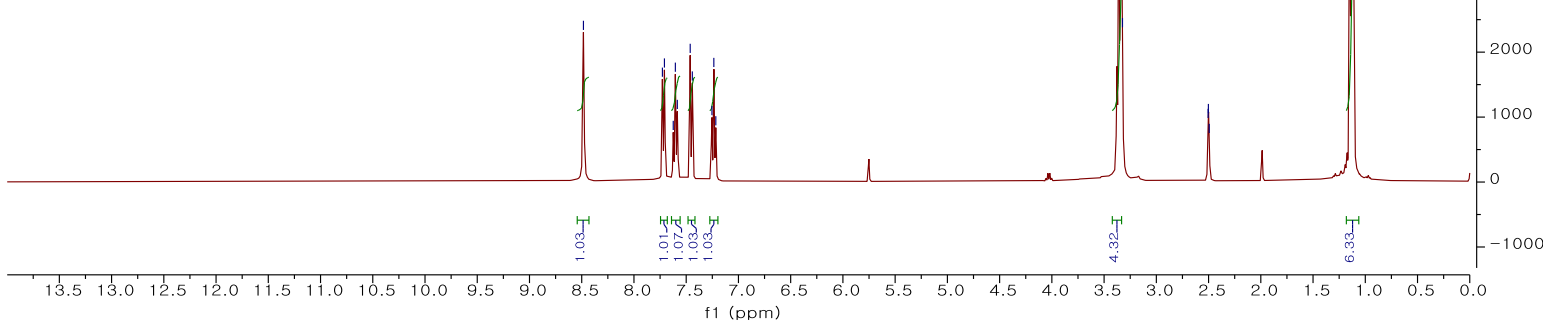

${ }^{1} \mathrm{H}$ NMR(400 MHz, DMSO- $\left.d_{6}\right)$

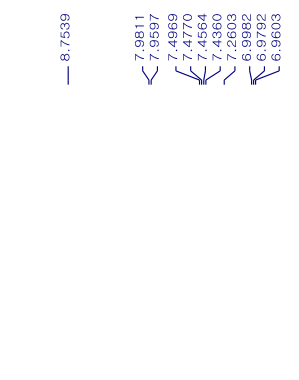




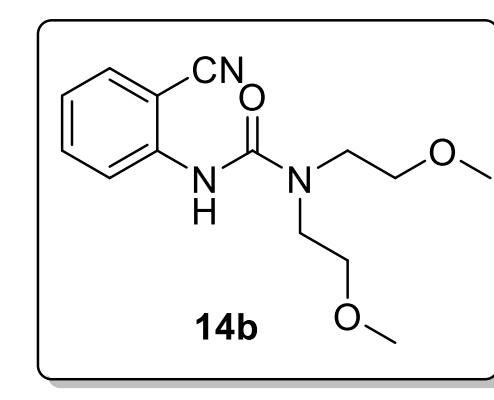

${ }^{13} \mathrm{C} \mathrm{NMR}\left(100 \mathrm{MHz}, \mathrm{CDCl}_{3}\right)$
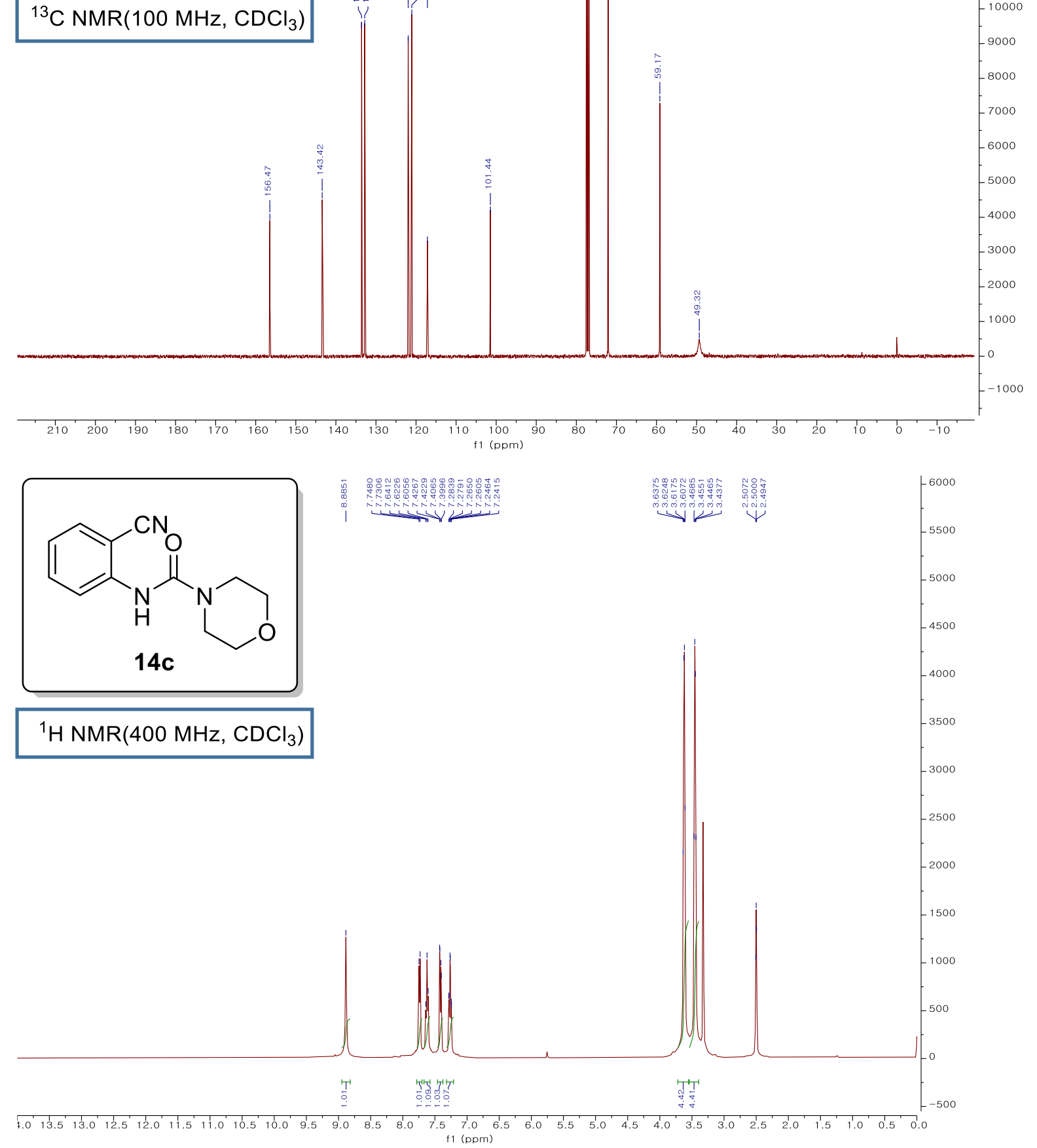

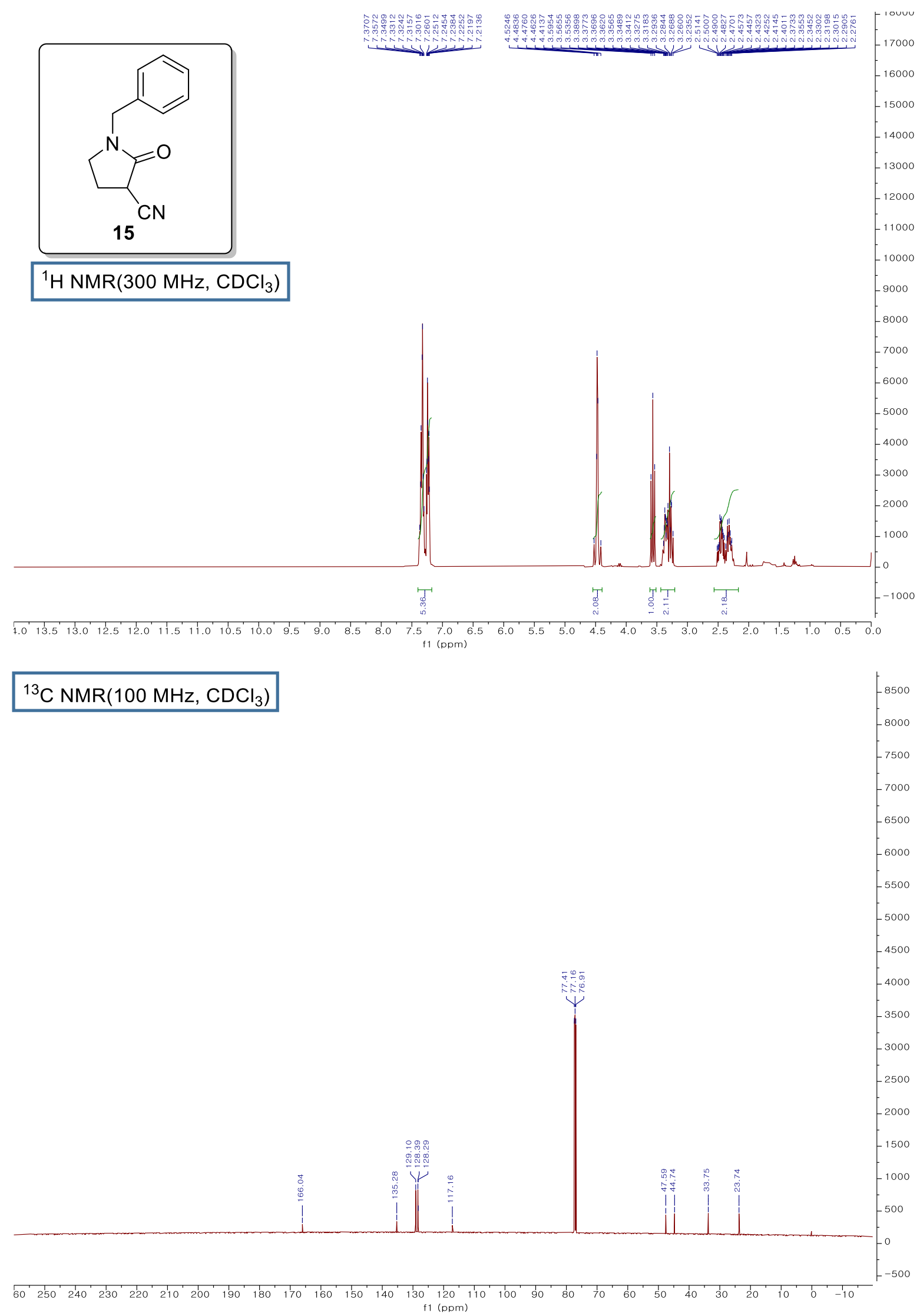

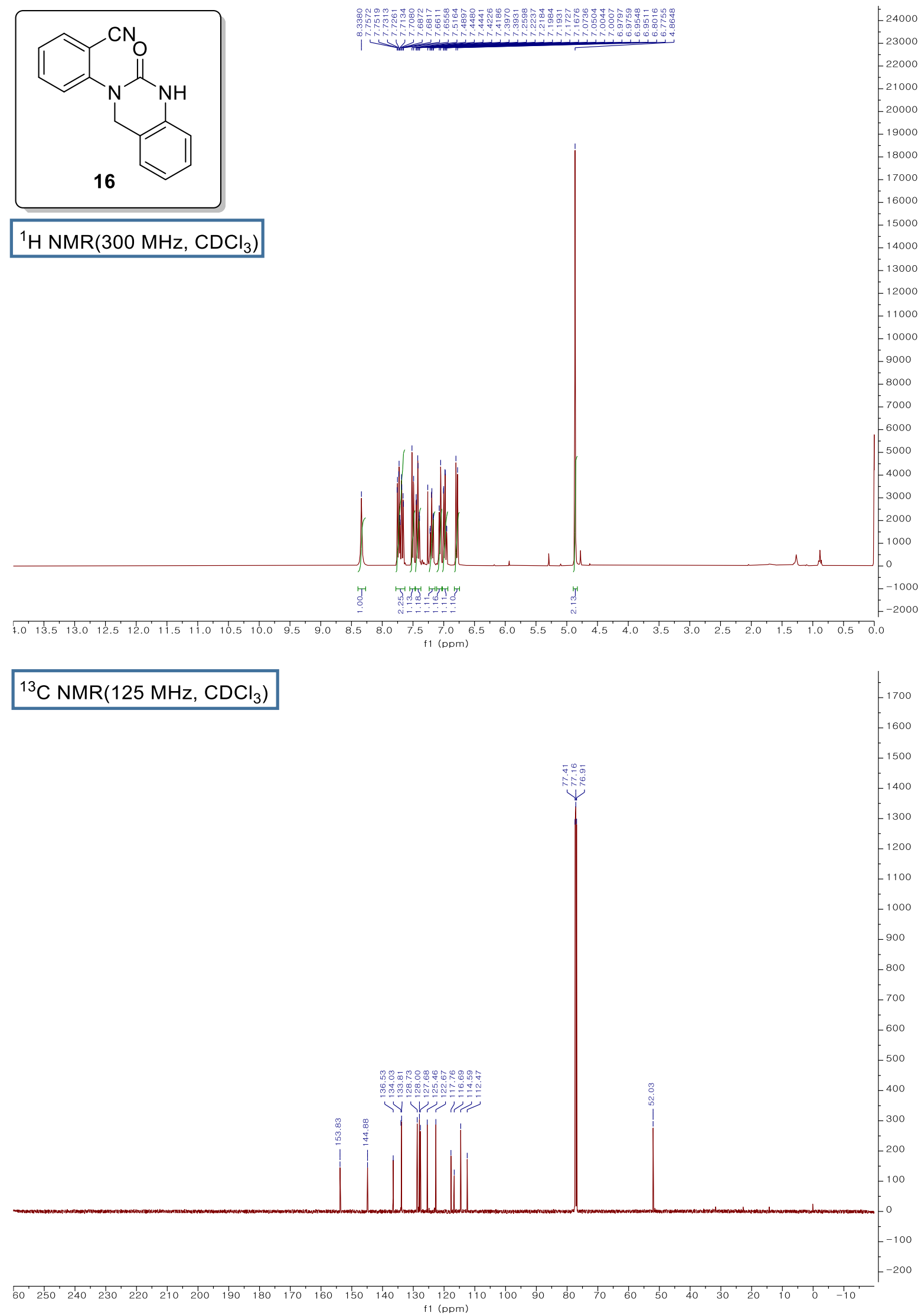